\title{
Pioneering studies on the gene order, DNA sequence and evolution of the MHC class-I region in the new world primate Callithrix jacchus
}

\author{
Dissertation \\ zur Erlangung des Doktorgrades \\ der Mathematisch-Naturwissenschaftlichen Fakultäten \\ der Georg-August-Universität zu Göttingen
}

vorgelegt von

NATALIA REGINA MESA HERRERA

aus Medellin

(Kolumbien)

Göttingen 2007 
D 7

Referent: Prof. Dr. rer. nat. Jürgen Wienands

Korreferent: Prof. Dr. rer. nat. Ernst A. Wimmer

Tag der mündlichen Prüfung: am 5. Juli 2007 
..... To my Family and Martin, who always were behind me, ..... To my Friends, who encouraged me, To my Professors, who believed in me. 
Table of contents

1. Aims and scopes....................................... 1

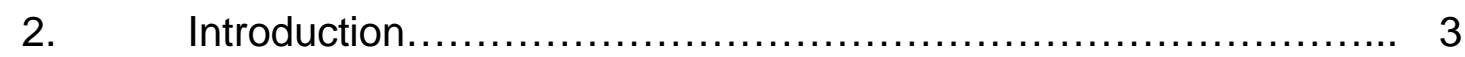

2.1 Common marmoset CALLITHRIX JACCHUS..................... 3

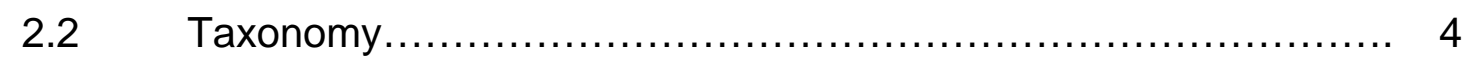

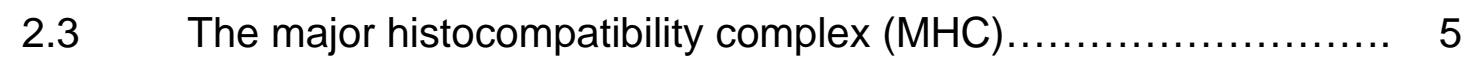

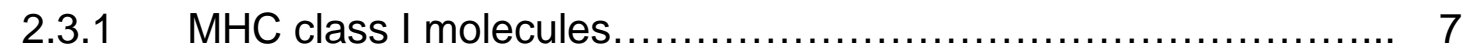

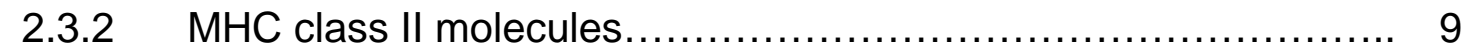

2.3.3 MHC in Callithrix jacchus and others New World Monkeys (NWM) 10

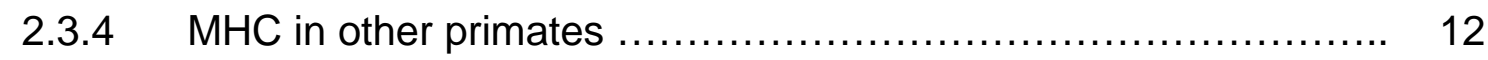

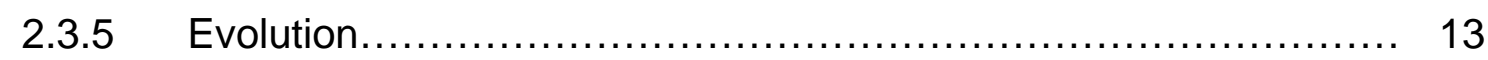

$2.4 \quad$ Framework genes hypothesis............................... 14

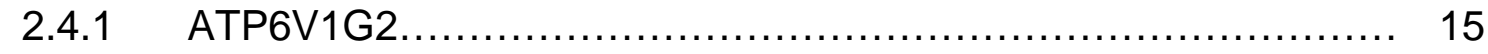

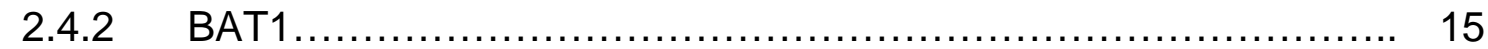

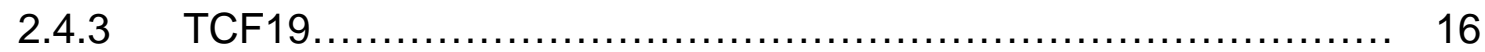

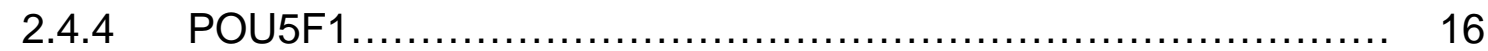

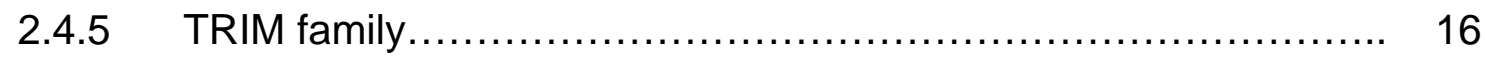

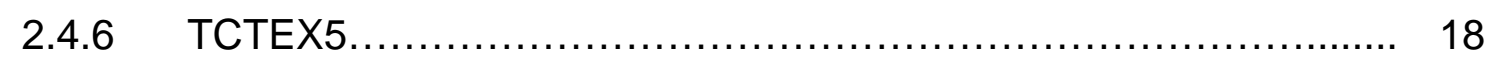

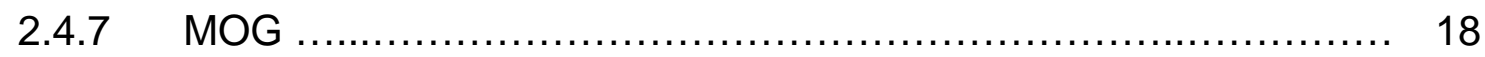

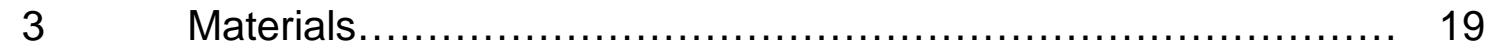

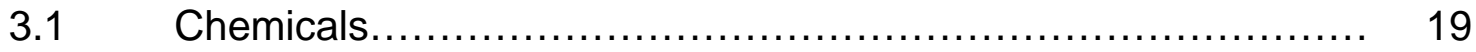

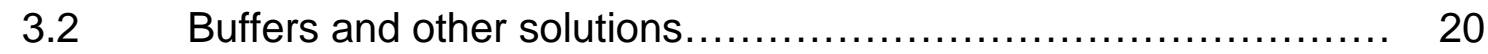

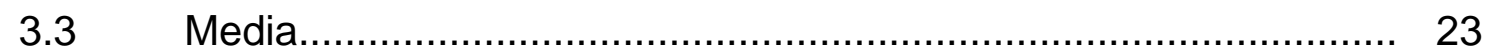

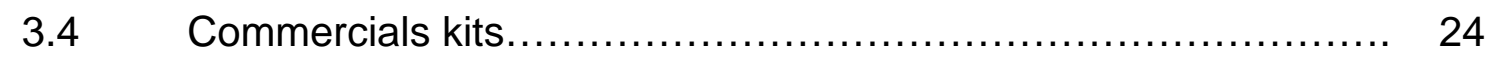

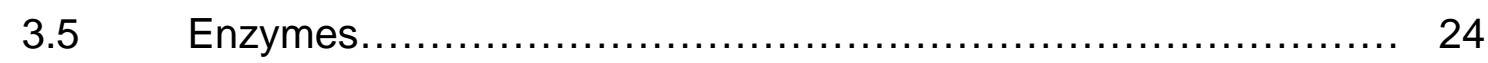

3.6 Oligonucleotides primers for PCR: sequences and annealing

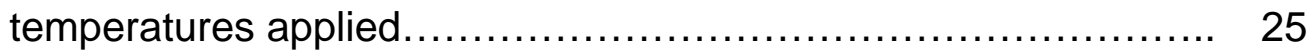

3.7 DNA ladders and other standards for comparison of molecular

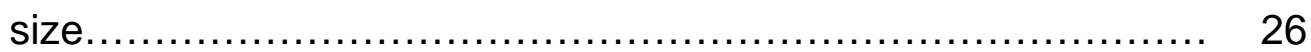

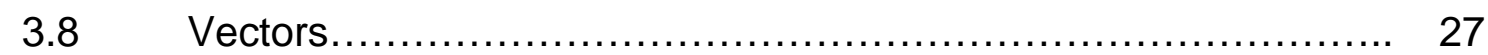

3.9 BAC-clones used for genomic analysis........................ 27

3.10 The gene sequence of Callitrix jacchus used as MHC class I probe.... 


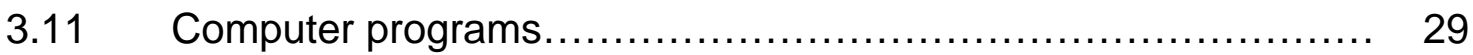

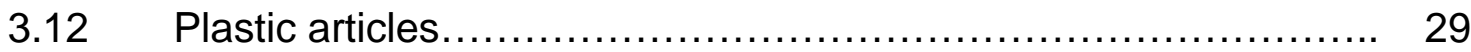

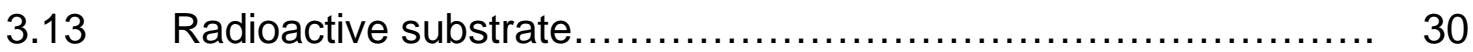

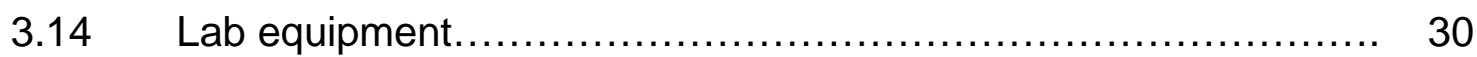

3.15 Addresses of manufacturers..................................... 32

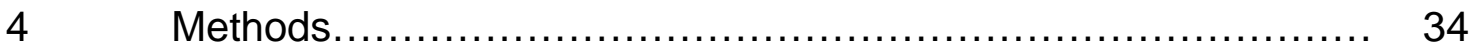

$4.1 \quad$ Preparation of materials $\ldots \ldots \ldots \ldots \ldots \ldots \ldots \ldots \ldots \ldots \ldots \ldots \ldots \ldots \ldots \ldots \ldots \ldots \ldots, \quad 34$

4.2 Filters of carrying bacterial artificial chromosome (BAC) clones for

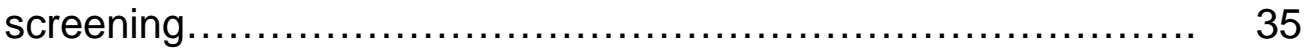

4.2.1 Source and origin of BAC bank CHORI of common marmoset (Callithrix jacchus)

4.2.2 Organization of BAC bank CHORI 259 from of the new world monkey common marmoset (Callithrix jacchus) on microtiter plates and on nylon filters............................... 35

4.2.3 Procedure for hybridization on high density filters ................ 35

4.3 Preparation of a BAC clone cultures........................... 37

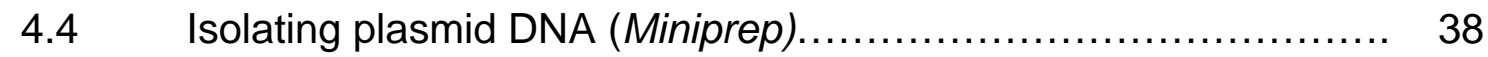

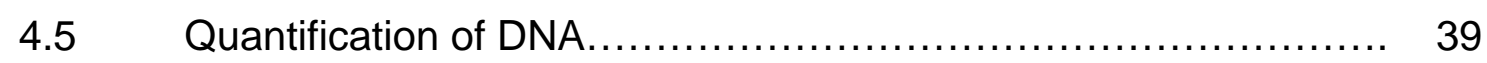

4.5.1 UV-absorption spectroscopy method......................... 39

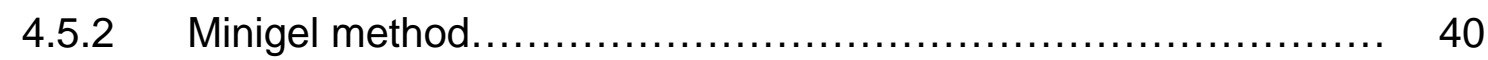

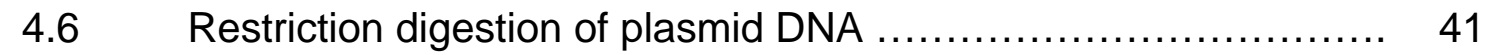

4.7 Separation of large DNA fragments by agarose gel

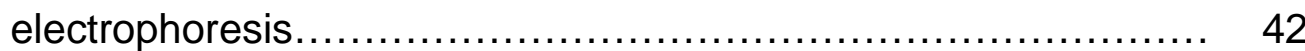

4.8 Extraction of DNA fragments from agarose gels................ 43

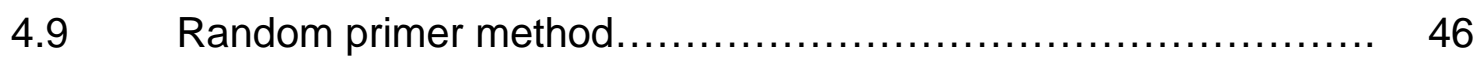

4.10 Analysis of plasmid DNA by blotting and hybridization............ 46

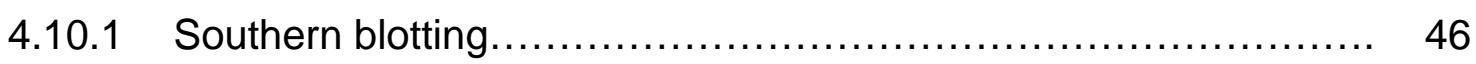

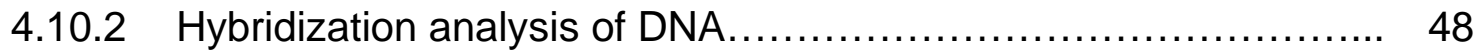

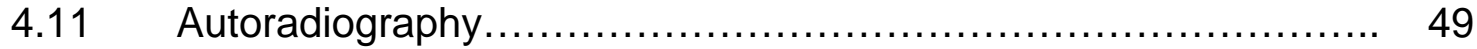

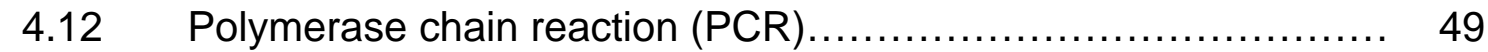

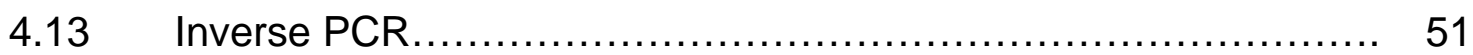

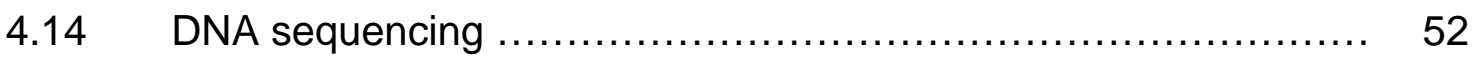

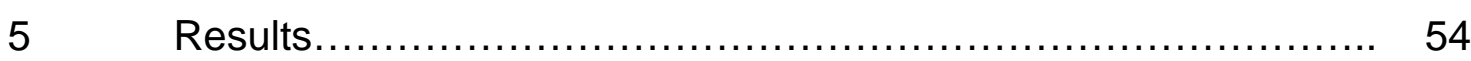


5.1 Identification of BAC clones from Callithrix jacchus containing MHC class-I region genes ................................ 55

5.1.1 Contig 1, the interval BAT1 - TCF19......................... 58

5.1.2 Contig 3, the intervals TRIM39 - TRIM26.................... 59

5.1.3 Contig 4, interval TCTEX4 - MOG............................ 61

5.2 Genomic analysis by Southern blot hybridization ................ 62

5.2.1 Contig 1, the interval BAT1 - TCF19......................... 62

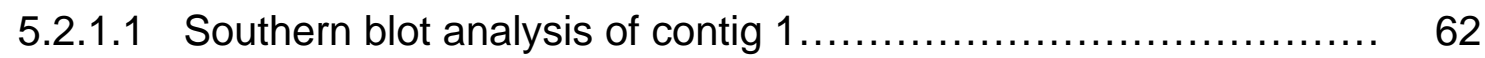

5.2.1.2 Evaluation of hybridization results (contig 1) $\ldots \ldots \ldots \ldots \ldots \ldots \ldots \ldots . \ldots 77$

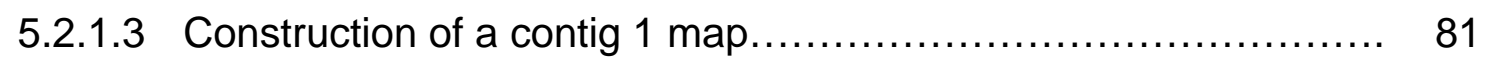

5.2.2 Contig 3, the interval TRIM26 - TRIM39....................... 84

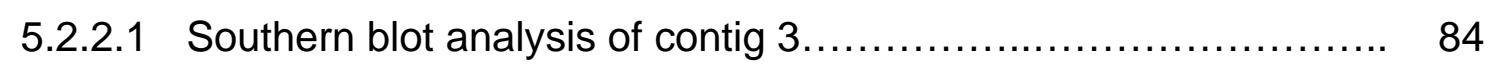

5.2.2.2 Evaluation of hybridization results (contig 3 ).................... 87

5.2.2.3 Contribution to a contig 3 map............................... 87

5.2.3 Contig 4, the intervall TCTEX4 - MOG ......................... 89

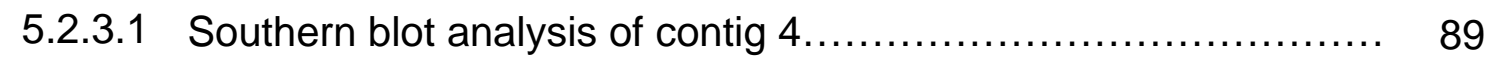

5.2.3.2 Evaluation of hybridization results (contig 4) ................... 91

5.2.3.3 Contribution to a contig 4 map.............................. 91

5.3 PCR and sequence analysis of BAC clone inserts................ 92

5.3.1 Polymerase chain reaction (PCR) analysis.................... 92

5.3.2 Approximative alignment of sequences to homologoues DNA of Hs and other mammals by BLAST .......................... 96

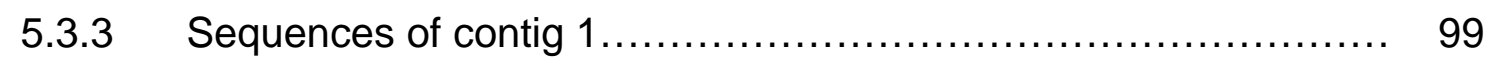

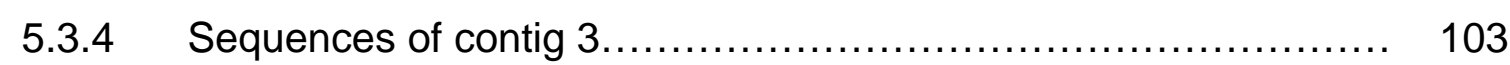

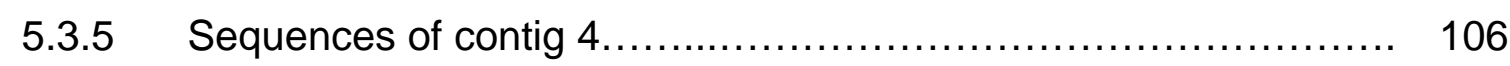

5.4 Identification of the Callithrix jacchus sequences by comparative

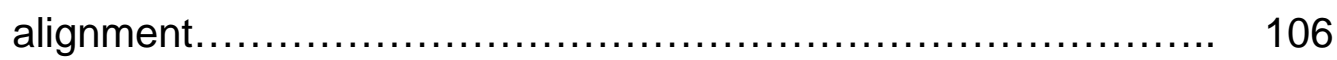

5.4.1 Programs used for identification of sequences by localization and gene assignment....................................... 106

5.4.2 Collection and presentation of sequence data................... 113

5.4.3 Demonstration of individual sequences identified by localization and possibly gene assignment in the Hs genome or proteome sequence K19161C12-TS2 
5.4.4 K28-347D1-TCF19, nucleotide sequence..................... 116

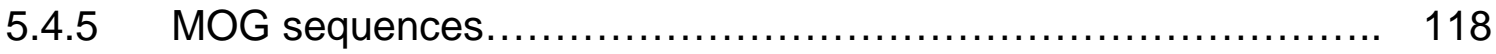

5.5 Evolution of class-I sequences and framework genes found in Callithrix jacchus compared to their homologous in other primates and mammals.................................................... 120

5.5.1 Gene trees class-I of genes............................... 120

5.5.2 Gene trees of framework genes............................. 122

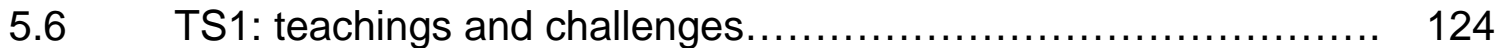

5.6.1 A gene map of TS1 and its homologous genomic vicinities in the

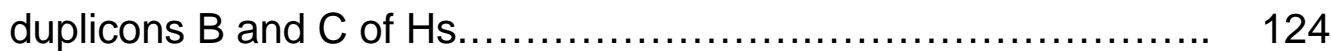

5.6.2 The two new class I pseudogenes present in the HLA-B and -C duplicons of $\mathrm{Hs}$, Patr and other mammals, also in the 14 Mamu-B duplicons.

5.6.3 A MHC ferritin heavy chain pseudogene in Callithrix jacchus....... 134

5.6.4 Characterization and possible interpretations of "Vorspann" v, the sequence next to which a ferritin transposon has been inserted....

6 Discussion....

6.1 Physical map of the intervals from MHC class-I................. 144

6.1.1 Physical map of the BAT1 - TCF19 interval (contig 1)............ 144

6.1.2 Physical map of the intervals CAT56 - TRIM39 and TRIM 39 TRIM26 (contig 2 and contig 3 respectively)................... 145

6.1.3 Physical map of interval TCTEX4 and MOG (contig 4)........... 145

$6.2 \quad$ Sequence analysis of BAC clones............................ 146

6.2.1 BAT1 gene in Callithrix jacchus............................. 146

6.2.2 TCF19 gene in Callithrix jacchus............................ 146

6.2.3 MOG gene in Callithrix jacchus............................. 146

6.3 On alignment of sequences in general and in particular those of the Callithrix jacchus....................................... 147

6.3 On pseudogenes in the MHC class I region..................... 147

$6.4 \quad$ On duplicons in the MHC class I region......................... 148

6.5 On transposon especially the pseudo ferritin found in MHC Caja

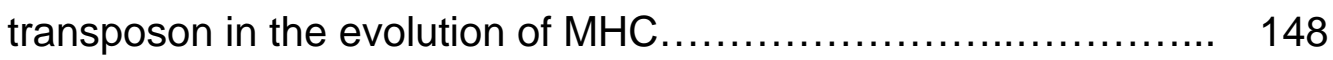

6.6 On potential value of sequence TS1 for molecular immunogenetics................................................ 148 


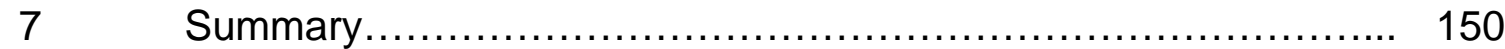

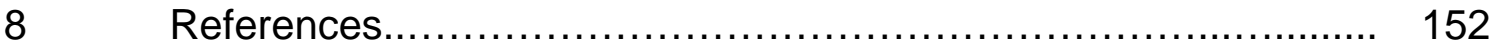

$9 \quad$ Annex: The 55 Callithrix jacchus sequences..................... 175

Acknowledgements

Lebenslauf 
If you cannot study function, study structure!

Francis Crick

\section{Aims and scopes}

The object of these pilot studies is to contribute to the structural analysis of the MHC class I region in a lower primate, the new world monkey Callithrix jacchus (Caja).

The aims are:

- Selection of BAC clones containing inserts apt to genomic and sequence analysis of the $\mathrm{MHC}$ class I region;

- Establishing the contigs belonging MHC class I in Callithrix jacchus of inserts for obtaining a restriction map and hybridization with class I probe and framework gene, this framework gene is flanking the MHC class I region.

- Typifying BAC clone inserts selecting various primers by PCR. This knowledge should be useful for a complete walking chromosome sequencing of the entire class I region in Caja having been started recently by Prof. T. Shiina;

- Identification and localization of the 55 sequences obtained by alignment in the Hs genome and other species.

- Using the special opportunity of studying one sequence, TS1 containing apparently three different kinds of pseudogenes, interesting by an arrangement on both DNA strands, much more however, as they seem to concern three unsolved basic problems in immunogenetics, i.e. gene clustering by duplication, control of gene expression by promotor regions (5'FR), and transposition of (pseudo)genes.

The scope of these pilot investigations in some parts is methodology, like

- testing an unusual way of concluding a gene order,

- analyzing partial information about MHC class I region in Caja,

- supporting it by localization and assignment of 55 Caja sequences obtained.

Basically, intentionally and successfully, however, the scope of these studies is the ory. This becomes obvious in studies of, and suggested by the Caja sequence K161C12-TS1:

(1) By its two class I pseudogenes, it seems to offer a useful key for the analysis of the known gene clustering by duplication, may be even of the descendence and 
evolution of their neighbors, the HLA genes B, C and others in Hs and Patr - likewise the 14 B genes in Macaca mulatta

(2) Second, a principle for the insertion of transposons might be exemplified structurally by the rather young ferritine pseudogene apparently unique to Caja being (3) inserted next to a sequence $v$ that by comparison with other 5'FR may be suspected of being an ancient promotor region. Strangely enough, read in the opposite direction, on its complementary strand, it turns out to be highly identical and almost completely coincident with the Hs class I pseudogene. 


\section{Introduction}

\subsection{Common marmoset Callithrix jacchus}

The word "marmoset" is an old French word for "grotesque figure". The South American primate genus Callithrix comprises two groups of species, namely, the subgenera of jacchus and argentata. Five taxa have been proposed for the jacchus group: C.j. jacchus, C.j. aurita C.j., penicillata, C.j.kuhlii, and C.j. geoffroyi; these, however, at present still under discussion (Nagamachi et al., 1997). The jacchus group lives in the Atlantic forest of eastern Brazil, in the states of Maranhão and Ceará northeast of São Paulo, see Figure 2.1 (Nagamachi et al., 1997). The animals are inhabitants of the canopy of secondary rain forests and gallery forests.

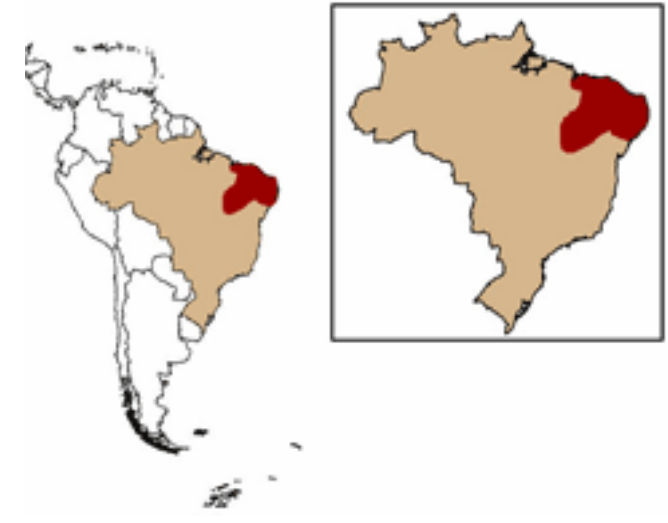

Figure 2.1: Area of distribution of Callithrix jacchus in the Atlantic forest of eastern Brazil, the states of Maranhão and Ceará northeast of São Paulo (http://pin.primate.wisc.edu/factsheets/entry/common_marmoset/taxon)

They are the smallest higher primates, characterized by a small body of about $50 \mathrm{~cm}$ length head to tail and $350-450 \mathrm{~g}$ average weight (Warren et al., 1997). This species is easily identified by the (two) white tufts of hair on both sides of the head, and a white blaze on the forehead. The body is covered with soft thin hair, grayish in color with darker stripes. The tail has darker-colored rings. Females are a little larger than males. Common marmosets have large chisel-like incisors used for gnawing holes in trees, the canines are shaped more like incisors (Groves, 2001). The dental formula of common marmoset is 2:1:3:2 on both the upper and lower jaw (Martin, 1990). The labium major of the female resembles the scrotum of the male. There is little sexual dimorphism in this species. The common marmoset has no opposable thumbs. It is omnivorous, feeding on a variety of food-stuffs, including fruits, insects, 
snails, and small vertebrates such as lizards. To eat gum it gouges holes in tree trunks with its large incisors and laps the exudates from the hole.

(www.damisela.com/zoo/mam/primates/callitrichidae/jacchus/taxa.html)

Its main predators are hawks and small carnivorous mammals such as the tayra (Stevenson and Rylands, 1998) (http://www.saudeanimal.com.br/sagui.html).

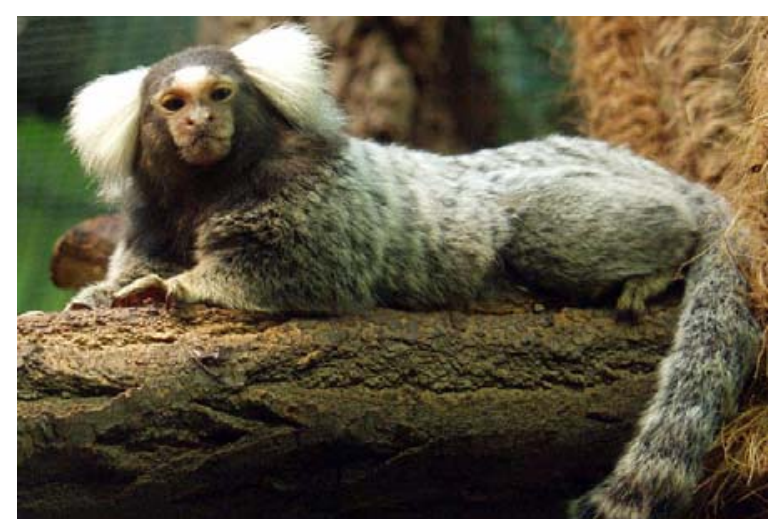

Figure 2: Marmoset (Callithrix jacchus).

(Photo courtesy of Raimond Spekking, Wikimedia Commons, www.genome.gov.).

Callithrix jacchus monkeys begin their activities early in the morning until the dusk; they live on the branches of the trees using their claws like adapted nails. Their territory is marked by the scents that unfold from the glands of the chest, abdomen, the genitals and urine, these marks allow among them the identification of the species, relationship, and receptivity sex. The groups generally include 8 to 12 animals and their social structure is very varied. The mating pairs are monogamous. The time of gestation is 140 to 150 days, and of one to three individuals are born (http://www.univie.ac.at/zoologie/theo/marmoset/calli.html)

\subsection{Taxonomy}

Common Name: white-tufted-ear marmoset [English]

Kingdom: Animalia

Subkingdom: Eumetazoa

Branch: Bilateria

Phylium: Chordata

Sub-phylium: Vertebrata

Superclass: Gnathostomata 


\author{
Class: Mamalia \\ Subclass: Eutheria \\ Order: Primates \\ Suborder: Haplorrhini \\ Infra-order: Simiiformes \\ Section: Platyrrhini \\ Family: Callitrichidae \\ Subfamily: Callitrichinae \\ Genus: Callithrix
}

Species: Callithrix jacchus

(http://www.damisela.com/zoo/mam/primates/callitrichidae/jacchus/taxa.html)

(http://www.itis.usda.gov/servlet/SingleRpt/SingleRpt?search topic=TSN\&search val $\underline{\text { ue }=572915)}$

\title{
2.3 The major histocompatibility complex (MHC)
}

$\mathrm{MHC}$ is a group of polymorphic genes or multigene family of immune system genes that code cell surface glycoproteins which are found on the surface of cells which present peptides to circulating $T$ cells and which help the immune system to recognize foreign substances (Go et al., 2003). In 1940, the geneticist George D. Snell performed experiments of grafts of skin in mice, demonstrating that an immune reaction of the animal against the grafted weave cause the rejection (Snell, 1948; lein, 2001). The term histocompatibility comes from the Greek word histos = tissue and the word "compatibility" in order to talk about the molecules with a function in the reaction of transplants although this is not its true physiological role (Snell, 1948; Snell, 1951).

MHC proteins are found in all higher vertebrates, from fish and birds to non-human primates up to the human. They have been shown to display the levels of genetic polymorphism and further the molecular evolutionary processes which generate the diversity required to the immunity. The complex is called in the human the human leukocyte antigen (HLA) system, for non-human species LA is used the prefixed species name, e.g. BoLA (The MHC Sequencing Consortium, 1999).

In the human, the MHC spans almost four megabases (4.000.000 bp) on the short arm of chromosome 6 , band p21.3, it includes approximately 224 coding and noncoding sequences, of which about half have known immunological functions. It also 
includes 96 pseudogenes, non-functional gene remnants (Kulski et al., 2005; Beck et al., 2000; Go et al., 2003; The MHC Sequencing Consortium, 1999).

The MHC has been associated with a role in influencing propensity for known autoimmune diseases such as insulin-dependent diabetes mellitus, multiple sclerosis, systemic lupus erythematosus, myasthenia gravis, and rheumatoid arthritis (Wong et al., 2005; Bach, 2005; Lie, 2005; Reveille, 2000; Azizah et al., 2004). The MHC contains genes contributing to several other hereditary disorders that are either not autoimmune in nature or in which the role of autoimmunity is uncertain. These include ankylosing spondylo arthropathies, where there is a clear association with the class I allele HLA-B27, and narcolepsy, equally clearly associated with certain class II alleles (Luthra-Guptasarma and Singh, 2004). The MHC also includes the genes for steroid 21-hydroxylase and hemochromatosis (Nardi et al., 2003). Hemochromatosis is one of the most common simple Mendelian disorders of man, it has been found a 250-kilobase region more than three megabases telomeric of the major histocompatibility complex $(\mathrm{MHC})$. It has been identified related with HLA-H containing a two missense alterations (Feder et al., 1999; Weitkamp et al., 1994). Recently, two major quantitative trait loci for dyslexia have been mapped distal to the MHC (Stein, 2001) and also for Parkinson's disease (Eslamboli, 2005).

The MHC is divided into three subgroups called MHC class I, MHC class II and MHC class III regions, each containing groups of genes with related functions. The MHC has shown conservation of paralogous genes in the class I, II and III regions, but differences in position, type and number of immunoglobulin-related genes (Amadou et al., 1999; The MHC sequencing consortium, 1999; Daza-Vamenta et al., 2004; Hurt et al., 2004; Anzai et al., 2003; Neff, 2005). The MHC, particularly in the human, displays strong allelic diversity therein, especially among the nine classical HLA genes. The most conspicuously diverse loci are present in human HLA-A, HLA-B, and HLA-DRB1, with roughly 472, 805, and 256 known alleles, respectively (http://www.ebi.ac.uk/imgt/hla/stats.html).

The molecular genetic structure of the MHC is considered to be an evolutionary result of selective pressure imposed by infectious microorganisms (Ploegh, 1998). Several studies have compared the genomic organization of the MHC in (other) organisms, as mouse, chicken, rat, cat, zebra fish and Fugu fish (Trowsdale, 1995; Timon et al., 1998; Graser et al., 1999; Yuhki et al., 2003) as well as in more primitive members of the chordates. 
The way of "inherited in block" is used by the MHC producing haplotypes, although with rare exception there are events of recombination (Trowsdale, 1995).

A haplotypical association is usually stronger and more meaningful than an allelic association. The co-dominant expression and haplotypical transmission have an important consequence: within a family, HLA-identical sibling frequency should be 25\% according to Mendelian expectations (Alper et al., 1992; Degli-Esposti et al., 1992; Gaudieri; et al., 1997).

This means that certain alleles tend to occur together in the same haplotype rather than randomly segregating together. This is called linkage disequilibrium (LD) and is quantitated by a $\Delta$ value (Mattiuz et al., 1971 and Begovich et al., 1992).

\subsubsection{MHC class I molecules}

The MHC class I encodes heterodimeric peptide-binding glycoproteins as well as antigen-processing molecules such as TAP and tapasin (Leonhardt et al., 2005; Herberg et al., 1998; Pamer and Cresswell, 1998). The molecules contain two noncovalently linked polypeptide chains, i.e., one MHC class I encoded heavy chain, called the $\propto$ chain of 44 to $47 \mathrm{kD}$ with the domains $(\propto 1, \propto 2$ and $\propto 3)$ and one nonMHC encoded subunit, a $\beta_{2}$-microglobulin of $12 \mathrm{kD}$. The molecular complex has four domains, three formed from the MHC-encoded $\propto$ chain, and one of the $\beta_{2}$ microglobulin (Jones et al., 1988).

Three quarters of the total polypeptide - the $\propto$ chain - form an extracellular chain, a short segment is the trans-membrane, the carboxy terminal end extends into the cytoplasm, see Figure 3.

The peptide-binding site has a size of approximately $25 \AA \times 10 \AA \times 11 \AA$. It is formed by two amino terminal segments $\propto 1$ and $\propto 2$ of the $\propto$ chain, both approximately 90 residues long. These interact to form an eight-stranded, antiparallel $\beta$-pleated sheet, the bottom supporting the two parallel $\propto$-helical strands. The variations among different class I alleles, important for peptide binding and $\mathrm{T}$ cell recognition, are contributed by the polymorphic or variable amino acid residues of class I proteins confined to the $\propto 1$ and $\propto 2$ domains. Peptides that bind to the groove of an MHC class I molecule are usually $8-10$ amino acid residues long, and interact with their back bone by a series of hydrogen bonds and ionic interactions at both ends. A cluster of tyrosine residues, common to all MHC class I molecules, form hydrogen bonds to the amino terminus of the peptide bound, while binding to the carboxy end 
terminus of the peptide back bone in addition to the carboxy terminus itself (Flutter and Gao 2005; Koch and Tampe, 2006; Sullivan et al., 2006).

The $\propto 3$ chain folds into an Ig domain, a part of the amino acid sequence, which is conserved among all class I molecules. This segment contains a loop serving as binding site for CD8. Near the carboxy terminal end is a stretch of approximately 25 hydrophobic amino acids that traverses the lipid bilayer of the plasma membrane. Next to this there are 30 residues located in the cytoplasm, a cluster of basic amino acids that interact with the phospholipid head groups of the inner sheet of the lipid bilayer, thereby, anchoring the MHC molecule in the plasma membrane (Flutter and Gao, 2005; Koch and Tampe, 2006; Sullivan et al., 2006), see Figure 3.

The $\beta_{2}$-microglobulin encoded by a gene outside the MHC is the light chain combining with a class I molecule, interacting non-covalently with the $\propto 3$ segment. The $\beta_{2}$-microglobulin is the invariant component of all class I molecules.

The purpose of the second-mentioned surveillance system is to identify abnormal body cells, such as those infected with viruses, or those which have turned malignant. When such cells display unfamiliar peptide antigens, e.g. fragments of viral proteins, they are attacked and destroyed (Flutter and Gao, 2005; Germain, 1995; Hamilos, 1989;Jones et al., 1988).

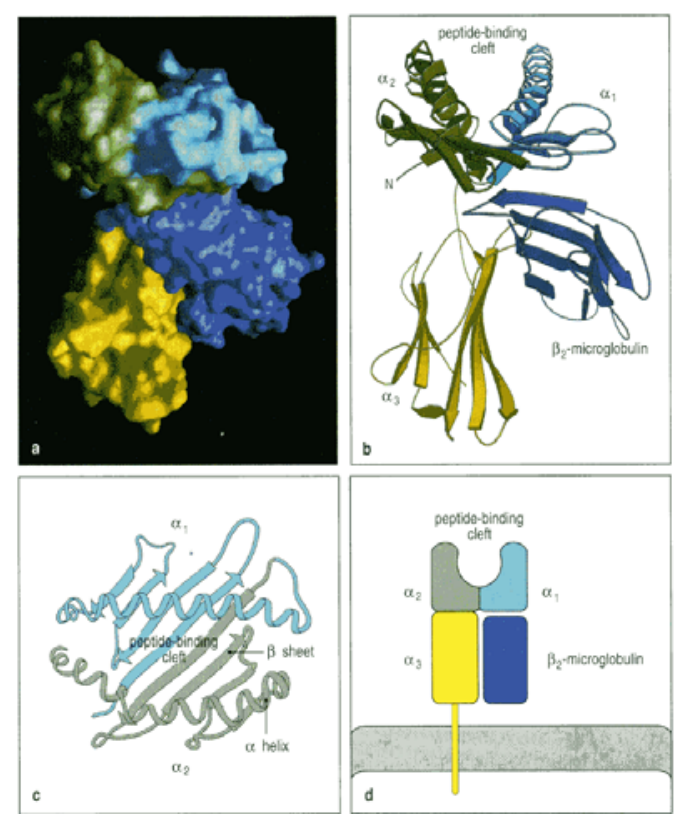

Figure 3. The structure of MHC class I protein with bound to $\beta_{2}$ microglobulin as determined by X-ray crystallography. 
Panel (a) shows a computer graphic representation of the two subunit-comples, of human MHC class I molecule, panel (b) a ribbon diagram. Panel (c) gives a view of its tertiary structure, looking down on the molecule from above; the sides of the cleft are formed by the inner faces of the two $\propto$ helices. Panel (d) shows a schematic representation of the extracellular parts of an MHC class I molecule (Janeway et al., 1999).

\subsubsection{MHC class II molecules}

The molecules of the MHC class II constitute a heterodimer that contains an $\propto$ chain and a $\beta$ chain. The two portions alpha helixes are on a beta-pleated, both form a cleft, that allows the union specifies of peptides. This portion presents a variable amino acid sequence with respect to the rest of the molecule (Castellino, 1997).

The molecules of the MHC class II are DP, DQ and DR, although the highly polymorphic peptides are recognized by receptors of CD4 T cells. The pathogens and extracellular proteins phagocyted in intracellular vesicles derive the peptide antigens that present these molecules class II (Castellino, 1997).

MHC class II molecules expressed in the thymus also have a vital role in the intrathymic maturation of CD4 T cells. MHC class II molecules are expressed constitutively on antigen-presenting cells, including B lymphocytes, macrophages, and dendritic cells (Lundberg, 1997).

They are also expressed on the epithelial cells of the thymus and their expression can be induced on other cells, principally by the cytokine interferon- $y$. T cells also express MHC class II molecules when they are activated. Expression of the genes encoding the $\alpha$ and $\beta$ chains of MHC class II molecules must be strictly coordinated and is under complex regulatory control. The regulation of MHC class II gene expression is not fully understood as it involves the action of transcription factors that are defined only in part. The existence of these transcription factors and a means of identifying then were first suggested by the study of patients with MHC class II deficiency. Many diseases are associated with the MHC class II region (Jones et al., 2006; Germain, 1995; Hamilos et al., 1989).

The structure of class II differs from that of class I in the absence of remainders of tyrosines in the end of the cleft. These are replaced by remainders of glycine and valine that have so large minor (Castellino, 1997; Lundberg and McDevitt 1992). 


\subsubsection{MHC in Callithrix jacchus and others New World monkeys (NWM)}

The New World Monkey (NWM) and Old World simians radiated about 58 millions years ago (Ciochon and Chiarelli, 1980). The comparison with the 'classical' (HLA-A, $B$ and $C$ ) and three conserved 'non-classical' (HLA-E, F and G) MHC class I genes of apes and monkeys have shown in their differention been preserved during primate evolution. These six human genes have orthologues with African apes. Several MHC class I genes of Asian apes have shown significant differences from the human genes which continues with the Old World monkeys, and even more so in the New World monkeys, where $E$ and $F$ are the only human gene orthologues. The locus $C$ is confined to humans and is the result of a recent duplication of the $B$ locus (Boyson et al., 1993; Boyson et al., 2001; Knapp et al., 1998).

Common marmoset has an evolutionary distance from humans of 55 million years (Bontrop et al., 1995). Immunological studies have shown that its immune system is a particularly good model when compared to other primates for testing antibody specificity and recognition (Genian and Hauser, 2001). Others NWM have been studied as animal models in multiples diseases, such as Aotus, Saguinus, Saimiri, principally, and other species (Cadavid et al., 1997; Patarroyo et al., 1987; Diaz et al., 2000; Middleton et al., 2004).

Homologies of MHC class I genes are present in all Catarrhini (old world apes and the human). However, the Callitrichinae (tamarins and common marmoset) are exceptions to the rules of MHC stability. This specie Callithrix jacchus (common marmoset) has Caja-G 01 until Caja-G 05 and Caja-E genes MHC class I, these sequences gene have been compared with others NWM which form separated clusters in the phylogenetic tree (Cadavid et al., 1997). Their cDNAs indicate that there is no orthology between MHC class I loci in the genera of this phyletic group, furthermore, their MHC class exhibits limited variability, probably the result of the recent origin of these loci (Cadavid et al., 1997).

MHC class II region genes have limited polymorphism, it has been expanded by reactivation of pseudogene segments as a result of exon shuffling (Doxiadis et al., 2006). This limited variability may contribute its susceptibility to particular bacterial infections (klebsiella, bordetella, clostridium and shigella) (Potkay, 1992). These encode the evolutionary equivalents of human HLA-DR and DQ molecules (Bontrop et al., 1999), but the Caja DP region has been inactivated (Antunes et al., 1998). The 
Caja DR region contains only three loci: Caja-DRB*W12, DRB*W16 and DRB1*03 (Wu et al., 2000; Antunes et al., 1998; Prasad et al., 2006).

Several Aotus species (nancymaae, nigriceps and vociferans) have been studied for MHC class II. This work has revealed a high homology between MHC-DR and HLADRB (Suarez et al., 2006; Niño-Vasquez et al., 2006). The sequence of MHC class I of exon 2 and 3 which was divided into two groups Aog1 and Aog2, their analyses showed that displays to similar characteristics to Catarhini's classical loci. This sequence was found as a processed pseudogene (Aona PS2) (Cadavid et al., 1997). Most genetic and functional work on class I genes has been on the cotton top tamarin (Saguinus Oedipus) (Cadavid et al., 1997; Shneider et al., 1993); this species has revealed only 11 different expressed class I molecules. The selective pressure on MHC class I molecules and the peptide binding of these molecules may drive the generation of MHC class I polymorphism, furthermore, the gene conversion occurring in genetic exchange not only occurrs between alleles of locus but also between loci (Watkins et al., 1991; Watkins et al., 1991; Watkins et al., 1996). Alvarez-Tejado et al. (1998) found data suggesting that sequences of MHC (MhcSaoe $\mathrm{CR}^{*} 01$ and $\mathrm{CR}^{*} 02$ ) may be related to the Mhc-C locus. However, they share only a few of the conserved residues (from gorilla and human) which suggests that the relationship to an ancestor of the MHC-C lineage is very distant or is a product of convergent evolution to perform a $C$ locus related to function, and the locus $C$ is the result of a duplication of the B locus in great apes and human (Boyson et al., 1996). The pseudogenes found in these species, PS1 and PS2 have been characterized from class I mRNA not functionally expressed template. PS2 has been found in Callithrix jacchus and Aotus trivirgatu (Cadavid et al., 1996). Other processed have found likely true pseudogenes (Gp12, Gp13, Gp14) and partial pseudogenes (Gp15, Gp16, Gp17, Gp18, Gp19 and Gp20) (Cadavid et al., 1999).

The characterization and phylogenetic analysis of sequences of the MHC class I of new world monkey Saimiri sciureus showed that they are related to HLA class 1 genes (HLA $A$ and $G$ ), and the structure and the organization of one clone was similar to HIA-A 2 (Pascalis et al., 2003). 


\subsubsection{MHC in other primates}

Several non-human primates of the Old World monkey (OWM) have been used in biomedical research as a model, for multiples diseases: (see following Table 2.3.5 Adams and Parham, 2000). Some of these diseases (immuno-related) have needed the characterization of their MHC system (Otting et al., 2002).

\section{Table 2.3.5 Adams and Parham, 2001.}

"The relationship of non-human primate $M H C$ class I loci to those expressed in humans. Orthologous loci are indicated by blue shading, homologous loci are indicated by yellow shading. Hatched coloration indicates the presence of an additional homologous locus within that species".

\begin{tabular}{|c|c|c|c|c|c|c|c|c|c|c|}
\hline & Species & Common name & HLA-A & $H L A-B$ & HLA-C & $H L A-E$ & $H L A-F$ & $H L A-G$ & $H L A-H$ & Unrelated \\
\hline \multirow{3}{*}{ 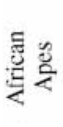 } & Pan troglodytes & Common chimpanzee & 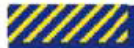 & & & & & & & $?$ \\
\hline & Pan paniscus & Bonobo & & & & n.d & n.d. & n.d. & & $?$ \\
\hline & Gorilla gorilla & Gorilla & 10112 & & & & & & & $?$ \\
\hline \multirow{3}{*}{ 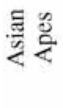 } & Pongo pygmaeus & Orangutan & & & & & n.d. & & $?$ & $?$ \\
\hline & Hylobates Lar & Gibbon & & & $?$ & n.d. & n.d. & n.d. & $?$ & $?$ \\
\hline & Macaca mulatia & Rhesus macaque & & & $?$ & & & & $?$ & $?$ \\
\hline \multirow{5}{*}{ 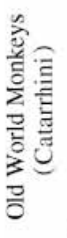 } & Macaca fascicularis & $\begin{array}{l}\text { Crab-eating macaque or } \\
\text { Cynomologous monkey }\end{array}$ & n.d. & & ? & & n.d. & n.d. & $?$ & $?$ \\
\hline & Macaca arctoides & Stump-tailed macaque & n.d. & & $?$ & n.d. & n.d. & n.d. & ? & $?$ \\
\hline & Papio cynocephalus & Yellow baboon & & & $?$ & & n.d. & n.d. & $?$ & $?$ \\
\hline & $\begin{array}{l}\text { Papio cynocephalus } \\
\text { (hamadryas) anubis }\end{array}$ & Olive baboon & & & $?$ & n.d. & n.d. & n.d. & $?$ & $?$ \\
\hline & Cercopithecus aethiops & African green monkey & $?$ & $?$ & $?$ & n.d. & n.d. & & $?$ & n.d. \\
\hline \multirow{11}{*}{ 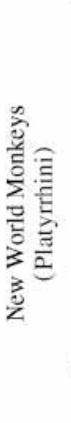 } & Ateles belzebuth & Long-haired spider monkey & $?$ & $?$ & $?$ & & n.d. & $?$ & $?$ & \\
\hline & Ateles fusciceps & Brown-headed spider monkey & $?$ & $?$ & $?$ & & n.d. & ? & $?$ & n.d. \\
\hline & Pishecia pithecia & White-faced saki & $?$ & $?$ & $?$ & & n.d. & $?$ & $?$ & \\
\hline & Saimiri sciureus & Common squirrel monkey & $?$ & $?$ & $?$ & n.d. & n.d. & ? & $?$ & \\
\hline & Aotus trivirgatus & Owl monkey, Douroucouli & $?$ & $?$ & $?$ & & n.d. & $?$ & $?$ & \\
\hline & Saguinus mystax & Moustached tamarin & $?$ & $?$ & ? & n.d. & n.d. & $?$ & ? & \\
\hline & Saguinus geoffroyi & Geoffrey's tamarin & $?$ & $?$ & $?$ & n.d. & n.d. & ? & $?$ & \\
\hline & Saguinus fuscicollis & Brown-headed tamarin & $?$ & $?$ & ? & n.d. & n.d. & $?$ & $?$ & \\
\hline & Saguimus oedipus & Cotton-top tamarin & $?$ & $?$ & ? & & & $?$ & $?$ & \\
\hline & Leontopithecus rosalia & Golden lion tamarin & $?$ & $?$ & $?$ & n.d. & n.d. & $?$ & $?$ & \\
\hline & Callithrix jaccus & White-tufted-ear marmoset & $?$ & $?$ & $?$ & & n.d. & $?$ & $?$ & \\
\hline
\end{tabular}

Macaca mulatta monkey is known like rhesus monkey, their MHC class I region has been described to have a length of $3.28 \mathrm{Mb}$ and 64 genes: 23 are expressed and 41 pseudogenes, however, have been difficult to define if has identified orthologous of the human HLA A, B, E, F and G genes (Boyson et al., 1996; Boyson et al., 1995; Go et al., 2002; Otting et al., 2002; Daza-Vementa et al., 2004; Otting et al., 2005; Shiina et al., 2006). The rhesus monkeys, and possibly the Old World monkeys in general diverged from humans 27-30 Million years (Myr) ago. Rhesus monkeys were found to not have the pair of MHC-B and MHC-C but many repeated genes similar to $\mathrm{MHC}$ - 
B. These results support the inference that MHC-B and MHC-C duplicated after the divergence between apes and Old World monkeys (Fukami-Kobayashi et al., 2005; Kulski et al., 2005; Kulsi et al., 2004; Kulski et al., 2002).

In Chimpanzee (Pan troglodytes) has been found the structural and/or functional orthologues for all human $H L A$ genes (HLA-A/B/C/E/F/G vs. Patr-A/B/C/E/F/G) (Anzai et al., 2003; Adams and Parham, 2001). However, it has been assumed to be closely linear to that of human, although, the genomic architecture of chimp MHC is unknown (Anzai et al., 2003).

The work carried out on chimpanzee, rhesus monkey and human has revealed that MHC-B and MHC-C duplicated 22.3 Myr ago, and the ape MICA and MICB duplicated 14.1 Myr ago (Fukami-Kobayashi et al., 2005; Kulski et al., 2005; Kulski et al., 2002).

\subsubsection{Evolution}

The diversity was created and maintained by evolutionary forces: selection, mutation, genetic drift and migration. The frequency dependent selection and heterozygote advantage were two types of balancing selection that have been suggested to explain MHC allelic diversity (Harlt, 2001).

The MHC shows a high degree of polymorphism (100 times greater than the genome average, i.e. a $10 \%$ difference between any two unrelated individuals). One hypothesis holds that the MHC class I originated first as a result of a recombination between an immunoglobulin-like C-domain and the peptide-binding domain of an HSP70 heat-shock protein (Flajnik et al., 1991). A phylogenetic analysis supports a relationship between the MHC class II-alpha chain and $\beta_{2}$ microglobulin and between the MHC class II beta-chain and the class I alpha chain (Hughes et al., 1993).

About 370 million years or more have been suggested for the physical linkage of $\mathrm{MHC}$ regions because of the presence of all three classes of I, II and III genes in the amphibian Xenopus (Kaufman et al., 1990).

There is no single definite candidate for a primordial MHC gene. According to one hypothesis, the MHC class II evolved first, whereas to a second hypothesis the MHC loci do not always exist in a single linked cluster as they do in mammals, but can be found in two or multiple clusters (Miller et al., 1994; Bingulac-Popovic et al., 1997). In vertebrates, the immune system is an adaptive defense system with its components MHC, TCR and immunoglobulin (Ig) genes. In invertebrates, however, the innate 
immune system is the only defensive system. These main components of the adaptive immune system are missing not only in invertebrates but also in primitive 'jawless' vertebrates (Matsunaga et al., 1998; Klein et al., 1998).

MHC class I genes do not show an orthologous (i.e., homologous by descent from a common ancestral locus) relationship between mammals of different orders whereas orthologous relationships have been found, among mammalian class II loci 6 (Huges and Nei, 1990). The HLA-C locus has been found only in the human, gorilla and chimpanzee but not in other monkeys (Boyson et al., 1996). The MHC class I proteins however, have been shown to be present in Callithrix jaccus (Cadavid et al., 1997).

New genes are created by repeated gene duplication and some duplicate genes are maintained in the genome for a long time, while others are deleted or become nonfunctional by deleterious mutations. This concept disagrees with the earlier idea that MHC diversity and evolution are governed by concerted evolution of the multigene families of the major histocompatibility complex (MHC) genes and the immunoglobulin (lg) genes. The alleles seem to have a fast turnover rate. The lack of correspondence between the alleles in human and chimpanzee suggests that five million years of separation have been sufficient to reconfigurate MHC alleles. This means that the alleles are constantly undergoing modifications during their transspecies evolution (Parham and Otta, 1996).

\subsection{Framework genes hypothesis}

The framework hypothesis can explain the non-orthologous class I sequences are occupy homologous locations to the conserved genes; the identification of non-class I genes allows a comparative map to be drawn, which shows the orthologous class I region. That was showed in the class I region between human and mouse, but the definition of the class I framework applies to all mammals. The class I framework could help define the ancestral MHC, by trancing the conserved genes earlier in the phylogeny and their linkage with MHC genes (Amadou, 1999).

The proximal to the distal part of the class I region, conserved genes mapped in several species (Human, mouse, rat, chimpanzee, rhesus monkey, lemur, pig, chicken) are: BAT1, POU5F, TCF19, GNL1, TRIM26, TRIM39, TCTEX5 and MOG. 
2.4.1 ATP6V1G2: ATPase, $\mathrm{H}+$ transporting, lysosomal 13kDa, V1 subunit G2 Gene aliases: NG38; ATP6G; VMA10; ATP6G2

A vacuolar ATPase (V-ATPase) is encoded by the ATP6V1G2 gene, it is an enzyme that mediates acidification of intracellular compartments of eukaryotic cells. It is necessary for intracellular processes such as protein sorting, zymogen activation, receptor-mediated endocytosis, and synaptic vesicle proton gradient generation. The composition of V-ATPase protein is a cytosolic V1 domain and a trans-membrane V0 domain. The $\mathrm{V} 1$ domain has three $\mathrm{A}$ and three $\mathrm{B}$ subunits, two $\mathrm{G}$ subunits plus the $\mathrm{C}$, $D, E, F$, and $H$ subunits and contains the ATP catalytic site. The V0 domain consists of five different subunits: a, c, c', c", and d. Vacuolar type ATPases are involved in bone resorption, glycosylation in the Golgi, degradation of cellular debris in lysosomes, and the processing of endocytosed receptor-ligand complexes. By sequence analysis, the ATP6G2 gene was mapped to chromosome 6p21.3, approximately $1 \mathrm{~kb}$ telomeric to NFKBIL1 and centromeric to BAT1. The full-length 118-amino acid protein is $82 \%$ similar to the cow protein. The other isoform encodes a 77-amino acid protein. RT-PCR analysis detected expression in lymphocytic but not monocytic or macrophage-like cell lines (Neville and Campbell, 1999).

\subsubsection{BAT1: HLA-B associated transcript 1}

Gene aliases: D6S81E, UAP56

This gene encodes the protein by a member of the DEAD protein family of ATPdependent RNA helicases. By chromosome walking with overlapping cosmids isolated a 435-kb DNA segment that was centromeric to HLA-B. This gene lies between TNF and HLA-B. The gene contains 10 exons spanning about $10 \mathrm{~kb}$ of genomic DNA and encodes a 428-amino acid protein detected with three different length mRNAs $(4.1,17$, and $0.9 \mathrm{~kb})$ in all tissues analyzed, although at different relative levels. Its cellular functions include initiation of translation, RNA splicing, and ribosome assembly. UAP56 (BAT1) is an essential splicing factor that is recruited to the pre-mRNA dependent on U2AF65 and is required for the U2 snRNP-branch point interaction. UAP56 is a member of the DEAD box family of RNA-dependent ATPases, which mediate ATP hydrolysis during several steps of pre-mRNA splicing. Proteins of this family have nine conserved amino acid motifs but differ at their amino and carboxyl ends. From studies of other family members, the first block is involved in ATP binding, the fifth block may be an ATPase, the sixth block is needed for RNA 
helicase activity, and the ninth block is involved with ATP hydrolysis-independent RNA interactions during unwinding (Spies et al., 1989; Peelman et al., 1995; Fleckner et al., 1997; Allcock et al., 2001; Price et al., 2004).

\subsubsection{TCF19: transcription factor 19 (SC1)}

Gene aliases: SC1; SC1-1

The complete genomic structure of a $5.5 \mathrm{~kb}$ DNA comprises three exons, generating a $2.5 \mathrm{~kb}$ transcript. The TCF19 gene spans a 3.2 stretch of DNA between POU5F1 and $S$ to a $0.2-\mathrm{Mb}$ region between HLA-C, about $130 \mathrm{~kb}$ telomeric of HLA-C and about $600 \mathrm{bp}$ from each other. It is mammal-specific and preferentially expressed in the G1-s. This gene encodes a 359 amino acid protein, and is a possible transactivating factor for the later stage of cell cycle progression (Ku et al., 1991; Krishnan et al., 1995; Teraoka et al., 2000).

\subsubsection{POU5F1: POU domain, class 5, transcription factor 1}

Gene aliases: OCT3; OTF3; OTF4; Oct4; MGC22487

The genomic span of the POU5F1 gene is $45 \mathrm{~kb}$, and the localization is the $6 \mathrm{~kb}$ HLA fragment. This localization was confirmed by linkage of a Random Fragment Lenthg Polymorphic (RFLP) in 9 CEPH families, indicating tight linkage to HLA-A, $-B,-C$, and -DR. This gene is encoded the protein by the members of the POU (representing a homeodomain protein family of the founder members which are Pit-1, Oct-1/2 and Unc-86) homeodomain protein OCT/Oct-3 (where OCT stands for octamer binding protein) is an embryonic transcription for expressed in oocytes, embryonic stem and embryonic carcinoma cells. The octamer cis-acting transcriptional regulatory motif (ATGCAAAT) is found in enhancers and promoters of many genes which are expressed either ubiquitously or in tissue-specific fashion (Takeda et al., 1992; Sylvester et al., 1994).

2.4.5 TRIM family: The tripartite motif (TRIM) protein family.

The TRIM is composed of three zinc-binding domains, a RING (R), a B box type (B1) and a B-box type 2 (B2), followed by a coiled coil (CC) region. Their genes are implicated in a variety of processes, such as development and cell growth and are involved in several human diseases. However, little is known about the biological and molecular mechanisms mediated by the TRIM genes. (Reymond et al., 2001; Reddy 
et al., 1992; Borden, 1998). In this study, TRIM10, TRIM15, TRIM26 and TRIM39 were found.

\subsubsection{TRIM10}

Gene aliases: RNF9; HERF1; RFB30

By screening a human chromosome 6-specific library with a B30.2 domain-encoding exon that had been mapped to 6 p21.3 as the probe, a cDNA encoding RFB30 was obtained. Sequence analysis predicted that the 481-amino acid protein contains a RING finger-B box domain encoded by exon 1 , a coiled-coil domain encoded by exons 2 through 6, and a B30.2 domain encoded by exon 7. In embryonic mice, expression was detected on day 11.5 at the beginning of erythropoiesis. It was determined that the TRIM10 gene contains at least seven exons (Henry et al., 1997; Harada et al., 1999).

\subsubsection{TRIM39 Gene aliases: TFP; RNF23; MGC32984}

The deduced 519-amino acid protein, $98 \%$ identical to the 489-amino acid mouse sequence, contains a RING finger B-box coiled-coil (RBCC) domain and a C-terminal B30.2 domain. Northern blot analysis revealed nearly ubiquitous expression of a 3.4$\mathrm{kb}$ transcript, with strongest expression in the testis. By genomic sequence analysis, it was determined that the RNF23 gene contains at least 8 exons (Orimo et al., 2000).

\subsubsection{TRIM26 Gene aliases: AFP; RNF95; ZNF173}

Positional cloning of short fragment CDNA sequences from the class I region of the human major histocompatibility complex (MHC) was performed using a hybridization selection approach. Full-length cDNA clones were isolated and also partial genomic clones encoding a protein with two domains rich in cysteine and histidine similar to those characteristic of metal-dependent DNA-binding proteins. The predicted protein also contains a domain thought to form a coiled-coil, and possibly to promote dimerization. A third feature of the predicted protein is a polyglutamic acid region near the carboxyl terminus. Because of these properties, the gene product was named acid-finger protein, AFP (a designation used originally for alpha-fetoprotein AFP). Although the biological role of AFP is unknown, one potential function is the 
binding of nucleic acids. The gene is expressed in multiple tissues and conserved among mammals (Chu et al., 1995).

\subsubsection{TCTEX5}

Gene aliases: HCGV; HCG-V; TCTE5; TCTEX5; MGC125741; MGC125742; MGC125743, PPP1R11.

The gene is located within the major histocompatibility complex class I region on chromosome 6 . This gene encodes a specific inhibitor of protein phosphatase-1 (PP1) with a differential sensitivity toward the metal-independent and metaldependent forms of PP1. Alternative splicing results in two transcript variants encoding different isoforms. Other alternatively spliced transcripts have been described, but their full length sequences have not been determined (Yoshino et al., 1998).

\subsubsection{MOG Myelin-oligodendrocyte glycoprotein}

Gene aliases: MGC26137

The primary nuclear transcript of the human MOG gene, extending from the putative start of transcription to the site of poly (A) addition, is 15,561 nucleotides in length. The gene contains 8 exons, separated by 7 introns. The introns vary in size from 242 to $6,484 \mathrm{bp}$ and contain numerous repetitive DNA elements, including 14 Alu sequences within 3 introns. The human MOG gene lies $60 \mathrm{~kb}$ telomeric to HLA-F in a head-to-head orientation. The product of this gene is a membrane protein expressed on the oligodendrocyte cell surface and the outermost surface of myelin sheaths. Due to this localization, it is a primary target antigen involved in immune-mediated demyelination. This protein may be involved in completion and maintenance of the myelin sheath and in cell-cell communication. Myelin-oligodendrocyte glycoprotein is found on the surface of myelinating oligodendrocytes and external lamellae of myelin sheaths in the central nervous system. The N-terminal, extracellular region of MOG has characteristics of an immunoglobulin variable domain and strong homology with the N-terminus of butyrophilin, a protein expressed in the lactating mammary gland. Alternatively spliced transcript variants encoding different isoforms have been identified (Pham-Dinh et al., 1995; Roth et al., 1995). 


\section{Materials}

\subsection{Chemicals}

\section{Reagents}

- Acetic acid glacial

- Agar-agar

- Agarose LE seakem

- Albumin, bovine

- Bacto-yeast-extract

- Bact- peptone

- Boric acid

- Bromphenol blue

- BSA fraction $V$

- Carbon dioxide

- N,N-dimethyl formamide

- Ethylene diamino tetraacetic acid (EDTA)

- Ethanol

- Ethidium bromide solution $1 \%$

- Formaldehyde $37 \%$

- Formamide, deionized

- Glucose

- Glycogen 20 mg/ml

- Hydrocloric acid $37 \%$

- Isopropanol

- Isopropyl-ß-D-pyranoside thiogalacto (IPTG)

- Magnesium chloride

- Magnesium sulfate

- 2-Propanol

- Polyvinyl pyrrolidone

- Potassium acetate

- Potassium chloride

- Sodium acetate $3 \mathrm{H}_{2} \mathrm{O}$

- Sodium chloride

- Sodium hydroxide

- Sodium iodide

\section{Source}

Roth

Roth

Biozym

Sigma

Gibco BRK GhbH

Gibco BRK GhbH

Roth

Servo

Sigma

Merck

Roth

Sigma

Roth

Roth

Roth

Roth

Merck

Merck

Roth

Roth

Biomol

Merck

Merck

Roth

Merck

Roth

Merck

Roth

Roth

Roth

Roth 
- Sodium dodecyl sulfate (SDS) ultrapure Roth

- Tri-sodium citrate $\cdot 2 \mathrm{H}_{2} \mathrm{O}$ Roth

- Tris base $99.9 \%$ Roth

- Tryptone/peptone of casein Roth

- 5-Brom-4-chlor-3-indolyl-ß-D- galactoside (X-gal) Roth

- Xylene cyanol Merck

- Yeast extract Roth

\subsection{Buffers and other solutions}

\subsubsection{Ammonium acetate $10 \mathrm{M}$}

$385.4 \mathrm{~g}$ ammonium acetate dissolved in $150 \mathrm{ml} \mathrm{H}_{2} \mathrm{O}, \mathrm{H}_{2} \mathrm{O}$ added to $500 \mathrm{ml}$

\subsubsection{Denaturation solution}

\section{$1.5 \mathrm{M} \mathrm{NaCl}$}

$0.5 \mathrm{M} \mathrm{NaOH}$

\subsubsection{Denhardt's solution $100 x$}

$10 \mathrm{~g}$ Ficoll 400

$10 \mathrm{~g}$ polyvinyl pyrrolidone

$10 \mathrm{~g}$ bovine serum albumin (BSA fraction $\mathrm{V}$ ), $\mathrm{H}_{2} \mathrm{O}$ to $500 \mathrm{ml}$

Filters sterilized and stored at $-20^{\circ} \mathrm{C}$ as $25 \mathrm{ml}$ aliquots in $1 \%$ BSA fraction $\mathrm{V}$

\subsubsection{DNA-lysis buffer}

$50 \mathrm{mM}$ Tris- $\mathrm{HCl} \mathrm{pH} 8.0$

100 mM EDTA pH 8.0

$0.5 \%$ SDS

\subsubsection{EDTA 0.5M pH 8.0}

$186 \mathrm{~g} \mathrm{Na} \mathrm{Na}_{2}$ EDTA $\cdot 2 \mathrm{H}_{2} \mathrm{O}$ dissolved in $700 \mathrm{ml} \mathrm{H}_{2} \mathrm{O}$

$\mathrm{pH}$ adjusted to 8.0 with $10 \mathrm{M} \mathrm{NaOH}$

$\mathrm{H}_{2} \mathrm{O}$ added to 1 liter 


\subsection{6 $\mathrm{HCl} 1 \mathrm{M}$}

$91.38 \mathrm{ml} \mathrm{H}_{2} \mathrm{O}$

$86.2 \mathrm{ml}$ concentrated $\mathrm{HCl}$

\subsubsection{KCl $1 \mathrm{M}$}

$74.6 \mathrm{~g} \mathrm{KCl}$

$\mathrm{H}_{2} \mathrm{O}$ to $100 \mathrm{ml}$

\subsubsection{Loading for buffer}

$0.25 \%$ Bromophenol blue

$0.25 \%$ Xylene cyanol

$15 \%$ Ficoll 400

50 mM EDTA pH 8.0

\subsubsection{Lysis solution I P1}

50 mM Glucose

25 mM Tris- $\mathrm{HCl} \mathrm{pH} 8.0$

10 mM EDTA pH 8.0

\subsubsection{Lysis solution II P2}

$0.2 \mathrm{M} \mathrm{NaOH}$

$1 \%$ SDS

\subsubsection{Lysis solution III P3}

3 M Potassium acetate

Adjusted with concentrated $\mathrm{HCl}$ to $\mathrm{pH} 5.5$

\subsubsection{2 $\mathrm{MgCl}_{2} 1 \mathrm{M}$}

$20.3 \mathrm{~g} \mathrm{MgCl}_{2} \bullet 6 \mathrm{H}_{2} \mathrm{O}$

$\mathrm{H}_{2} \mathrm{O}$ to $100 \mathrm{ml}$

\subsubsection{3 $\mathrm{MgSO}_{4} \mathrm{1M}$}

$24.6 \mathrm{~g} \mathrm{MgSO}_{4}$

$\mathrm{H}_{2} \mathrm{O}$ to $100 \mathrm{ml}$ 


\subsubsection{4 $\mathrm{NaCl} 5 \mathrm{M}$}

$292 \mathrm{~g} \mathrm{NaCl}$

$\mathrm{H}_{2} \mathrm{O}$ to 1 liter

\subsubsection{NaOH}

$400 \mathrm{~g} \mathrm{NaOH}$ dissolved in $450 \mathrm{ml} \mathrm{H}_{2} \mathrm{O}$

$\mathrm{H}_{2} \mathrm{O}$ added to 1 liter

\subsubsection{Pre-hybridization solution}

20X SSC $75 \mathrm{ml}$

50X Denhardt's $25 \mathrm{ml}$

$10 \%$ SDS $25 \mathrm{ml}$

$\mathrm{H}_{2} \mathrm{O}$ added to $250 \mathrm{ml}$

\subsubsection{Sodium acetate $3 \mathrm{M}$}

$408 \mathrm{~g}$ sodium acetate dissolved $3 \cdot \mathrm{H}_{2} \mathrm{O}$ in $800 \mathrm{ml} \mathrm{H}_{2} \mathrm{O}, \mathrm{H}_{2} \mathrm{O}$ added to 1 liter, $\mathrm{pH}$ adjusted to 4.8 or 5.2 with $3 \mathrm{M}$ acetic acid

\subsubsection{SSC (sodium chloride/sodium citrate), $20 \mathrm{x}$}

$3 \mathrm{M} \mathrm{NaCl}(175 \mathrm{~g} /$ liter $)$

$0.3 \mathrm{M}$ Sodium citrate $2 \mathrm{H}_{2} \mathrm{O}$ (88 g/liter)

\subsubsection{Sodium lodine}

$121 \mathrm{~g} \mathrm{Nal}$

$\mathrm{H}_{2} \mathrm{O}$ added to $250 \mathrm{ml}$

\subsubsection{0x SSC (Sodium chloride sodium citrate)}

$3 \mathrm{M} \mathrm{NaCl}$

$0.3 \mathrm{M}$ sodium acetate

Adjust with $\mathrm{HCl}$ concentrated to $\mathrm{pH} 7.0$

3.2.21 TBE (tris/borate/EDTA) electrophoresis buffer

10X stock solution, 1 liter:

$108 \mathrm{~g}$ tris base $(890 \mathrm{mM})$ 
$55 \mathrm{~g}$ boric acid (890 $\mathrm{mM})$

$40 \mathrm{ml} 0.5 \mathrm{M}$ EDTA, pH 8.0

\subsubsection{TrisHCl [Tris (hydroxymethyl) aminomethano], 1 M}

$121 \mathrm{~g}$ tris base dissolved in $800 \mathrm{ml} \mathrm{H}_{2} \mathrm{O}$

Adjust to desired $\mathrm{pH}$ with concentrated $\mathrm{HCl}$

Mix and add $\mathrm{H}_{2} \mathrm{O}$ to 1 liter

Approximately $70 \mathrm{ml}$ of $\mathrm{HC}$ is needed to achieve $\mathrm{pH} 7.4$ solution and approximately $42 \mathrm{ml}$ for 8.0.

IMPORTANT NOTE: The $\mathrm{pH}$ of tris buffer changes significantly with temperature, decreasing approximately $0.0028 \mathrm{pH}$ units per $1^{\circ} \mathrm{C}$. Tris-buffered solutions should be adjusted to the desired $\mathrm{pH}$ at the temperature used. Because the $\mathrm{pK}_{\mathrm{a}}$ of tris is 8.08 , it should not be used as a buffer below $\mathrm{pH} \sim 7.2$ or above $\mathrm{pH} \sim 9.0$.

\subsubsection{0x Tris-phosphate-buffer}

$108 \mathrm{~g}$ tris base

$15.5 \mathrm{ml}$ phosphoric acid (85\%, $1.679 \mathrm{~g} / \mathrm{ml})$

$40 \mathrm{ml}$ 0.5M EDTA pH 8.0

\subsection{Media}

\subsubsection{LB Medium (Luria-Bertani):}

$1 \%$ Pepetone 140

$0.5 \%$ Yeast extracts

$1 \% \mathrm{NaCl}$

$1.5 \%$ Agar to solid medium

\subsubsection{SOB Medium}

$2 \%$ Select peptone 140

$0.5 \%$ Yeast extract

$10 \mathrm{mM} \mathrm{NaCl}$

$2.5 \mathrm{mM} \mathrm{KCl}$

$10 \mathrm{mM} \mathrm{MgCl} 2$

$10 \mathrm{mM} \mathrm{MgSO}_{4}$ 


\subsubsection{SOC Medium}

SOB Media

$20 \mathrm{mM}$ Glucose

\subsubsection{Antibiotic solutions}

\subsubsection{Ampicillin}

$50 \mathrm{mg}$ Ampicillin to 1 liter medium

Before dilution, the ampicillin solution must be sterilized by filtration

\subsubsection{Chloramphenicol}

$20 \mathrm{mg}$ to 1 liter medium

Before dilution, the chloramphenicol solution must be sterilized by filtration

\subsection{Commercials kits}

- $\quad$ Big Dye Terminator version 1.1, Applied Biosystems

- Cycle Sequencing Kit, Applied Biosystems

- Megaprime DNA Labelling, Promega

- Prime-a-Gene Labeling System, Promega

- QIAamp DNA Mini Kit, Qiagen

- QIAquick Gel Extraction Kit, Qiagen

- QIAquick PCR Purification Kit, Qiagen

- System, dCTP, Amersham

- Taq PCR Care Kit, Qiagen

\subsection{Enzymes}

- BamHI

10.000 U New England Biolabs (NEB)

- Biotherm Taq-Polymerase $1000 \mathrm{U}$ Genecraft

- ECORI

$10.000 \mathrm{U} \quad \mathrm{NEB}$

- HindIII

$10.000 \cup \quad$ NEB

- Not I

$10.000 \cup \quad$ NEB

- Pstl

$10.000 \cup \quad N E B$ 
- Ribonuclease A

- RNAase A

- Sequenace

- T4-DNA Ligase
Roth

Roth

Perkin Elmer

20.000U NEB

\subsection{Oligonucleotides primers for PCR: sequences and annealing temperatures} applied.

\begin{tabular}{|c|c|c|}
\hline Primer & Primer sequence & $\begin{array}{c}\text { Annealing } \\
\text { temperature }{ }^{\circ} \mathrm{C}\end{array}$ \\
\hline T7 & 5'aatacgactcactataggg 3' & 52 -(touchdown) \\
\hline pTARBAC2 & 5'cttacgcagggcatccatt 3' & 52 -(touchdown) \\
\hline pTARBAC3 & 5'acatttaggtgacactatag 3' & 52 -(touchdown) \\
\hline pTARBAC4 & 5'atacaaagaaacgtacggc 3' & 52 -(touchdown) \\
\hline T7.29 & 5'gccgctaatacgactcactatagggagag 3' & 60 \\
\hline SP6.26 & 5'ccgtcgacatttaggtgacactatag 3' & 60 \\
\hline $\mathrm{K}_{18 T 3^{1}}$ & 5'agtacttataggaattattacca 3' & 52 \\
\hline $\mathrm{K}_{183^{1}}$ & 5'gttgctttattgtgtcactagtc 3' & 52 \\
\hline $\mathrm{POU} \mathrm{F}^{2}{ }^{2} \mathrm{~F}$ & 5'atggcgggacacctggcttcgg 3' & 58 \\
\hline${\text { POU } 5 F 1^{2} R}^{2}$ & 5'ctcctccgggttttgctccagct 3' & 58 \\
\hline MOG exon $2 \mathrm{~F}$ & 5'caggacagttcagagtgataggacc 3' & 60 \\
\hline MOG exon $2 \mathrm{R}$ & 5'attgctgcctcctcttggtaaga 3' & 60 \\
\hline Caja-G and E F & 5'gctcccactccatgaggtat 3' & 55 \\
\hline Caja-G & 5'tgagaggaggagagccta 3 ' & 55 \\
\hline Caja-E R & 5'cacgtgtcctccaggta 3' & 55 \\
\hline Klex exon 2-4on 2 consenso $\mathrm{F}$ & 5' gctcccactccatgaggtatt 3' & 53 \\
\hline Klex exon 2-4on 2 consensoR & $5^{\prime}$ cgcccacttctggaaggttc $3^{\prime}$ & 53 \\
\hline HLA B 326H24-1 A & 5'cttgtgtgccetccctcccc 3' & 52 \\
\hline HLA B 326H24-1 B & 5 'tgtttcctccccagtcatctttcct 3 & 52 \\
\hline HLA B 29717-4 A & 5'aagggacaagaacaatggaacagtgaa 3' & 52 \\
\hline HLA B 29717-4 B & 5'gctgtgtgtaagtggtgggggtg 3' & 52 \\
\hline HLA B 391L07-1 A & 5'taaagaaaggcaccaacaggattactatga 3' & 52 \\
\hline HLA B 391L07-1 B & 5'catgcttcctcctccacagttctacttt 3' & 52 \\
\hline G2482F ALU+470 & 5'ctgaaactggttctggttcatgtgac 3' & 53 \\
\hline G2483R ALU-166 & 5' gttagggatcgtttcctcag $3^{\prime}$ & 53 \\
\hline M13 rev & 5'ggaaacagctatgaccatga 3' & 50 \\
\hline M13 for & 5'ttgtaaaacgacggccagtg 3' & 50 \\
\hline RFB30-5' & 5' gctcagttctcctcaaaatgg 3' & 50 \\
\hline RFB30-3' & 5' gcccaaagaagggaatgacc 3' & 50 \\
\hline TCF19-5' & 5' gactttgctgccattaccatc 3' & 50 \\
\hline TCF19-3' & 5' gctcactctcatcatccagt $3^{\prime}$ & 50 \\
\hline TC4-5' & 5' gtccagttcaaacttgtattgt $3^{\prime}$ & 50 \\
\hline TC4-3' & 5' cacaggtcatcatcctcatc $3^{\prime}$ & 50 \\
\hline
\end{tabular}




\subsubsection{Hybridization probes for screening of CHORI BAC-bank and Southern blot and hybridization}

\begin{tabular}{|l|l|c|}
\hline Name of probe & \multicolumn{1}{|c|}{ Specification } & $\begin{array}{c}\text { Length of } \\
\text { fragment }\end{array}$ \\
\hline Caja G- class I & $\begin{array}{l}\text { Mini-prep, EcoRI digestion, loading for electrophoresis, } \\
\text { DNA extraction from gel }\end{array}$ & 750 \\
\hline K18T3 & $\begin{array}{l}\text { Mini-prep, EcoRI digestion, loading for electrophoresis, } \\
\text { DNA extraction from gel }\end{array}$ & 150 \\
\hline ATPV6g2 & $\begin{array}{l}\text { Mini-prep, EcoRI digestion, loading for electrophoresis, } \\
\text { DNA extraction from gel }\end{array}$ & 1300 \\
\hline BAT1 & $\begin{array}{l}\text { Mini-prep, BamHI and Hind III digestion, loading for } \\
\text { electrophoresis, DNA extraction from gel }\end{array}$ & \\
\hline TCF19 & $\begin{array}{l}\text { Mini-prep, Digestion BamHI and Hind III loading for } \\
\text { electrophoresis, DNA extraction from gel }\end{array}$ & 1000 \\
\hline HRC & $\begin{array}{l}\text { Mini-prep, EcoRI digestion, loading for electrophoresis, } \\
\text { DNA extraction from gel }\end{array}$ & 500 \\
\hline CAT 56 & $\begin{array}{l}\text { Mini-prep, EcoRI digestion, loading for electrophoresis, } \\
\text { DNA extraction from gel }\end{array}$ & $\begin{array}{l}\text { Mini-prep, EcoRI digestion, loading for electrophoresis, } \\
\text { DNA extraction from gel }\end{array}$ \\
\hline TRIM 39 & $\begin{array}{l}\text { Mini-prep, EcoRI digestion, loading for electrophoresis, } \\
\text { DNA extraction from gel }\end{array}$ & 550 \\
\hline TRIM 26 & $\begin{array}{l}\text { Mini-prep, EcoRI digestion, loading for electrophoresis, } \\
\text { DNA extraction from gel }\end{array}$ & 400 \\
\hline PPP1R11 & $\begin{array}{l}\text { Mini-prep, EcoRI digestion, loading for electrophoresis, } \\
\text { DNA extraction from gel }\end{array}$ & 500 \\
\hline TCTEX5 & $\begin{array}{l}\text { Mini-prep, EcoRI digestion, loading for electrophoresis, } \\
\text { DNA extraction from gel }\end{array}$ & $\begin{array}{l}\text { Mini-prep, EcoRI digestion, loading for electrophoresis, } \\
\text { DNA extraction from gel }\end{array}$ \\
\hline TCTEX4 & $\begin{array}{l}\text { PCR fragment } \\
\text { PCR fragment }\end{array}$ \\
\hline MOG & $\begin{array}{l}\text { HLA-B } \\
\text { ALU }\end{array}$ & 500 \\
\hline
\end{tabular}

3.7 DNA ladders and other standards for comparison of molecular size

\section{Standard samplel enzyme}

- Alkaline phosphatase (CIAP) $1000 \mathrm{U}$

- DNA from fish sperm

- DNA Ladder 100 bp Plus

- Gene Ruler DNA Ladder Plus100 bp

- Lambda DNA 500 bp

- Lamda DNA/ HindIII + EcoRI

- pUC 19 DNA 0,5mg/ml

- dNTP-Set 1
Supplier

Promega

Roche

Fermentas (MBI)

Fermentas

Fermentas

Fermentas

Fermentas

Roth
Ordering No.

M1821

10223646001

SM0322

SM0321

SD0011

SM0191

SD0061

K039.1 


\subsection{Vectors}

pDrive Quiagen

pTARBAC2.1 - BACPAC Resources Center

\subsection{BAC-clones used for genomic analysis}

To analyze MHC class I regions, clones from the BAC-bank library CHORI259 of the common marmoset were used. 42 selected clones were bought from BAC-PAC Resources Center at the Children's Hospital Oakland747 - 52 ${ }^{\text {th }}$ Street, Oakland CA 94609 USA, which is directed by Dr. Pieter deJong.

Table 3.9.1: Lab clone assignment to each GenBank BAC clone CHORI-259 of common marmoset.

The clones were named in lab clone 1 until clone 42, to practical reason. Each clone has the corresponds coordinate of GenBank BAC clone CHORI-259 of Common marmorset

\begin{tabular}{|l|c|}
\hline \multicolumn{1}{|c|}{ Clone } & Coordinates \\
\hline Clone 1 & $120 \mathrm{D} 3$ \\
\hline Clone 2 & $271 \mathrm{C} 5$ \\
\hline Clone 3 & $18 \mathrm{G} 14$ \\
\hline Clone 4 & $282 \mathrm{~L} 12$ \\
\hline Clone 5 & $204 \mathrm{C} 3$ \\
\hline Clone 6 & $127 \mathrm{E} 3$ \\
\hline Clone 7 & $239 \mathrm{~N} 18$ \\
\hline Clone 8 & $193 \mathrm{P} 12$ \\
\hline Clone 9 & $261 \mathrm{~L} 9$ \\
\hline Clone 10 & 282015 \\
\hline Clone 11 & $245 \mathrm{C} 6$ \\
\hline Clone 12 & $234 \mathrm{~L} 16$ \\
\hline Clone 13 & $463 \mathrm{N5}$ \\
\hline Clone 14 & $436 \mathrm{~B} 4$ \\
\hline Clone 15 & $19 \mathrm{I} 16$ \\
\hline Clone 16 & $44 \mathrm{G} 8$ \\
\hline Clone 17 & $510 \mathrm{~K} 19$ \\
\hline Clone 18 & $217 \mathrm{M} 17$ \\
\hline Clone 19 & $161 \mathrm{C} 12$ \\
\hline Clone 20 & $279 \mathrm{~K} 21$ \\
\hline Clone 21 & $325 \mathrm{P} 21$ \\
\hline Clone 22 & $277 \mathrm{~A} 2$ \\
\hline Clone 23 & $348 \mathrm{C} 21$ \\
\hline Clone 24 & $171 \mathrm{~K} 8$ \\
\hline
\end{tabular}




\begin{tabular}{|l|c|} 
Clone 25 & $174 \mathrm{~N} 1 \mathrm{a}$ \\
\hline Clone 26 & $224 \mathrm{H} 20$ \\
\hline Clone 27 & $347 \mathrm{~K} 7$ \\
\hline Clone 28 & $347 \mathrm{D} 1$ \\
\hline Clone 29 & $379 \mathrm{~F} 14$ \\
\hline Clone 30 & $425 \mathrm{~A} 13$ \\
\hline Clone 31 & $459 \mathrm{O} 20$ \\
\hline Clone 33 & $169 \mathrm{G} 2$ \\
\hline Clone 34 & $123 \mathrm{H} 17$ \\
\hline Clone 37 & $99 \mathrm{~L} 19$ \\
\hline Clone 38 & $334 \mathrm{C} 4$ \\
\hline Clone 39 & $329 \mathrm{M} 5$ \\
\hline Clone 40 & $379 \mathrm{M} 5$ \\
\hline Clone 41 & $174 \mathrm{~N} 1 \mathrm{~b}$ \\
\hline Clone 42 & $485 \mathrm{C} 4$ \\
\hline
\end{tabular}

Table 3.9.1 CHORI-259 BAC clone GenBank.

CHORI-259 Segment 1 High Density Filter set (4x4). Filters $01 \mathrm{I}-06 \mathrm{I}$, plate range between 0001 - 0288.

\begin{tabular}{|l|c|c|c|c|}
\cline { 2 - 6 } \multicolumn{1}{c|}{ Filter-Set } & \multicolumn{2}{c|}{ Manufacture dates } & \multicolumn{2}{c|}{ Library } \\
\cline { 2 - 6 } \multicolumn{1}{c|}{ 007874 } & $01 / 27 / 03-01 / 30 / 03$ & CHORI-259 & seg 1 \\
\hline Filter No. & Barcode & BAC-bank & Replica & Plate range \\
\hline 01I & 40819 & CHORI- 259 & R2 & 00010048 \\
\hline 02I & 40689 & CHORI- 259 & R2 & 00490096 \\
\hline 03I & 40713 & CHORI- 259 & R2 & 00970144 \\
\hline 04I & 40713 & CHORI- 259 & R2 & 01450192 \\
\hline $05 I$ & 40761 & CHORI- 259 & R2 & 01930240 \\
\hline 06I & 40785 & CHORI- 259 & R2 & 02410288 \\
\hline
\end{tabular}

Table 3.9.2 CHORI-259 BAC clone GenBank.

CHORI -259 Segment 2 High Density Filter set (4x4) Filters 06G- 011G, plate range between 0289 -.0528

\begin{tabular}{|c|c|c|c|c|c|c|}
\hline & \multicolumn{2}{|c|}{ Filter-Set } & \multicolumn{2}{|c|}{ Manufacture date } & \multicolumn{2}{|c|}{ Library } \\
\hline & \multicolumn{2}{|c|}{007897} & \multicolumn{2}{|c|}{$01 / 30 / 03-02 / 04 / 03$} & \multicolumn{2}{|c|}{ CHORI- 259 seg 2} \\
\hline \multicolumn{2}{|c|}{ Filter No. } & \multicolumn{2}{|c|}{ Barcode } & BAC-bank & Replica & Plate range \\
\hline \multicolumn{2}{|c|}{$07 \mathrm{G}$} & \multicolumn{2}{|c|}{40841} & CHORI- 259 & R2 & 02890336 \\
\hline \multicolumn{2}{|l|}{ 08G } & \multicolumn{2}{|c|}{40865} & CHORI- 259 & R2 & 03370384 \\
\hline \multicolumn{2}{|l|}{ 09G } & \multicolumn{2}{|c|}{40889} & CHORI- 259 & R2 & 03850432 \\
\hline \multicolumn{2}{|l|}{ 010G } & \multicolumn{2}{|c|}{40913} & CHORI- 259 & R2 & 04330480 \\
\hline \multicolumn{2}{|l|}{$011 G$} & \multicolumn{2}{|c|}{40937} & CHORI- 259 & R2 & 04810528 \\
\hline
\end{tabular}




\subsection{The gene sequence of Callitrix jacchus used as MHC class I probe}

The sequence was obtained and determined by Ulrike Geisler at the DPZ. The isolation of common marmoset MHC class I cDNA sequences from liver was carried out by reverse transcriptase polymerase chain reaction (RT-PCR). The primers were designed from primate consensus MHC class I sequences (Shufflebotham and Watkins, 1997; Cadavid et al, 1997; Cadavid et al, 1999). Sequence analysis of several clones indicated the presence of two different MHC class I gene, transcripts, eventually derived from a Caja-G and a Caja-E class I gene, respectively. She found one group of clones to be identical with the Caja-G*04 allele (GenBank accession number U59640), whereas the other group displayed $99 \%$ sequence identity with the Caja-E*02 allele (AF004920).

\subsection{Computer programs}

The DNA sequence was analyzed in internet with Program BLAST (Altschul et al., 1997, www.ncbi.nlm.nih.gov/blast. The program BLAST 2 SEQUENCE (www.ncbi.nlm.nih.gov/gorf/bl2.html) compared the similitude between two sequences. The DNA repeats in one sequence were searched by the program Repeat Masker (www.repeatmasker.org/cgi-bin/WEBRepeatMasker). For alignment analysis was used the programs BioEdit version 7.0.5 (Hall, 1999), Swissprot, AUGUSTUS and Genruner version 3.05. The construction Phylogenetic tree was made by the program Clustal $X$ (Version 1.83) and MEGA 3.1.

\subsection{Plastic articles}

\section{Article}

- Centrifuge tubes $13 \mathrm{ml}$ and $50 \mathrm{ml}$

- Combitips plus $5 \mathrm{ml}, 10 \mathrm{ml}$

- Cryo-tube vials $1.8 \mathrm{ml}$

- Electroporation cuvettes $2 \mathrm{~mm}$

- Non-skirted

- Nylon membrane 200 x 200

- Paper Whatman 3MM

- Petri box $\varnothing 9 \mathrm{~cm}$

- PCR containers $0.2 \mathrm{ml}$

- PCR plates 96 well, thermo fast

\section{Company}

Falcon

Eppendorf

Nunc

Peqlab

Abgene

Amersham

Schleicher and Schuell

Sarstedt

Eppendorf

Sarstedt 
- $96 \mathrm{~V}$ - soil microplates

- PP-tubes sterile, $15 \mathrm{ml}$

- PP-tubes, 170/77 $10 \mathrm{ml}$

- PP-test tubes, $50 \mathrm{ml}$

- Pump-spray bottle

- PE-spray bottle

- Safeseal tips, $2.5 \mu \mathrm{l}$

- Safeseal tips, $20 \mu \mathrm{l}$

- Safeseal tips, $100 \mu \mathrm{l}$

- Safeseal tips, $1000 \mu \mathrm{l}$

- Tips $2.5 \mathrm{ml}$

- Tips $20 \mathrm{ml}$, glass

\subsection{Radioactive substrate}

$\alpha^{32}$ P-dCTP250 $\mu$ Ci

\subsection{Lab equipment}

- Autoclave type A40/45

- Autoradiographic cassettes,

- Balance

- Developing machine, Kodak M35 X-OMAT processor,

- Centrifuge:

Mikro 22

Mikroliter 2025

Sigma 3k30,

Sepatech Varifuge 3.2 RS Heraeus

Labofuge GL

Minifuge $G L$

- $\mathrm{CO}_{2}$ Oven, B 5060,

- Electrophoresis camera (DNA)

- Electrophoresis power supply

- Electroporation- pulse generator
Biozym

Greiner

Greiner

Greiner

Roth

Neolab

Biozym

Biozym

Biozym

Biozym

Biozym

Biozym

Amersham

Webco

Dupont de Nemours

BP 3105 Sartorius

Kodak

Hettich

Hettich

Hettich

Heraeus

Heraeus

Heraeus

B1A, B2

Standard Power Pack P25 Biometra

EPI2500 
- Freezers $-20^{\circ} \mathrm{C}$ and $-80^{\circ} \mathrm{C}$

- Fume hood: Kojair

- Geiger counter

- Gel documentation

- Heating blocks thermomixer

- Hybridization oven Großer:

- Hybridization oven 6/12

- Incubator

- Incubator/shaker 3033

- Laminar flow cabinets,

- Light box Cronex-Kassetten,

- Magnetic stirrers MR 3001

- Microwave oven

- Parafilm

- PCR Machine

- Pipettes

- Power supplies

- $\mathrm{pH}$ meter

- Photometer GeneQuant pro,

- Refrigerators

- Sequence automat, ABI 3100-

- Speedvac evaporator

- UV Stratalinker 2400

- Vortex mixer

- Vortex

- Water baths

- Water purifiction equipment
Liebherr, Premium

BioFlow Technik,

Berthold LB 122

Jet Imager 2000, Intas

Eppendorf

Saur Laborbedarf

UNIEQUIP, UNITHERM

Heraeus

GFL

Heraeus

DuPont de Nemours

K. Heidolph

AEG Micromat

American National Can.

PCR System 2700 BioSystems

Eppendorf

Standard Power Pack P25 (Biometra)

Micropressor pH Meter, WTW, Schütt

Amersham Bioscience

Privileg

Avanta Genetic Analyzer

Applied Biosystems

Helmut Saur

Stratagene

L46 (GLW)

Genie 2, Scientific Industries

Schütt GFL 1083

Biocel MilliQ , Millipore Purification 


\subsection{Addresses of manufacturers}

- Amersham Bioscience, Munziger Str 9, 79111 Freiburg

- Applied Biosystems Applera, Frankfurter Str 12 b, 64293 Darmstadt

- Bachofer GmbH, Postfach 7089, 72770 Reutlingen

- Bauknecht Haushaltsgeräte $\mathrm{GmbH}$, Gottlob-Bauknecht Str.1-11, 73614 Schondorf

- Berthold Technologies \& Co KG, Calmbacher Str. 22, 75323 Bad Wildbach

- Biochrom AG, Postfach 460309, 12213 Berlin

- Biomol GmbH, Waidmann Str. 35, 22769 Hamburg

- Biozym Scientific GmbH, Postfach 63, 31833 Hessisch Oldendorf

- Calbiochem-Novobiochem GmbH, Postfach 116, Bad Soden

- Carl-Roth GmbH \& CoKG, Schoemperlen Str. 1 - 5, 76185 Karlsruhe

- Dupont de Nemours GmbH, Sägewerk Str. 3, 83395 Freilassing

- Eppendorf AG, Friedens Str. 116, 51145 Köln

- Fischer, Dr.K, Schneidemühl Str. 9, 69115 Heidelberg

- Fluka Chemie AG, Industrie Str. 25 CH-9471 Buchs

- Fröbel Laborgeräte, Alwinder Str. 4, 88131 Lindau

- GeneCraft GmbH, Reiffeisten Str. 12, 59348 Lüdinghausen

- Genomed GmbH, Wieland Str. 28a, 32545 Bad Oeynhausen

- Gilson International Deutschland, Otto-Hahn Str. 17, 65520 Camberg

- Hereus, Kendro Laboratory Products GmbH, 63450 Hanau

- Helmut Saur Laborbedarf, Carl-Zeiss Str. 58, 72770 Reutlingen

- Hettich GmbH \& Co KG, Industrie Str. 2 10, 31311 Uetze/ Hänigsen

- Hewlett-Packard GmbH, Postfach 1430, 71004 Böblingen

- Intas Science Imaging Instruments GmbH, Florenz-Sartorius Str. 14, 37079 Göttingen

- Invitrogen $\mathrm{GmbH}$, Technologiepark Karsruhe, Emmy-Noether Str. 10, 761331 Karlsruhe

- Janke \& Kunkel GmbH \& Co KG, Janke \& Kunkel Str. 10, 79219 Stauffen

- Köttermann GmbH \& Co KG, Industrie Str. 2-10, 31311 Uetze/Hänigsen

- Küjner, Adolf, Dinkelberg Str. 1, CH-4172 Birsfelden

- Laboratorium Prof. Dr. Berthold, Siemens Str. 4, 30173 Hannover

- MBI Fermentas GmbH, Opel Str. 9, 68789 St. Leon-Rot 
- Membrane Pure GmbH, Am Kuemmerling Str 37, 55294 Bodenheim

- Merck KGaA, Frankfurter Str. 250, 64293 Darmstadt

- MWG Biotech AG., Azinger Str. 7a, 85560 Ebersberg

- NEB, New England Biolabs GmbH, Brüning Str. 50, 5926 Frankfurt am Main

- Peqlab Biotechnologie GmbH, Carl-Thiersch Str. 2b, 91052 Erlangen

- Polaroid GmbH, Sprendlinger Lands Str. 109, 63069 Offenbach

- Promega GmbH, Schildkröt Str. 15, 68199 Mannheim

- Quiagen GmbH Max-Volmer Str. 4, 407024 Hilden

- Renner GmbH, Ried Str. 6, 67125 Darmstadt

- Roche Diagnostic GmbH, Sandhofer Str. 116, 68305 Mannheim

- Thermo Haake, Diesel Str.4, 76227 Karlsruhe

- W. Krannich GmbH \& Co KG, Elliehäuserweg Str17, 37079 Göttingen

- Sarstedt AG \& Co, Postfach 1220, 51582 Nümbrecht

- Sartorius AG, Weender Lands Str. 94-108, 37075 Göttingen

- Schleicher \& Schuell BioScience GmbH, Postfach 1111, 38582 Dassel

- Schott-Geräte-GmbH, Hattenberg Str.10, 55122 Mainz

- Schütt Labortechnik GmbH, Rudolf-Wissel Str. 11, 37079 Göttingen

- Serva Electrophoresis GmbH, Carl-Benz Str. 7, 69115 Heidelberg

- Sigma-Aldrich Chemie GmbH, Eschen Str. 5, 82024 Taufkirchen

- Sorvall, Kendro Laboratory Products GmbH, Robert-Bosch Str.1, 63505 Langenselbold

- Vesper GmbH, Industrie Str. 16, 49079 Göttingen

- Webeco GmbH \& Co KG, Mühlen Str. 38, 23611 Bad Schwartau

- Whatman Biometra GmbH, Rudolf-Wissel Str. 30, 37079 Göttingen 


\section{Methods}

\subsection{Preparation of materials}

The preparation of buffers, reagents and plastic materials used in the manipulation of nucleic acids was as follows: For all stock solutions, water was used which had been both deionized and bidistilled. All reagents were of the highest grade available.

The sterilization of materials was performed either by

- filtration through a $0.22 \mu \mathrm{m}$ filter or by

- autoclaving of solutions for $15 \mathrm{~min}(\mathrm{~min})$ at $121^{\circ} \mathrm{C}=15$ bars.

There are toxic, carcinogenic, mutagenic and teratogenic reagents. For this reason, the user must proceed with precaution; the protective equipment includes eye protection, laboratory coat and gloves.

For the use of radioactive isotopes it is very important to know the applicable regulations and approved procedures. The levels of radioactivity e.g. from ${ }^{32} \mathrm{P}$ in the working area should be checked with a hand-held Geiger mini-monitor, both near the containers and in the working area.

The strength of radioactivity in a source is defined by an international SI unit, the Becquerel $(\mathrm{Bq})$.

1 Becquerel $(\mathrm{Bq})=1$ disintegration per second (e.g within a radioactive specimen).

Definitions in Curie ( $\mathrm{Ci}$ ) may be converted into $\mathrm{Bq}$ or disintegrations per min (dpm) by the equations

$1 \mathrm{Ci}=3.7 \times 10^{10} \mathrm{~Bq}$;

$1 \mathrm{Ci}=2.2 \times 10^{12}(\mathrm{dpm})$;

1 microcurie $(\mu \mathrm{Ci})=3.7 \times 10^{4} \mathrm{~Bq}=2.22 \times 10^{6} \mathrm{dpm}$.

The relative centrifugal force (RFC) is measured in $x g$, it is used to specify forces applied in centrifugation. It is proportional to the speed in rpm (revolutions per min) of a centrifuge and depends on the rotor model (with the maximum spinning radius of tubes therein).

The relationship between RCF and speed (rpm) is determined by the following equation: $\mathrm{RCF}=11.12 r(\mathrm{rpm} / 1000)^{2}$

where $r$ is the radius between the rotating particle and the axis of rotation. 


\subsection{Filters of carrying bacterial artificial chromosome (BAC) clones for screening}

\subsubsection{Source and origin of BAC bank CHORI of common marmoset (Callithrix} jacchus)

The BACPAC Resource Center (BPRC) at Children's Hospital Oakland Research Institute (CHORI) in Oakland, California (United States) has prepared BAC and hybridization (PAC) genomic DNA libraries and provides copies thereof with highdensity colony hybridization filters.

In Pieter De Jong's Laboratory at the Children's Hospital Oakland Research Institute, BACPAC Resources Center, a BAC library CHORI-259 of common marmoset (Callithrix jacchus, male) was constructed by Doriana Misceo and Dr. Baoli Zhu using the cloning techniques developed in the same laboratory (Osoegawa et al., 1998). Dr. Suzette Tardif, Associate Director of the Southwest National Primate Research Center in San Antonio, TX provided the marmoset sample to make the library. Cells were taken from the kidney of a male marmoset (SNPRC \#17081). The DNA was isolated and then partially digested with a combination of ECoRI restriction enzyme and EcoRI methylase. The DNA fragments were ligated into the pTARBAC2.1 vector between the EcoRI sites. The ligation products were transformed into DH10B (T1 resistant) electrocompetent cells (Invitrogen, 1999).

4.2.2 Organization of BAC bank CHORI 259 from of the new world monkey common marmoset (Callithrix jacchus) on microtiter plates and on nylon filters The whole library was arrayed into 528 384-well microtiter plates and gridded also onto eleven nylon filters high-density $(22 \times 22 \mathrm{~cm})$ as tools for screening by probe hybridization. Each hybridization membrane represents over 18.000 distinct BAC clones, stamped in duplicate using a code of arrangements. It indicates as amend event supplementing the two coordinate numbers up to the full bank number of the clone.

\subsubsection{Procedure for hybridization on high density filters}

The filters were soaked in pre-hybridization solution and then placed in a bottle; $20 \mathrm{ml}$ of the pre-hybridization solution were added to pre-hybridize for $30 \mathrm{~min}$ at $60{ }^{\circ} \mathrm{C}$. Thereafter, a labeled probe was added to hybridize overnight (16-18 hours) at $60{ }^{\circ} \mathrm{C}$. The filters were washed with wash I solution at $60{ }^{\circ} \mathrm{C}$ for $30 \mathrm{~min}$ and this step was repeated twice. 
The filters were wrapped individually in plastic wrap and placed in a large cassette (35 $\mathrm{x}$ $43 \mathrm{~cm}^{2}$ ), two in each cassette. A light overlapping of the filters occurred, but this did not give rise to carry problems in the reading of data, see figure 4.1. For the choice of sensitivity, the exposition for autoradiography was done for 2 to 36 hours at $-80^{\circ} \mathrm{C}$. The position of the positive clones was viewed by the use of a transparent net (overlay template, see Figure 4.2) which allows definition of the coordinates (as an orientation guide for the choice) of $t$ he interesting clones.

The filters were stripped off the differently labeled DNA probes by boiling them in $0.1 \%$ SDS for 15 min. Stripped filters were preserved in plastic wrap at $-20{ }^{\circ} \mathrm{C}$.

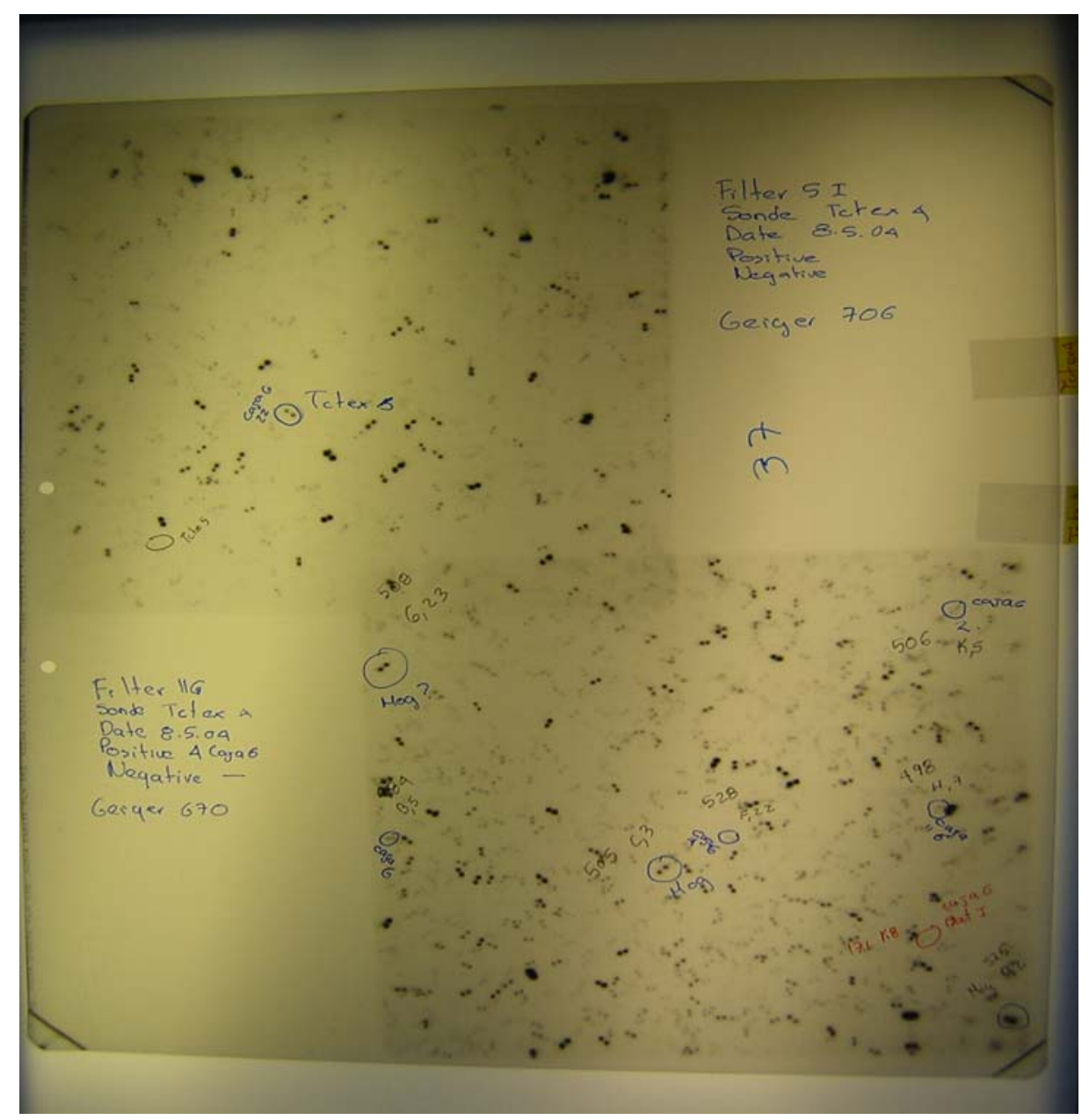

Figure 4.1: Example of placing two BAC bank filters in one cassette for autoradiography. The BAC clone filter $10 \mathrm{G}$ and $11 \mathrm{G}$ were hybridized with TCTEX4 probe the centre, a small overlap of screened filters is seen, this presented no problem in the reading of the data, and the two points indicated the double positive control for each clone. 


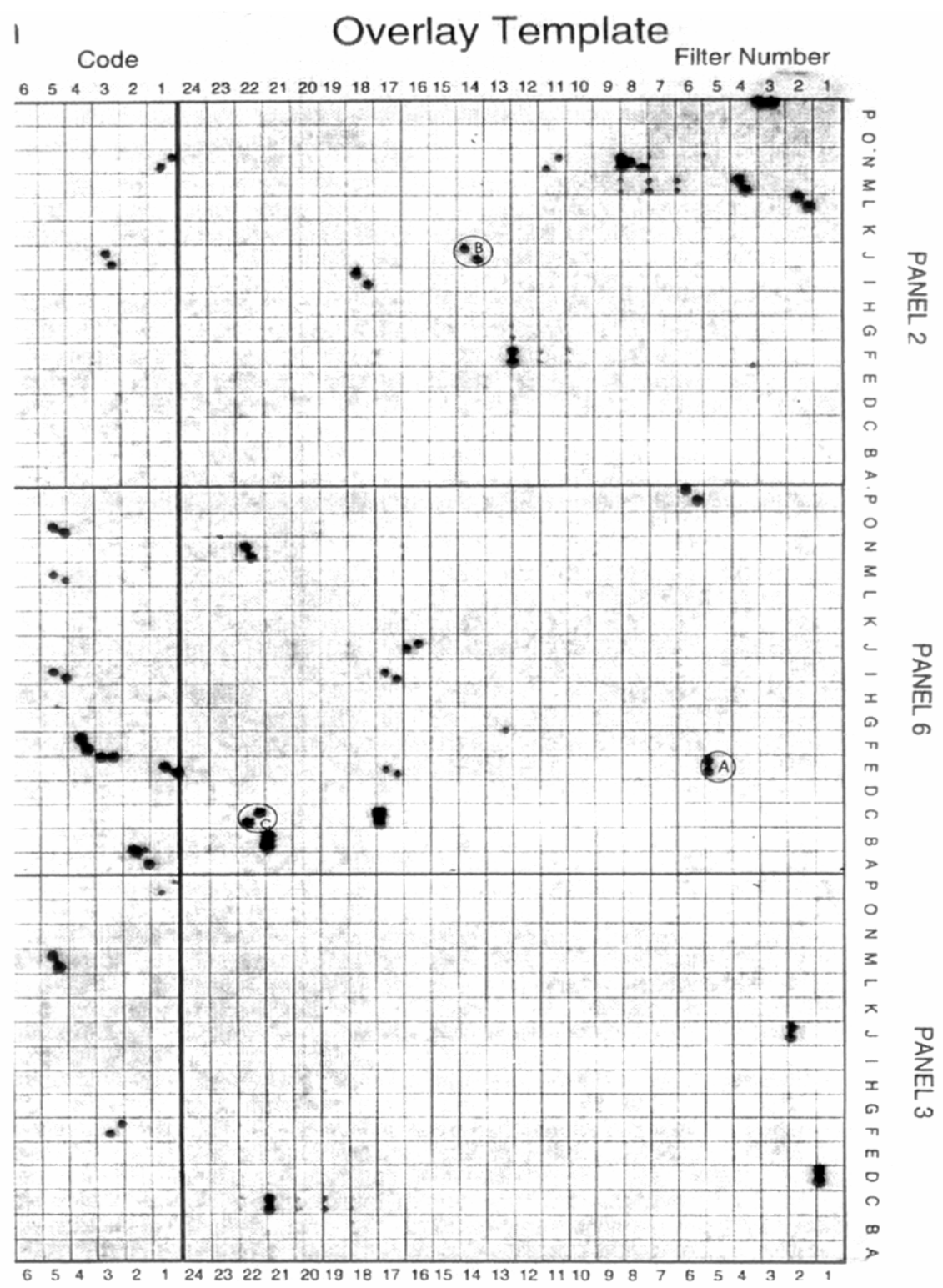

Figure 4.2: Example of a positive clone localization in originals 384 well plates. Here, the overlay template is placed over the autoradiography identifying clones positive in screening with the hybridization probe (http://bacpac.chori.org).

\subsection{Preparation of a BAC clone cultures}

Each clone was received as a bacterial LB agar stab culture. The DH10 E.coli host had been placed into LB agar containing $12.5 \mu \mathrm{g} / \mathrm{ml}$ chloramphenicol for BAC clones. The DH10 E.coli host harbors a number of identical plasmids consisting of vector insert DNA BAC clone (Figure 4.3). This culture has a finite life time at $4{ }^{\circ} \mathrm{C}$.

To inoculate a single isolated bacterial colony from the stab end fare received into $2 \mathrm{ml}$ LB medium supplemented with $20 \mu \mathrm{g} / \mathrm{ml}$ chloramphenicol in a sterile toothpick was used. The culture was grown for 3 hours by shaking at $5-6 \mathrm{~g}$ at $37^{\circ} \mathrm{C}$. This $2 \mathrm{ml}$ culture 
was transferred into a $50 \mathrm{ml}$ snap-cap polypropylene tube with $10 \mathrm{ml}$ LB medium supplemented with chloramphenicol and grown overnight (up to 16 h) by shaking at 5 $6 \mathrm{~g}$ at $37^{\circ} \mathrm{C}$.

$1 \mathrm{ml}$ of glycerol stocks of cells was prepared as follows: $1 \mathrm{ml}$ of a freshly grown overnight culture was supplemented with $40 \%$ of sterile glycerol, mixed well, transferred to two freezer vials and immediately placed into a dry ice/ethanol bath or into a box in the $-80^{\circ} \mathrm{C}$ freezer.

\subsection{Isolating plasmid DNA (Miniprep)}

This is a rapid alkaline lysis miniprep method. The culture was centrifuged at $400 \mathrm{~g}$ for $10 \mathrm{~min}$. The supernatant was discarded, the pellet resuspended in $0.3 \mathrm{ml} \mathrm{P1}$ solution plus $0.3 \mathrm{ml}$ of P2 solution by shaking the tube gently to mix contents. For hydrolysis, it was left at room temperature for $5 \mathrm{~min}$. Thereafter, $0.3 \mathrm{ml}$ of P3 solution was added slowly, the tubes shaken being gently during addition. After having been placed in ice for at least $5 \mathrm{~min}$, they were centrifuged at $5.000 \mathrm{~g}$ for $15 \mathrm{~min}$ at $4{ }^{\circ} \mathrm{C}$. Afterwards, the supernatant was collected using a pipette; this extraction step was repeated three times, then, $2 \mathrm{ml}$ of ice-cold isopropanol was added to the combined supernatants any white precipitating material being avoided. The tubes were mixed by inverting a few times, placed on ice for at least 5 min and centrifuged with maximum velocity in a cold microcentrifuge for $15 \mathrm{~min}$. The supernatant was discarded and $0.5 \mathrm{ml}$ of $70 \%$ Etanol was added to wash the DNA pellets by inverting them several times. Thereafter, they were collected by centrifugation in a cold microcentrifuge at $10.000 \mathrm{~g}$ for $5 \mathrm{~min}$. The supernatant was removed as much as possible. Occasionally, pellets become dislodged from the tube therefore, it is better to carefully aspirate off the supernatant rather than to pour it off. The DNA pellets were dried at room temperature and resuspended in $40 \mu \mathrm{l}$ of $\mathrm{H}_{2} \mathrm{O}$ with shaking. 


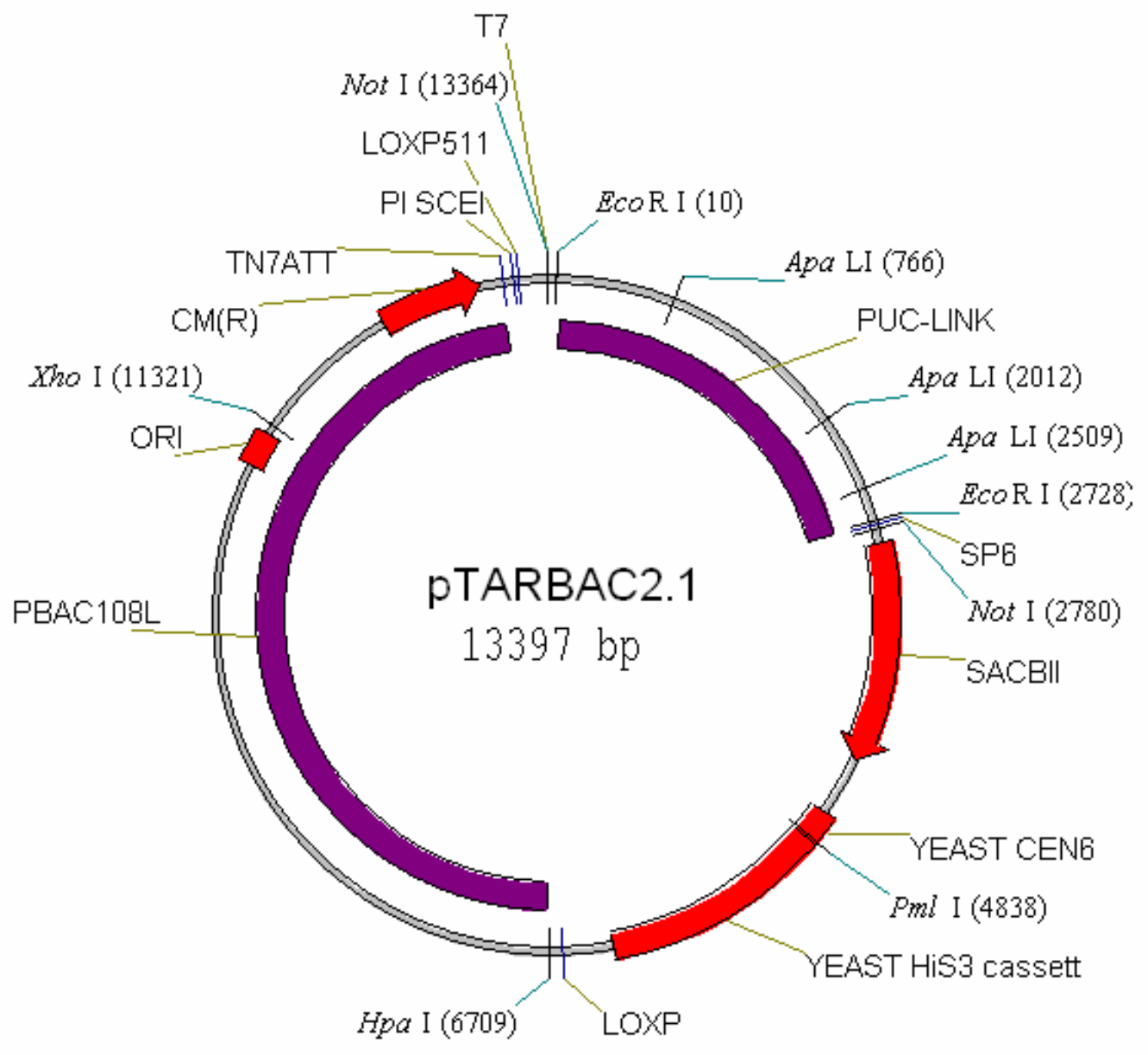

Figure 4.3: Map of the plasmid pTARBAC 2.1 (vector) (http://bacpac.chori.org/ptar bac21.htm).

This is the cloning vector of CHORI 259, the BAC bank of the common marmoset. It contains, among many others, a gene encoding resistance to chloramphenicol and shows various restriction enzymes, for example EcoRI, where to cut a DNA fragment.

\subsection{Quantification of DNA}

\subsubsection{UV-absorption spectroscopy method}

The concentration of a DNA solution is estimated by measuring its absorption at several wavelengths (260 nm, $280 \mathrm{~nm}$ and $320 \mathrm{~nm}$ ) which also provides an indicator of the purity of the preparation. The absorption spectrum of DNA shows a maximum at 260 $\mathrm{nm}$. In the case of double-stranded DNA one $A_{260}$ or $\left(O D_{260}\right)$ unit (measured by a light path of $1 \mathrm{~cm}$ ) corresponds to a concentration of $50 \mu \mathrm{g} / \mathrm{ml}$.

Significant absorption at $230 \mathrm{~nm}$ indicates contamination by phenolate ions and other organic compounds (Stulnig and Amberger, 1994), elevated absorption at $280 \mathrm{~nm}$ 
indicates the presence of protein, whereas absorption at $320 \mathrm{~nm}$ indicates the presence of particulate matter. In general, highly pure DNA has an $A_{260} / A_{280}$ ratio > 1.8.

The DNA was dissolved in water, and water was used as a blank or reference sample. The DNA concentrations were calculated using the following equation:

Sample DNA concentration

$\mathrm{C}=\mathrm{A}_{260} \bullet 50 \mu \mathrm{g} \mathrm{DNA} / \mathrm{ml} \bullet$ dilution vol/sample vol $\bullet \mathrm{cm}$ path ${ }^{-1}(\mu \mathrm{g} \mathrm{DNA} / \mathrm{ml}) ;$ light path (cm)

$A_{260}=A_{260}$ corrected for turbidity

$50 \mu \mathrm{g}$ DNA/ml = DNA conc. specific absorption factor

dilution vol/sample vol $\bullet=$ dilution factor

in short $\mathrm{C}=\mathrm{A}_{260}$ corrected $\bullet 50 \mu \mathrm{g} \mathrm{DNA} / \mathrm{ml} \bullet \mathrm{V} \bullet \mathrm{f}^{-1}(\mu \mathrm{g} \mathrm{DNA} / \mathrm{ml})$

$\mathrm{C}=$ concentration $(\mu \mathrm{g} / \mathrm{ml})$,

$A_{260}=U V$ absorption at wave length $260 \mathrm{~nm}$

$\mathrm{V}=$ dilution factor = dilution volume/sample volume;

$\mathrm{f}=$ cuvette length (light path) in $\mathrm{cm}$ (can be neglected, $1 \mathrm{~cm}$ - cuvettes were used.

\subsubsection{Minigel method}

This is a rapid and convenient method to measure the quantity of DNA and to analyze its intactness. Into a slot of a $0.8 \%$ agarose minigel containing ethidium bromide (0.5 $\mu \mathrm{g} / \mathrm{ml}) 2 \mu \mathrm{l}$ of the DNA solution, mixed with $1 \mu \mathrm{l}$ gel loading buffer $6 \mathrm{x}$ and $3 \mu \mathrm{l}$ water was applied. As DNA standards, two samples of plasmid DNA pUC19 vector $(25 \mu \mathrm{g} / \mu \mathrm{l}$ and $50 \mu \mathrm{g} / \mu \mathrm{l}$ ) were run in parallel. Electrophoresis was carried out for $20 \mathrm{~min}$ at 100 volts in TBE $1 X$ buffer. The gel shown in Figure 4.4 was photographed using a length of wave length UV irradiation of $260 \mathrm{~nm}$. 


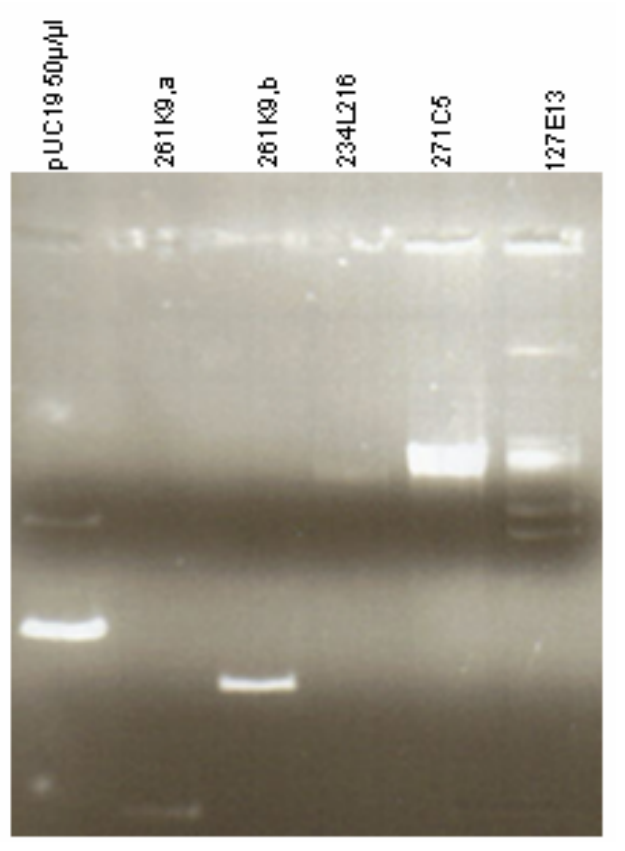

Figure 4.4: Quantitative staining of DNA an agarose gel containing ethidium bromide.

Image of a $1 \%$ agarose gel stained with $20 \mathrm{mg} / \mathrm{ml}$ ethidium bromide. DNA fragment extraction from single bands performed with $\mathrm{Nal}$ as described in the text. pUC19 $50 \mu / \mu \mathrm{l}$ was the probe control to quantification of DNA by comparation.

\subsection{Restriction digestion of plasmid DNA}

The treatment of plasmid DNA with a restriction endonuclease produces a series of precisely defined fragments which are separated according to size by gel electrophoresis.

1 unit $(U)$ of restriction enzyme is defined as the amount of enzyme required to digest 1 $\mu \mathrm{g}$ of lambda DNA in 1 hour at $37^{\circ} \mathrm{C}$ in $50 \mu$ of assay buffer.

The digestion protocol of DNA requires in a $20 \mu \mathrm{l}$ volume of final reaction mixture $2 \mu \mathrm{g}$ DNA for every $2 U$ of enzyme, the adequate amount of buffer, and for some enzymes $10 \mathrm{mg} / \mathrm{ml} \mathrm{BSA}$. The mixture is incubated at $37^{\circ} \mathrm{C}$ the incubation time depends on the enzyme, ranging between $1.5-3$ hours. The enzyme is inactivated at $65^{\circ} \mathrm{C}$, figure 4.5 . 


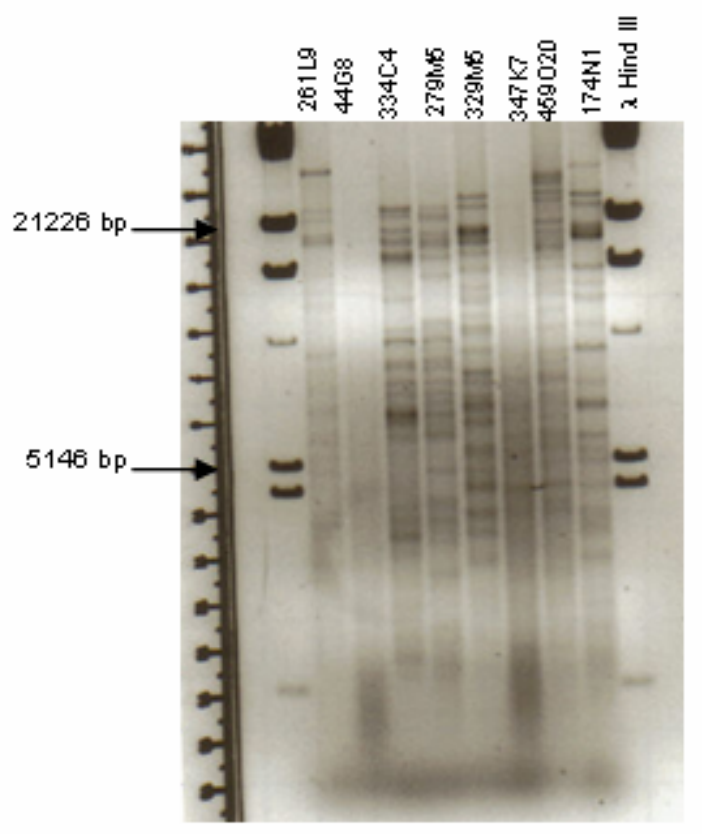

Figure 4.5: Fragments obtained by restriction digestion separated by agarose gel electrophoresis.

The treatment of DNA plasmid clones 9 (261L9), 16 (44G8), 38 (334C4), 40 (379M5), 39 (329M5), 27 (347K7), 31 (459O2) and 41 (174N1) with restriction endonuclease EcoRI and lambda vector digested Hind III produced a series of precisely defined fragments. These were separated according to size by electrophoresis in $0.8 \%$ agarose gel containing $20 \mathrm{mg} / \mathrm{ml}$ ethidium bromide. The figure shows DNA by its UV absorption. Note: the picture is a negative image.

\subsection{Separation of large DNA fragments by agarose gel electrophoresis}

Agarose gel electrophoresis is an effective standard method for separating, identifying and purifying DNA fragments in the range of 0.5 to $25 \mathrm{~kb}$. The gel is prepared with an appropriate agarose concentration. Voltage and running time are chosen for optimal separation. The DNA and its fragments, stained by ethidium bromide contained in the gel, are visualized directly upon illumination with UV light. DNA molecules exposed to an electric field (defined by length of gel and a potential difference at its ends) migrate towards the anode due to the negatively charged phosphate groups along the DNA backbone.

The gels were prepared with an adequate volume of electrophoresis buffer (TBE or TPE 1X) with the desired amount of electrophoresis-grade agarose to a volume of electrophoresis buffer sufficient for constructing the gel. The agarose was melted in a 
microwave oven at $55{ }^{\circ} \mathrm{C}$. For fingerprints and DNA concentration gel it typically contained $0.8 \%$, or for the other purposes $1.5 \%$. Generally a $20 \mu \mathrm{g} / \mathrm{ml}$ ethidium bromide solution was added so that a final concentration of $0.5 \mu \mathrm{g} / \mathrm{ml}$ was obtained.

After the gel had hardened, it was placed into an electrophoresis chamber, with sufficient electrophoresis buffer to cover the gel. The DNA sample was prepared with 6x loading buffer. The samples were run in parallel with a DNA ladder or other DNA as molecular size standard.

The progress of separation at 50 - 120 volts within a limiting time was monitored by vision using UV light to illuminate the migrating stained DNA (see Figure 4.5).

\subsubsection{Fluorescence photography of DNA in agarose gels}

The DNA in agarose gels stained with ethidium bromide was photographed by illumination with UV light $\left(>2500 \mu \mathrm{W} / \mathrm{cm}^{2}\right)$ in a complete photographic documentation system (UV transilluminator with a Polaroid camera and a digital photograph system), see figure 4.6 .

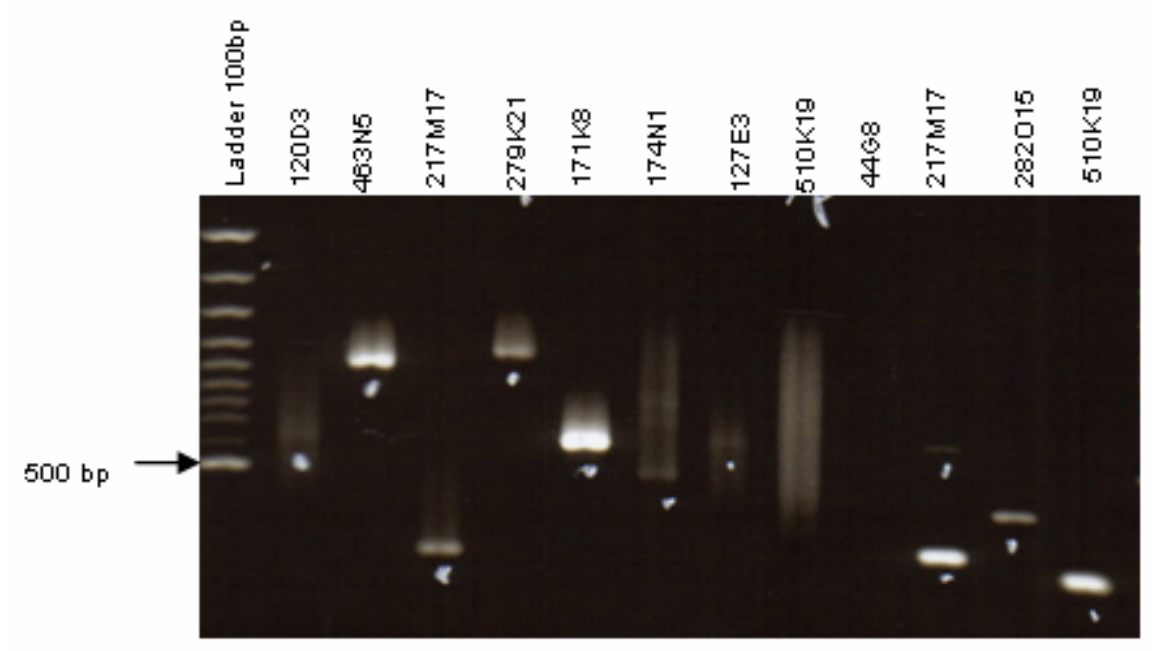

Figure 4.6: Fluorescence photograph of a $1 \%$ agarose gel.

After electrophoresis of various DNA samples from BAC clones obtained by PCR with primer pTARBAC 3 in a $1 \%$ agarose gel stained with $20 \mathrm{mg} / \mathrm{ml}$ ethidium bromide; ladder marker sizes 100 bp.

\subsection{Extraction of DNA fragments from agarose gels}

This method was used for different purposes:

- To isolate fragments of the inserts from different plasmids containing probes, e.g. framework genes, MHC class I Caja-G gene and others; 
- to isolate fragments as hybridization probes;

- to isolate fragments after inverse PCR for sequencing.

The segment of a gel containing the DNA fragment of interest is excised (approximately $100 \mathrm{mg}$ ) and incubated with $6 \mathrm{M}$ sodium iodide solution (Nal) at $55^{\circ} \mathrm{C}$ until the agarose has melted (Figure $4.7 \mathrm{a}$ and $4.7 \mathrm{~b}$ ). Thereafter, $20 \mu \mathrm{l}$ of silica gel are added and the sample is incubated at $55^{\circ} \mathrm{C}$ for $10 \mathrm{~min}$. It is centrifuged for one min at $21.000 \mathrm{rpm}$ and the supernatant discarded.

The pellet is washed with $300 \mu \mathrm{l}$ of the Nal solution and, thereafter, with $70 \%$ ethanol. It is dried, resuspended in $20 \mu \mathrm{l}$ of water and incubated for $20 \mathrm{~min}$. Thereafter, the mixture is centrifuged at maximum speed for one min.

The supernatant contains the DNA, which is transferred in a new tube and the concentration and quality of the DNA is checked by agarose gel electrophoresis; see Figure 4.7c.

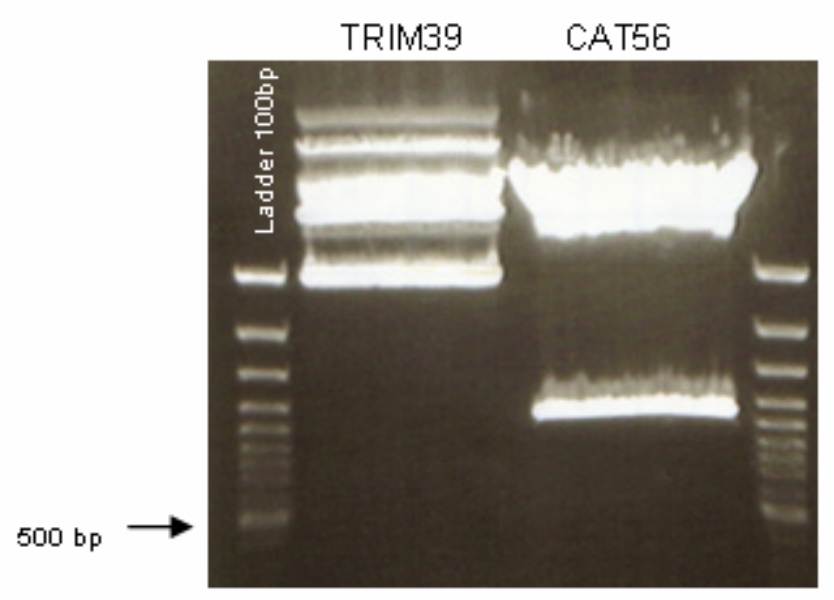

Figure 4.7 a: Electrophoretic separation of fragments after restriction digestion of two clones with TRIM 39 and CAT56 framework genes, respectively.

Two clones with TRIM 39 and CAT 56 framework genes were digested with restriction endonucleases $\mathrm{BamHI}$ and HindIII. These were separated according to size by electrophoresis in $0.8 \%$ agarose gel containing $20 \mathrm{mg} / \mathrm{ml}$ ethidium bromide, ladder marker sizes $100 \mathrm{bp}$. The figure shows DNA by its UV absorption. 


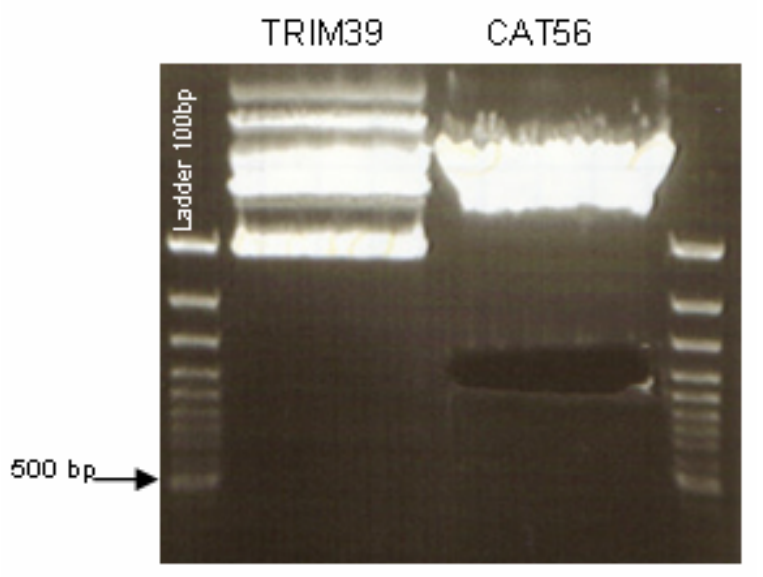

Figure 4.7 b: Cutting a fragment from a clone containing the CAT56 framework gene.

The $950 \mathrm{bp}$ fragment of this clone with the CAT 56 framework gene was separated by gel electrophoresis in $0.8 \%$ agarose gel containing $20 \mathrm{mg} / \mathrm{ml}$ ethidium bromide, ladder marker sizes $100 \mathrm{bp}$. Then excised for extraction of DNA from the gel sample by Nal method.

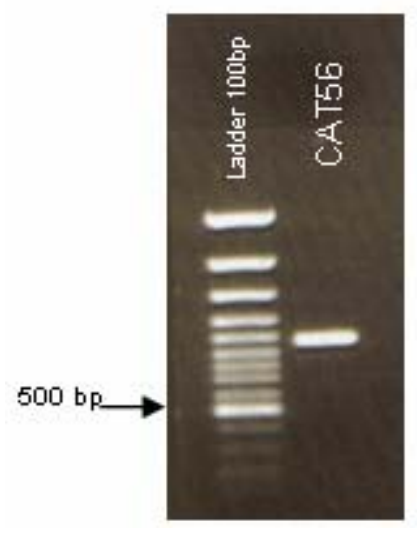

Figure $4.7 \mathrm{c}$ : Agarose gel electropherogram for control of intactness of a fragment, CAT56.

The DNA gel extraction was done with $\mathrm{Nal}$, and then was checked by electrophoresis in $1 \%$ agarose gel stained with $20 \mathrm{mg} / \mathrm{ml}$ ethidium bromide, ladder marker sizes $100 \mathrm{bp}$. 


\subsection{Random primer method}

Various specifically labeled oligonucleotide primers can assist by DNA polymerase in the initiation of DNA synthesis on single-stranded templates. Products of this primed DNA synthesis are radiolabeled by the use of one $\left[\alpha^{-32} \mathrm{P}\right] \mathrm{dCTP}$ and three unlabeled dNTP's as substrates.

Random priming, i.e. with a commercially available mixture of different primers was employed to label probes for screening of BAC clones and for analyzing plasmid DNA by hybridization after blotting. The probes were obtained by digestion of clones containing the fragments of interest. The DNA fragment was extracted from an agarose gel after electrophoresis.

For random priming, the commercial kit Megaprime DNA labeling system from Amersham or Promega was used. The protocol for the polymerase reaction is as follows: First, $25 \mathrm{ng}$ of template DNA, $5 \mu \mathrm{l}$ of $10 \mathrm{mM}$ primer and $30 \mu \mathrm{l}$ of bidistilled water are incubated at $100^{\circ} \mathrm{C}$ for $5 \mathrm{~min}$. After the tube is on ice, the following reagents are added to a final reaction volume of $50 \mu \mathrm{l}: 10 \mu \mathrm{l}$ of reaction buffer, $0.5 \mu \mathrm{l}$ of $2 \mathrm{mM}$ dNTPs $(A, G, T), 2 \mu l$ of Klenow-DNA-polymerase and $50 \mu \mathrm{Ci}\left[\alpha^{32} \mathrm{P}\right] \mathrm{dCTP}$. The mixture was incubated at $37^{\circ} \mathrm{C}$ for $30 \mathrm{~min}$, when the Amersham kit was used, and for one hour at room temperature when the Promega kit was used. The labeled probe was denatured at $100^{\circ} \mathrm{C}$ for $5 \mathrm{~min}$ and, after being chilled could be added to the hybridization solution. Labelled probes were stored at $4^{\circ} \mathrm{C}$ (Feinberg and Vogelstein, 1983).

\subsection{Analysis of plasmid DNA by blotting and hybridization}

\subsubsection{Southern blotting}

Southern blotting is the transfer of DNA fragments from an electrophoresis gel to a nylon membrane support. DNA fragments are immobilized as result of the transfer and of subsequent treatment. The membrane thus contains a semi-permanent reproduction of the banding pattern of the gel.

The gel containing the EcoRI digested plasmid DNA for every clone and the appropriate DNA size marker (100 bp) was stained with ethidium bromide and blotted onto a nylon membrane, positively charged by its chemical nature. To identify the positions of bands in the gel, it was photographed with a ruler laid along its side (Figure 4.8). The results obtained by this methodology are used for sequencing and mapping genes.

The protocol is divided into three stages: 1 . preparation of the gel, 2 . set up of the transfer and 3. immobilization of DNA. 


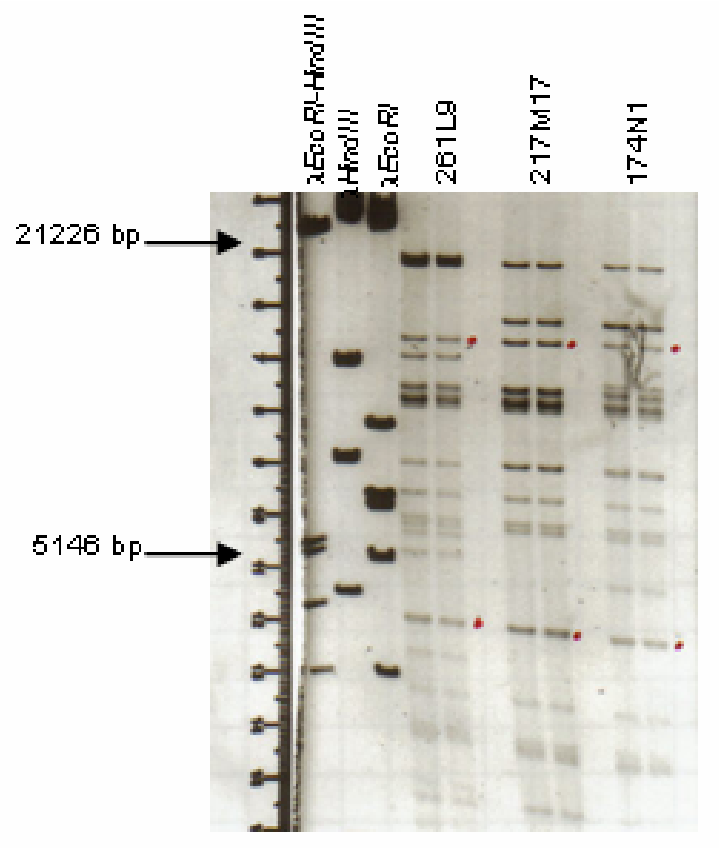

Figure 4.8: A gel before the Southern blotting.

In this $0.8 \%$ agarose gel containing ethidium bromide, fragments from the clones 9(261L9), 18(217M17) and 25(174N1) have been subject to electophoresis, size markers, lambda DNA digested with EcoRI, EcoRI-HindIII and Hind III, respectively. On the left is a ruler to identify the fragments independently of any size changes of the picture. Note: the picture is a negative image.

\subsubsection{Preparation of the gel}

The agarose gel was pretreated by soaking in a series of solutions which depurinate, denature and neutralize the DNA.

The gel was rinsed in distilled water for $30 \mathrm{~min}$ on a platform shaker with slow agitation at room temperature. The water was discarded and the gel placed in 10 gel volumes of $0.25 \mathrm{M} \mathrm{HCl}$ to result in a partial depurination of the DNA fragments.

The $\mathrm{HCl}$ was poured off and the gel rinsed in distilled water. Finally 10 gel volumes of denaturation solution were added, and agitation continued for $20 \mathrm{~min}$.

The denaturation solution was poured off and the gel rinsed with distilled water.10 volumes of neutralization solution were added, and agitation continued for $20 \mathrm{~min}$. At this stage, the gel was ready to start its transfer onto the nylon membrane. 


\subsubsection{Set up of the transfer}

The gel was placed on the plane surface of a reservoir and submerged half with transfer buffer 20x SSC.

A piece of nylon membrane just large enough to cover the exposed surface of the gel, was cut, laid into the gel and squeezed to disperse air bubbles by rolling over it a glass pipette over the surface.

Several pieces of Whatman 3M filter paper were put over the nylon membrane. Finally, a glass plate was laid on top of the pile, and a weight $(0.2-0.4 \mathrm{~kg})$ was placed on top to hold everything in place. The transfer was overnight.

\subsubsection{Immobilization of the DNA}

Before immobilization of DNA, the filter paper was removed and the membrane recovered. Beforehand, the position of the wells on the membrane was marked with a soft pencil to ensure that the up-down and back-front orientations remained recognizable.

When the membrane was air dried, the cross-linking was achieved by UV ligth for 30 sec at $1200 \mu \mathrm{J}$. This leads to covalent attachment of DNA and enables the membrane to be reprobed several times.

\subsubsection{Hybridization analysis of DNA}

Hybridization analysis is sensitive and permits detection of even a single copy of a gene within a complex genome. The principle of this hybridization analysis is that single stranded DNA molecules of defined sequence (the probe) can base pair to a second DNA molecule that contains a complementary sequence (the target).

Hybridization was started with incubation of the membrane in pre-hybridization solution. It contained reagents which block non-specific DNA binding sites on the membrane surface, reducing background hybridization for $30 \mathrm{~min}$ at $60^{\circ} \mathrm{C}$ or $68^{\circ} \mathrm{C}$. Hybridization was continued after replacement of the pre-hybridization solution by fresh hybridization buffer containing ${ }^{32} \mathrm{P}$-labelled probe. The probe hybridized during an overnight incubation.

The membrane was washed twice with wash solution for $30 \mathrm{~min}$ at $60^{\circ} \mathrm{C}$ or $68^{\circ} \mathrm{C}$. This step gradually removes bound probe molecules until only highly matched hybrids remain. 
The membrane was wrapped in plastic foil for setting up autoradiography, see following next chapter.

After radioautography, the membrane was stripped by boiling for 15 min in $0.1 \%$ SDS; this stripping step was perfomed several times until no radioactivity was left. The membrane was wrapped again and kept at $-20^{\circ} \mathrm{C}$ until the next use.

\subsection{Autoradiography}

In a darkroom, the membrane in plastic wrap was placed on an intensifying screen of calcium tungstate (Dupont). This is a light-tight X-ray film holder to increase the efficiency with which high-energy particles can be detected. The membrane was covered with a sheet of X-ray film from Biomax Kodak.

The film was exposed at $-70{ }^{\circ} \mathrm{C}$ for an appropriate length of time. Thereafter, it was removed for developing in a dark room. The X-ray film was developed in an automatic $X$ ray film processor.

\subsection{Polymerase chain reaction (PCR)}

In vitro enzymatic amplification of a specific DNA segment can be rapidly obtained by the so-called Polymerase Chain Reaction (PCR): A stretch of double-stranded DNA can be amplified provided two single-stranded oligonucleotide primers are flanking it, i.e. bound to the 5-ends of its two thermo-resistant DNA polymerase single strands, is used requiring the 4 deoxyribonucleoside triphosphates (dNTPs) as substrates, a buffer and salts including $\mathrm{MgCl}_{2}$. An enhancer can be used to increase yield, specificity, and to overcome difficulties encountered with regions of a high GC content or with very long templates. These enhancers can be Triton, Tween 20, BSA or DMSO. The PCR reagents have been standardized and appropriate conditions such as temperature and concentrations have been defined for certain primer amplifications.

To obtain a specific good field of the final product, PCR makes use of the exponentially increasing number of templates. Thus, after 30 cycles of replication, a $2^{28}$-fold (270 million-fold) amplification of the discrete product should result.

Each one of these amplification cycles requires a step of 1 . denaturation of DNA molecules at $95^{\circ} \mathrm{C}, 2$. annealing which means hybridization of DNA primers in a temperature range between of $40{ }^{\circ} \mathrm{C}-68{ }^{\circ} \mathrm{C}$, and 3 . extension, the synthesis (replication) of new DNA by DNA polymerase (which catalyzes growth of new strands 
from the $5^{`} \rightarrow 3^{`}$ ends) extending across the segments of original DNA between the new primers at $72{ }^{\circ} \mathrm{C}$.

\subsubsection{Priming conditions and melting temperature}

The primer hybridizations and polymerase reaction should be carried out under temperature conditions favoring the requirements of the enzyme plus a high degree of specificity at a minimum of background; the optimal annealing temperature is at or even above the calculated $T_{m}$ (melting temperature) of the primers making their binding more discriminatory, therefore, more specific. The following formula was used to approximate the $T_{m}$ of primers:

$$
T_{m}=(\mathrm{C}+\mathrm{G}) \times 4^{\circ} \mathrm{C}+(\mathrm{A}+\mathrm{T}) \times 2^{\circ} \mathrm{C}
$$

Table 4.12.1: Components of master mix find concentrations for PCR reaction. The mixture of components for the PCR were prepared according to recipes given under Materials

\begin{tabular}{|l|c|}
\hline Components & Final concentration \\
\hline $10 \times$ PCR buffer & $1 \mathrm{x}$ \\
\hline $10 \mu \mathrm{M}$ Primer $\mathrm{A}$ & $1 \mu \mathrm{M}$ \\
\hline $10 \mu \mathrm{M}$ Primer B & $1 \mu \mathrm{M}$ \\
\hline $25 \mathrm{mM} 4 \mathrm{dNTPs}$ mix & $0.2 \mathrm{mM}$ \\
\hline $25 \mathrm{mM} \mathrm{MgCl}$ & $1.5 \mathrm{mM}$ \\
\hline $100 \times$ Enhancer & $1 \%$ \\
\hline Taq polymerase & $2.5 \mathrm{U} / \mu \mathrm{l}$ \\
\hline Template DNA $^{\mathrm{d}}$ & $100-500 \mathrm{ng} / \mathrm{ml}$ \\
\hline $\mathrm{H}_{2} \mathrm{O}$ up to total volume & - \\
\hline${ }^{\mathrm{d}}$ Enhancer agents (Triton, Tween $\left.20, \mathrm{BSA}, \mathrm{DMSO}\right)$
\end{tabular}

Table 4.12.2 PCR cycling parameters. These cycling parameters were used to perform both PCR and inverse-PCR.

\begin{tabular}{|c|c|c|c|c|}
\hline & Temperature & Step & Time & Cycle \\
\hline 1. & $95^{\circ} \mathrm{C}$ & Denaturation & $2 \min$ & 1 cycle \\
\hline 2. & $94^{\circ} \mathrm{C}$ & Denaturation & $1 \min$ & \multirow{3}{*}{$\begin{array}{c}30 \\
\text { cycles }\end{array}$} \\
\hline 3. & $4^{\circ} \mathrm{C}$ under $T_{m}$ primers & Annealing & $30 \mathrm{sec}$ & \\
\hline 4. & $72^{\circ} \mathrm{C}$ & Extension & $1-3 \min$ & \\
\hline 5. & $72^{\circ} \mathrm{C}$ & Extension & $7 \min$ & 1 cycle \\
\hline 6. & $4^{\circ} \mathrm{C}$ & Cool-stop & unlimited & - \\
\hline
\end{tabular}


To analyze the product, an aliquot of or the total amount of each reaction mixture was loaded onto an agarose gel of the appropriate concentration.

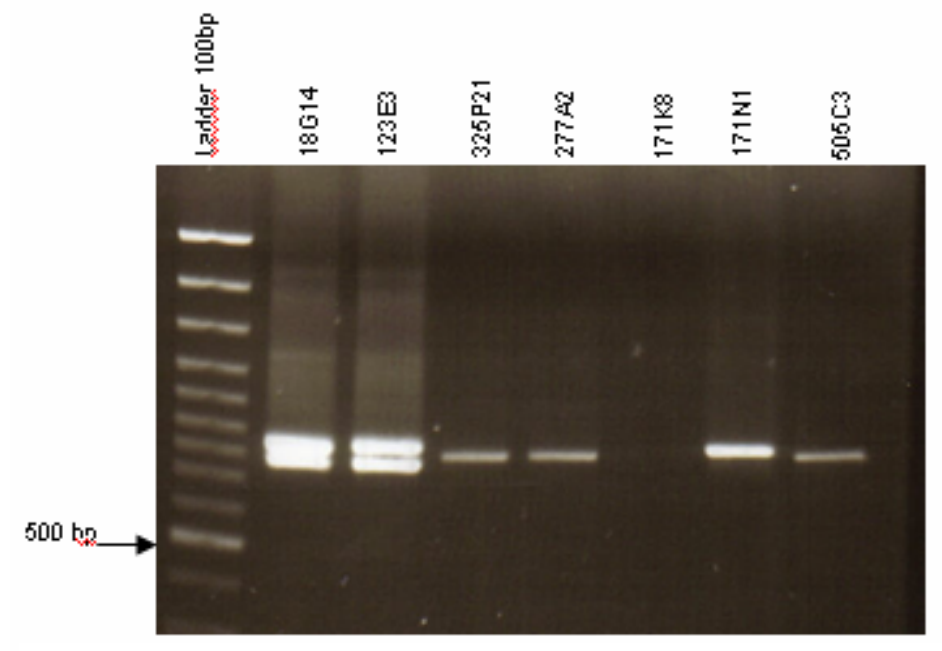

Figure 4.10: Analysis of PCR products by agarose gel electrophoresis.

PCR was carried out with primer HLA-B 29713 to the DNA plasmid of BAC clones samples were loaded into a $1 \%$ agarose gel containing $20 \mathrm{mg} / \mathrm{ml}$ ethidium bromide; ladder marker sizes 100 bp.

\subsection{Inverse PCR}

To amplify DNA, an unknown clone that flanking one end of a known DNA sequence, for which no primers are available inverse PCR or inverted or inside-out PCR is used. The goal of inverse PCR is to generate PCR fragments that contain the end of a BAC insert which can be sequenced. The DNA plasmid was digested by a restriction enzyme, the individual restriction fragments were converted into circles by ligation. The resulting circularized DNA was used as template for inverse PCR. The primers were bound specifically to known sequences in the vector, pointing in opposite directions. Starting at the 3'-end of the bound primer, the subsequent unknown sequence was replicated and amplified in repeated cycles.

For inverse PCR, plasmids were digested with restriction enzyme Pstl and incubated for two hours at $37^{\circ} \mathrm{C}$. The enzyme was inactivated at $65^{\circ} \mathrm{C}$ for $20 \mathrm{~min}$.

Ligation was achieved with T4 Ligase at $16^{\circ} \mathrm{C}$ overnight. The inverse PCR by produced DNA amplification containing the end of the insert just like a normal product of PCR carried out under the same conditions. The primers, used alternatively for inverse PCR were: ptarbac2, ptarbac3, ptarbac4, T7.29 or SP6. 


\subsection{DNA sequencing}

Sequences were found by the Sanger method using the dideoxy-mediated chain reaction coupling with the commercial sequencing kit of the APPLIED BIOSYSTEMS company. A synthetic oligonucleotide primer was annealed to a single stranded DNA template. The reaction mixture contained a small portion of a $2^{\prime}$, 3' $3^{\prime}$-dNTP which carried a $3^{\prime}-\mathrm{H}$ atom on the deoxyribose moiety, rather than the $3^{\prime}-\mathrm{OH}$ group. This molecule marked ddNTP was incorporated into a growing DNA chain, preventing the formation of a phosphodiester bond with the succeeding dNTP. The resulting populations of oligonucleotides were loaded onto a capillary-based automated DNA sequencer (ABI 3700) with the new generation Bigdye. This system incorporates dichlororhodamine dye terminators sequenced with AmpliTaq polymerase resulting in a very complex peak pattern. Therefore, the analysis is automated.

The sequencing reaction occurs in a $20 \mu \mathrm{l}$ final volume including $0.5-1.0 \mu \mathrm{g}$ DNA, $2 \mu \mathrm{l}$ of 10 pmolar primer, $2 \mu$ BigDye, $5 \times$ sequencing buffer and water. The conditions are shown in Table 4.14.1.

Table 4.14.1: Cycling parameters for DNA sequencing. These cycling parameters were used to subsequently perform sequence analysis by $A B I 3700$.

\begin{tabular}{|c|c|l|c|c|}
\hline & Temperature & Step & Time & Cycle \\
\hline 1. & $96^{\circ} \mathrm{C}$ & Denaturation & $2 \mathrm{~min}$ & \multirow{2}{*}{1 cycle } \\
\hline 2. & $96^{\circ} \mathrm{C}$ & Denaturation & $30 \mathrm{sec}$ & \multirow{2}{*}{25 cycles } \\
\cline { 1 - 4 } 3. & $50^{\circ} \mathrm{C}$ & Annealing & $15 \mathrm{sec}$ & \\
\cline { 1 - 3 } 4. & $60^{\circ} \mathrm{C}$ & Extension & $4 \mathrm{~min}$ & \\
\hline
\end{tabular}

The sequencing reaction was ended by precipitation with $250 \mu 100 \%$ ethanol and 10 $\mu \mathrm{l} 3 \mathrm{M}$ sodium acetate $\mathrm{pH}$ 5.5. The mixture was centrifuged at maximal speed for 15 min. Supernatant was discarded with a pipette; the pellet was washed in $200 \mu \mathrm{l} 70 \%$ ethanol and recentrifuged at maximal speed for 5 min. Finally, it was dried in the drypump machine.

Sequencing electropherograms were viewed with EditView 1.0.1 software and Bioedit program. The sequence analyses were realized with Clustal $X$ version 1.8 for alignments and sequence identity (see Figure 4.11). 


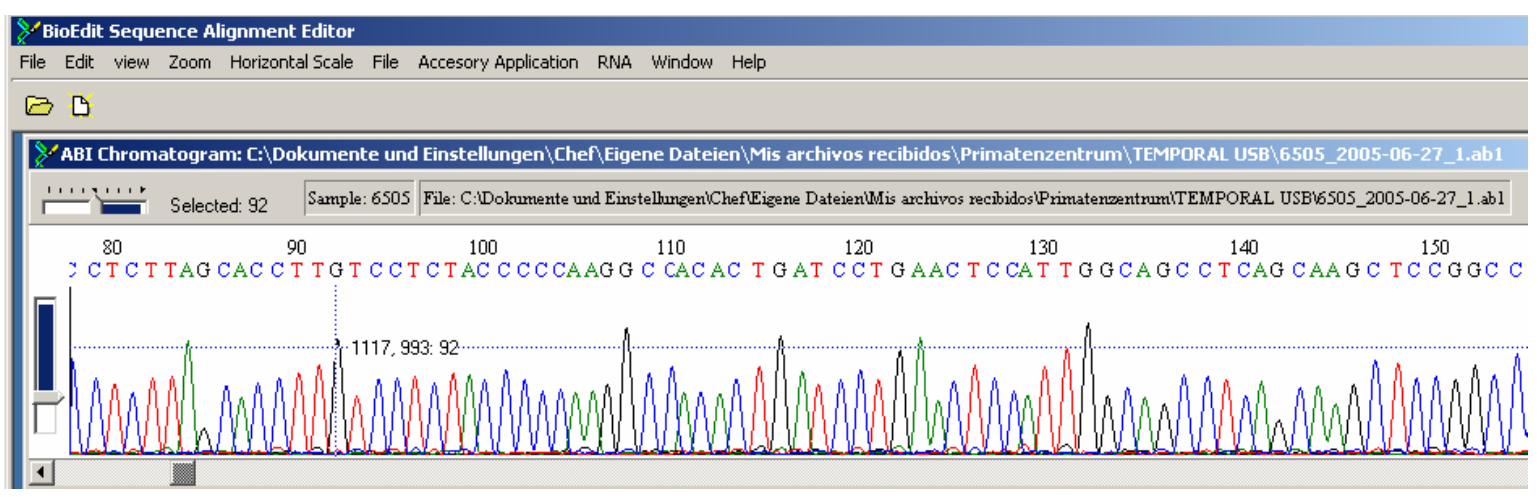

Figure 4.11: Sequencing electropherogram of a DNA fragment from a BAC clone. The sequence was performed in the $A B I$ 3100. Sequence analysis was viewed with bioedit equipped with editView 1.0.1 software from Applied Biosystems.

The sequences were translated in BLAST and were analyzed in Swiss-prot. To resolved some difficult in the sequence analysis was used AUGUSTUS software. The evolution analysis with sequences was realized with Clustal X, Genrunner version 3.05 and MEGA 3.1. 


\section{Results}

The results of these pilot studies on the MHC class I region in Callithrix jacchus, on gene order and DNA sequences, their location, assignment and eventually interpretation are reported essentially in the sequence of their production. A table of contents indicating page numbers is placed behind the title on this thesis.

This technical note may help to read the Results chapters: A solution of the layout problem of combining a fairly brief text with documents showing the results, i.e. numerous figures and tables each filling often a whole page or more, shall be attempted by giving in the beginning of chapter a short introductory text providing a survey of the subjects to be delft with. Detailed yet brief explanations will then be given in the legends for enabling study of the data presented right next to these. A Conclusions paragraph may be added, where adequate, at the end of the chapter. Only discussion proper, of problems, criticisms, observations seeming of general interest, and ideas hopefully leading further, is left for the discussion section.

Although it is needed as seen from various aspects mentioned, no information is available on the number, order and clustering of genes in the MHC class I region of the Common marmoset. Therefore, the first aim was to construct a physical map showing a sequence of genes in the MHC class I region based upon fragment analysis of clones from the BAC bank CHORI-259.

The work finally resulted in a partial description of the order of MHC class I genes, especially in contig 1(Figure 5.2.1.3.1). In consideration of the much greater value of sequence data - compared to the indirect evidence of such fragment-gene assignments, - and in view of the facilities existing to do sequence analysis and had decided in time to start a partial sequencing of the MHC class I region. BAC clones selected (5.1) for making the first aim (5.2) could thus be tested also for usefulness in sequencing by PCR, i.e. following the second aim (5.3). The partial sequences obtained were localized by comparative alignment in a Hs MHC class I region map drawn to bp-scale (5.4); both complemented the results of project 1 and allowed a study on evolution of sequences (5.5). Clustal $X$ and MEGA 3.1 programs for constructing phylogenetic trees of genes could be used to reversely identify unknown class I pseudogenes within unassigned DNA sequences, indicating the nearest relatives of these genes (5.6). Knowledge of the sequences alignment and gene contents may serve, furthermore, as milestones on the way of a complete sequencing of the MHC class I region of Callithrix jacchus. 
Sequence TS1 turned out to be, however, the most instructive and challenging one. It might be once considered a "Rosette stone" for immunogenetics, a key to the evolution of the classical class I gene duplicons.

\subsection{Identification of BAC clones from Callithrix jacchus containing MHC class I region gene}

The BAC clones containing class I information were identified e.g with Caja-G a probe of cDNA from Callithrix jacchus prepared in $\mathrm{P}^{32}$ labelled form: MHC class I Caja-G a fragment of 734bp of clone 15 of liver A389 cDNA. It contains exon 2 with 272bp (polymorphic), exon 3 with 275bp (polymorphic) and exon 4 with 187bp (conserved). This probe is named Caja-G had been kindly provided by Ulrike Geisler. The MHC class I gene complex has shown homology between human, rat, mouse, pig, cat, monkeys like Rhesus, horse and other mammals (Amadou et al., 1999; Trowsdale, 1999; Daza-Vamenta et al., 2004; Hurt et al, 2004; Beck et al., 2005; Chardon et al., 2000; Tallmadge et al., 2005). The MHC class I probe Caja-G was chosen in recognition of the fact that MHC class I proteins have homology not only between species but also with other members of the HLA family of genes, and to a lesser extent with their pseudogenes found within gene clusters of class I region. Caja-G has a high degree of identity with the other class I probe used, called HLA-B, i.e. taken from Macaca mulatta (Rhesus monkey), both monitoring the same fragments in hybridization. MICA, a third class I probe, does not cross-react with both Caja-G and HLA-B in hybridization.

For a physical mapping, all clones of BAC Bank CHORI-259 were screened. This bank contains the complete genome of Callithrix jacchus incorporated at an 11.2-fold genomic representation into PTARBAC 2.1 vector clones of E.coli (Materials, 3.9.).

An initial screening of the library with the Caja-G probe yielded 256 positive BAC clones. Framework gene sequences within the isolated BAC clones were demonstrated also by hybridization. The probes used contain so-called framework genes, conserved sequences belonging to the $\mathrm{MHC}$ organization in humans and in rats from which they had been obtained (Amadou et al., 1995). Altogether, they are flanking all four intervals containing class I and other genes. In Hs these intervals are arranged in the following manner: BAT1 - POU5F1, TCF19 - CAT56, GNL1 (TRIM39, TRIM26) - TCTEX5 and TCTEX4 - MOG (MHC class I map, text and Figure 5.1.1). A probe POU5F1 was included, because the TCF19 framework probe 
did not hybridize with small restriction fragments nor in the final Southern blots - in contrast to the whole clones which did so. A probe of K18T3 was generated, furthermore, in an attempt of closing a gap within contig 1. Of course, it has a unique specificity in hybridization, not cross-reacting with the class I probes.

153 BAC clones were found by hybridization to contain MHC class I gene(s) and MIC gene(s), respectively. For establishing a genomic map for the MHC class I region of Callithrix jacchus, 42 clones were allocated to the four class I intervall, most of these (31) belonging to contig 1 . See of their probe-hybridizations are summarized in table 5.2.1.2. 


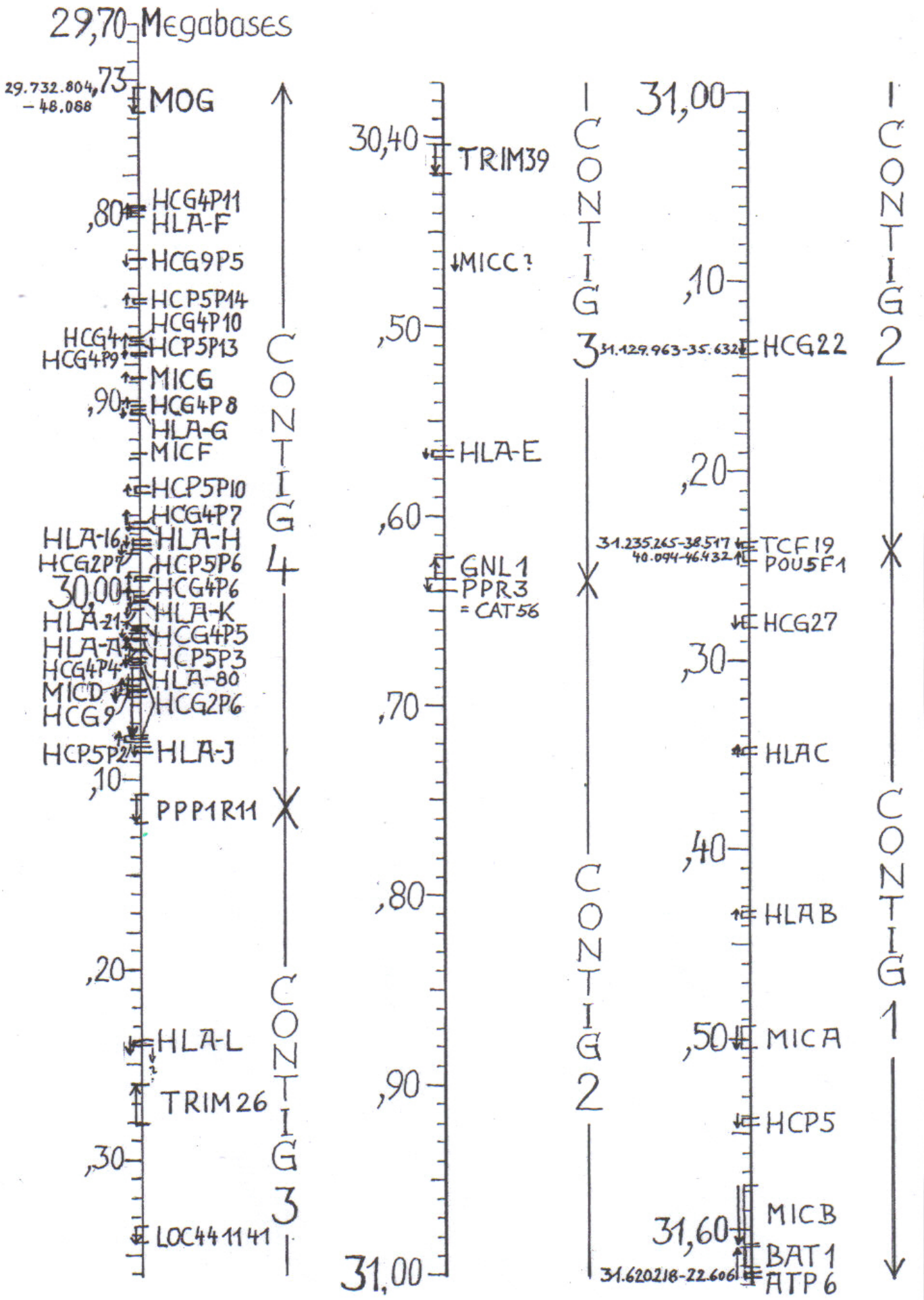

Figure 5.1: Map of the MHC class I region in Hs showing to bp-scale the arrangement of four intervals called contigs 1 - 4 .

The class I protein genes are clustered (scarcely in contigs 2 and 3 ) between conserved framework genes (marked green in figure 5.4.1). Figure made by Prof. Johannes Heinrich Matthaei. 


\subsubsection{Contig 1, the interval BAT1 - TCF19}

The interval between BAT1 and TCF19 is polymorphic. Polymorphic applies to regions with genetic alterations between species, e.g. in respect to the MHC class I region with the genes $H L A-B, H L A-C$ and $M I C A / B$ in several mammalian species. Double positive screening of BAC clones was registered between $\mathrm{MHC}$ class I gene Caja-G* and the following probes: ATP6V1g2, BAT1, TCF19, HLA-B, POU5F1, MICA and K18T3 (see table 5.1.1.1).

${ }^{*}$ ) Following a lab routine, in this text, the probe is named occasionally MHC class I instead of MHC-class I-Caja-G or just Caja-G that is precise and sufficient.

\section{Table 5.1.1.1: Screening of the interval between BAT1 and TCF19}

The 11 filters of BAC-bank CHORI-259 were screened with the BAT1, TCF19, POU5F1, MICA, K18T3, HLA-B and MHC class I probes, respectively. Numbers of the clones matching with the indicated groups of these probes, tested individually, are listed.

\begin{tabular}{|l|c|}
\hline \multicolumn{1}{|c|}{ Clones positive to probes } & No. of clones \\
\hline TCF19, MHC class I & 11 \\
\hline BAT1, MHC class I & 24 \\
\hline TCF19, BAT1 & 45 \\
\hline TCF19, BAT1, MHC class I & 18 \\
\hline POU5F1, MHC class I, TCF19 & 5 \\
\hline POU5F1, BAT1, MHC class I & 4 \\
\hline POU5F1, BAT1 & 2 \\
\hline POU5F1, HLA-B, MHC class I & 2 \\
\hline POU5F1, TCF19, MHC class I & 5 \\
\hline POU5F1, BAT1, MHC class I & 4 \\
\hline K18T3, MHC class I & 1 \\
\hline K18T3, BAT1, TCF19, MHC class I & 1 \\
\hline K18T3, BAT1, MICA, HLA-B, MHC class I & 1 \\
\hline K18T3, HLA-B, TCF19 & 1 \\
\hline K18T3, BAT1 & 1 \\
\hline K18T3, MICA, HLA-B, MHC class I & 1 \\
\hline K18T3, MICA & 1 \\
\hline K18T3, HLA-B & 6 \\
\hline K18T3, HLA-B, MHC class I & 21 \\
\hline K18T3, HLA-B, TCF19, MHC class I & 1 \\
\hline K18T3, HLA-B, BAT1, MHC class I & 4 \\
\hline
\end{tabular}

From the BAC clones screening with BAT1, TCF19 and MHC class I probes, respectively, 31 were selected for characterization of their fragments by Southern blot fingerprinting and sequence analysis. These are listed in table 5.1.1.2. 
Table 5.1.1.2: Screening into the interval between BAT1 and TCF19 by means of BAC-bank CHORI-259.BAC-clones are characterized by their filter number, plate and coordinates. All probes from other mammals tested here, showed complementarily with BAC clone DNA from Callithrix jacchus.

\begin{tabular}{|c|c|c|c|c|c|}
\hline Filter & $\begin{array}{l}\text { Plate and } \\
\text { coordinates }\end{array}$ & Positive probe & $\begin{array}{l}\text { Positive } \\
\text { probe }\end{array}$ & $\begin{array}{l}\text { Positive } \\
\text { probe }\end{array}$ & $\begin{array}{c}\text { Positive } \\
\text { probe }\end{array}$ \\
\hline 031 & 120D3 & MHC class I & TCF19 & BAT1 & \\
\hline 061 & $271 \mathrm{C5}$ & MHC class I & TCF19 & BAT1 & \\
\hline 031 & 127E13 & MHC class I & TCF19 & BAT1 & \\
\hline 061 & 261L9 & MHC class I & TCF19 & BAT1 & \\
\hline 011 & $18 G 14$ & & TCF19 & BAT1 & \\
\hline 07G & 282L12 & & TCF19 & BAT1 & \\
\hline $05 I$ & $204 C 3$ & & TCF19 & BAT1 & \\
\hline 051 & 239N18 & & TCF19 & BAT1 & \\
\hline 051 & 193P12 & & TCF19 & BAT1 & \\
\hline 061 & 282015 & & TCF19 & BAT1 & \\
\hline 011 & 44G8 & MHC class I & & BAT1 & \\
\hline $011 \mathrm{G}$ & $510 K 19$ & & TCF19 & BAT1 & MICA \\
\hline $05 I$ & $217 \mathrm{M} 17$ & MHC class I & & & MICA \\
\hline $04 \mathrm{I}$ & $161 \mathrm{C} 12$ & & & BAT1 & MICA \\
\hline 041 & 174N1 & MHC class I & & & MICA \\
\hline $08 \mathrm{G}$ & $347 \mathrm{~K} 7$ & MHC class I & TCF19 & HLA-B & POU5F1 \\
\hline 08G & 347D1 & MHC class I & TCF19 & HLA-B & POU5F1 \\
\hline 08G & 379F14 & MHC class I & TCF19 & & POU5F1 \\
\hline 09G & $425 \mathrm{~A} 13$ & MHC class I & TCF19 & & POU5F1 \\
\hline 10G & 459020 & MHC class I & TCF19 & & POU5F1 \\
\hline 061 & $261 \mathrm{~K} 9$ & MHC class I & & BAT1 & POU5F1 \\
\hline 041 & $169 \mathrm{G} 2$ & MHC class I & & BAT1 & POU5F1 \\
\hline 031 & $123 \mathrm{H} 17$ & MHC class I & & BAT1 & POU5F1 \\
\hline 031 & 123E17 & MHC class I & & BAT1 & POU5F1 \\
\hline 041 & 150M18 & & & BAT1 & POU5F1 \\
\hline $03 \mathrm{I}$ & 99L19 & & & BAT1 & POU5F1 \\
\hline 07G & $334 C 4$ & MHC class I & K18T3 & HLA-B & \\
\hline $07 \mathrm{G}$ & 329M5 & MHC class I & K18T3 & HLA-B & \\
\hline 08G & $379 \mathrm{E} 3$ & MHC class I & K18T3 & HLA-B & \\
\hline 041 & 174N1 & MHC class I & K18T3 & HLA-B & MICA \\
\hline $011 \mathrm{G}$ & $485 \mathrm{C} 4$ & & K18T3 & & MICA \\
\hline
\end{tabular}

\subsubsection{Contig 3, the intervals TRIM39 - TRIM26}

The interval TRIM39 - TRIM26 of the MHC class I region is polymorphic in human. Their existence in Callithrix jacchus was demonstrated by double positive screening of the MHC class I probe Caja-G, with probes CAT56, TRIM39 and TRIM26 probes, respectively (see Figure 5.2.2.3). In this screening of the interval CAT56-TRIM39, 20 positive clones were found (Table 5.1.2.1). For the MHC class I-TRIM39-TRIM26 interval, 21 positives clones were detected (Table 5.1.2.2). 
Table 5.1.2.1: Screening for clones containing Caja-G and frameworks genes of CAT56 - TRIM39 (part of contig 3).

\begin{tabular}{|l|c|l|}
\hline $\begin{array}{c}\text { Group of probes individually } \\
\text { matching BAC clones }\end{array}$ & $\begin{array}{c}\text { No. of BAC clones } \\
\text { positive with each } \\
\text { probe of the group }\end{array}$ & \multicolumn{1}{|c|}{ Clones found on filters $^{*}$} \\
\hline CAT56, Caja-G & 9 & 03I, 04I, 05I, 06(3), 07G (2), 09G \\
\hline TRIM39, CAT 56 & 3 & 06I(2), 07G \\
\hline TRIM39, Caja-G & 9 & $01 \mathrm{I}, 05 \mathrm{I}(3), 06 \mathrm{I}, 07(3), 09 \mathrm{G}, 010 \mathrm{G}(3)$ \\
\hline CAT56, TRIM39, Caja-G & 1 & $07 \mathrm{G}$ \\
\hline TRIM39, TRIM26, CAT 56 & 3 & 06I(2), 07G \\
\hline
\end{tabular}

* In parentheses: number of clones on filter mentioned

One BAC clone, number 325P21 on filter 7 (07G), was found to be screened with four framework probes, MHC class I Caja G, CAT56, TRIM39 and TRIM26.

BAC clone 325P21 was selected for characterization by Southern blot fingersprints. All other framework probes (7) flanking the MHC class I region were positive.

8 BAC clones were chosen for screening with TRIM39, TRIM26 and MHC class I in order to characterize their matching DNA by Southern blot fingersprints, see table 5.1.2.3.

For control, Southern blot analysis was done also with nylon membranes carrying clones screening for the BAT1-TCF19 interval. All these clones were negative (Table 5.1.2.3).

Of the BAC clones positive in screening to TRIM39, TRIM26 and MHC class I chosen eight were chosen for characterization by Southern blot fingerprints; clone 325P21 was not included.

In Southern blot analysis, membranes with clones containing interval BAT1-TCF19 were tested for hybridization with probes TRIM39, TRIM26, POU5F1, HLA-B, CAT56, TCTEX5, TCTEX4 and MOG. All of these controls were negative (Table 5.1.2.3).

Table 5.1.2.2 Screening for clones containing Caja-G and the interval TRIM39 TRIM26 (part of contig 3).

\begin{tabular}{|l|c|l|}
\hline $\begin{array}{c}\text { Group of probes individually } \\
\text { matching BAC clones }\end{array}$ & $\begin{array}{c}\text { No. of BAC clones } \\
\text { positive with each } \\
\text { probe of the group }\end{array}$ & \multicolumn{1}{|c|}{ Clones found on filters * } \\
\hline TRIM39, TRIM26 & 14 & $01 \mathrm{I}, 05 \mathrm{I}(2), 06 \mathrm{I}(5), 07 \mathrm{G}(2), 010 \mathrm{G}(2), 011 \mathrm{G}(2)$ \\
\hline TRIM26, Caja-G & 11 & $03 \mathrm{I}, 04 \mathrm{I}(2), 05 \mathrm{I}(2), 06 \mathrm{I}, 07 \mathrm{G}, 010 \mathrm{G}(4)$ \\
\hline TRIM39, Caja-G & 9 & $01 \mathrm{I}, 05 \mathrm{I}(2), 06 \mathrm{I}, 07 \mathrm{G}(2), 09 \mathrm{G}, 010 \mathrm{G}(2)$ \\
\hline TRIM26, TRIM39, Caja-G & 7 & $01 \mathrm{I}, 05(2), 06 \mathrm{I}, 07 \mathrm{G}, 010 \mathrm{G}(2)$ \\
\hline
\end{tabular}


Table 5.1.2.3: Screening into the intervals CAT56 - TRIM39 and TRIM39 TRIM26 by means of BAC-bank-bank CHORI-259: Each BAC-clone is characterized by its filter number and plate coordinates. All the probes from other mammals tested showed complementarily, with BAC clone DNA from Callithrix jacchus.

\begin{tabular}{|c|c|c|c|c|c|}
\hline BAC clones & Filter No. & $\begin{array}{l}\text { Positive with } \\
\text { probe }\end{array}$ & $\begin{array}{l}\text { Positive with } \\
\text { probe }\end{array}$ & $\begin{array}{c}\text { Positive } \\
\text { with probe }\end{array}$ & $\begin{array}{c}\text { Positive } \\
\text { with probe }\end{array}$ \\
\hline $245 \mathrm{C} 6$ & 061 & Caja-G & TRIM26 & TRIM39 & --- \\
\hline 234L16 & 051 & Caja-G & TRIM26 & TRIM39 & --- \\
\hline $463 N 5$ & $010 \mathrm{G}$ & Caja-G & TRIM26 & TRIM39 & --- \\
\hline $19 \mid 16$ & 011 & Caja-G & TRIM26 & TRIM39 & --- \\
\hline 325P21 & $07 \mathrm{G}$ & Caja-G & TRIM26 & TRIM39 & CAT56 \\
\hline $279 K 21$ & 061 & --- & TRIM26 & TRIM39 & --- \\
\hline $277 \mathrm{~A} 2$ & 061 & --- & TRIM26 & --- & ---- \\
\hline 436B4 & 010G & Caja-G & TRIM26 & --- & --- \\
\hline
\end{tabular}

\subsubsection{Contig 4, interval TCTEX4 - MOG}

The interval was investigated using 15 BAC clones for multiple positive screenings with probes Caja-G, HLA-B, TCTEX4 and MOG (Table 5.1.3.1). The contig was constructed by means of fragments from two of these15 clones (see Figure $5 \cdot 2 \cdot 3 \cdot 3 \cdot 1)$.

Table 5.1.3.1: Screening of the interval TCTEX4 - MOG:

\begin{tabular}{|c|c|c|}
\hline $\begin{array}{c}\text { Group of probes individually } \\
\text { matching BAC clones }\end{array}$ & $\begin{array}{c}\text { No. of BAC } \\
\text { clones positive } \\
\text { with each probe } \\
\text { of the group }\end{array}$ & $\begin{array}{c}\text { Clones found on filters (No. of } \\
\text { clones) }\end{array}$ \\
\hline TCTEX4, Caja-G & 8 & 01I, 03I, 04I, 07G,010G,011G(3) \\
\hline MOG, Caja-G & 3 & $01 \mathrm{I}, 04 \mathrm{I}, 08 \mathrm{G}$ \\
\hline MOG, TCTEX4 & 5 & $06 \mathrm{I}, 08 \mathrm{G}, 011 \mathrm{G}(3)$ \\
\hline Caja-G, MOG, TCTEX4 & 1 & $01 \mathrm{I}$ \\
\hline
\end{tabular}


Table 5.1.3.2: Screening into the interval TCTEX4 - MOG by means of BACbank CHORI-259 clones with probes TCTEX4, MOG, Caja-G, HLA-B and MICA: Each BAC-clone is characterized by its plate coordinates and filter number. All the probes from other mammals showed hybridization with BAC clone DNA from Callithrix jacchus.

\begin{tabular}{|c|c|c|c|c|c|}
\hline BAC clones & Filter No. & $\begin{array}{c}\text { Positive } \\
\text { with probe }\end{array}$ & $\begin{array}{c}\text { Positive } \\
\text { with probe }\end{array}$ & $\begin{array}{c}\text { Positive } \\
\text { with probe }\end{array}$ & $\begin{array}{c}\text { Positive } \\
\text { with probe }\end{array}$ \\
\hline 348C21 & $08 \mathrm{G}$ & Caja-G & TCTEX4 & MOG & HLA-B \\
\hline 171K8 & 041 & Caja-G & TCTEX4 & MOG & HLA-B \\
\hline
\end{tabular}

\subsection{Genomic analysis by Southern blot hybridization}

To determine with BAC clones the arrangement of genes within contigs of the MHC class I region, EcoRI digestion fingerprints of DNA from the 42 selected BAC clones were probe-hybridized in Southern blots for the analysis of gene content of the fragments. With the relatively few clones used, only three contigs were found in the MHC class I regional organization using 10 different framework specific probes, the MHC class I probes Caja-G, HLA-B from Mamu and MICA, furthermore, a probe for the gene K18T3. All hybridization results are collected in table 5.2.1.2.2 summarizing the data from all 42 clones used for representation of contigs 1, 3 and 4 . It contains the clones of fragments split from the clone's inserts by the EcoRI restriction enzyme, and their probe-screening.

\subsubsection{Contig 1, the interval BAT1 - TCF19}

\subsubsection{Southern blot analysis of contig 1}

The genes in this contig turned out to be the same as in human, mouse, rat and chimpanzee. As intended initially, this contig should have included the interval between the framework genes BAT1 and TCF19. TCF19 is localized next to POU5F1. 10 clones by screening results covering BAT1 - TCF19 were identified by hybridization on figerprintes. These BAC clones are: 510K19, 261L9, 204C3, 161C12, 44G8, 171N, 217M17, 485C4, 334C3 and 329M5. With these, 13 probe Caja-G hybridizing fragments were found for contig 1 between BAT1 and POU5F1. But it seemed difficult to assemble all of these into a map. Only the four fragments of 4.2, 9, 10 and $11 \mathrm{~kb}$ could be positioned. The HLA-B probe (Mamu-B) was found positive with $7 \mathrm{EcoR}$ fragments. Of these, the fragments of 6 and $7 \mathrm{~kb}$ did not seem to fit in. At least 8 class I genes were expected to be located in the region between 
BAT1 and POU5F1. The MICA probe hybridized to an ECORI fragment with length 17 $\mathrm{kb}$ in the clones 510K19, 204C3, 261C12, 161C12, 44G8, 217M17, 171N1, 485C14, $334 \mathrm{C} 4$ and 329M5.

Initial screening of contig 1 presented the problem of TCF19 hybridizing in clone screening, however, not in EcoRl fragments of the clones. TCF19 is located between POU5F1 and HCR. A Southern blot analysis for framework gene HCR was negative in all clones, too. To close this gap between contig 1 and contig 2, screening with a POU5F1 probe was done. 10 clones were found POU5F1-positive: 379F14, 425A14, 459O20, 261K9, 169G2, 123H17, 150M18, 99L19, 317K7 and 347D1. Eigth of these clones were bought and found POU5F1-positive also in Southern blot analysis: 379F14, 425A14, 459O20, 169G2, 123H17, 99L19, 317K7 and 347D1. However, there was no clone covering HLA-B and POU5F1. In the PUO5F1-screening clones, two fragments of 10 and $11 \mathrm{~kb}$ were found both of which were positive for Caja-G and HLA-B probes. The assumption was that HLA-B/Caja-G-related and therefore hybridizing genes would be located only in or near the gene HLA-B. In the aim to close a gap between HLA-B and PUO5F1, a primer called K18T3 was prepared from clone $217 \mathrm{M} 17$. With this primer, a probe was made and used for screening the 11 filters of the BAC bank. In this screening, 5 clones were found: 174N1, 217M17, $485 \mathrm{C} 4,329 \mathrm{M} 5$ and $334 \mathrm{C} 4$. Southern blot analysis of these clones showed a $17 \mathrm{~kb}$ fragment positive for MICA. Therefore, the following clones overlap both K18T3 and MICA: $174 \mathrm{~N} 1,485 \mathrm{C} 4,329 \mathrm{M} 5$ and 334C4. Overlapping to HLA-B was indicated by a fragment of $9 \mathrm{~kb}$ being positive for both Caja-G and Mamu-B probes. Clone 329M5 had two fragments, of 9 and $2.3 \mathrm{~kb}$, both positive for Caja-G and Mamu-B probes. The clones $334 \mathrm{C} 14$ and $379 \mathrm{E} 3$ overlapped with fragments of 4.2 or $2.2 \mathrm{~kb}$, respectively, with K18T3; with fragments of $11 \mathrm{~kb}$ (in addition, of $4.2 \mathrm{~kb}$ in $379 \mathrm{E} 3$ and 18G149) positive for Caja-G and Mamu-B probes. That means, these clones are in the cluster containing fragments positive for Caja-G/Mamu-B and POU5F1. The clones positive for probe K18T3 screened to close the gap between HLA-B and the framework gene POU5F1.

All fragment - gene-probe - assignments obtained for the clones used for restriction mapping are collected in table 5.2.1.2.1 providing the basis for construction of maps, mainly for the interval BAT1 - POU5F1, contig 1. 
A final construction of the contig 1 map led to the conclusion that class I (=Caja-G) positive fragments must be assumed to occur on both sides of K18T3. This probe finally, turned out to be useful, but not absolutely necessary for obtaining the map.

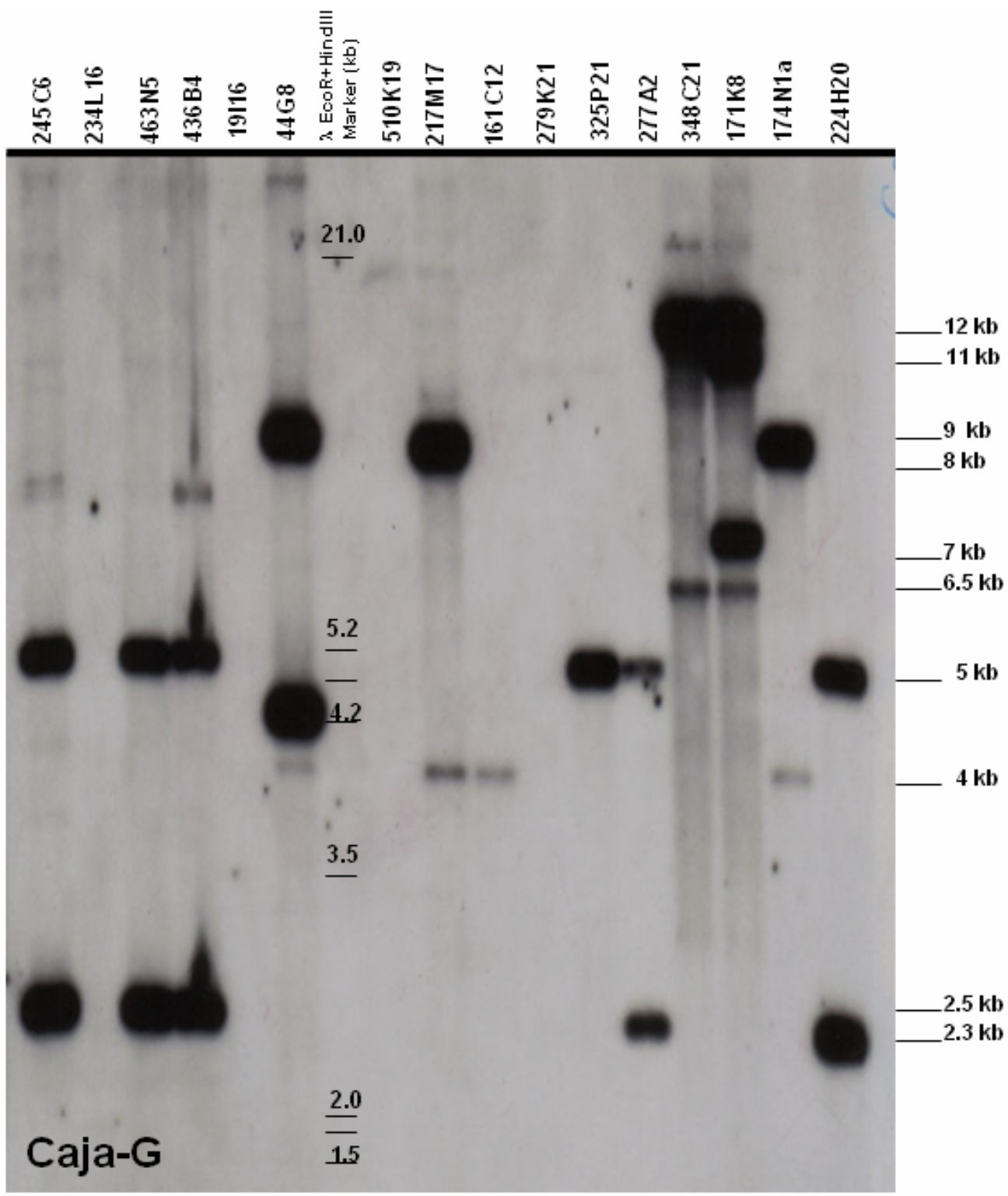

Figure 5.2.1.1.1: Autoradiograph after Southern blot hybridization of Caja-G probe with BAC-clone fragments (CHORI-259, Callithrix jacchus).

MHC-class I-positive BAC-clones were digested with restriction enzyme EcoRI. After gel electrophoresis and fingerprinting, their fragments were hybridized with said gene probe. DNA sample Lambda $(\lambda)$ EcoR/+HindIII used as ladder marker. kb values on the right margin calculated relative to marker DNA fragments which were seen by means of ethidium bromide fluorescence. 


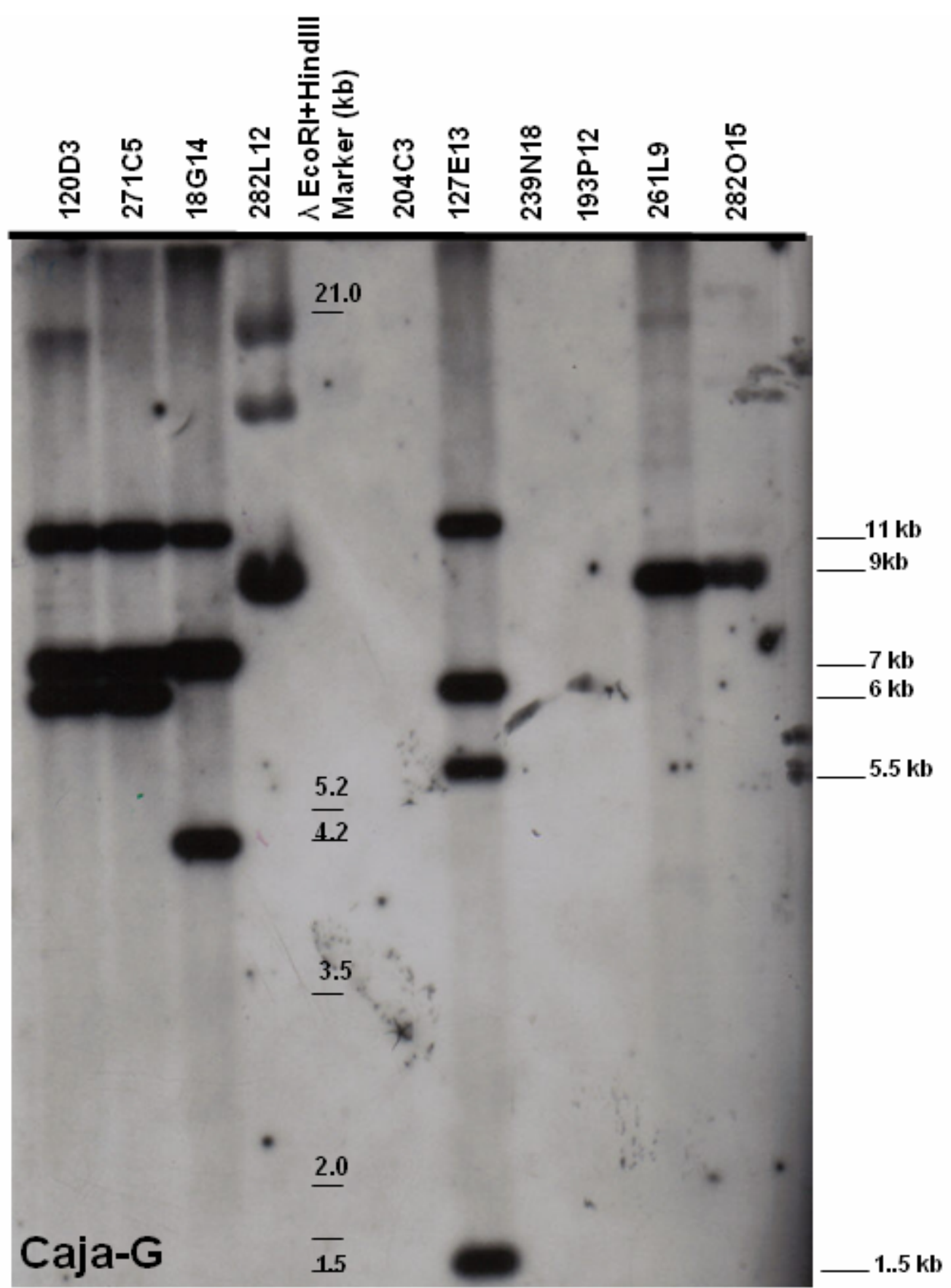

Figure 5.2.1.1.2: Autoradiograph after Southern blot hybridization of Caja-G probe with BAC-clone fragments (CHORI-259, Callithrix jacchus).

MHC-class I-positive BAC-clones were digested with restriction enzyme EcoRI. After gel electrophoresis and fingerprinting, their fragments were hybridized with said gene probe. DNA sample Lambda $(\lambda)$ EcoRI+HindIII used as ladder marker. kb values on the right margin calculated relative to marker DNA fragments which were seen by means of ethidium bromide fluorescence 


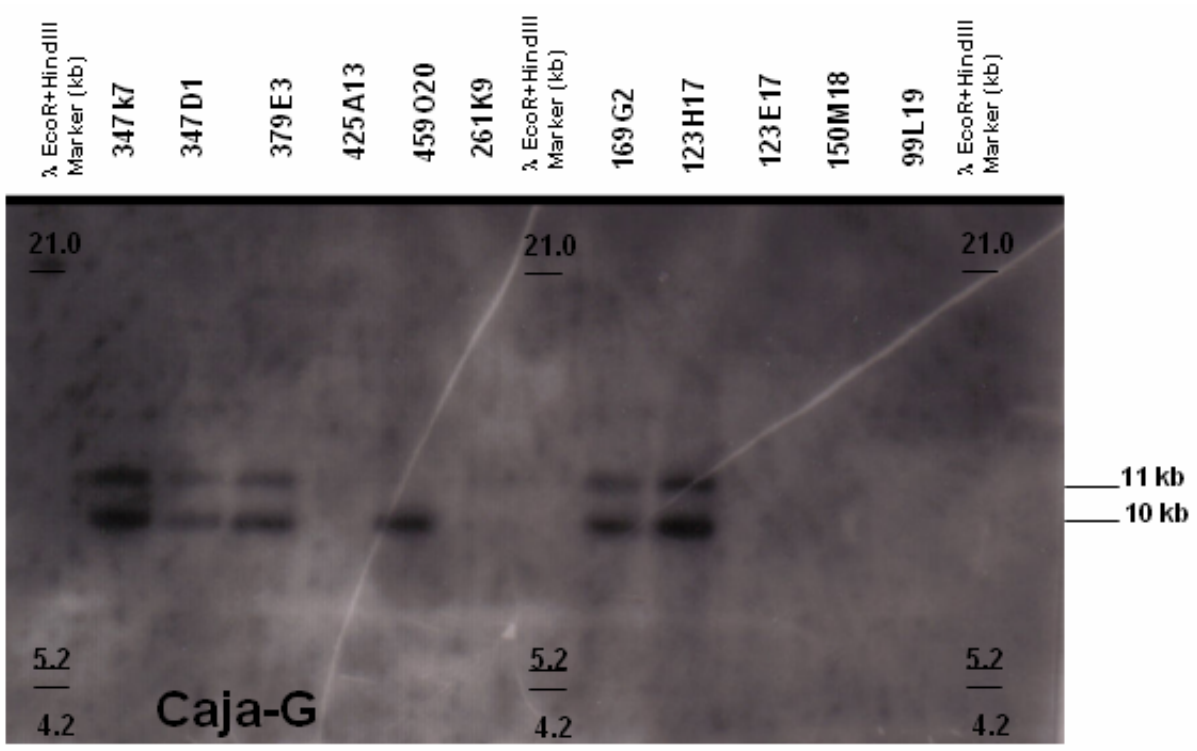

Figure 5.2.1.1.3 Autoradiograph after Southern blot hybridization of Caja-G probe with BAC-clone fragments (CHORI-259, Callithrix jacchus).

MHC-class I-positive BAC-clones were digested with restriction enzyme EcoRI. After gel electrophoresis and fingerprinting, their fragments were hybridized with said gene probe. DNA sample Lambda $(\lambda)$ EcoRI+HindIII used as ladder marker. kb values on the right margin calculated relative to marker DNA fragments which were seen by means of ethidium bromide fluorescence.
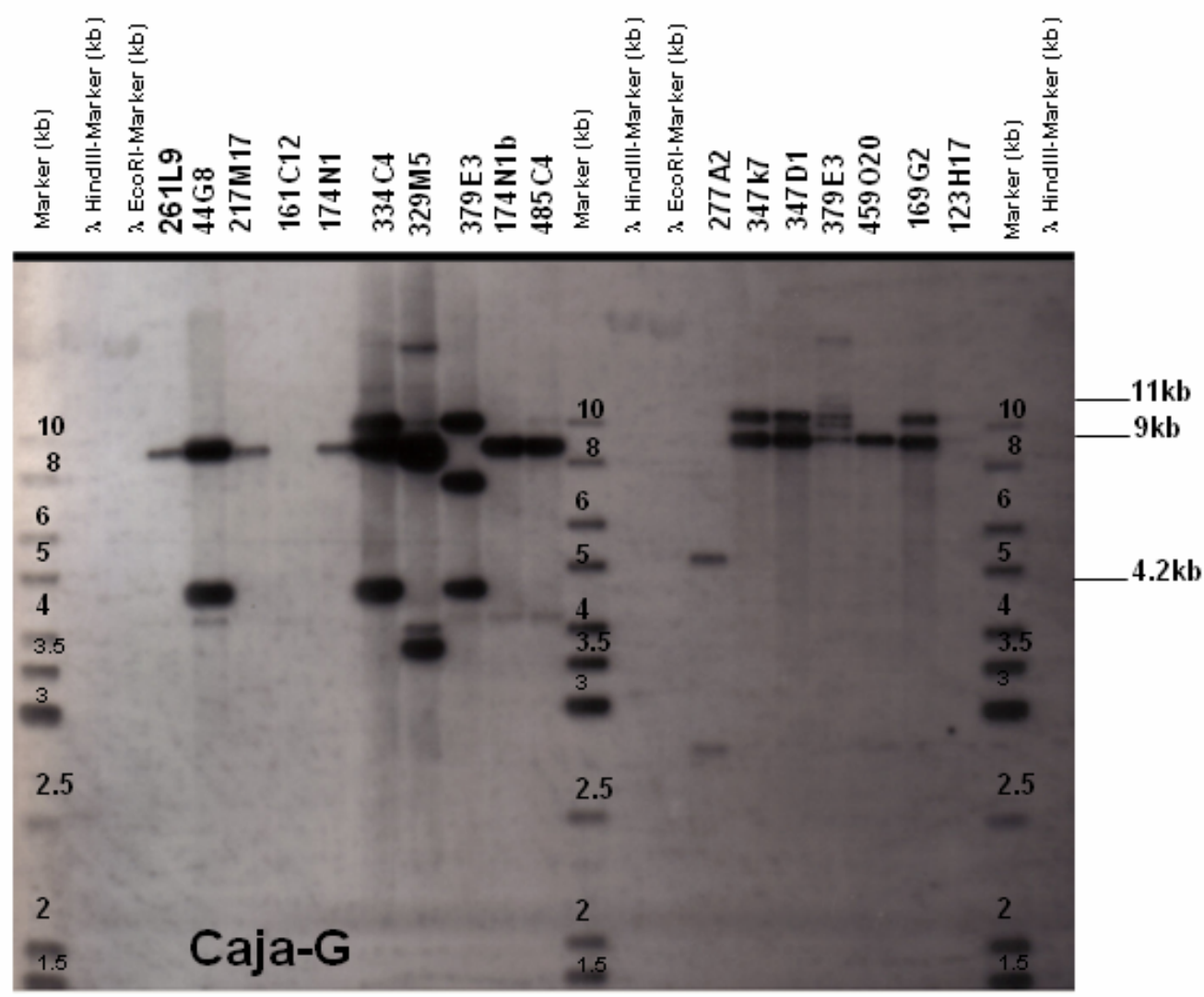
Figure 5.2.1.1.4: Autoradiograph after Southern blot hybridization of Caja-G probe with BAC-clone fragments (CHORI-259, Callithrix jacchus).

MHC-class I-positive BAC-clones were digested with restriction enzyme EcoRI. After gel electrophoresis and fingerprinting, their fragments were hybridized with said gene probe. DNA sample Lambda $(\lambda)$ EcoRI+HindIII used as ladder marker. kb values on the right margin calculated relative to marker DNA fragments which were seen by means of ethidium bromide fluorescence.

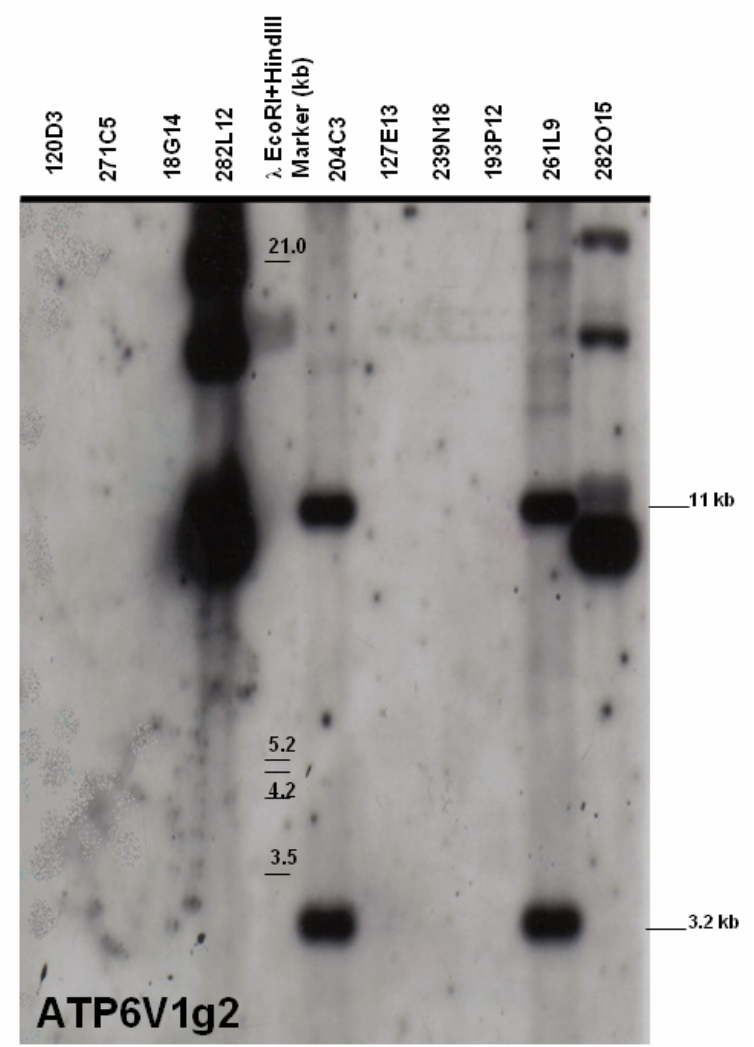

Figure 5.2.1.1.5: Autoradiograph after Southern blot hybridization of ATP6V1g2 framework gene probe with BAC-clone fragments (CHORI-259, Callithrix jacchus).

MHC-class I-positive BAC-clones were digested with restriction enzyme EcoRI. After gel electrophoresis and fingerprinting, their fragments were hybridized with said gene probe. DNA sample Lambda $(\lambda)$ EcoRI+HindIII used as ladder marker. kb values on the right margin calculated relative to marker DNA fragments which were seen by means of ethidium bromide fluorescence. 


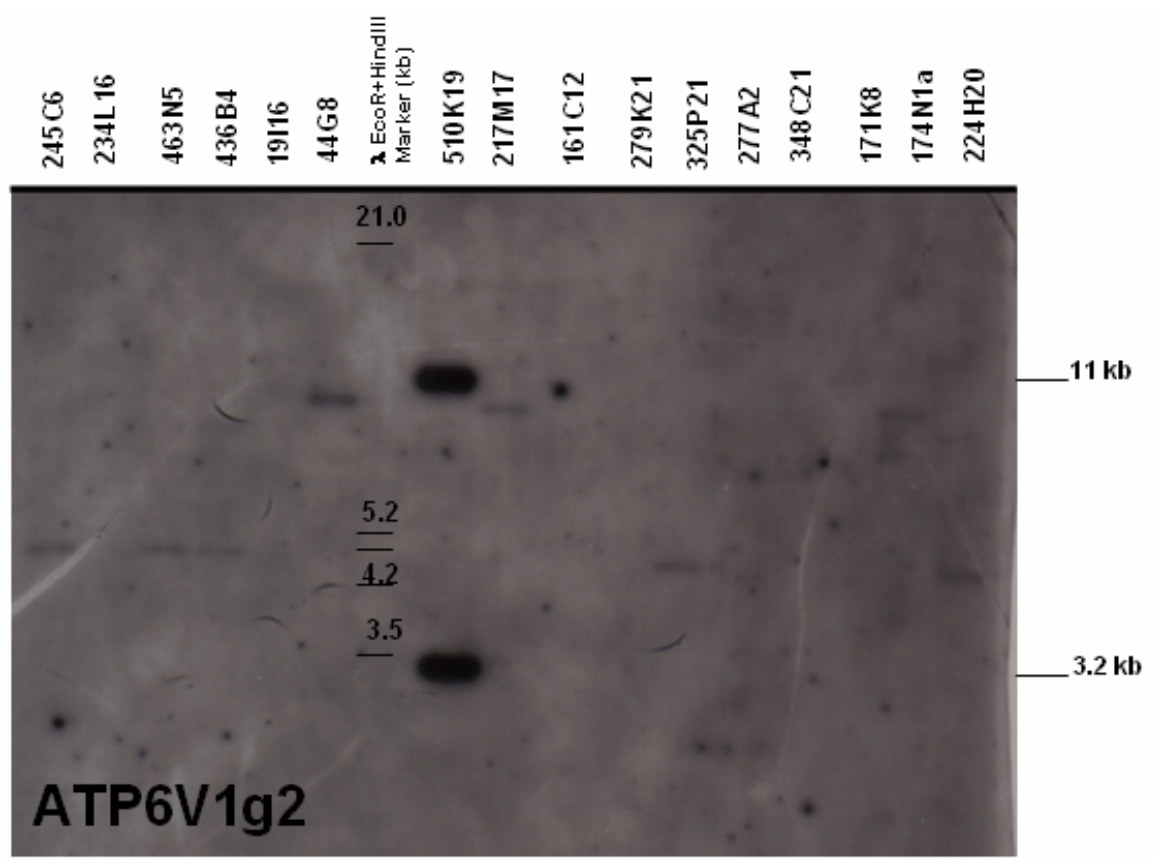

Figure 5.2.1.1.6: Autoradiograph after Southern blot hybridization of ATP6V1g2 framework gene probe with BAC-clone fragments (CHORI-259, Callithrix jacchus).

MHC-class I-positive BAC-clones were digested with restriction enzyme EcoRI. After gel electrophoresis and fingerprinting, their fragments were hybridized with said gene probe. DNA sample Lambda $(\lambda)$ EcoR/+HindIII used as ladder marker. kb values on the right margin calculated relative to marker DNA fragments which were seen by means of ethidium bromide fluorescence. 


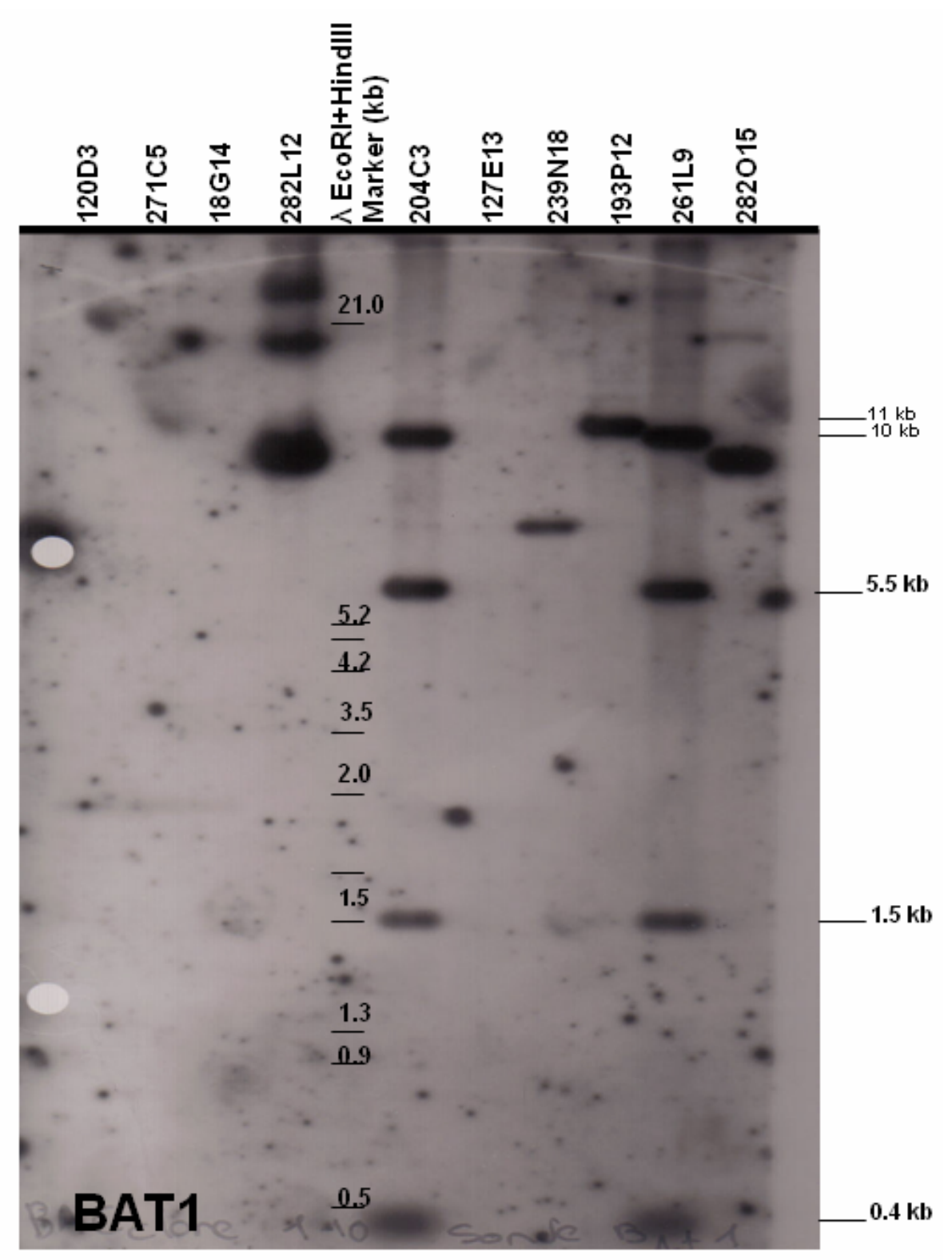

Figure 5.2.1.1.7: Autoradiograph after Southern blot hybridization of BAT1 framework gene probe with BAC-clone fragments (CHORI-259, Callithrix jacchus).

MHC-class I-positive BAC-clones were digested with restriction enzyme EcoRI. After gel electrophoresis and fingerprinting, their fragments were hybridized with said gene probe. DNA sample Lambda $(\lambda)$ EcoRI+HindIII used as ladder marker. kb values on the right margin calculated relative to marker DNA fragments which were seen by means of ethidium bromide fluorescence. 


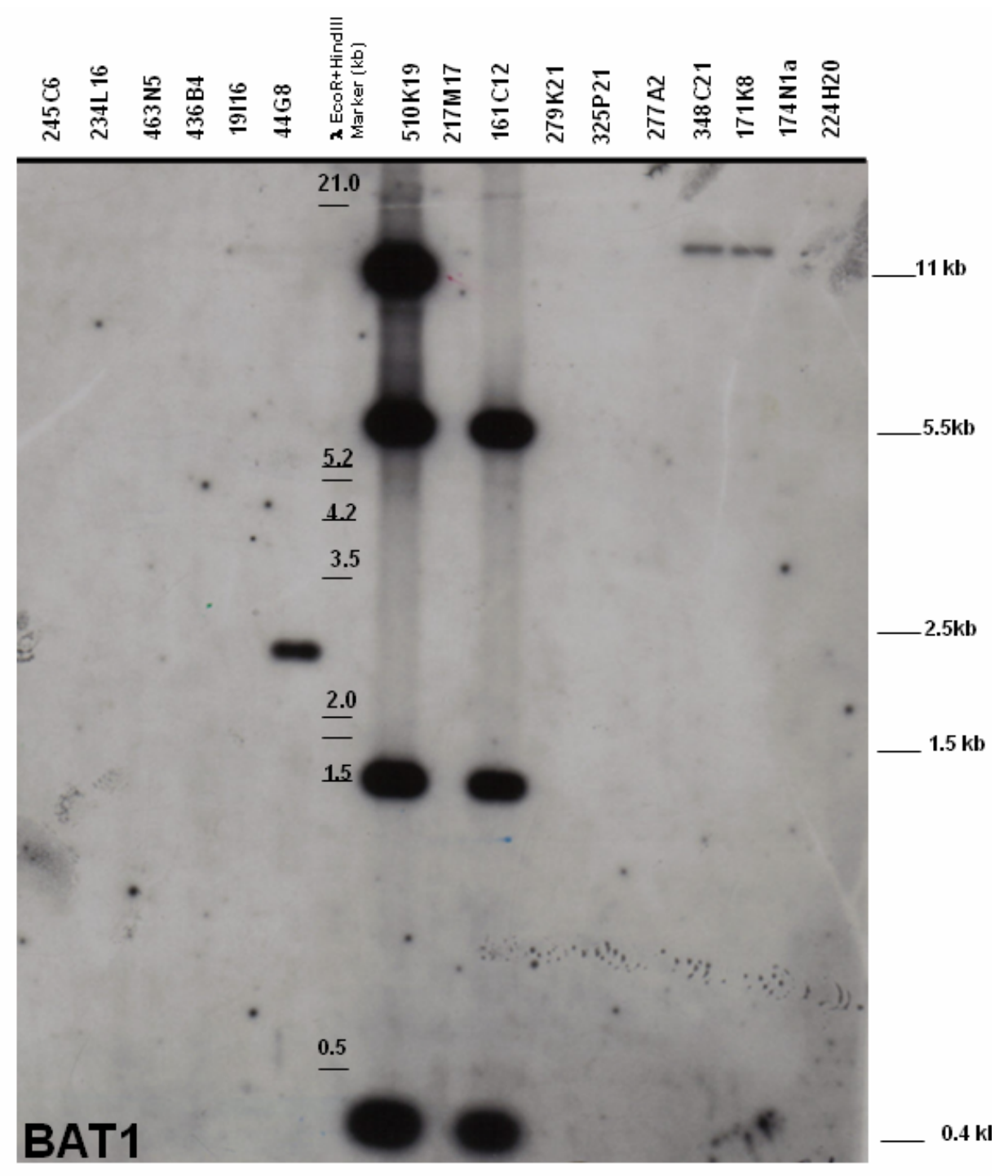

Figure 5.2.1.1.8: Autoradiograph after Southern blot hybridization of BAT1 framework gene probe with BAC-clone fragments (CHORI-259, Callithrix jacchus).

MHC-class I-positive BAC-clones were digested with restriction enzyme EcoRI. After gel electrophoresis and fingerprinting, their fragments were hybridized with said gene probe. DNA sample Lambda $(\lambda)$ EcoRI+HindIII used as ladder marker. kb values on the right margin calculated relative to marker DNA fragments which were seen by means of ethidium bromide fluorescence. 


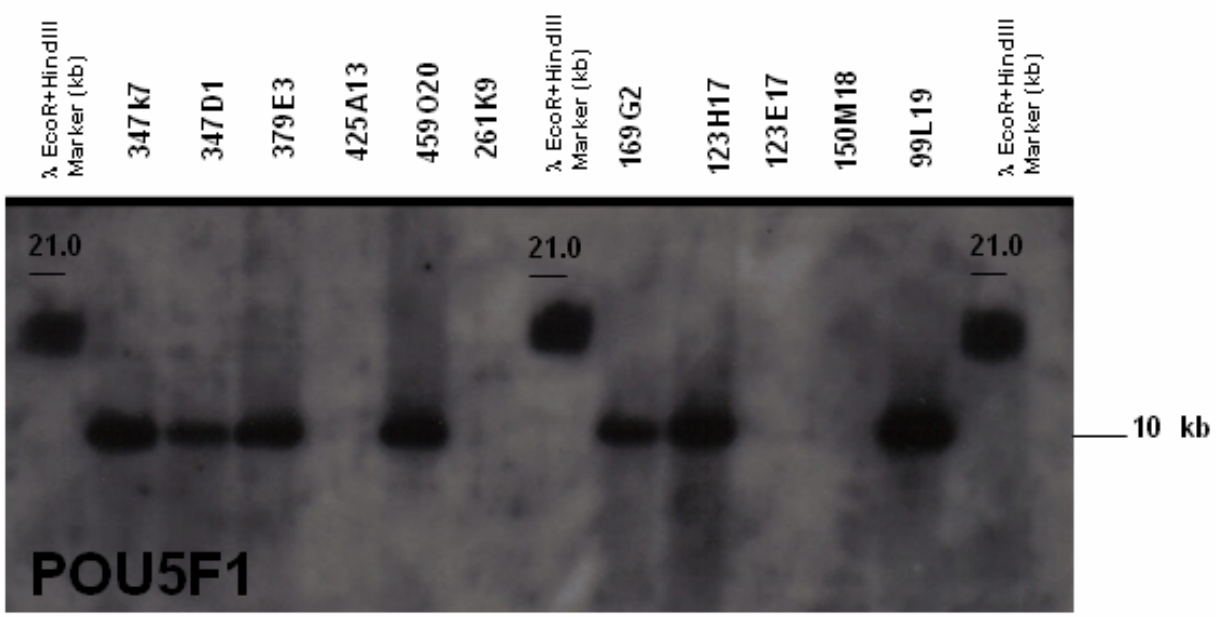

Figure 5.2.1.1.9: Autoradiograph after Southern blot hybridization of POU5F1 framework gene probe with BAC-clone fragments (CHORI-259, Callithrix jacchus).

MHC-class I-positive BAC-clones were digested with restriction enzyme EcoRI. After gel electrophoresis and fingerprinting, their fragments were hybridized with said gene probe. DNA sample Lambda $(\lambda)$ EcoRI+HindIII used as ladder marker. kb values on the right margin calculated relative to marker DNA fragments which were seen by means of ethidium bromide fluorescence. 

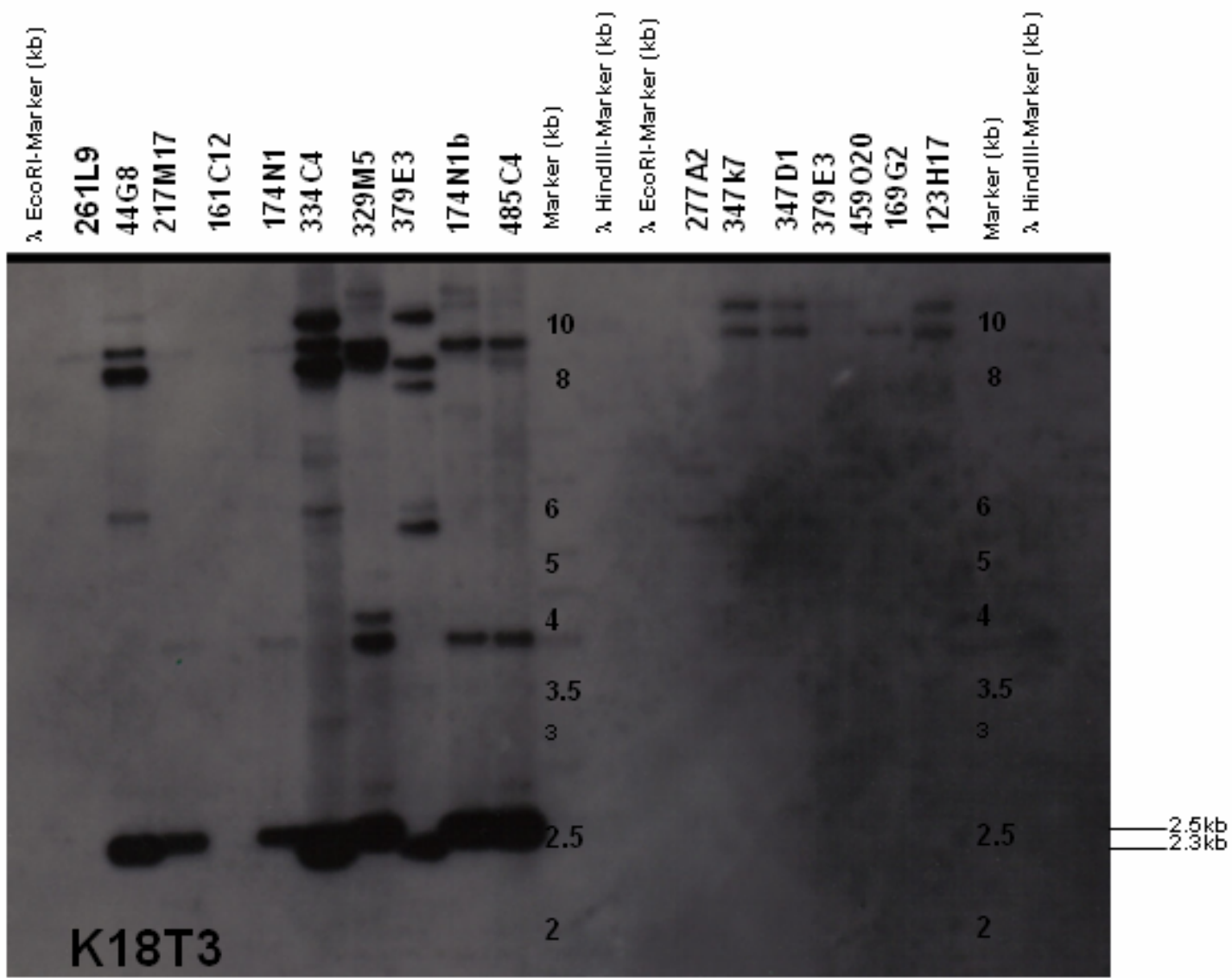

Figure 5.2.1.1.10: Autoradiograph after Southern blot hybridization of K18T3 probe with BAC-clone fragments (CHORI-259, Callithrix jacchus).

MHC-class I-positive BAC-clones were digested with restriction enzyme EcoRI. After gel electrophoresis and fingerprinting, their fragments were hybridized with said gene probe. DNA sample Lambda $(\lambda)$ EcoRI+HindIII used as ladder marker. kb values on the right margin calculated relative to marker DNA fragments which were seen by means of ethidium bromide fluorescence. 


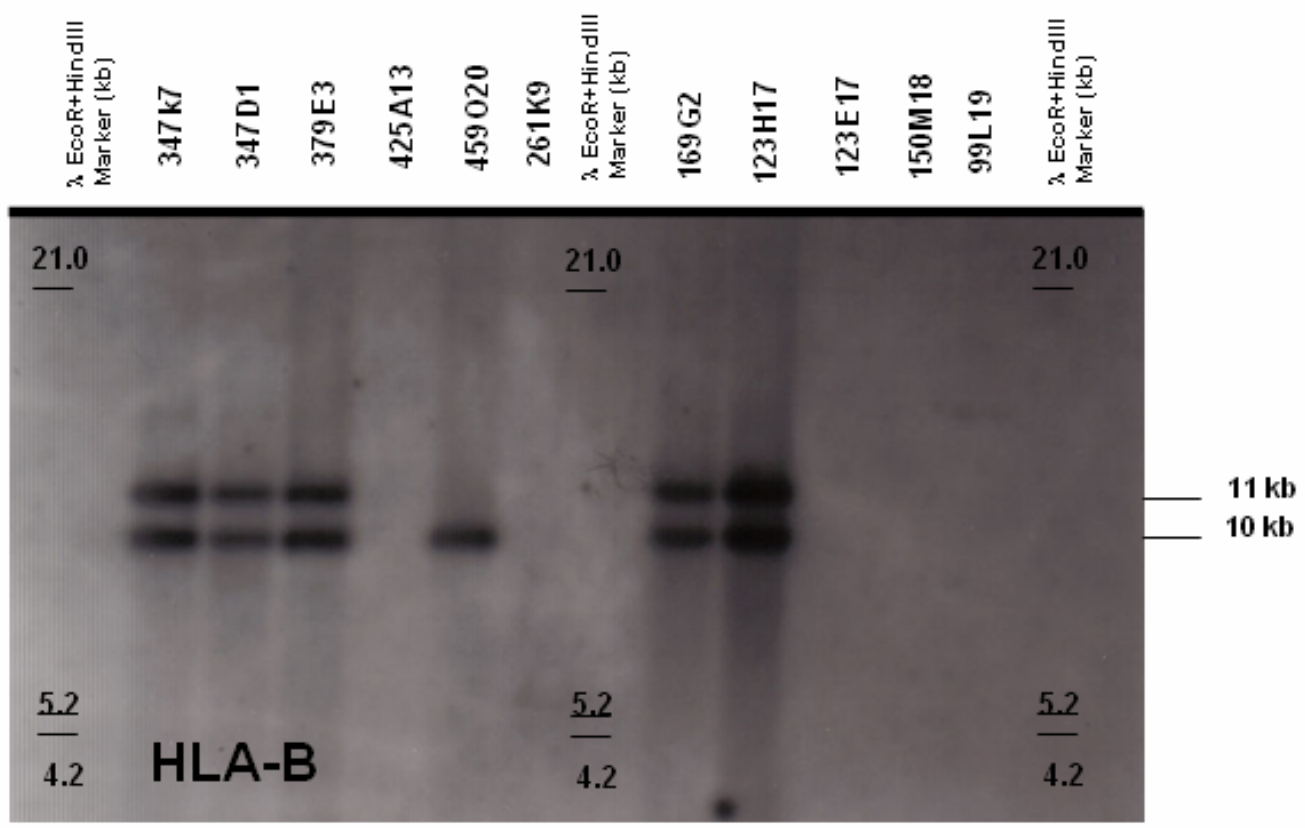

Figure 5.2.1.1.11: Autoradiograph after Southern blot hybridization of HLA-B probe with BAC-clone fragments (CHORI-259, Callithrix jacchus).

MHC-class I-positive BAC-clones were digested with restriction enzyme EcoRI. After gel electrophoresis and fingerprinting, their fragments were hybridized with said gene probe. DNA sample Lambda $(\lambda)$ EcoRI+HindIII used as ladder marker. kb values on the right margin calculated relative to marker DNA fragments which were seen by means of ethidium bromide fluorescence.

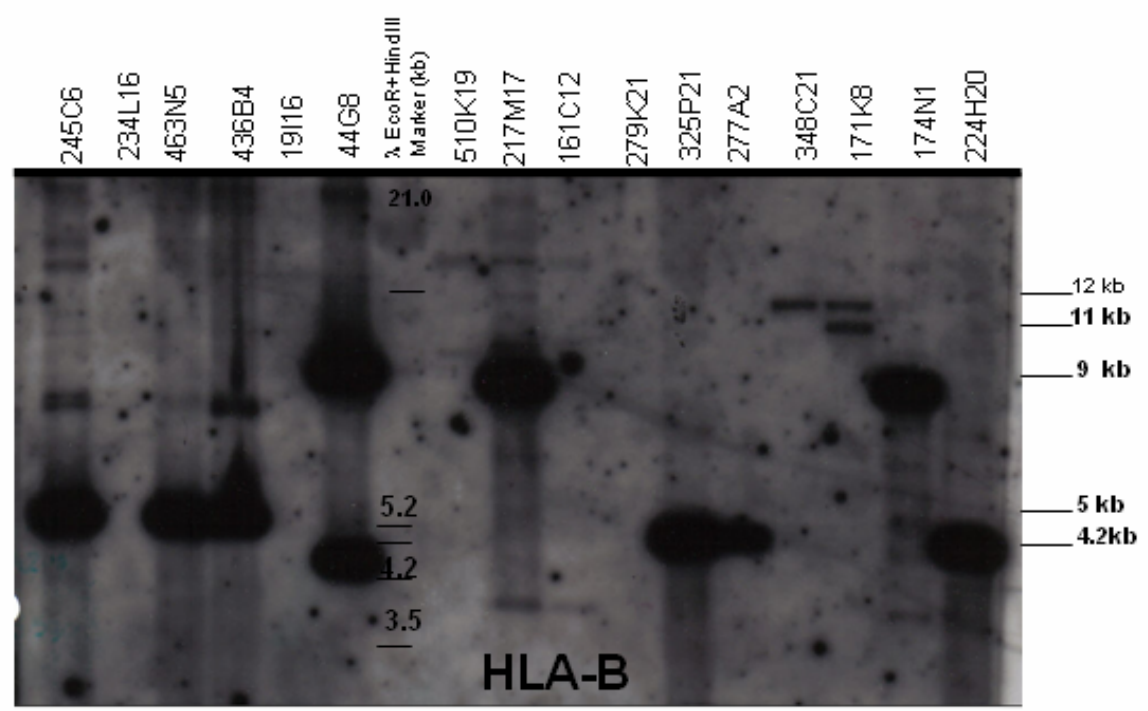


Figure 5.2.1.1.12: Autoradiograph after Southern blot hybridization of HLA-B probe with BAC-clone fragments (CHORI-259, Callithrix jacchus).

MHC-class I-positive BAC-clones were digested with restriction enzyme EcoRI. After gel electrophoresis and fingerprinting, their fragments were hybridized with said gene probe. DNA sample Lambda $(\lambda)$ EcoRI+HindIII used as ladder marker. kb values on the right margin calculated relative to marker DNA fragments which were seen by means of ethidium bromide fluorescence.

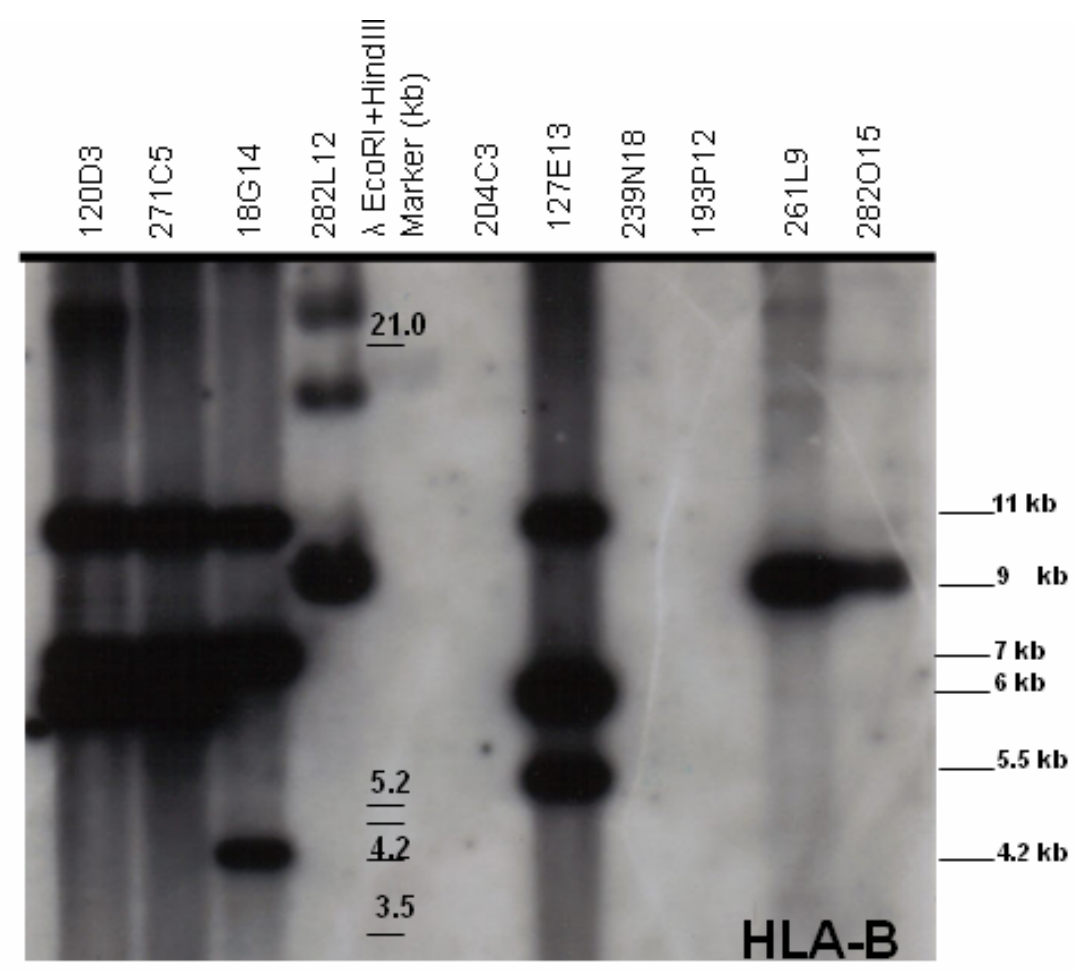

Figure 5.2.1.1.13: Autoradiograph after Southern blot hybridization of HLA-B probe with BAC-clone fragments (CHORI-259, Callithrix jacchus).

MHC-class I-positive BAC-clones were digested with restriction enzyme EcoRI. After gel electrophoresis and fingerprinting, their fragments were hybridized with said gene probe. DNA sample Lambda $(\lambda)$ EcoRI+HindIII used as ladder marker. kb values on the right margin calculated relative to marker DNA fragments which were seen by means of ethidium bromide fluorescence. 


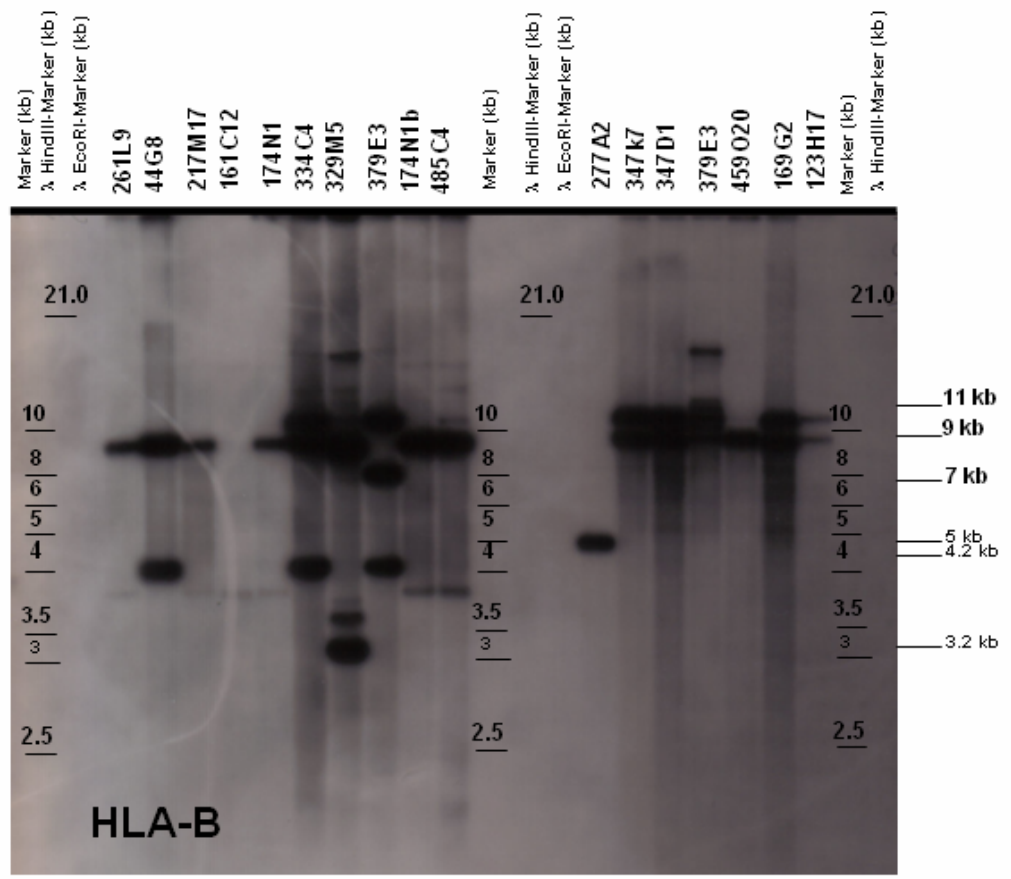

Figure 5.2.1.1.14: Autoradiograph after Southern blot hybridization of HLA-B probe with BAC-clone fragments (CHORI-259, Callithrix jacchus).

MHC-class I-positive BAC-clones were digested with restriction enzyme EcoRI. After gel electrophoresis and fingerprinting, their fragments were hybridized with said gene probe. DNA sample Lambda $(\lambda)$ EcoRI+HindIII used as ladder marker. kb values on the right margin calculated relative to marker DNA fragments which were seen by means of ethidium bromide fluorescence.

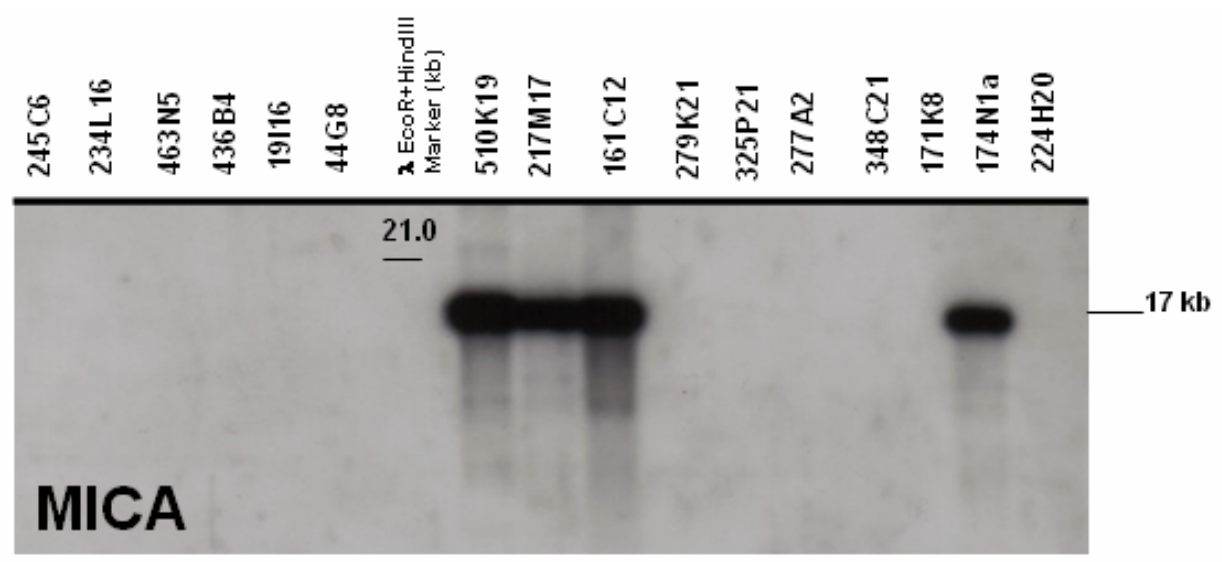

Figure 5.2.1.1.15: Autoradiograph after Southern blot hybridization of MICA probe with BAC-clone fragments (CHORI-259, Callithrix jacchus).

MHC-class I-positive BAC-clones were digested with restriction enzyme EcoRI. After gel electrophoresis and fingerprinting, their fragments were hybridized with said gene 
probe. DNA sample Lambda $(\lambda)$ EcoRI+HindIII used as ladder marker. kb values on the right margin calculated relative to marker DNA fragments which were seen by means of ethidium bromide fluorescence.

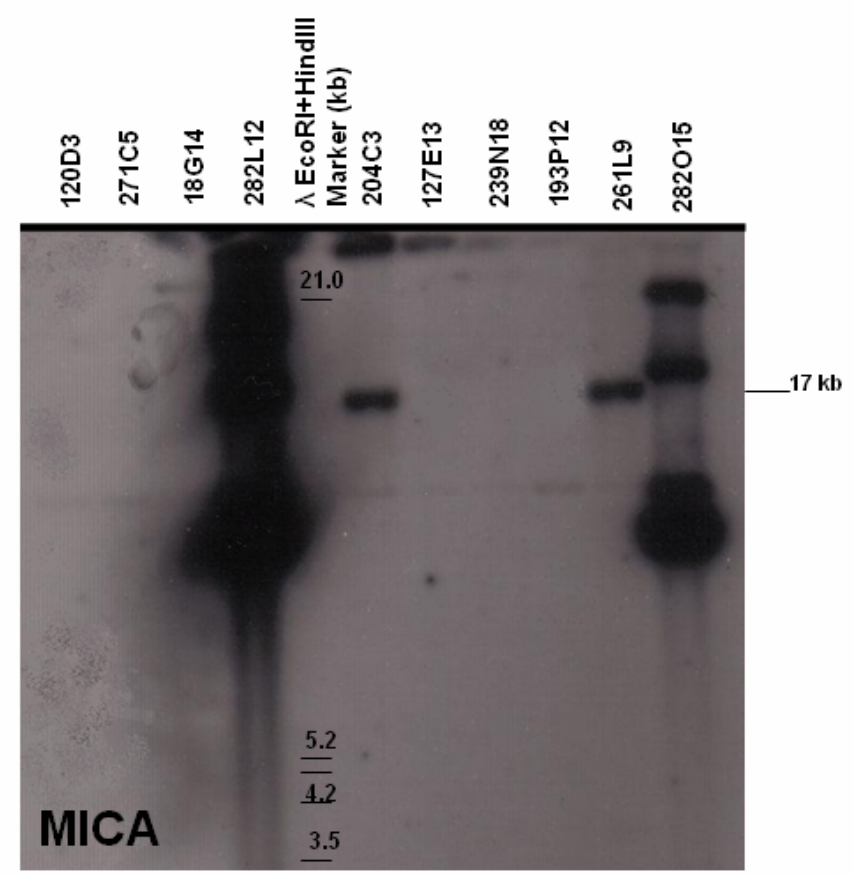

Figure 5.2.1.1.16: Autoradiograph after Southern blot hybridization of MICA probe with BAC-clone fragments (CHORI-259, Callithrix jacchus).

MHC-class I-positive BAC-clones were digested with restriction enzyme EcoRI. After gel electrophoresis and fingerprinting, their fragments were hybridized with said gene probe. DNA sample Lambda $(\lambda)$ EcoRI+HindIII used as ladder marker. kb values on the right margin calculated relative to marker DNA fragments which were seen by means of ethidium bromide fluorescence. 


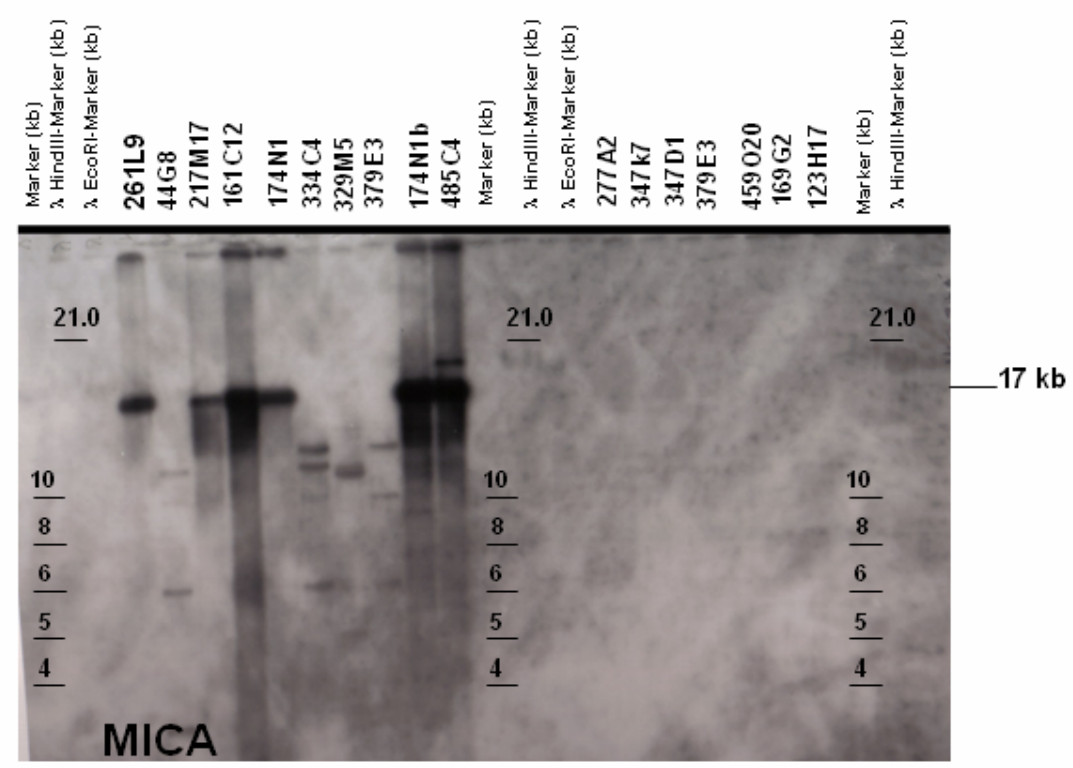

Figure 5.2.1.1.17: Autoradiograph after Southern blot hybridization of MICA probe with BAC-clone fragments (CHORI-259, Callithrix jacchus).

MHC-class I-positive BAC-clones were digested with restriction enzyme EcoRI. After gel electrophoresis and fingerprinting, their fragments were hybridized with said gene probe. DNA sample Lambda $(\lambda)$ EcoRI+HindIII used as ladder marker. kb values on the right margin calculated relative to marker DNA fragments which were seen by means of ethidium bromide fluorescence.

\subsubsection{Evaluation of hybridization results (contig 1)}

Nine of the 31 clones identified were used for did hybridize fragment analysis. 12 EcoR/ fragments of the nine clones (Table 5.2.1.1):

- MHC-class I-Caja-G: This probe found six fragments of $11 \mathrm{~kb}, 10 \mathrm{~kb}, 9 \mathrm{~kb}, 7$ $\mathrm{kb}, 4.2 \mathrm{~kb}$ and $3.5 \mathrm{~kb}$. These 15 clones appeared to be overlapping: 261L9, 44G8, 174N1, 217M17, 174N1, 485C4, 334C4, 329M5, 379E3, 347K7, 347D1, 379F14, 123H17, 169G2 and 459O20 (Figures 5.2.1.1.1- 5.2.1.1.4, respectively).

- ATP6Vg2: This framework gene probe found two fragments of $11 \mathrm{~kb}$ and 3.2 kb. Three clones 204C3, 510K19 and 261L9 appeared to be overlapping (Figures 5.2.1.1.5 and 5.2.1.1.6, respectively).

- BAT1: This framework gene probe found four fragments, of $11 \mathrm{~kb}, 5.5 \mathrm{~kb}, 1.5$ $\mathrm{kb}$ and $0.4 \mathrm{~kb}$. Four clones appeared to be overlapping: 204C3, 510K19, 
261L9, and 161C12. The clone 161C12 was cleaved into the fragments 5.5 $\mathrm{kb}, 1.5 \mathrm{~kb}$ and $0.4 \mathrm{~kb}$ only (Figures 5.2.1.1.7 and 5.2.1.1.8, respectively).

- POU5F1: This framework gene probe found one fragment of $10 \mathrm{~kb}$. These six clones appeared to be overlapping: 347K7, 379F14, 459O20 123H17, 169G2, and 99L19 (Figure 5.2.1.1.9).

- K18T3: This probe found two fragments of $2.5 \mathrm{~kb}$ and $2.3 \mathrm{~kb}$. These eight clones appeared to be overlapping: 44G8, 174N1, 217M17, 174N1, 485C4, 334C4, 329M5 and 379E3 (Figure 5.2.1.1.10).

- MHC-class I-HLA-B: This probe found six fragments, the same as found by the MHC-class I-probe plus one fragment of $3.2 \mathrm{~kb}$. These 15 clones appeared to be overlapping: 261L9, 44G8, 174N1, 217M17, 174N1, 485C4, 334C4, 329M5, 379E3, 347K7, 347D1, 379F14, 123H17, 169G2 and 459O20 (Figures 5.2.1.1.11 - 5.2.1.1.14, respectively).

- MICA: This gene probe found one fragment of $17 \mathrm{~kb}$. The following seven clones appeared to be overlapping: 510K19, 204C3, 261L9, 161C12, 174N1, 217M17 and 485C4 (Figures 5.2.1.1.15 - 5.2.1.1.17, respectively).

- TCF19: This framework gene probe was negative in hybridization with the fragments of all the BAC clones checked.

In respect to the organization of framework genes, MHC-class I, HLA-B and MICA hybridization of fragments was done to achieve a schematic representation of contig 1. Contig 1 appears to be represented by the following 19 BAC clones: 204C3, 510K19, 261L9, 161C12, 44G8, 174N1, 217M17, 174N1, 485C4, 334C4, 329M5, 379E3, 347K7, 347D1, 379F14, 123H17, 169G2, 459O20 and 99L19 (Figure 5.2.18). Screening of BAC bank was done with a MIC-A probe from rhesus monkey. Results are in agreement with those obtained in rhesus monkey and human for contig 1; however, the other contigs were not found MICA positive, in contrast to findings in the intervals GNL1 - TRIM39 (with MICC) and TCTEX 4 - MOG (with MIC D,G, and F) in rhesus monkey and human (The MHC sequencing consortium 1999; Seo et al., 2001), chimpanzee (Anzai et al., 2003) and Microcebus murinus (Neff, 2005), see MHC class I map, Figure 5.1.1.

Both MIC genes A and B may be assigned to five BAC clones of Callithrix jacchus as the band $17 \mathrm{~kb}$ separated by electrophoresis may well contain two co-migrating fragments of similar length. This interpretation shown in Figure 5.2.2 is supported by 
two corresponding long EcoRl fragments in Hs. In the ethidiumbromide stained electropherograms, larger relative amounts of DNA can be seen for the clones K17 and 19 as compared to $\mathrm{k} 18,25,41$ and 42 . For publication, densitometry should confirm these visual results.

The interval between BAT1 and TCF19 is polymorphic; polymorphic applies to regions with genetic alterations between species compared in respect to the MHC class I region, HLA-B, HLA-C and MIC genes in several mammalian species. A double positive screening of BAC clones was included with $M H C$ class I gene Caja-G and the following probes: ATP6V1g2, BAT1, TCF19, HLA-B, POU5F1, MICA and K18T3.

The clones 510K19, 204C3 and 261L9 were overlapping in ATP6V1g2 Eco-RI fragments $3.2 \mathrm{~kb}$ and $11 \mathrm{~kb}$, this framework is localized next to BAT1. The clones 510K19, 204C3, 261L9 and 161C12 were overlapping in BAT1 with the EcoRI fragments $0.4 \mathrm{~kb}, 1.5 \mathrm{~kb}, 5.5 \mathrm{~kb}$, and $11 \mathrm{~kb}$ (in clone 44G8 found only $2.5 \mathrm{~kb}$ ); however clone 239N19 had a fragment $8 \mathrm{~kb}$ and clone193P12 had $12 \mathrm{~kb}$. Both clones were not included in contig 1 because uncertainty of their potential overlapping with BAT1 and MHC class I genes (Figure 5.2.1.3).

From the BAC clones screening with BAT1, TCF19 and MHC class I probes, respectively, 31 were selected for characterization of their fragments by Southern blot fingerprint and sequence analysis (Table 5.2.1.2.1).

Table 5.2.1.2.1: Eco-RI fragments of Hs MHC class I contigs:

Data used for constructing Callithrix jacchus gene maps (Figure 5.4.1). T39= TRIM39, T26=TRIM26, T5= TCTEX5, T4= TCTEX4, M= MOG, P= POU5F1 and ATP $=$ ATP6Vg1, see next page. 


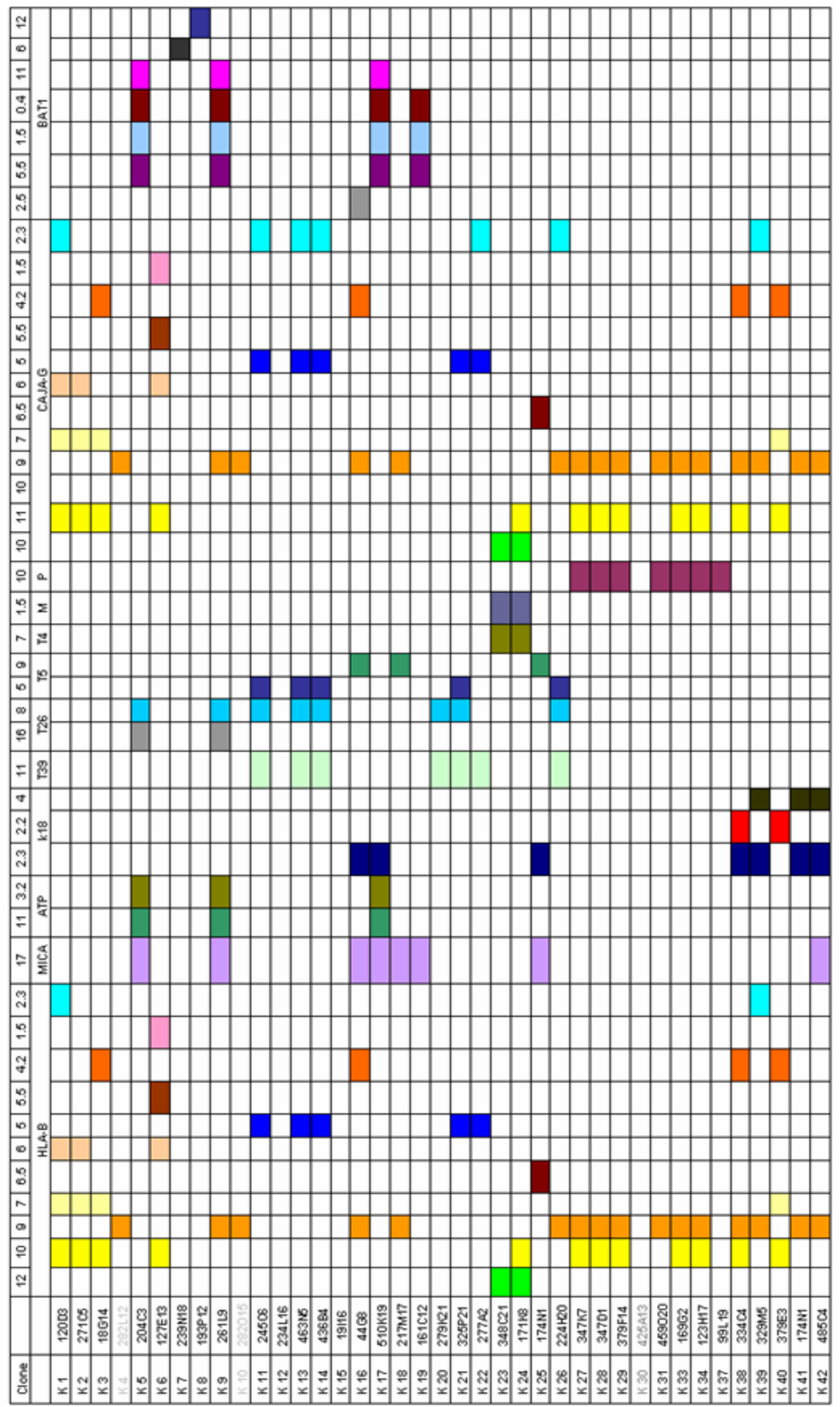




\subsubsection{Construction of a contig 1 map}

A gene map of contigs in Callithrix jacchus was attempted using these hybridization results. Unfortunately, even in contig 1 - with its biggest share of clones selected -, the amount of information - for reasons discussed - turned out to be too small for constructing a consistent contig of BAC clone inserts. In view of the redundancy (clustering) of similar genes in this region, all hybridizing nominally different class I probes alike, fragment-probe hybridization assignment as usually done for using just their information for mapping requires a sequence of shorter and more fragments all having different lengths and distinctively defined gene contents of individual genes. Fortunately, a second source of information was found that could help out in a comparative interpretation of the data: the well-documented human MHC class I DNA with its many known gene loci. The computer was made to do an EcoRI splitting of the Hs contig 1 DNA and to deliver a proper long list of all 123 fragment lengths in bp, furthermore, an assignment of all genes known for this section, and even the definitions of both fragment and gene loci by their 1st and last bp numbers (according to both, Shiina's and Venter's bp scales).

With this ancillary, comparative information, at least 14 genes of contig 1 as written in the headline of figure 5.2.1.3.1 could be identified which by their order and fragment length(s) did allow the arrangement of all the BAC clone fragments obtained, according to their probe specifications, into the gene map shown in Figure 5.2.1.3.1.

Consequently, a minimal contig of clones for an eventual sequencing of contig 1 could be composed of clones 510K19, 334C4, 120D3 and e.g. 347K7. Their inserts, taken together, should be covering almost the entire contig 1, i.e. $418 \mathrm{~kb}$ or more in Hs. A table of the EcoRI fragments in Hs, generally fitting those found in Callithrix jacchus, plus a map that illustrates the locations and lengths of both fragments and genes assigned shall be given in a subsequent publication. It suggests great similarity of EcoRI splitting in Hs and Callithrix; differences in a few cleavage sites are suggested, however, by some of the hybridization results. A map of this kind is a prediction having no absolute certainty. Of preliminary nature, it will be confirmed or corrected by the DNA sequencing that it shall support. 


\section{Contig 1 genes}

Fragments in $\mathbf{k b}$$$
3.2 \quad 11=1
$$$$
1.5 \quad 0.4
$$

HLA-X MICA + HLA-B

$9 ?$

$172.3+6.5=8.82 .34 .2$

BAC clones

$\mathrm{K} 5: 204 \mathrm{C3}$

$\begin{array}{llllllll}3.2 & 11=11 & 1.5 & 0.4 & 5.5 & 17 & 8 & 17\end{array}$

K9:261L9

$\begin{array}{lllllllll}3.2 & 11=11 & 1.5 & 0.4 & 5.5 & 17 & 8 & 17 & 9 ?\end{array}$

K17:510K19

\begin{tabular}{llllllllll}
3.2 & $11=11$ & 1.5 & 0.4 & 5.5 & 17 & 8 & 17 & 9 & 2.3 \\
\hline
\end{tabular}

K19:161C12

\begin{tabular}{llllllllll}
1.5 & 0.4 & 5.5 & 17 & 8 & 17 & $?$ & $\mathbf{2 . 3}$ & & \\
\hline & & & & & & & & & \\
& 2.5 & 17 & 8 & 17 & 9 & $\mathbf{2 . 3}$ & $\boldsymbol{?}$ & 4.2
\end{tabular}

K16:44G8

\begin{tabular}{llll}
6.5 & 17 & 9 & 2.3 \\
\hline
\end{tabular}

K25:174N1a

\begin{tabular}{lll}
17 & 9 & 2.3 \\
\hline
\end{tabular}

K42:485C4

$\begin{array}{llll}17 & 9 & 2.3 & 4.2 \\ & & & \\ 17 & 9 & 2.3 & 4.2\end{array}$

K41:174N1b

K39:329M5

K38:334C4

K40:379 E3

K3:18G14

K6:127E13

K2:271C5

K1:120D3

\begin{tabular}{lllll}
17 & 9 & 2.3 & 4.2 & \\
\hline & & & & \\
& 9 & 2.3 & 4.2 & 2.3 \\
\hline
\end{tabular}

\begin{tabular}{lllll}
9 & 2.3 & 4.2 & $?$ & 11 \\
\hline
\end{tabular}

\begin{tabular}{rrrr}
2.2 & 7 & 11 & 4.2 \\
\hline 7 & 11 & 4.2 \\
\hline
\end{tabular}

\begin{tabular}{llll}
5.5 & 11 & 6 & 1.5 \\
\hline
\end{tabular}

\begin{tabular}{lll}
7 & 11 & 6 \\
\hline
\end{tabular}

\begin{tabular}{llll}
7 & 11 & 6 & 2.3 \\
\hline
\end{tabular}

K27:347 K7, K28:347 D1, K29:379 F14, K31:459 020, K33:169 G2 and K34:123 H17 equally show

$11 ?$

?

$1110=10$ 
Several months after finishing this map, we have learnt from an e-mail by Prof. T. Shiina the reason why comparison of these Caja fragments with those of Hs is rather inadequate: the contig 1 or HLA-B/C sections of the two primates are too different in their organization that is complicated in Caja by the existence of $10 \mathrm{~B} / \mathrm{C}$ duplicons compared to $1 \mathrm{~B}$ and $1 \mathrm{C}$ duplicon in Hs - expanding contig 1 from 418 to some 600 $\mathrm{kb}$. Yet, gene order on both sides of the duplicons might still be quite alike.

Between the frameworks genes BAT1 and POU5F1-TCF19 have been localized contig 1 as part of the MHC class I region, in humans with a length of $418 \mathrm{~kb}$ (The MHC consortium, 1999), as H2-complexes in mouse with $300 \mathrm{~kb}$, as RT1-complex of rat with $450 \mathrm{~kb}$ (Hurt, et al., 2004) and of chimpanzee (Patr) (Fukami-Kobayashi et al., 2005). These framework genes were found to be orthologoues. In humans this interval contains the MICA and MICB genes (Bahram et al., 1994), HLA-B and HLAC. The MHC class I genes homologoues to HLA-B as expressed in this New World primate are not orthologous to any of the classical MHC class I loci of the Catarrhini $(A, B$, or $C$ loci); instead, they are most similar to the human non-classical $H L A-G$ (Watkins et al., 1990).

In conclusion, the contig 1 has been found between BAT1 and POU5F1 by fragments and specific hybridizations of probes Caja-G, MICA, Mamu-B and respective framework genes (including ATP6Vg1 and TCF19) display an order of genes not different from humans and other primates.

The restriction map obtained here suggests locations of these Caja genes very similar to the human ones. The number of class I genes in contig 1 of Callithrix presumably equals that in Hs, where 14 are known so far: three HLA genes + nine pseudogenes + two MIC genes (cf. class I map, Figure 5.2.1.3.1). Of these, the map obtained displays evidence for nine or perhaps ten. A further gene, next to POU5F1, called HCGII-11 is predicted to possibly explain the observation that six of the BAC clones rendered a fragment of approximately $10 \mathrm{~kb}$ that hybridizes with POU5F1, Caja-G and HLA-B (see Table 5.2.1.2.2). It shall be searched for its eventual alignment and be commented in the sequences chapter 5.5.1. 


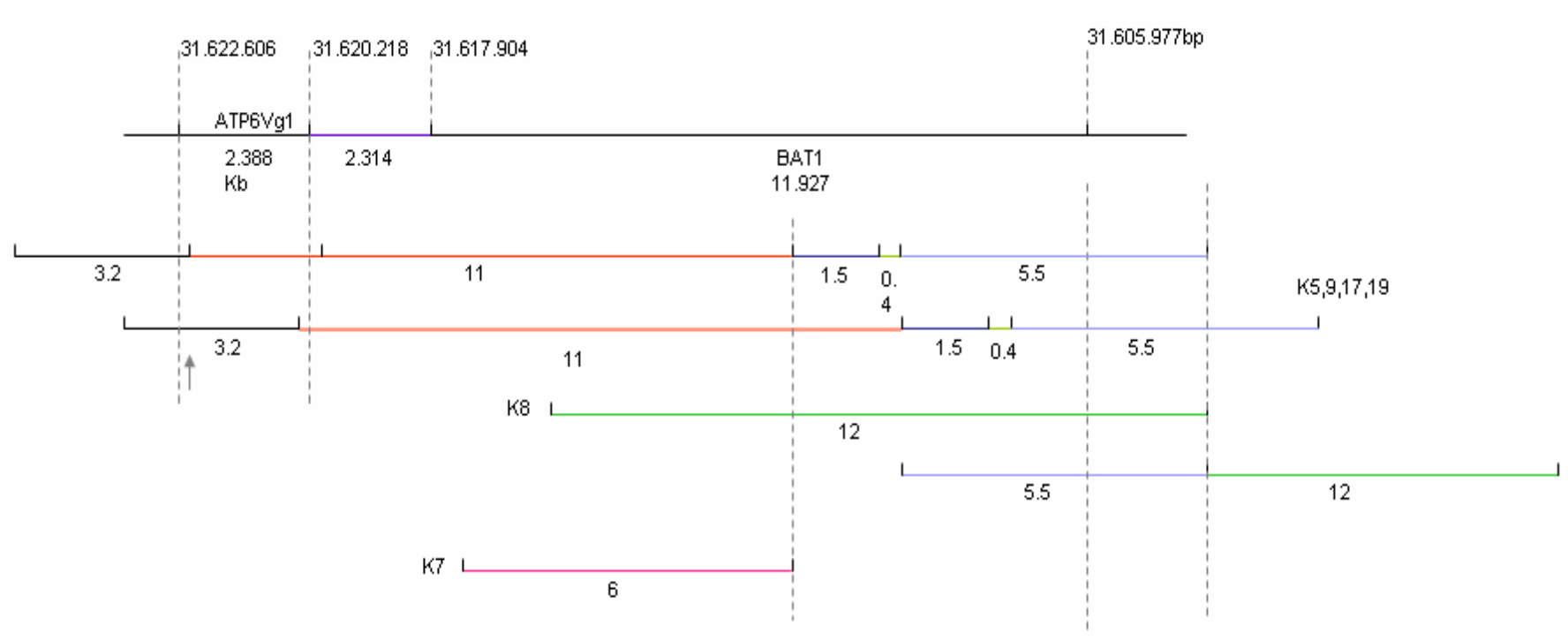

Figure 5.2.1.3.2: Alignment of BAC-clone insert fragments to ATP6Vg1 and BAT1 to the Hs bp scale by Shina ID:BA000025.

All four BAT1 postive fragments, of 11, 1.5, 0.4 and $5.5 \mathrm{~kb}$ from clones $\mathrm{k} 5, \mathrm{k}$ 9, k17 and k19 fit in as shown. Fragments from clones k8, (12kb) and K7 (6kb) are neither helpful nor against the map. Possibly these clones are EcoRI cleaved in a modified way.

Another cross reaction is seen in a fragment of $11 \mathrm{~kb}$ that hybridizes with the neighbored genes ATP6Vg1 and BAT1. This fragment may bridge an intergenic gap as shown graphically in figure 5.2.1.3.2. In the endeavor of explaining the genetic data obtained for Callithrix during the experimental part of this work, thinking in terms of genomic lengths was needed. It led to the construction of a map to bp-scale for the entire $\mathrm{MHC}$ class I region in $\mathrm{Hs}$, the primate chosen for reference (5.2.1.3.1).

\subsubsection{Contig 3, the interval TRIM26 - TRIM39}

\subsubsection{Southern blot analysis of contig 3}

Southern blot analysis of BAC clones for screening with framework probes of MHC class I region, Caja-G, HLA-B, TRIM26, TRIM39 and TCTEX5, included seven clones belonging to contigs as defined here in analogy to the human MHC class I contig 3 (245C6, 463N5, 436B4, 277A2, 224H20, 325P21 and 279K21). This contig presented also two class I positive EcoR-I fragments, of $2.5 \mathrm{~kb}$ and $5 \mathrm{~kb}$. The $2.3 \mathrm{~kb}$ fragments hybridized with both probes, Caja- $\mathrm{G}$ and Mamu-B, the $5 \mathrm{~kb}$ fragment only with Caja-G. This discrepancy seems unimportant, but usually, these two probes 
hybridized equally. Clone 325P21, however, rendered only the fragment of $5 \mathrm{~kb}$. This indicates presence of MHC class I gene(s) in or near the interval TRIM26 - TRIM39 had run observed in rat, mouse, chimpanzee and human by Hurt et al., 2004; Anzai et al., 2003; Reymond, 2001; Wheeler et al., 2001; Orimo et al., 2000; Rahman, 1998; Yoshino et al., 1998; Chu et al., 1995 and Wei, (unpublished).

Callithrix jacchus did not show MICA positive EcoRI fragments as have also not been found in human (Bahram et al., 1994). The MIC genes expected in contigs 3 and 4 are pseudogenes. (see Figure 5.1.1).

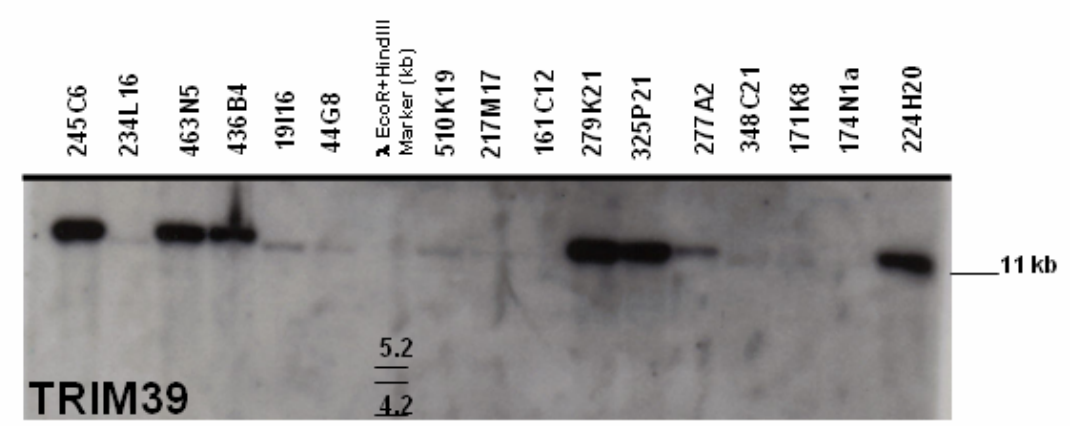

Figure 5.2.2.1.1: Autoradiograph after Southern blot hybridization of TRIM39 framework gene probe with BAC-clone fragments (CHORI-259, Callithrix jacchus).

MHC-class I-positive BAC-clones were digested with restriction enzyme EcoRI. After gel electrophoresis and fingerprinting, their fragments were hybridized with said gene probe. DNA sample Lambda $(\lambda)$ EcoR/+HindIII used as ladder marker. kb values on the right margin calculated relative to marker DNA fragments which were seen by means of ethidium bromide fluorescence. 


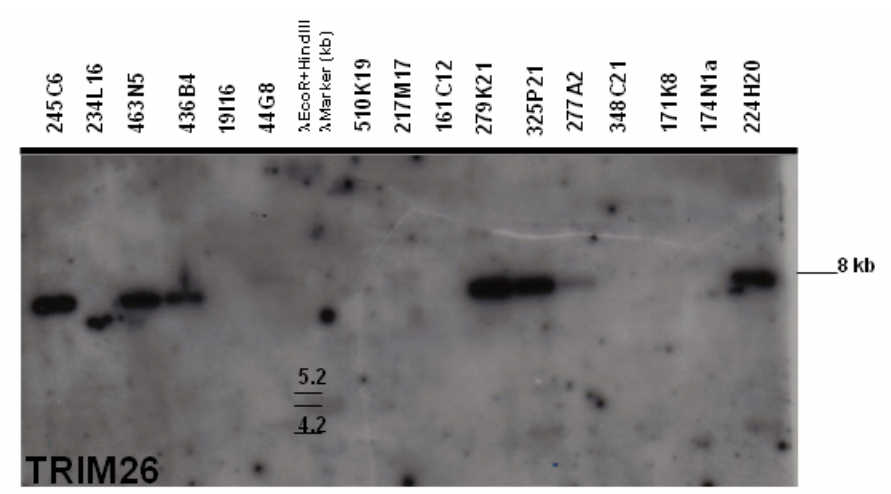

Figure 5.2.2.1.2: Autoradiograph after Southern blot hybridization of TRIM26 framework gene probe with BAC-clone fragments (CHORI-259, Callithrix jacchus).

MHC-class I-positive BAC-clones were digested with restriction enzyme EcoRI. After gel electrophoresis and fingerprinting, their fragments were hybridized with said gene probe. DNA sample Lambda $(\lambda)$ EcoRI+HindIII used as ladder marker. kb values on the right margin calculated relative to marker DNA fragments which were seen by means of ethidium bromide fluorescence.

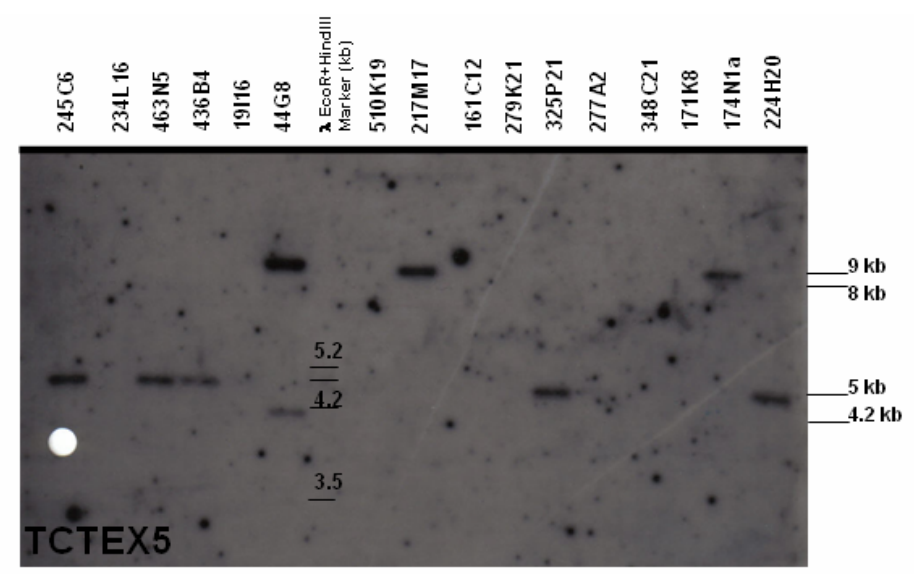

Figure 5.2.2.1.3: Autoradiograph after Southern blot hybridization of TCTEX5 framework gene probe with BAC-clone fragments (CHORI-259, Callithrix jacchus).

MHC-class I-positive BAC-clones were digested with restriction enzyme EcoRI. After gel electrophoresis and fingerprinting, their fragments were hybridized with said gene probe. DNA sample Lambda $(\lambda)$ EcoRI+HindIII used as ladder marker. kb values on the right margin calculated relative to marker DNA fragments which were seen by means of ethidium bromide fluorescence. 


\subsubsection{Evaluation of hybridization results (contig 3)}

From seven BAC clones of the Callithrix jacchus library, a total of 10 ECoRI (insert) fragments were obtained and used for screening of all BAC clones in the library:

- TRIM39: This framework gene probe detected one fragment of $11 \mathrm{~kb}$. These clones appeared to be overlapping: 245C6, 463N5, 436B4, 279K21, 325P21, 277A2, 503C3. The clones 234L16, 19I16, 44G8, 510K9, 217M17, 348C21, $171 \mathrm{~K} 8$ and $174 \mathrm{~N} 1$ were negative (Figure 5.2.2.1.1).

- TRIM26: This framework gene probe detected one fragment of $8 \mathrm{~kb}$. These clones appeared to be overlapping: 245C6, 463N5, 436B4, 279K21, 325P21, 277A2 and 503C3. The clones 234L16, 19I16, 348C21, 171K8, and 174N1 were negative (Figure 5.2.2.1.2).

- TCTEX5: This framework gene probe detected one fragment of $5 \mathrm{~kb}$. These clones appeared to be overlapping: 245C6, 463N5, 436B4, 325P21, 503C3, the clone 44G8, however presented two fragments $9 \mathrm{~kb}$ and $4.2 \mathrm{~kb}$, and the clones $217 \mathrm{M} 17$ and $174 \mathrm{~N} 1$ found one fragment: $8 \mathrm{~kb}$ (Figure 5.2.2.1.3).

- CAT56: This framework gene probe was negative in hybridization for each of the clones checked.

- MHC class I I-Caja-G: This probe detected two fragments of $2.5 \mathrm{~kb}$ and of 5 kb. These clones appeared to be overlapping: 245C6, 463N5, 436B4, 325P21, 277A2 and 503C3, where as clone 279K21 had only the $4.2 \mathrm{~kb}$ fragment (Figure 5.2.1.1.1).

- MHC-class I-HLA-B: This probe found one fragment of $5 \mathrm{~kb}$. These clones appeared to be overlapping: 245C6, 463N5, 436B4, 325P21, 277A2, 503C3, but the clone $279 \mathrm{~K} 21$ was negative (Figure 5.2.1.1.12).

- MICA: This probe was negative in the intervals 2 and 3 for each of the seven clones tested (Figure 5.2.1.1.15).

\subsubsection{Contribution to a contig 3 map}

The intervals CAT56 - TRIM39 and TRIM39 - TRIM26 of the MHC class I region have polymorphic margins in humans. They both belong to contig 3 in the human, where contig 2 is the longest of the four contigs, however almost devoid of class I genes (see map, Figure 5.1.1). The small numbers of clones investigated in this area did not really allow constructing any one of the contigs 2 - 4, i.e. a share of stepwise overlapping clone inserts with sufficiently many, short and well discernible fragments 
covering the segment between its framework borders. The intervalls expected by comparison with Hs and other mammals seem to be existing, however, as evidenced by positive screening of clones with the probes Caja-G, CAT56, TRIM39 and TRIM26 (see Figure 5.2.2.1.1 and 5.2.2.1.2). Of course, their existence in Callithrix jacchus is proven quite clearly by sequences done and localized as seen in map, figure 5.4.1. These sequences confirm 30 different genes and pseudogenes of the Hs contig 4, several of contig 3 and 2, many of contig 1.

The Interval CAT56 - TRIM39 contains class I gene(s) between the conserved framework genes CAT56 and TRIM39, with approximately of $500 \mathrm{~kb}$ in case of the RT1-complex of rat (Hurt et al., 2004).

The second intervall contains class I gene(s) between the conserved framework genes TRIM39 and TRIM26 with approximately of $200 \mathrm{~kb}$ in the RT1-complex. Near TRIM26, towards contig 4, are TRIM15 and TRIM10 (Hurt, et al., 2004). For practical reasons, the framework gene TCTEX5 located near TCTEX4 within less than $50 \mathrm{~kb}$, was included in contig 4 presumed by comparison. TCTEX5 considered as end of a contig 3 detected clones with the framework genes TRIM 26 and TRIM39. So, there is probably no gap in Callithrix between its presumable contigs 3 and 4 (Hurt et al., 2004; Jones et al., 1999; Zhang et al., 1998; Amadou et al., 1999).

Arrangement of framework genes, Caja-G and HLA-B studied by hybridization of fragments is shown in figure 5.2.1.1.1 and table 5.2.1.1.12, the order being given by homology with other mammals. 


\section{Contig 3}

\begin{tabular}{|c|c|c|c|c|c|}
\hline & \multicolumn{2}{|c|}{$\begin{array}{l}\text { HLA-E } \\
\text { (Caja-G) }\end{array}$} & \multirow{2}{*}{$\begin{array}{l}\text { TRIM } 39 \\
11\end{array}$} & \multirow{2}{*}{$\begin{array}{c}\text { TRIM26 } \\
8\end{array}$} & \multirow{2}{*}{$\begin{array}{c}\text { TCTEX } 5 \\
5\end{array}$} \\
\hline & 2.3 & 5 & & & \\
\hline K11:245C6 & 2.3 & +5 & 11 & 8 & 5 \\
\hline K13:463N5 & 2.3 & +5 & 11 & 8 & 5 \\
\hline K14:436B4 & 2.3 & +5 & 11 & 8 & 5 \\
\hline $\mathrm{K} 26: 224 \mathrm{H} 20$ & 2.3 & $\begin{array}{l}+5 \\
\end{array}$ & 11 & 8 & 5 \\
\hline K22:277 A2 & $\underline{2.3}$ & +5 & 11 & $?$ & \\
\hline K21:325P21 & & 5 & 11 & 8 & $5 ?$ \\
\hline$K 20: 279 k 21$ & & & 11 & 8 & \\
\hline
\end{tabular}

Figure 5.2.2.3: The overlapping BAC clone fragments drawn according to the order of framework genes known for contig 3 (intervals CAT56 - TRIM39 and TRIM39 - TRIM26) in other mammals (mouse, rat, chimpanzee and human).

Contig 3 represented in this figure is a part within the MHC class I region as shown in the maps, Figures 5.1.1 and 5.4.1.

\subsubsection{Contig 4, the intervall TCTEX4 - MOG}

\subsubsection{Southern blot analysis of contig 4}

In Southern blot analysis, the clones $348 \mathrm{C} 21$ and $171 \mathrm{~K} 8$ presented four EcoRI fragments positive with Caja-G and HLA-B. Therefore, Callithrix also may have MHC class I gene(s) between TCTEX4 and MOG is well known for human and chimpanzee (Shiina et al., 2003). 


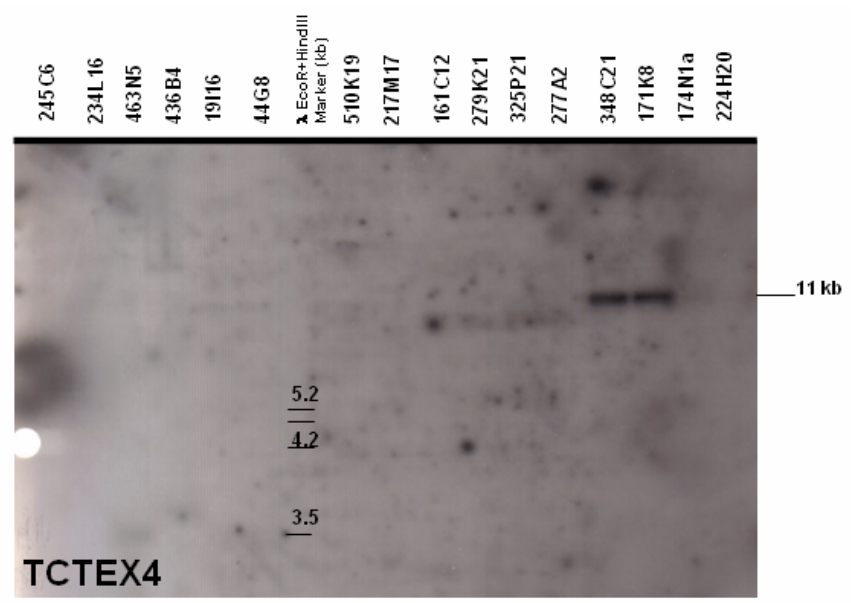

Figure 5.2.3.1.1: Autoradiograph after Southern blot hybridization of TCTEX4 framework gene probe with BAC-clone fragments (CHORI-259, Callithrix jacchus).

MHC-class I-positive BAC-clones were digested with restriction enzyme EcoRI. After gel electrophoresis and fingerprinting, their fragments were hybridized with said gene probe. DNA sample Lambda $(\lambda)$ EcoR/+HindIII used as ladder marker. kb values on the right margin calculated relative to marker DNA fragments which were seen by means of ethidium bromide fluorescence.

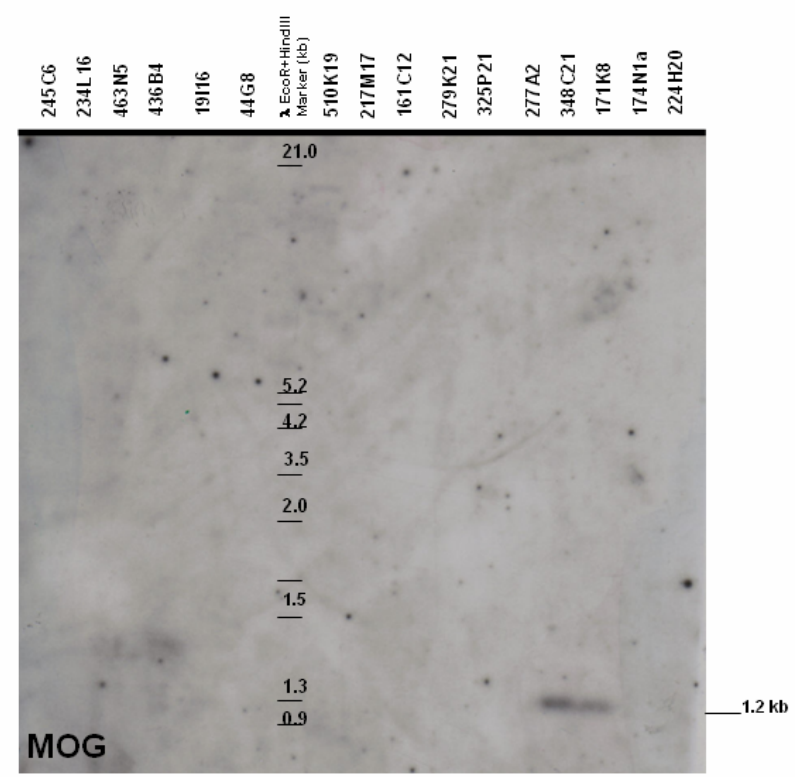

Figure 5.2.3.1.2: Autoradiograph after Southern blot hybridization of MOG framework gene probe with BAC-clone fragments (CHORI-259, Callithrix jacchus). 
MHC-class I positive BAC-clones were digested with restriction enzyme EcoRI. After gel electrophoresis and fingerprinting, their fragments were hybridized with said gene probe. DNA sample Lambda $(\lambda)$ EcoRI+HindIII used as ladder marker. kb values on the right margin calculated relative to marker DNA fragments which were seen by means of ethidium bromide fluorescence.

\subsubsection{Evaluation of hybridization results (contig 4)}

Here is shown an identical hybridization with of contig 4 and class I probes in these two clones suggesting them to contain the interval TCTEX4 - MOG of common marmoset.

- MOG: This framework gene probe detected one fragment of $1.2 \mathrm{~kb}$. These clones appeared to be overlapping: 348C21 and 171K8 (Figure 5.2.3.1.1).

- TCTEX4: This framework gene probe detected two fragments, both of $11 \mathrm{~kb}$. These clones appeared to be overlapping: 348C21 and 171K8 (Figure $5.2 .3 .1 .2)$.

- MHC class I-Caja-G: This framework gene probe detected two fragments, of $12 \mathrm{~kb}$ and $6 \mathrm{~kb}$. The same two clones matching also probes MOG and TCTEX4 appeared to be overlapping: 348C21 and 171 K8 (Figure 5.2.1.1.1).

- MHC class I HLA-B: This probe detected two fragments of $11 \mathrm{~kb}$ and $12 \mathrm{~kb}$ in clone $171 \mathrm{~K} 8$, and only one of $12 \mathrm{~kb}$ in clone 348C21 (Figure 5.2.1.1.12).

- MICA: This probe was negative for this contig (Figure 5.2.1.1.15).

\subsubsection{Contribution to a contig 4 map}

Contig 4 contains between the conserved frameworks genes TCTEX4 and MOG many MHC class I genes and pseudogenes intercalated with many other genes (see MHC class I maps to bp-scale, Figures 5.1.1 and 5.3.1.1). The interval has remarkably different lengths in the mammals investigated, approximately $50 \mathrm{~kb}$ in the rat RT1-complex (Hurt et al., 2004) $400 \mathrm{~kb}$ in contig 4 of Hs. This interval is conformed by two clones, however the sequence analysis strongly and extensively supports that this contig exist in Callithrix jacchus. 


\section{Contig 4}

\begin{tabular}{|c|c|c|c|c|c|c|}
\hline & \multicolumn{6}{|c|}{$\begin{array}{l}\text { HLA-B } \\
\text { ( Caja-G) }\end{array}$} \\
\hline & 7 & 6.5 & 7 & 11 & 12 & 1.2 \\
\hline$K 23: 348 \mathrm{C} 21$ & 7 & $?$ & $?$ & $?$ & 12 & 1.2 \\
\hline K24:171K8 & 7 & 6.5 & 7 & 11 & 12 & 1.2 \\
\hline
\end{tabular}

Figure 5.2.3.3.1: Two overlapping BAC clone inserts with framework genes of contig 4.

The order of the class I containing fragments $6.5,7$ and $11 \mathrm{~kb}$ remains an enigma. Nevertheless, it may be concluded, that these two clones must contain at least four HLA-B and Caja-G positive class I genes or pseudogenes, however, not necessarily between TCTEX4 and MOG.

Again, sequences established lateron, localized and as possible gene assigned in comparison to the Hs genome strongly suggest many homologoues Caja genes to exist in the interval of contig 4.

Complete evidence for class I gene maps based on gene assignment by comparison shall be coming from a complete sequencing that has been started already by Prof. T. Shiina using the clones selected by the screening described in chapter 5.1 of this thesis.

\subsection{PCR and sequence analysis of BAC clone inserts}

\subsubsection{Polymerase chain reaction (PCR) analysis}

Locus-specific PCR was used to characterize the clones by primer specific fragments which were sequenced partially or totally (see next chapter, 5.4). Oligonucleotide primers to POU5F1, MOG, K18, HLB 32, 29, 39, Caja-G and Caja-E were designed with primer-3 on the Web (http//www.genome.wi.mi.edu/cgi-bin/primer/primer3.cgi).

- For POU5F1 were found 7 positives clones (347K7, 347D1, 379F14, 459O20, 169G2, $123 \mathrm{H} 17$ and 99L19) containing a fragment of $450 \mathrm{bp}$ (see Figure 5.3.1).

- For K18T3 was found one clone (217M19) containing a fragment of $150 \mathrm{bp}$. 
- For Caja-G were found 5 positives clones (120D3, 271C5, 127E13, 44G8 and $171 \mathrm{~K} 8$ ) containing a fragment of $650 \mathrm{bp}$.

- For Caja-E were found eight positive clones (271C5, 18G14, 204C3, 127E13, 325P21, 277A2, 348C21 and 171K8) containing a fragment of $500 \mathrm{bp}$.

- For HLAB-32 were found 13 positive clones (120D3, 271C5, 127E13, 463N5, 325P21, 277A2, 224H20, 347K7, 347D1, 379F14, 459O20, $169 \mathrm{G} 2$ and $123 \mathrm{H} 17)$ containing a fragment 700 of bp.

- For HLAB-29 were found 12 positive clones (120D3, 271C5, 18G14, 261L9, 463N5, 161C12, 347K7, 347D1, 379F14, 459O20, 169G2 and 123H17) containing a fragment of $800 \mathrm{bp}$.

- For HLAB-39 were found three positive clones (261L9, 325P21, 277A2) with a fragment $850 \mathrm{bp}$.

- For Klexon 2 MHC consensus were found two positive clones (120D3 and 127E13) containing a fragment 700 of bp.

- $\quad$ MOG was negative in all clones (see Figure 5.3.1.1).

Acknowledgement: The primers HLA-B 29, 32 and 39 were kindly given to the author by Cornelia Rosner; the primer ALU was a gift of Martin Osther and a MICA DNA sample by Anne Averdam, all working in the DPZ, Göttingen.

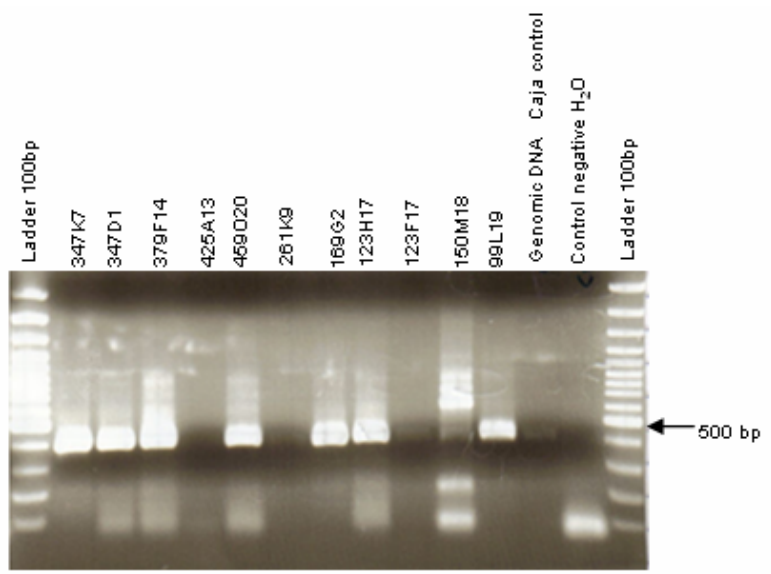

Figure 5.3.1.1 PCR with primer POU5F1.

Image of a $1 \%$ agarose gel stained with ethidium bromide $20 \mathrm{mg} / \mathrm{ml}$. Only clones positive with primer POU5F1 show fragments. The control with nuclear DNA from Callithrix jacchus (sample on the right, next to ladder). 


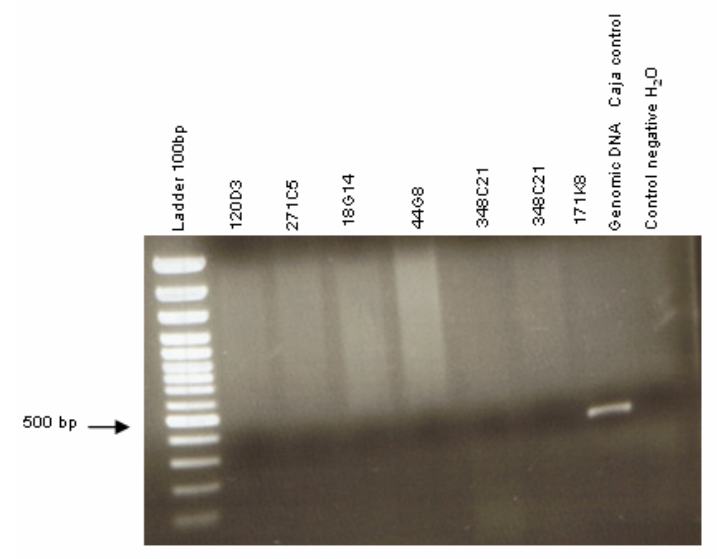

Figure 5.3.1.2 PCR with primer MOG.

Fluorescence photograph of a $1 \%$ agarose gel stained by ethidium bromide 20 $\mathrm{mg} / \mathrm{ml}$. Only the nuclear DNA from Callithrix jacchus was positive with MOG. 
Table 5.3.1.1: Genetic typification of BAC clone inserts by PCR. The table contains all clones tested with different primers from framework, Caja-G and HLA genes.

\begin{tabular}{|c|c|c|c|c|c|c|c|c|c|c|c|c|}
\hline Clone & $\begin{array}{l}\text { Caja G } \\
650 \text { bp }\end{array}$ & $\begin{array}{l}\text { Caja-E } \\
500 \text { bp }\end{array}$ & $\begin{array}{l}\text { Klexon2 } \\
\text { consenso }\end{array}$ & $\begin{array}{l}\text { TRIM39 } \\
1500 \text { bp }\end{array}$ & $\begin{array}{l}\text { TCF19 } \\
350 \text { bp }\end{array}$ & $\begin{array}{l}\text { TRIM10 } \\
400 \mathrm{bp} \\
900 \mathrm{bp} \\
\end{array}$ & $\begin{array}{l}\text { MOG } \\
350 \mathrm{bp}\end{array}$ & $\begin{array}{l}\text { HLA-B-32 } \\
700 \text { bp }\end{array}$ & $\begin{array}{l}\text { HLA-B-29 } \\
800 \text { bp }\end{array}$ & $\begin{array}{l}\text { HLA-B-39 } \\
850 \text { bp }\end{array}$ & $\begin{array}{l}\text { POU5F1 } \\
450 \mathrm{bp}\end{array}$ & $\begin{array}{l}\text { K18T3 } \\
150 \mathrm{bp}\end{array}$ \\
\hline $120 \mathrm{E} 3$ & + & - & + & - & - & + & - & + & + & - & - & - \\
\hline $271 \mathrm{C5}$ & + & + & - & - & - & - & - & + & + & - & - & - \\
\hline 18G14 & - & + & - & - & + & - & - & - & + & - & - & - \\
\hline 282L12 & - & - & - & - & + & - & - & - & - & - & - & - \\
\hline $204 C 3$ & - & + & - & - & - & - & - & - & - & - & - & - \\
\hline 127E13 & + & + & + & - & - & + & - & + & & - & - & - \\
\hline $239 N 18$ & - & - & - & - & - & - & - & - & - & - & - & - \\
\hline 193P12 & - & - & - & - & - & + & - & - & - & - & - & - \\
\hline 261L9 & - & - & - & - & - & + & - & - & + & + & - & - \\
\hline 282015 & - & - & + & - & - & - & - & - & - & - & - & - \\
\hline $245 \mathrm{C} 6$ & - & - & + & - & - & + & - & - & - & - & - & - \\
\hline 234L16 & - & - & + & - & - & + & - & - & - & - & - & - \\
\hline $463 N 5$ & - & - & + & - & - & + & - & + & + & - & - & - \\
\hline 436B4 & - & - & + & - & - & + & - & - & - & - & - & - \\
\hline $19 \mid 16$ & - & - & + & - & - & + & - & - & - & - & - & - \\
\hline $44 \mathrm{G} 8$ & + & - & + & + & - & - & - & - & - & - & - & - \\
\hline 510K19 & - & - & - & - & - & - & - & - & - & - & - & - \\
\hline $217 \mathrm{M} 17$ & - & - & - & - & - & + & - & - & - & - & - & + \\
\hline $161 \mathrm{C} 12$ & - & - & - & - & - & - & - & - & + & - & - & - \\
\hline $279 K 21$ & - & - & - & + & - & + & - & - & - & - & - & - \\
\hline $325 \mathrm{P} 21$ & - & + & - & + & - & - & - & + & - & + & - & - \\
\hline 277A2 & - & + & - & - & - & - & - & + & - & + & - & - \\
\hline $348 C 21$ & - & + & - & - & - & + & - & - & - & - & - & - \\
\hline $171 \mathrm{~K} 8$ & - & + & - & - & - & - & - & - & - & - & - & - \\
\hline $174 \mathrm{~N} 1$ & - & - & - & - & - & - & - & - & - & - & - & - \\
\hline $224 \mathrm{H} 20$ & - & - & - & + & - & + & - & + & - & - & - & - \\
\hline $347 \mathrm{~K} 7$ & - & - & - & + & + & - & - & + & + & - & + & - \\
\hline 347D1 & - & - & - & - & + & - & - & + & + & - & + & - \\
\hline $379 F 14$ & - & - & - & - & + & - & - & + & + & - & + & - \\
\hline $425 \mathrm{~A} 13$ & - & - & - & - & - & - & - & - & - & - & + & - \\
\hline 459020 & - & - & - & + & + & - & - & + & + & - & + & - \\
\hline $169 \mathrm{G} 2$ & - & - & - & - & - & - & - & + & + & - & + & - \\
\hline $123 \mathrm{H} 17$ & - & - & - & + & + & - & - & + & + & - & + & - \\
\hline 99L19 & - & - & - & - & + & - & - & - & - & - & + & - \\
\hline $334 \mathrm{C} 4$ & - & - & - & + & - & - & - & - & - & - & - & - \\
\hline 329M5 & - & - & - & - & - & - & - & - & - & - & - & - \\
\hline 379M5 & - & - & - & - & - & - & - & - & - & - & - & - \\
\hline 174N1 & - & - & - & - & - & - & - & - & - & - & - & - \\
\hline $485 C 4$ & - & - & - & - & - & - & - & - & - & - & - & - \\
\hline $\begin{array}{l}\text { Caja } \\
\text { DNAg }\end{array}$ & + & + & + & + & + & + & + & + & + & + & + & + \\
\hline
\end{tabular}




\subsubsection{Approximative alignment of sequences to homologoues DNA of Hs and other mammals by BLAST}

In an attempt to facilitate insight into unknown basic functions to be expected in he highly conserved framework, by an example from the MHC even from years to many (Amadou et al., 1995) ways for the analysis of their structure, i.e. the DNA sequence, were investigated. The fragments from BAC clones obtained by inverse PCR were excised from the gel, and extracted by Nal. They were sequenced e.g. by means of the primers T7.29, ptarbac2, ptarbac3, ptarbac4, TCF19 and HLA-B29, respectively. Several clones could be sequenced partially. The clone $161 \mathrm{C} 12$ has been sequenced completely by Dr. Takashi Shiina at Tokyo University School of Medicine in Tokyo, Japan.

After automated sequencing of fragments recovered from electropherograms, sequencing results were inspected in order to identify errors and ambiguities. These corrected sequences were transferred to BIOEDIT for correction of mistakes, and furthermore to BLASTVECTOR for removal of vector sequences. Genebank database was then searched using the BLASTIN algorithm in order to identify significant nucleotide matches. A value for similarity or homology of Callithrix jacchus sample sequences with database sequences of several species was calculated referring to the BAC-banks of these species. Significant matches were arbitrarily defined, first as those which showed sequence identity (homology) greater than $80 \%$ over a segment of DNA of not less than $30 \mathrm{bp}$ in length, and which gave a BLASTIN high scoring point of 300 or above at a significant $p$ value. To perform sample sequencing, by the use of pairs of primers, sequencing in two directions of reading was performed. 
Table 5.3.2.1: Gene assignment of MHC class I sequences by comparison with Hs and other mammals using BLAST giving \% identity for a best aligning part of a sequence.

$\psi$ designates pseudogenes otherwise abbreviated ps.

\begin{tabular}{|c|c|c|c|c|}
\hline Clone & Contig & Primer & $\begin{array}{l}\text { Sequence } \\
\text { (bp) }\end{array}$ & Species: in or near gene; homology \% \\
\hline \multirow[t]{2}{*}{$204 C 3$} & 1 & ptarbac3 & 438 & $\begin{array}{l}\text { Hs: pseudogene } 88 \% \\
\text { Mamu: MIC2 } 89 \%\end{array}$ \\
\hline & & T7.29 & 619 & $\begin{array}{l}\text { Hs: BAT2; NCR3 } 87 \% \\
\text { Mamu: BAT2; NCR3 } 88 \% \%\end{array}$ \\
\hline 510K19 & 1 & ptarbac3 & 211 & $\begin{array}{l}\text { Patr:Patr-C; } 88 \% \\
\text { Hs: } \psi \text { before OTF3; } 87 \% \\
\text { Mamu: Mamu-B18; } 86 \%\end{array}$ \\
\hline \multirow[t]{2}{*}{ 217M17 } & 1 & ptarbac3 & 208 & $\begin{array}{l}\text { Hs: MICA; } 88 \% \\
\text { Patr: MIC; } 87 \% \\
\text { Mamu: MIC; } 89 \%\end{array}$ \\
\hline & & T7.29 & 383 & $\begin{array}{l}\text { Hs: } \psi \text { HCGIIX4; } 90 \% \\
\text { Patr: MICA-B; } 90 \% \\
\text { Mamu: MIC3; } 90 \%\end{array}$ \\
\hline \multirow[t]{2}{*}{$347 K 7$} & 1 & ptarbac3 & 515 & $\begin{array}{l}\text { Hs: SEEK1 exon 1; } 84 \% \\
\text { Patr: SEEK1 exon } 1 ; 85 \% \\
\text { Mamu: C6orf15; } 85 \%\end{array}$ \\
\hline & & ptarbac4 & 363 & $\begin{array}{l}\text { Hs: HCGIX4; } 90 \% \\
\text { Patr: MICB; } 90 \% \\
\text { Mamu: MIC 3; } 90 \%\end{array}$ \\
\hline \multirow[t]{3}{*}{ 347D1 } & 1 & ptarbac3 & 518 & $\begin{array}{l}\text { Hs: SEEK1 exon } 1 ; 86 \% \\
\text { Patr: SEEK1 exon } 1 ; 86 \% \\
\text { Mamu: C6orf15 } 87 \%\end{array}$ \\
\hline & & ptarbac4 & 534 & $\begin{array}{l}\text { Hs: SEEK1 exon 1; } 84 \% \\
\text { Patr: SEEK1; } 84 \% \\
\text { Mamu: C6orf15; } 85 \%\end{array}$ \\
\hline & & TCF19 & 2314 & $\begin{array}{l}\text { Hs: TCF19 exon 2; } 90 \% \\
\text { Patr: TCF19; } 90 \% \\
\text { Mamu: TCF19; } 90 \% \\
\text { Rano: TCF19; } 89 \%\end{array}$ \\
\hline \multirow[t]{4}{*}{459020} & 1 & ptarbac3 & 624 & $\begin{array}{l}\text { Hs: } 3.8-1-4 ; 85 \% \\
\text { Patr: } \psi 3.8-1-4 ; 85 \% \\
\text { Mamu: } 3.8-1-4 ; 88 \%\end{array}$ \\
\hline & & ptarbac4 & 511 & $\begin{array}{l}\text { Hs: } 3.8-1-4 ; 87 \% \\
\text { Patr: } \psi 3.8-1-4 ; 86 \% \\
\text { Mamu: } \psi 3.8-1-4 ; 88 \%\end{array}$ \\
\hline & & T7.29 & 121 & $\begin{array}{l}\text { Hs: SEEK1; } 89 \% \\
\text { Patr: SEEK } 1 ; 89 \% \\
\text { Mamu: SEEK } 1 ; 88 \%\end{array}$ \\
\hline & & TCF19 & 258 & $\begin{array}{l}\text { Patr: TCF19 exon 2; } 92 \% \\
\text { Mamu: TCF19; } 91 \% \\
\text { Rano: TCF19; } 90 \% \\
\end{array}$ \\
\hline \multirow[t]{3}{*}{ 169G2 } & 1 & T7.29 & 207 & $\begin{array}{l}\text { Hs: HLA-B; } 94 \% \\
\text { Patr: Patr-B exon 8; } 95 \% \\
\text { Mamu: Mamu-B13; } 94 \%\end{array}$ \\
\hline & & ptarbac3 & 361 & $\begin{array}{l}\text { Hs: class I; } 95 \% \\
\text { Patr: class I; } 95 \% \\
\text { Mamu: class I; } 94 \%\end{array}$ \\
\hline & & ptarbac4 & 328 & $\begin{array}{l}\text { Hs: TCF19; } 89 \% \\
\text { Hs: SEEK } 1 ; 95 \% \\
\text { Patr: SEEK } 1 ; 95 \%\end{array}$ \\
\hline \multirow[t]{2}{*}{$123 \mathrm{H} 7$} & 1 & T7.29 & 527 & $\begin{array}{l}\text { Hs: TCF19; } 89 \% \\
\text { Patr: TCF19; } 89 \% \\
\text { Mamu: TCF19; } 89 \%\end{array}$ \\
\hline & & TCF19 & 567 & $\begin{array}{l}\text { Hs: TCF19; } 89 \% \\
\text { Mamu: TCF } 19 ; 89 \% \\
\text { Patr: TCF19; } 89 \%\end{array}$ \\
\hline 99L19 & 1 & TCF19 & 377 & $\begin{array}{l}\text { Hs: TCF } 19 \text { exon } 2 ; 89 \% \\
\text { Patr: TCF19 exon } 1 \text { and } 2 ; 92 \% \\
\text { Mamu: TCF } 19 ; 89 \% \\
\text { Rano: TCF } 19 ; 89 \%\end{array}$ \\
\hline $161 \mathrm{C} 12$ & 1 & $\begin{array}{l}\text { Unknown } \\
\text { primer of } \\
\text { Dr. Shiina }\end{array}$ & $3000^{*}$ & $\begin{array}{l}\text { Hs: BAT1 exon } 5 ; 90 \% \\
\text { Patr: BAT1 exon } 4 ; 88 \% \\
\text { Mamu: BAT1; } 88 \% \\
\text { Susc: BAT1 exon } 4 ; 90 \%\end{array}$ \\
\hline
\end{tabular}




\begin{tabular}{|c|c|c|c|c|}
\hline & & & & $\begin{array}{l}\text { Bota: BAT1; }<70 \% \\
\text { Mumu: BAT1a; }<70 \% \\
\text { Rano: BAT1a; }<70 \% \\
\text { Aime: BAT1a; }<70 \%\end{array}$ \\
\hline & & $\begin{array}{l}\text { Unknown } \\
\text { primer of } \\
\text { Dr. Shiina }\end{array}$ & $2994^{*}$ & $\begin{array}{l}\text { Patr: pseudogene HCGIV-2; } 88 \% \\
\text { Atbe: Atbe-Be3; } 91 \%\end{array}$ \\
\hline 261L9 & 1 & HLA-B 29 & 207 & $\begin{array}{l}\text { Hs: HLA-B; } 73 \% \\
\text { Patr: Patr-B; } 74 \%\end{array}$ \\
\hline Clone & Contig & Primer & bp & Species: in or near gene; homology \% \\
\hline $245 C 6$ & 3 & T7.29 & 280 & $\begin{array}{l}\text { Hs: TRIM15 exon 2; } 92 \% \\
\text { Patr: TRIM15 exon } 5 ; 94 \% \\
\text { Mamu: TRIM15; } 93 \%\end{array}$ \\
\hline \multirow[t]{2}{*}{ 463N5 } & 2 or 3 & HLA-B & 358 & $\begin{array}{l}\text { Hs: HLA-B; } 91 \% \\
\text { Patr: Patr-B; } 92 \% \\
\text { Mamu: Mamu-B13; } 88 \%\end{array}$ \\
\hline & & ptarbac3 & 365 & $\begin{array}{l}\text { Hs: HLA-E; } 86 \% \\
\text { Patr: Patr E; } 86 \% \\
\text { Mamu: Mamu-E; } 85 \%\end{array}$ \\
\hline 436B4 & 2 or 3 & $\mathrm{~T} 7.29$ & 247 & $\begin{array}{l}\text { Hs: HLA-B; } 94 \% \\
\text { Hs: HLA-B27; } 94 \% \\
\text { Patr: Patr-B; } 95 \% \\
\text { Mamu: Mamu-B10; } 96 \% \\
\end{array}$ \\
\hline \multirow[t]{2}{*}{ 325P21 } & 2 or 3 & $\mathrm{~T} 7.29$ & 323 & $\begin{array}{l}\text { Hs: TRIM10 exon 6; } 91 \% \\
\text { Mamu: TRIM10; } 88 \% \\
\text { Susc: TRIM10; } 92 \%\end{array}$ \\
\hline & & ptarbac3 & 171 & $\begin{array}{l}\text { Hs: HLA-G exon 3; } 92 \% \\
\text { Patr: Patr-G; } 94 \%\end{array}$ \\
\hline 279K21 & 2 or 3 & $\mathrm{~T} 7.29$ & 482 & Caja: Caja-E; $97 \%$ \\
\hline $224 \mathrm{H} 20$ & 3 & T7.29 & 159 & $\begin{array}{l}\text { Hs: TRIM10 exon 7; } 93 \% \\
\text { Patr: TRIM10-TRIM15;94 \% } \\
\text { Mamu: TRIM10; } 93 \%\end{array}$ \\
\hline Clone & Contig & Primer & bp & Species: in or near gene; homology \% \\
\hline $348 C 21$ & 4 & T7.29 & 485 & $\begin{array}{l}\text { Hs: MOG Intron 3; } 90 \% \\
\text { Susc: MOG; } 93 \%\end{array}$ \\
\hline \multirow[t]{3}{*}{$171 K 8$} & 4 & T7.29 & 637 & Hs: MOG; $88 \%$ \\
\hline & & ptarbac2 & 567 & Hs: MOG; $88 \%$ \\
\hline & & Caja-G & 362 & $\begin{array}{l}\text { Hs: HLA-C exons 2-3; } 90 \% \\
\text { Hs: HLA-C exons 2-3; } 89 \% \\
\text { Hs: HLA-C exons } 1-8 ; 89 \% \\
\text { Hs: HLA-C; } 89 \% \\
\text { Saoe: Saoe-G G*12; } 91 \% \\
\end{array}$ \\
\hline \multirow[t]{7}{*}{ 120D3 } & $\begin{array}{c}\text { Outside } \\
\text { contigs } \\
1-4\end{array}$ & HLA-B 32 b & 235 & $\begin{array}{l}\text { Hs: HLA-B; } 89 \% \\
\text { Hs: HLA-BC; } 89 \%\end{array}$ \\
\hline & & HLA-B 29 b & 235 & $\begin{array}{l}\text { Hs: HLA-B27; } 93 \% \\
\text { Hs: HLA-B; } 92 \%\end{array}$ \\
\hline & & HLA-B 32a & 431 & $\begin{array}{l}\text { Hs: HLA-Bw57; } 89 \% \\
\text { Hs: HLA-C; } 69 \%\end{array}$ \\
\hline & & $\begin{array}{l}\text { Caja-G } \\
2273\end{array}$ & 666 & $\begin{array}{l}\text { Hs: HLA-C; } 85 \% \\
\text { Hs: HLA-C exons } 1,2 \text { and } 3 ; 85 \% \\
\text { Saoe: } \psi \text { Saoe-G*19; } 93 \% \text {. } \\
\text { Saoe: } \psi \text { Saoe-G*12; } 92 \%\end{array}$ \\
\hline & & Caja-E & 363 & $\begin{array}{l}\text { Hs: HLA-C; } 88 \% \\
\text { Hs: HLA-Cw- } 0202 \text { allele, intron 2;88 \% } \\
\text { Saoe: } \psi \text { Saoe-G*19; } 95 \%\end{array}$ \\
\hline & & $\mathrm{MIC}$ & 142 & $\begin{array}{l}\text { Hs: -DR class I } \psi ; 87 \% \\
\text { Hs: HLA-B } 48 \text { exons } 1 ; 86 \% \\
\text { Hs: HLA-B gene exon 4; } 86 \% \\
\text { Sasc:Sasc-G; } 86 \%\end{array}$ \\
\hline & & Klex2 & 84 & $\begin{array}{l}\text { Saoe: } \psi \text { Saoe-G*20; } 93 \% \\
\text { Saoe: } \psi \text { Saoe-G*19; } 91 \% \\
\text { Saoe: } \psi \text { Saoe-G*12; } 91 \% \\
\text { Caja: Caja-G*03; } 93 \% \\
\text { Caja: Caja-G*02; } 93 \%\end{array}$ \\
\hline \multirow[t]{2}{*}{ 127E13 } & & HLA-B 29 a & 280 & $\begin{array}{l}\text { Hs: HLA-B27; } 93 \% \\
\text { Hs: HLA-B; } 92 \% \\
\text { Hs: HLA-GHKAJ; } 93 \%\end{array}$ \\
\hline & & HLA-B 29 b & 175 & $\begin{array}{l}\text { Hs: HLA-B27; } 89 \% \\
\text { Hs: HLA-B27; } 89 \%\end{array}$ \\
\hline \multirow[t]{2}{*}{ 193P12 } & & $\mathrm{T} 7.29$ & 693 & Hs: clone RP11-512E16; $86 \%$ \\
\hline & & T7.29 & 511 & Hs: clone RP11-512E16; $86 \%$ \\
\hline 282L12 & & HLA-B 29 & 272 & $\begin{array}{l}\text { Hs: MICA; } 89 \% \\
\text { Patr: TNF } \propto 88 \% \\
\text { Mamu: MIC2; } 89 \%\end{array}$ \\
\hline
\end{tabular}




\begin{tabular}{|l|c|c|l|}
\hline 18G14 & ptarbac3 & 279 & Vector ptarbac 2.1; 96 \% \\
\hline 19116 & T7.29 & 229 & Hs: RP 11-765; 96\% \\
\hline 174N2 & $\mathrm{T7.29}$ & 202 & Hs: CCNE1; 86 \% \\
\hline 271C5 & $\mathrm{T7.29}$ & 170 & Vector ptarbac 2.1; 92 \% \\
\hline & $\mathrm{T7.29}$ & 232 & Mamu:Mamu-L; 91 \% \\
\hline & HLAB-29 & 575 & $\begin{array}{l}\text { Hs: HLA-B27; 92 \% } \\
\text { Patr: Patr-B exon 8; 98 \% } \\
\text { Mamu: Mamu-B1; 94 \% }\end{array}$ \\
\hline
\end{tabular}

\subsubsection{Sequences of contig 1}

In the MHC class I contig number 1, 21 insert sequences were obtained from 11 BAC clones. These may be summarized as follows: 19 sequences were achieved partially, i.e. 14 by the primers T7.21, ptarbac3, ptarbac2 and ptarbac4, belonging to the ptarbac 2.1 vector, four sequences by a TCF19 specific primer and one sequence by the primer HLA-B 29. Two sequences of clone $161 \mathrm{C} 12$ could be established (Table 5.3.3.2). All DNA sequences established (55) are presented in the annex. In this chapter they are briefly described. For an approximate localization they have been first aligned by BLAST program reporting a localization at or near a gene and the percentage identity found (for a stretch of best alignment - may be only some 50 150 bp). These data have been colleted in table 5.3.3.2.

Table 5.3.3.1: References to species compared

\begin{tabular}{|c|c|c|}
\hline Species & Abbreviation & References \\
\hline Homo sapiens & $\mathrm{Hs}$ & $\begin{array}{l}\text { Shiina et al., } 2001 \\
\text { Iris et al., } 1993 \\
\text { Hirakawa et al., } 2001 \\
\text { Beck et al., } 2000 \\
\text { Rhode et al., } 1999 \\
\text { Mizuki et al., } 1997 \\
\text { Robinson et al., } 2000 \\
\text { Roth et al., 1995; } \\
\text { Gonzales et al., } 1996 .\end{array}$ \\
\hline Pan troglodites & Patr & Anzai et al., 2003. \\
\hline Maccaca mulata & Mamu & $\begin{array}{l}\text { Kulski et al., 2004; } \\
\text { Fukami-Kobayashi et al., } 2005\end{array}$ \\
\hline Mus musculus & Mumu & Strausberg et al., 2002 \\
\hline Rattus norvegicus & Rano & $\begin{array}{l}\text { Hurt et al., } 2004 \\
\text { Ku et al., } 1991 \\
\text { Reymond et al., } 2001 \\
\end{array}$ \\
\hline Ateles belzebuth & Atbe & $\begin{array}{l}\text { Sawai et al., } 2004 \\
\text { Daza-Vamenta et al., unpublished }\end{array}$ \\
\hline Danio reiro & Dare & Strausberg et al., 2002 \\
\hline Sus scrofa & Susc & $\begin{array}{l}\text { Peelman et al., } 1995 \\
\text { Ando et al., } 2005\end{array}$ \\
\hline Ailuropoda melanoleuca & Aime & Liu unpublished \\
\hline Bos tauros & Bota & Strausberg et al., 2002 \\
\hline
\end{tabular}




\section{Table 5.3.3.2: Sequenced clones from contig 1}

\begin{tabular}{|l|c|c|c|c|c|c|}
\hline \multicolumn{1}{|c|}{ Primers } & \multicolumn{7}{c|}{ Clones } \\
\hline parbac3 & $347 \mathrm{~K} 7$ & $459 \mathrm{O} 20$ & $217 \mathrm{M} 17$ & 347D1 & $204 \mathrm{C} 3$ & $510 \mathrm{~K} 19$ \\
\hline ptarbac4 & $347 \mathrm{~K} 7$ & $459 \mathrm{O} 20$ & - & $347 \mathrm{D} 1$ & - & $169 \mathrm{G} 2$ \\
\hline T7.29 & $123 \mathrm{H} 7$ & $459 \mathrm{O} 20$ & $217 \mathrm{M} 17$ & - & $204 \mathrm{C} 3$ & $261 \mathrm{~L} 9$ \\
\hline TCF19 & $123 \mathrm{H} 7$ & $459 \mathrm{O} 20$ & - & $347 \mathrm{D} 1$ & $99 \mathrm{~L} 19$ & - \\
\hline HLA-B29 & - & - & - & - & - & $261 \mathrm{~L} 9$ \\
\hline Dr. Shiina & - & - & - & - & - & $161 \mathrm{C} 12$ \\
\hline
\end{tabular}

\subsubsection{Clone 204C3}

One partial insert sequence (1) of clone 204C3 obtained has a length of $438 \mathrm{bp}$. It was sequenced using primer ptarbac3. With the vector sequence tested it contains no significant similarities. According to Genebank database search, the sequence aligns in Homo sapiens (Hs), Macaca mulatta (Mamu) and Pan troglodytes (Patr) near the MIC gene. The respective homologies are $88 \%$ in $\mathrm{Hs}, 89 \%$ in Mamu, and $87 \%$ in Patr.

A second sequence (2) obtained by primer T7.29 has a length of $619 \mathrm{bp}$. It contains no significant similarities with the vector sequence. It aligns in Hs near the BAT2 region at a homology of $87 \%$, in Mamu, however, near the Natural Cytotoxicity Triggering Receptor 3 (NCR3) at 88 \% homology.

\subsubsection{Clone 510K19}

One partial insert sequence (3) obtained of clone 510K19 has a length of $211 \mathrm{bp}$. It was sequenced by means of primer ptarbac3, the vector sequence has a strong match at one end, the positions 157 - 207, and Hs, Mamu and Patr the sequence aligns with regions near the MIC gene. The respective homologies are: $88 \%$ in $\mathrm{Hs}$, $87 \%$ in Patr, and. 89 \% in Mamu.

\subsubsection{Clone 217M17}

One partial insert sequence (4) of clone $217 \mathrm{M} 7$ obtained has a length of $208 \mathrm{bp}$. It was sequenced by means of primer ptarbac3. At one end, the positions 157 - 207 It has a strong match with the vector sequence. The sequence aligns in both $\mathrm{Hs}$ and Patr to regions near the HLA complex group 2 pseudogene (HCGII-2). The respective homologies are $86 \%$ in $\mathrm{Hs}$ and $88 \%$ in Patr.

A second sequence (5) obtained using primer T7.29 has a length of $383 \mathrm{bp}$. It contains no significant similarities with the vector sequence. It aligns in Hs near the 
HCGIX-4, in Patr near the MICA-B, and in Mamu near the MIC3, its homology appears $90 \%$ in Hs, 90\% in Patr and $90 \%$ in Mamu, respectively.

\subsubsection{Clone 347K7}

One partial insert sequence (6) clone $347 \mathrm{~K} 7$ obtained has a length of $515 \mathrm{bp}$. It was sequenced using primer ptarbac3. It has a strong match with the vector sequence at one end, the positions 464 - 515. This sequence aligns both in Hs and Patr near SEEK1 exon 1, in Mamu, however, near C6orf15. The respective homologies are 84 $\%$ in $\mathrm{Hs}, 85 \%$ in Patr and $85 \%$ in Mamu.

A second sequence (7) obtained by means of primer ptarbac4 has a length of 363 bp. It shows no significant similarities with the vector sequence. It aligns, in Hs near the HCGIX-4, in Patr near the MICA-B and in Mamu near the MIC3. Homologies are of $90 \%$ in $\mathrm{Hs}, 90 \%$ in Patr and $90 \%$ in Mamu.

\subsubsection{Clone 347D1}

One partial insert sequence (8) of clone 347D1 obtained has a length of $518 \mathrm{bp}$. It was sequenced by means of primer ptarbac3. This sequence has a weak match with the vector means (weak here 1 random match expected in 40 queries of a length of $350 \mathrm{~kb}$ ), namely, a terminal match with score 16 to 18 , an internal match with score 23 to 24 at one end, the positions 468 - 491. The sequence aligns in Hs near the SEEK1 exon 1, in Patr near the SEEK1 and in Mamu near the C6orf15. The respective homologies are $86 \%$ in $\mathrm{Hs}, 86 \%$ in Patr and $87 \%$ in Mamu.

A second sequence (9) obtained by primer ptarbac4 has a length of $534 \mathrm{bp}$. It has has a strong match one end, the vector sequence in its positions 505 - 531, with a weak match in its positions 7 - 30. The sequence aligns in Hs near the SEEK1 exon 1, in Patr near the SEEK1 exon 1 and in Mamu near the C6orf15. The respective homologies are $84 \%$ in Hs, $84 \%$ in Patr and $85 \%$ in Mamu.

A third sequence (10) obtained using primer TCF19 from humans has a length of $2314 \mathrm{bp}$. The sequence aligns in Hs to the TCF19 gene exon 2, and in Patr, Mamu and Rattus norvegicus (Rano) also somewhere to the TCF 19 gene. The respective homologies are of 90 \% in Hs, 90 \% in Patr, 90 \% in Mamu and 89 \% in Rano. 


\subsubsection{Clone 459020}

One partial insert sequence (11) of clone 459020 obtained has a length of $624 \mathrm{bp}$. It was sequenced by means of primer ptarbac3. The sequence aligns in Hs pseudogene 3.8-1.1, in Patr pseudogene 3.8-1.2 and in Mamu near the pseudogene 3.8-1-09. The respective homologies are $85 \%$ in $\mathrm{Hs} 88 \%, 85 \%$ in Patr and in Mamu.

A second sequence (12) obtained with primer ptarbac4 has a length of $511 \mathrm{bp}$. This sequence has moderate match the vector in positions $1-29$. The sequence aligns in Hs to pseudogene 3.8-1-04, in Patr to pseudogene 3.8-1-04 and in Mamu to pseudogene 3.8-1-04. The respective homologies are $87 \%$ in $\mathrm{Hs} 86 \%$, in Patr and $88 \%$ in Mamu.

A third sequence (15) obtained using primer TCF19 has a length of $258 \mathrm{bp}$. This sequence aligns to the TCF19 gene in Hs, in Mamu and in Rano; in Patr, however, to the TCF19 gene exon 2. The respective homologies are $92 \%$ in Hs, $92 \%$ in Patr, 91 $\%$ in Mamu and $90 \%$ in Rano.

\subsubsection{Clone 169G2}

The partial insert sequence (16) of clone 169G2 obtained has a length of $207 \mathrm{bp}$. It was sequenced using primer T7.29. It contains no significant similarities with the vector sequence. The sequence aligns in Hs at HLA-B, in Patr near the Patr-B exon 8 and in Mamu near the Mamu-B13. Its respective homologies are $94 \%$ in $\mathrm{Hs}, 95 \%$ in Patr and $94 \%$ in Mamu.

\subsubsection{Clone 123H7}

One partial insert sequence (19) of clone $123 \mathrm{H} 7$ obtained using primer $\mathrm{T} 7.29$ has a length of $527 \mathrm{bp}$. It contains no significant similarities with the vector sequence. The sequence aligns near the TCF19 gene in Hs and Patr, in Mamu, however, in the TCF19 gene*). Its respective homologies are $89 \%$ in $\mathrm{Hs}, 89 \%$ in Patr and $89 \%$ in Mamu. *) Such alledged differences should be checked by alignment via a program like Clustal. BLAST gives rather a first approximation.

A second sequence (20) obtained using primer TCF19 has a length of $567 \mathrm{bp}$. This sequence aligns to the TCF19 gene in Hs, Mamu and Rano, yet in Patr to the TCF19 gene exon 2. The respective homologies are $91 \%$ in $\mathrm{Hs}, 90 \%$ in Patr, $90 \%$ in Mamu and $86 \%$ in Rano. 


\subsubsection{Clone 99L19}

A partial insert sequence (21) obtained of 99L19 using primer TCF19 has a length of $377 \mathrm{bp}$. This sequence aligns to the TCF19 gene in Hs, Mamu, and Rano. It aligns in Patr to the TCF19 gene exon 1 and 2. The respective homologies are $89 \%$ in Hs, 92 \% in Patr, 89 \% in Mamu and $89 \%$ in Rano.

\subsubsection{Clone 161C12}

One sequence obtained of clone 161C12 has a length of $2994 \mathrm{bp}$. It contains no significant similarities with the vector sequence. The sequence aligns in Ateles belzebuth (Atbe) with Atbe-B2 and Atbe-B3, in Hs and Patr, however, near the HCG IV-2. The respective homologies are $88 \%$ in Hs and Patr, $91 \%$ in Atbe and $89 \%$ in Mamu.

A second sequence obtained has a length of $3000 \mathrm{bp}$. It contains no significant similarities with the vector sequence. The sequence aligns in Hs with the BAT1 exon 5; with the BAT1 exon 4 in Patr and in Sus scrofa (Sucr); with the BAT1 in Mamu, Ailuropoda melanoleuca (Aime) and Bos tauros (Bota); with the BAT1a in Rano, Danio reiro (Dare) and Mus Musculus (Mumu). The respective homologies are $90 \%$ in $\mathrm{Hs}, 90$ \% in Patr, 89 \% in Mamu, 90 \% in Sucr, in Bota, Mumu, Rano and Aime data for $\%$ are not available.

\subsubsection{Clone 261L9}

The partial insert sequence (22) of clone 261L9 obtained using primer HLA-B.29 has a length of $207 \mathrm{bp}$. It contains no significant similarities with the vector sequence. The sequence aligns in Hs near the HLA-B exon 7, in Patr near the TRIM39 gene and in Mamu the Mamu-B13 gene. The respective homologies are $94 \%$ in $\mathrm{Hs}, 95 \%$ in Patr and $94 \%$ in Mamu.

\subsubsection{Sequences of contig 3}

In the contigs 2 and 3, 8 partiality sequences were obtained. These were sequenced by means of primers belonging to the ptarbac 2.1 vector. In summary: five sequences of the clones 245C6, 463N5, 436B4, 325P21 and 224H20 were obtained by means of the primer T7.29 and 3 sequences of the clones 279K21, 463N5 and 325P21 were achieved using the primer ptarbac3. 


\section{Sequence analysis of clones belonging to contig 3}

The sequence analysis to clone is interest because indicates to find framework genes, to confirm the presence of MHC class I gene and HLA-B.

The clones 325P21 and $224 \mathrm{H} 20$ showed the presence of the framework gene TRIM10. The clone 245C6 presented the framework gene TRIM15.

The organization of this frameworks is very similar to that described for mouse, rat and humans (Fukami-Kobayashi et al., 2005; Hurt et al., 2004; Shiina et al., 2004; Kulski et al., 2004).

The clones 224H20, 463N5 and 436B4 showed the presence of the HLA-B gene that is consistent with the Southern blot analysis, the primer T7.29 indicates the position of this HLA-B genes are in direction of CAT56, however is not easy to place in the map.

The clone $224 \mathrm{H} 20$ showed the presence of HLA-G, this primer T7.29 indicates the position of this HLA-G gene is in direction TRIM39, that is interest to clarify because this gene is present in the interval TCTEX4-MOG in humans (Pham-Dinh et al., 1993).

\subsubsection{Clone 245C6}

The partial insert sequence (29) of clone $245 \mathrm{C} 6$ obtained has a length of $280 \mathrm{bp}$. It was sequenced by means of primer T7.29, and showed no significant similarities with the vector sequence. The sequence aligns with the TRIM15 exon 2 in Hs and in Patr; however with the TRIM15 gene in Mamu and in Rano. The respective homologies are 84 \% in Hs, 94 \% in Patr, 93 \% in Mamu and 86 \% in Rano.

\subsubsection{Clone 463N5}

One partial insert sequence (30) of clone $463 \mathrm{~N} 5$ obtained has a length of $358 \mathrm{bp}$. It was sequenced using primer T7.29, and contains no significant similarities with the vector sequence. The sequence aligns in Hs near the HLA-B exon 8, in Patr the PatrB exon 8 and in Mamu near the Mamu-B13. The respective homologies are $91 \%$ in Hs, $92 \%$ in Patr and $88 \%$ in Mamu.

A second sequence (31) obtained has a length of $365 \mathrm{bp}$. It was sequenced using primer ptarbac3, and showed no significant similarity with the vector sequence. The sequence aligns in Patr near the Patr-E exon1 and in Mamu near the Mamu-E gene. The respective homologies are $86 \%$ in Patr and $85 \%$ in Mamu. 


\subsubsection{Clone 436B4}

The partial insert sequence (32) of clone 436B4 obtained has a length of $247 \mathrm{bp}$. It was sequenced using primer T7.29, and showed no significant similarities with the vector sequence. The sequence aligns in Hs near the HLA-B, in Patr near the Patr-B exon 8 and in Mamu the pseudogene Mamu-B10. The respective homologies are 94 $\%$ in $\mathrm{Hs}, 95 \%$ in Patr and 96 \% in Mamu.

\subsubsection{Clone 325P21}

One partial insert sequence (33) of clone 325P21 obtained has a length of $323 \mathrm{bp}$. It was sequenced using primer T7.29, and showed no significant similarity with the vector sequence. This sequence aligns the TRIM10 exon 6 in $\mathrm{Hs}$, in Patr and in Mamu, likewise, the TRIM10 gene in Rano and in Mumu, near the TRIM10 gene in Sucr. The respective homologies are $91 \%$ in $\mathrm{Hs}, 89 \%$ in Patr, 88 \% in Mamu, 92 \% in Susc, $97 \%$ in Rano and $94 \%$ in Mumu.

A second sequence (34) obtained has a length of $171 \mathrm{bp}$. It was sequenced using primer ptarbac3. It contains no significant similarities with the vector sequence. Quite differently the sequence aligns in Hs near the HLA-G gene and in Patr near the Patr$\mathrm{G}$ exon 3 and in Mamu near the pseudogene MamuG-2. The respective homologies are $94 \%$ in Hs, 95 \% in Patr and 96 \% in Mamu.

\subsubsection{Clone 279K21}

The partial insert sequence (35) of clone $279 \mathrm{~K} 2$ obtained has a length of $482 \mathrm{bp}$. It was sequenced using primer T7.29, and showed no significant similarities with the vector sequence. The sequence aligns in Hs near the HCGII-4 and in Mamu near the pseudogene human LOC282911 (HCG11-4). The respective homologies are $98 \%$ in $\mathrm{Hs}$ and $83 \%$ in Mamu. The respective homologies are $94 \%$ in $\mathrm{Hs}$ and $96 \%$ in Mamu.

\subsubsection{Clone 224H2O}

The partial insert sequence (36) of clone $224 \mathrm{H} 20$ obtained has a length of $159 \mathrm{bp}$. It was sequenced using primer T7.29, and showed no significant similarities with the vector sequence. This sequence aligns in Hs near the TRIM10 exon 7 and in Mamu near the TRIM10 gene. The respective homologies are $93 \%$ in $\mathrm{Hs}$ and $93 \%$ in Mamu. 


\subsubsection{Sequences of contig 4}

\section{Sequence analysis of clones belonging to contig 4}

In this contig number 4, 3 partial sequences were obtained. Two of these replicated were by means of primers belonging to the ptarbac 2.1 vector: two different sequences (although of equal bp size) of the clones $348 \mathrm{C} 21$ and $171 \mathrm{~K} 8$, respectively. One further sequence was obtained of the clone $171 \mathrm{~K} 8$ with the primer ptarbac2.

\subsubsection{Clone $348 \mathrm{C} 21$}

The partial insert sequence (37) of clone $348 \mathrm{C} 21$ obtained has a length of $485 \mathrm{bp}$. It was sequenced using primer T7.29. It contains no significant similarities with the vector sequence. The sequence aligns in Hs near the MOG exon 3 and intron 3 , and in Sucr near the MOG. The respective homologies are $93 \%$ in $\mathrm{Hs}$ and $93 \%$ in Sucr.

\subsubsection{Clone 171K8}

One partial insert sequence (38) of clone $171 \mathrm{~K} 8$ obtained has a length of $637 \mathrm{bp}$. It was sequenced using primer T7.29, and showed no significant similarities with the vector sequence. The sequence aligns in Hs near the MOG gene and has a homology of 88 \% in Hs (Roth et al., 1995; Gonzales et al., 1996).

A second sequence (39) has a length of $567 \mathrm{bp}$. It was sequenced using primer ptarbac2. It showed a strong match at one end, the positions 5 - 39 with the vector sequence. This sequence aligns in Hs near MOG. The respective homology is $88 \%$ in Hs (Roth et al., 1995; Gonzales et al., 1996).

\subsection{Identification of the Callithrix jacchus sequences obtained by comparative alignment}

5.4.1 Programs used for identification of sequences by localization and gene assignment

First step in the search for alignment was searching for homology and percent identity of the sequences by means of the BLAST program. This step gave information on the sequence type (location at or near a gene or intergenic sequence), the species in which the homology was found, the percentage of a maximum identity and the genomic position in the human genome compared. BLAST algorithms 
program shows a specific part within a sequence having the best score (Altschul et al., 1997). BLAST, however, gives no information for parts of lower identity.

Second step was to take information for other species and the query sequence and to align both sequences in Clustal $X$. This step allowed complete alignment between gene sequences and comparative DNA. Some sequences needed a modified approach, in i.e. using the Gene runner program, enabling correct alignment with the reverse or complementary sequence.

The third step was to calculate manually comparative genomic bp positions in the human genome.

Six of the 55 sequences were formally translated into amino acid sequences by means of the Swiss-prot program: K19-161C12-TS1, K19-161C12-TS2, K28-347D1TCF19, K24-171K8ptarbac2-38, K24-171K8ptarbac2-39 and K7-239N18T729-41 were informative. These sequences were thereupon examined by the AUGUSTUS program for gene prediction, and by Clustal $\mathrm{X}$ for amino acid alignment.

Some of the problems remaining resolved or critical in sequence aligning and evaluation will be mentioned in the discussion.

Figure 5.4.1: Gene map of the Hs MHC class I region showing aligment of the BAC clone sequences as found by BLAST and / or Clustal X. bp-scale map of the Hs MHC class I region: 1st and last bp of genes and sequences calculated for entry into a bp scale of the Venter genome, chromosome 6. For a more convenient display of all known Hs genes of the region and comparative alignment of the 55 Callithrix jacchus BAC clone sequences, the scale has been locally expanded from 10 to 1 or $0.1 \mathrm{~kb}$ per graduation line, respectively. See next three pages. 
Figure 5.4.1.: bp-scale map of the Hs MHC class I region (legend on preceding page)

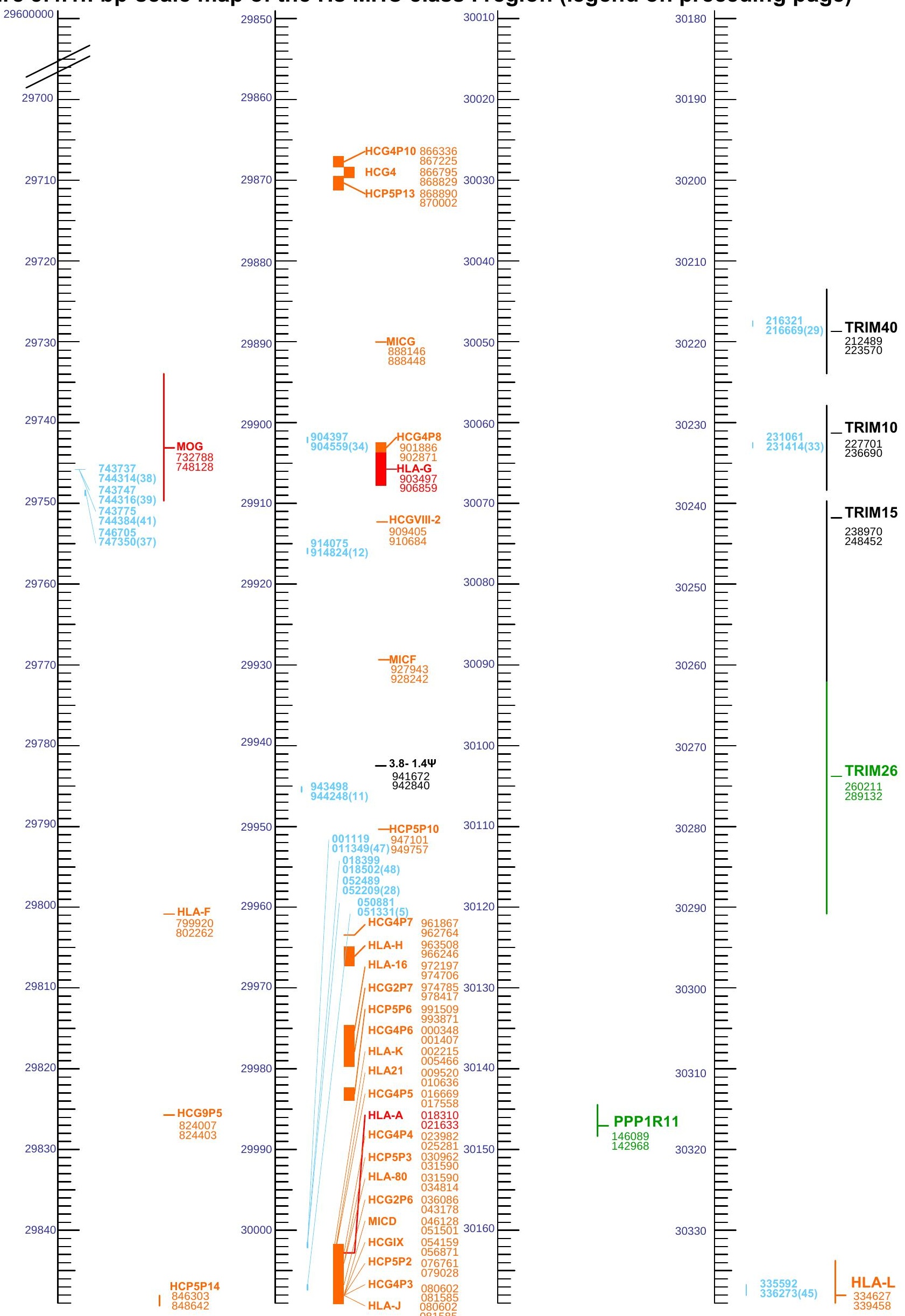



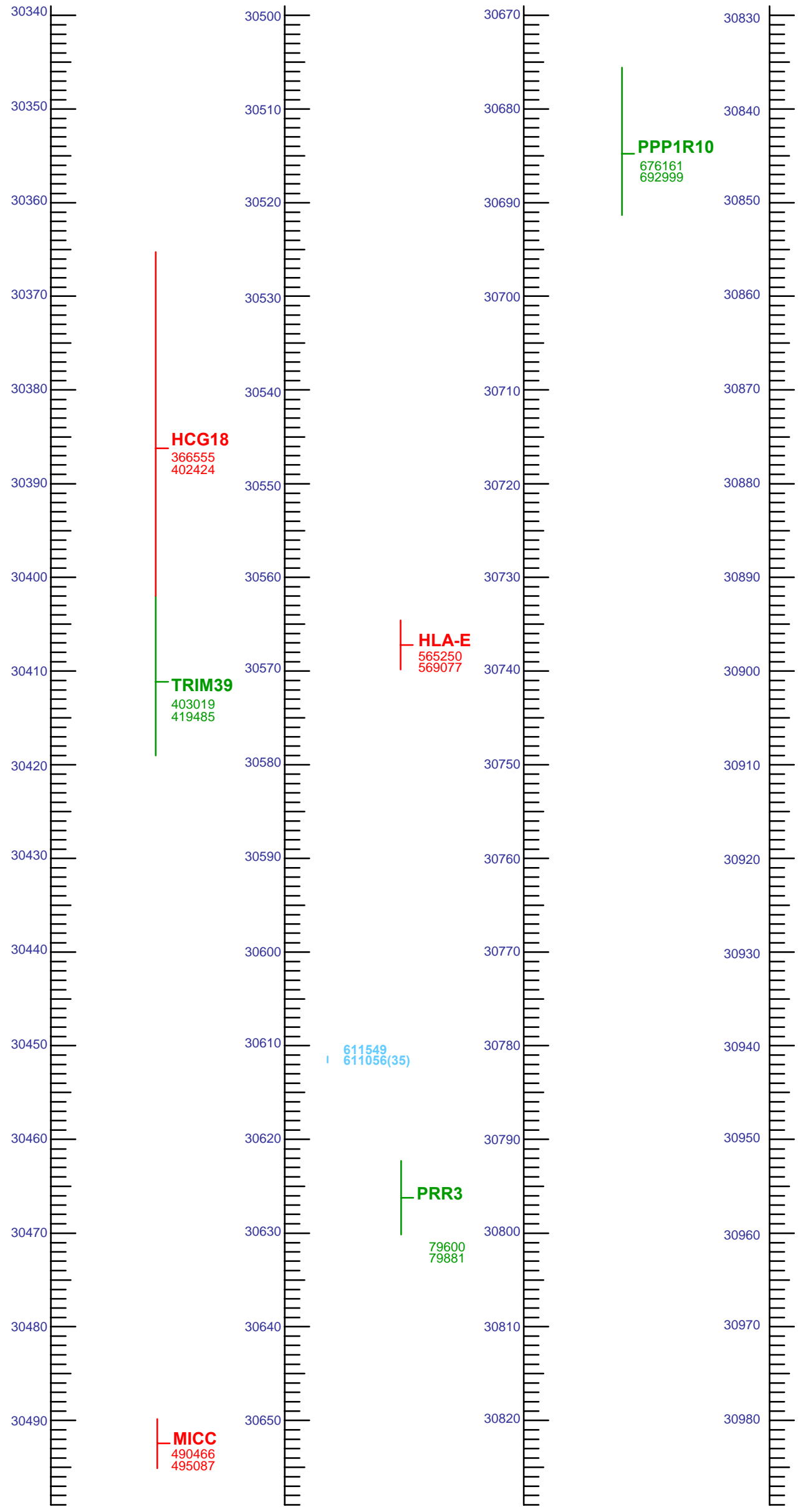


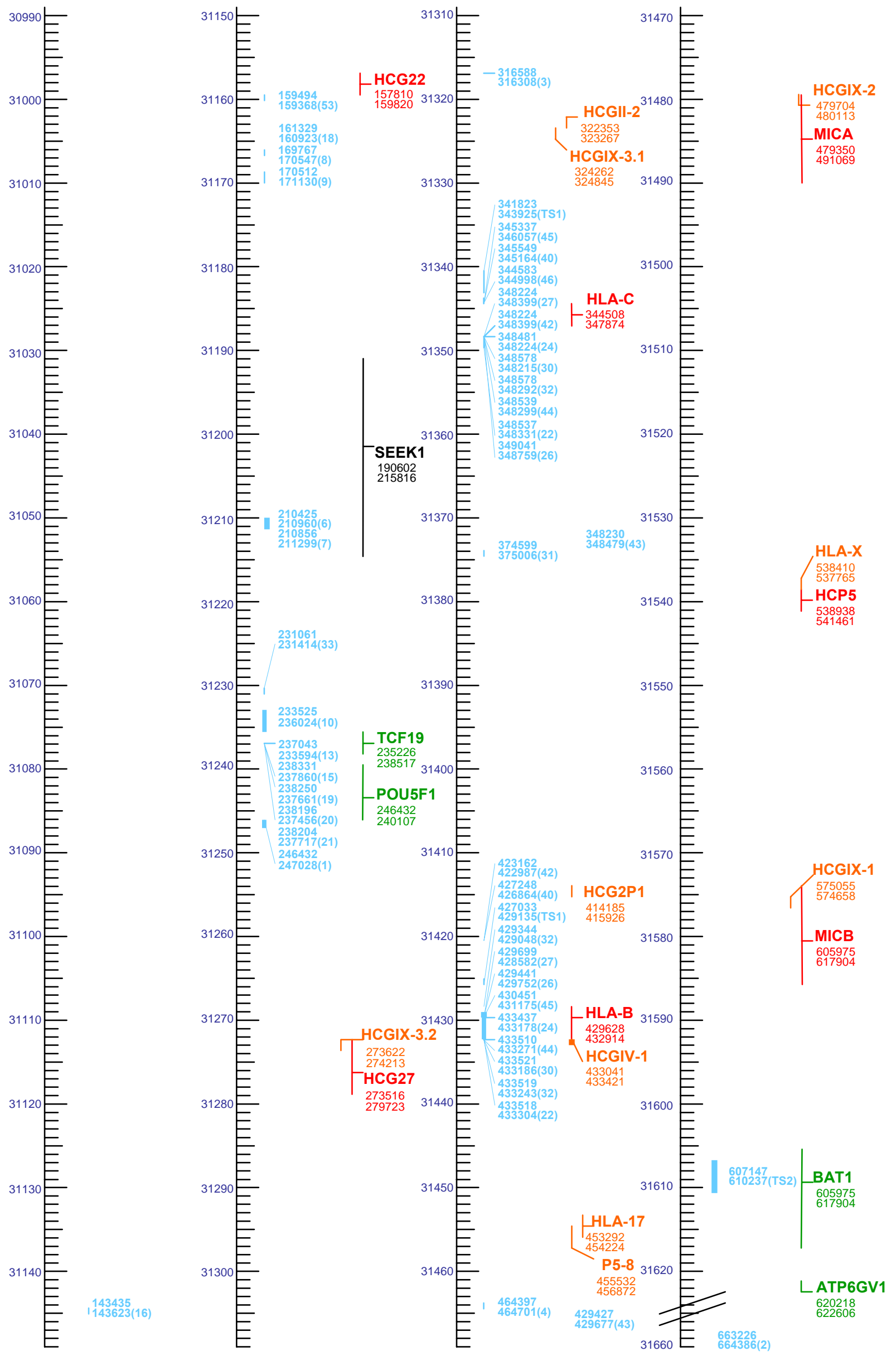


Table 5.4.1.1: Comparative definition of the first 55 MHC class I sequences of

Callithrix jacchus by BAC clone and primer used, length in bp obtained by PCR, first bp on the scale of Shiina, Venter genome numbers of sequence, \% identity of aligning part within or near a Hs gene.

\begin{tabular}{|c|c|c|c|c|c|c|}
\hline$\#$ & $\begin{array}{l}\text { Clone- } \\
\text { contig }\end{array}$ & Primer & $\begin{array}{l}\text { Sequ. } \\
\text { length } \\
\text { (bp) }\end{array}$ & $\begin{array}{l}\text { First bp } \\
\text { on } \\
\text { Shiina } \\
\text { scale }\end{array}$ & $\begin{array}{l}\text { First Venter genome bp number } \\
\text { of sequence } I 1^{\text {st }}-\text { last bp } \\
\text { aligned }: \% \text { identity with } \mathrm{Hs}^{2}\end{array}$ & $\begin{array}{l}\text { Gene hit or near }{ }^{3} \text { : defined by its } \\
\text { first - last Venter genome } \\
\text { numbers }\end{array}$ \\
\hline 1. & 204C3-1 & ptarbac3 & 438 & 744235 & 31247042 /247028 - 246432: 88 & HCGIX-3.2: 31247042 - 31274213 \\
\hline 2. & 204C3-1 & T729 & 619 & 349000 & 31662883 / 663226 - 664386: 87 & 1C7: $31664932-31323267$ \\
\hline 3. & $510 \mathrm{~K} 19-1$ & ptarbac3 & 211 & 696000 & 31315718 / $316308-316588: 88$ & HCGII-2: 31320349 - 31323267 \\
\hline 4. & 217M17-1 & ptarbac3 & 208 & 621000 & 31464017 / 464397 - 464701: 86 & HCGII-1: 31414185 - 31415110 \\
\hline 5. & 217M17-1 & T729 & 383 & 560000 & 30050871 / 050881 - 051331: 90 & HCG-IX: 30054159 - 30056871 \\
\hline 6. & $347 \mathrm{~K} 7-1$ & ptarbac & 491 & 847000 & $31210397 / 210425$ - 210960: 84 & SEEK1: 31190602 - 31215816 \\
\hline 7. & $347 \mathrm{~K} 7-1$ & ptarbac4 & 363 & 847000 & 31210397 / 210856 - 211299: 90 & SEEK1: 31190602 - 31215816 \\
\hline 8. & 347D1-1 & ptarbac3 & 518 & 845500 & 31169307 / 169767 - 170547: 86 & HCG22: 31157810 - 31159820 \\
\hline 9. & 347D1-1 & ptarbac4 & 534 & 847000 & 31170107 / 170512 - 171130: 84 & HCG22: $31157810-31159820$ \\
\hline 10. & $347 D 1-1$ & TCF19 & 2314 & & 31236549 / $236024-233525: 92$ & TCF19: $31235226-31238517$ \\
\hline 11. & $459020-1$ & ptarbac3 & 624 & 472000 & 29942715 / 943498 - 944248: 85 & $3.8-1.4: 29941672-29942840$ \\
\hline 12. & $459020-1$ & ptarbac4 & 511 & 166000 & 29913972 / 914075 - 914824: 86 & MICF: 29928242 - 29927943 \\
\hline 13. & $459020-1$ & T729 & 121 & & 31237214 / $237043-233594$ & TCF19: $31235226-31238517$ \\
\hline 14. & $459020-1$ & T729 & 159 & & No alignment in Hs localized & \\
\hline 15. & $459020-1$ & TCF19 & 258 & & 31235226 / 238331 - 237860: 92 & TCF19: $31235226-31238517$ \\
\hline 16. & $169 \mathrm{G} 2-1$ & T729 & 181 & & 31140485 / $143435-143623: 94$ & HCG22: $31157810-31159820$ \\
\hline 17. & $459020-1$ & ptarbac4 & 121 & & No alignment in Hs localized & \\
\hline 18. & 169G2-1 & ptarbac4 & 328 & 882000 & 31160924 / 160923 - 161329: 94 & HCG22: 31157810 - 31159820 \\
\hline 19. & $123 \mathrm{H} 7-1$ & T729 & 527 & & 31237984 / 237661 - 238250: 89 & TCF19: 31235226 - 31238517 \\
\hline 20. & 169G2-1 & TCF19 & 567 & & 31237876 / $237456-238196: 94$ & TCF19: $31235226-31238517$ \\
\hline 21. & 99L19-1 & TCF19 & 377 & & 31237479 / $237717-238204: 89$ & TCF19: $31235226-31238517$ \\
\hline 1TS & $161 C 12-1$ & $?$ & 2994 & $\begin{array}{l}583800 \\
668236 \\
\end{array}$ & 31432323 / 432291 - 429329: 88 & $\begin{array}{l}\text { HLA-B: } 31429628-31432914 \\
\text { HLA-C: } 31347834-31347834\end{array}$ \\
\hline 2TS & $161 \mathrm{C} 12-1$ & $?$ & 3000 & & 31607147 / $607147-610237: 90$ & BAT1: 31605975 - 31617904 \\
\hline 22. & 261L9-1 & HLA-B 29 & 207 & $\begin{array}{l}590669 \\
675217\end{array}$ & 31430382 / 430382 - 429958: 94 & $\begin{array}{l}\text { HLA-B: } 31429628-31432914 \\
\text { HLA-C: } 31347834-31347834\end{array}$ \\
\hline 23. & $271 \mathrm{C5}-1$ & $\mathrm{~T} 7.29$ & 170 & & ptarbac2.1 = vector & \\
\hline 24. & $271 C 5-1$ & HLA-B 32A & 575 & 590206 & 31429698 / 430105 - 429698: 95 & $\begin{array}{l}\text { HLA-B: } 31429628-31432914 \\
\text { HLA-C: } 31347834-31347834\end{array}$ \\
\hline 25. & $271 C 5-1$ & T7.29 & 170 & & ptarbac2.1 = vector & \\
\hline 26. & 127E13-1 & HLA-B29 & 280 & $\begin{array}{l}590910 \\
675173\end{array}$ & 31432636 / 432681 - 433030: 93 & $\begin{array}{l}\text { HLA-B: } 31429628-31432914 \\
\text { HLA-C: (after HLA-C) }\end{array}$ \\
\hline 27. & 127E13-1 & HLA-B29B & 175 & $\begin{array}{l}590070 \\
674636 \\
\end{array}$ & 31432914 / 432071 - 431868: 89 & $\begin{array}{l}\text { HLA-B: } 31429628-31432914 \\
\text { HLA-C: } 31347834-31347834\end{array}$ \\
\hline 28. & 282L12-1 & ptarbac3 & 272 & & 30052489 / $052489-052209: 89$ & HCG-9: 30054159 - 30056871 \\
\hline 29. & $245 C 6-3$ & T729 & 280 & & 30216316 / 216321 - 216669: 84 & TRIM40: $30212489-30223570$ \\
\hline 30. & 463 N5-3 & HLA-B32 & 332 & $\begin{array}{l}590551 \\
675100 \\
\end{array}$ & 31432914 / 432798 - 432580: 92 & $\begin{array}{l}\text { HLA-B: } 31429628-31432914 \\
\text { HLA-C: } 31347834-31347834\end{array}$ \\
\hline 31. & $463 N 5-3$ & ptarbac3 & 365 & & $31374599 / 374599-375006: 85$ & HCGII-3: 31376001 - 31379540 \\
\hline 32. & 436B4-3 & $\mathrm{T} 7.29$ & 273 & $\begin{array}{l}590608 \\
675178 \\
\end{array}$ & 31432914 / 429344 - 429048: 94 & $\begin{array}{l}\text { HLA-B: } 31429628-31432914 \\
\text { HLA-C: } 31347834-31347834\end{array}$ \\
\hline 33. & $325 \mathrm{P} 21-3$ & $\mathrm{~T} 7.29$ & 323 & & 30227701 / 231061 - 231414: 91 & TRIM10: $30227701-30236690$ \\
\hline 34. & $325 P 21-3$ & ptarbac3 & 171 & & 29903497 / 904397 - 904559: 94 & HLA-G: 29903497 - 29906859 \\
\hline 35. & $279 K 21-3$ & ptarbac3 & 482 & & 30611717 / $611549-611056: 98$ & HLA-E: 30565250 - 30569077 \\
\hline 36. & $224 \mathrm{H} 20-3$ & T7.29 & 159 & & & \\
\hline 37. & $348 C 21-4$ & T7.29 & 485 & & 29732788 / 746705 - 747350: 93 & MOG: 29732788 - 29748128 \\
\hline 38. & $171 \mathrm{~K} 8-4$ & T7.29 & 637 & & $29732788 / 743737-744314: 88$ & MOG: $29732788-29748128$ \\
\hline 39. & $171 \mathrm{~K} 8-4$ & ptarbac2 & 567 & & 29732788 / 743747 - 744316: 88 & MOG: 29732788 - 29748128 \\
\hline 40. & $171 K 8-4$ & Caja G & 362 & $\begin{array}{l}587515 \\
672050\end{array}$ & 31347834 / 347713 - 344005: 90 & $\begin{array}{l}\text { HLA-B: } 31429628-31432914 \\
\text { HLA-C: } 31347834-31347834\end{array}$ \\
\hline 41. & 239N18-4 & $\mathrm{T} 7.29$ & 319 & & $29732788 / 743775-744384: 88$ & MOG: $29732788-29748128$ \\
\hline 42. & 120D3-1 & HLA-B32B & 175 & $\begin{array}{l}590071 \\
674636\end{array}$ & 31429628 / 433575 - 433744: 89 & $\begin{array}{l}\text { HLA-B: } 31429628-31432914 \\
\text { HLA-C: } 31347834-31347834\end{array}$ \\
\hline 43. & 120D3-1 & HLA-B32B & 244 & $\begin{array}{l}590086 \\
674651\end{array}$ & 31429628 / $433593-433843: 89$ & $\begin{array}{l}\text { HLA-B: } 31429628-31432914 \\
\text { HLA-C: } 31347834-31347834\end{array}$ \\
\hline 44. & 120D3-1 & HLA-B29 & 235 & $\begin{array}{l}590874 \\
675422\end{array}$ & 31425377 / 425251 - 425012: 93 & $\begin{array}{l}\text { HLA-B: } 31429628-31432914 \\
\text { HLA-C: } 31347834-31347834\end{array}$ \\
\hline 45. & 120D3 -1 & Caja-G & 666 & $\begin{array}{l}587217 \\
671748\end{array}$ & 30334627 / 347882 - 348645: 85 & $\begin{array}{l}\text { HLA-B: } 31429628-31432914 \\
\text { HLA-C: } 31347834-31347834\end{array}$ \\
\hline
\end{tabular}




\begin{tabular}{|l|l|l|c|l|l|l|}
\hline 46. & $120 \mathrm{D} 3-1$ & Caja-E & 363 & $\begin{array}{l}587910 \\
672448\end{array}$ & 31347834 / 344583 - 344998: 88 & $\begin{array}{l}\text { HLA-B: 31429628 - 31432914 } \\
\text { HLA-C: } 31347834-31347834\end{array}$ \\
\hline 47. & 120D3-1 & MIC & 142 & & $30018310 / 011199-011349: 86$ & HLA-A: 30018310 - 30021633 \\
\hline 48. & 120D3-1 & Klex2 & 84 & & $30018310 / 018399-018502: 93$ & HLA-A: 30018310 - 30021633 \\
\hline 49. & 193 P12-1 & T7.29 & 693 & & No alignment in Hs localized & \\
\hline 50. & $18 G 14-1$ & ptarbac2 & 279 & & ptarbac2.1 = vector & \\
\hline 51. & $19116-1$ & T729 & 221 & & No alignment in Hs localized & \\
\hline 52. & 174 N1-3 & T7-29 & 202 & & ptarbac2.1 = vector & \\
\hline 53. & $169 G 2-1$ & T729 & 121 & & $31159820 / 159494-159368:$ & HCG22: 31157810 - 31159820 \\
\hline
\end{tabular}

1: Insertions in Callithrix jacchus omitted for calculation of Venter genome bp numbers

2: Insertions/deletions in Callithrix jacchus disregarded for calculation of \% identity with Hs

3: For Venter genome bp numbers of genes see map to bp-scale of the MHC class I region in Hs, figure 5.4.1.

The 55 sequences mapped and tabulated mostly belong to the intervalls MOG TCTEX4 and ATP6gV1 - POU5F1 (see 5.2.3.3.1 and 5.2.1.3.1) called for short contigs 4 and 1 , respectively.

The sequences of contig 4 were only localized and possibly gene assigned. Deeper considerations could be given only to some items of contig 1. Especially a number of sequences aligning in the HLA-B/C section were evaluated for their identity with a homologous part of the Hs B versus the $\mathrm{C}$ duplicon. As the results displayed in table 5.4.2 show, all of these HLA sequences by their alignment proved to be more similar to duplicon $\mathrm{B}$ rather than to $\mathrm{C}$.

On the other hand, TS1 is also aligning in both duplicons B and C at high identities. With its unique and absolutely rare insertion of a rather young ferritin pseudogene (see below), a TS1 containing this rarity showed have been sequenced from one of two probable sequences, either the $\mathrm{B}$ or in the $\mathrm{C}$ duplicon. Because of a high identity in these duplicons, a critical evaluation is required.

To avoid a background of mutations irrelevant for the decision, only those point mutations were counted that affected only one stretch either B or C. Most of the mutations between TS1 and its homologous sequences in Hs and Patr duplicons B and $\mathrm{C}$ were disregarded. In other words the similarties in these crucial bases between TS1 and either strand B or C were counted. Table 5.4.3 shows the results: generally higher similarities with duplicon $C$ in all three partial sequences of TS1-ps1, -ps2 and - $v$ fragments in both Hs and Patr (see 5.6.1). Therefore, TS1 seems to be a homologous copy sequenced from BAC clone DNA stemming from a Caja duplicon C. However, more homologues of TS1-pseudogenes ps1, ps2 and v shall be compared, e.g. those of the 14 Mamu-B duplicons; results will be assed by statistical evaluation. A total sequencing of the HLA B/C section started by Prof. Takashi Shiina should present the final answer soon. 
From these results i.e. the sequences localized near HLA-B/C all having higher identities with Hs duplicon $B$, and TS1 having more specific similarities with Hs duplicon C, we may conclude that Callithrix jacchus has both duplicons, B and C, in agreement with Hs, Patr and other primates (see below, 5.6.2 and Discussion, 6.4). In conclusion, these sequences within the for time available could all be defined, but evaluated only in some parts seeming of more general interest, as for their evolution (5.5), TS1, however, for its special challenges for molecular immunogenetics (5.6).

\section{Table 5.4.1.2: Similarity of TS1-ps1, TS1-ps2 and TS1-v with homologous DNA} in duplicons $\mathbf{B}$ and $\mathbf{C}$ of $\mathrm{Hs}$ and Patr. For lowering background by not counting mutations occurred just in TS1 or even in all three strands, only single base exchanches in either B or C have been compared with TS1 bases that rement unchanged, i.e. equal with one base in either strand $\mathrm{C}$ or $\mathrm{B}$. The procedure allows to better weigh relative similarity of TS1 with both duplicons, B and C. Further data should be collected, e.g. from the 14 Mamu-B duplicons in order to have greater numbers for a statistical evaluations. These preliminary data favor TS1 being taken from a Caja duplicon $\mathrm{C}$.

\begin{tabular}{|l|c|c|c|}
\hline Mutation points rement equal in & TS1-ps1 & TS1-ps2 & TS1-v \\
\hline Hs duplicon B & 35 & 19 & 2 \\
\hline Hs duplicon C & 36 & 31 & 9 \\
\hline Patr duplicon B & 34 & 18 & 5 \\
\hline Patr duplicon C & 40 & 29 & 9 \\
\hline
\end{tabular}

\subsubsection{Collection and presentation of sequence data}

Nearly all of the 55 pilot sequences obtained by PCR on Caja BAC clones have been localized and where possible gene assigned by comparative alignment in the Hs genome. These results are shown in a map to bp-scale, Figure 5.4.1.1. The alignment data of the sequences were calculated in bp numbers both on the scale by T. Shiina and of the Venter genome. The alignment was defined by first and last aligning bp and shown in table 5.4.1.1

The results of localization are visualized in the map shall be completed with all genes known in the Hs MHC class I region. All loci of the class I genes in Hs, and for comparison those of the Callithrix jacchus sequences found, have been defined and listed in table 5.4.1.1. 
The data of localization and assignment by homologous alignment in the genomes of primates and other mammals are collected in table 5.4.2.

\subsubsection{Demonstration of individual sequences identified by localization and}

\section{possibly gene assignment in the Hs genome or proteome sequence K19161C12-TS2}

This sequence contains $3000 \mathrm{bp}$. The AUGUSTUS program indicated a gene between positions 236 and 2259. The alignment was compatible with BAT1 gene in human (Allcock et al., 1999). The total sequence aligned. In the BAT1 gene, it is from intron 4 until intron 6, see Figure 5.4.2.

BAT1HS

K19_161C12TS2

Clustal Consensus

BAT1HS

K19 161C12TS2 Clustal Consensus 68

BAT1Hs Clustal Consensus 151

BAT1Hs

K19_161C12TS2

Clustal Consensus

BAT1HS

K19_161C12TS2

Clustal Consensus

BAT1Hs

K19 161C12TS2

Clustal Consensus

BAT1HS

Clustal Consensus 501

BAT1HS

K19_161C12TS2

Clustal Consensus

BAT1Hs

K19_161C12TS2

Clustal Consensus

BAT1HS

K19 161C12TS2

Clustal Consensus

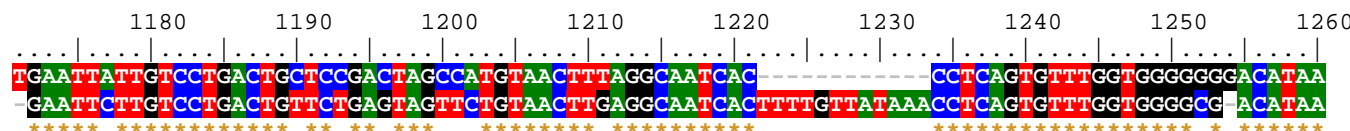

$\begin{array}{rrrrrrrrr}1270 & 1280 & 1290 & 1300 & 1310 & 1320 & 1330 & 1340 & 1350\end{array}$ AATTTTTAAATTAGGTGACCTCTAAA-GTTAGTTTTAGTTTGGAACATGCTAAAAATTGGTTAGCTCAAAC -AGAGTGGACCCTG AATTTTTAAATTAGG TGACCTCTAAAAGTTTATTGTAGTTTTGAAACATGCTAAAAATTGGTTTAGCTCAAACTGAGAGTGGGAACCCTG

$\begin{array}{rrrrrrrrr}1360 & 1370 & 1380 & 1390 & 1400 & 1410 & 1420 & 1430 & 1440\end{array}$

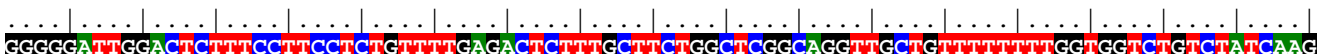
GGGGGATTGGACTCTTTCCTTCCTTGTTTTTGAGACTCTTTGCTCTTGGCTTGGCAGGTTGCTGTGTTTTTTGGTGGTCTGTCTATCAAG

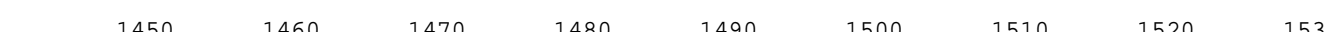

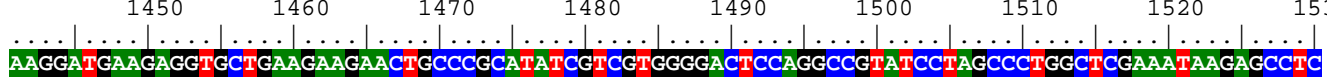
AAGGATGAAGAGGTGCTGAAGAAGAACTGCCCGCATATCGTCGTGGGGACTCCAGGCCGTATCCTAGCCCTGGCTCGAAATAAGAGCCTC

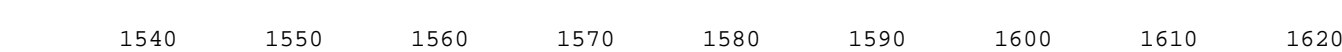
AACCTCAAACACATTAAACACTTTATTTTGGATGAATGTGATAAGATGCTTGACAGCTCGGTGAGTGGCAGTGCTGGGGCTTGGCTAAT AACCTCAAACACATTAAACACTTTATTTTGGATGAATGCGATAAAATGCTTGAACAGCTCGGTGAGTGGCAGTGCTGGGGCTTGGCTAAT

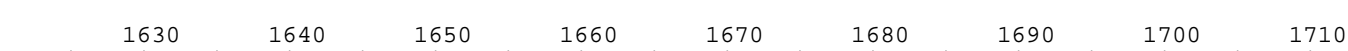

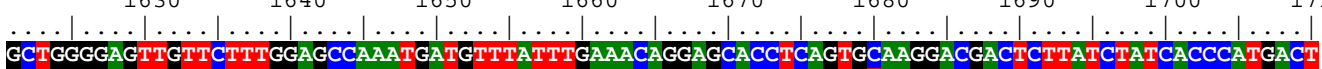
GCTGGGGAGTTGTTCTTTGGAGCCAAATGATGTTTATTTGAAACAGGAGCACCTCAGTGTAAGGACGACTCTTATCTATCACCCATGACT \begin{tabular}{|l|l|l|l|l|l|l|l}
1720 & 1730 & 1740 & 1750 & 1760 & 1770 & 1780 & 1790
\end{tabular} GATGGCTCTGGGTCCCTGGTTGGTCTTTATTATGCTTTTAAGCACAGTAAGGGTGTCATCTATCATCTTTCTATGATTTTTGTTTTTA GATGGCTCTGGGTTCCTGGTTGGTTTTATT- - CTTTTAAGCACAGTAAGGG GTCATCTATCATCTTTCTATGATTTC- ITTTTA

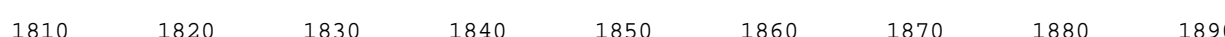

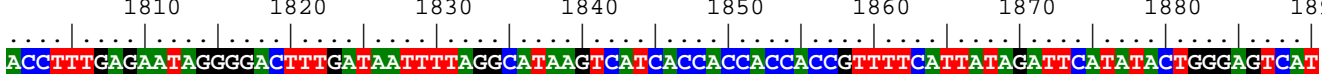

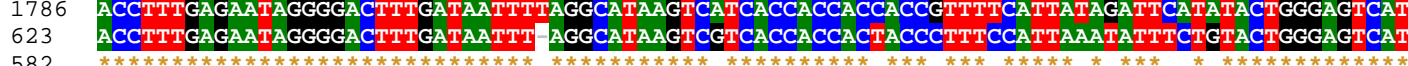

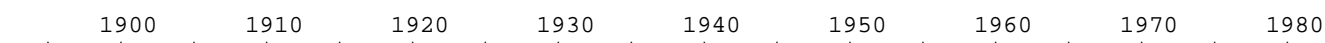
AGGGGAGATTCTAAACTGAAAGAGAAGACAGT-ACCCTTCTGGCATCTCCAGCACAGCATTTACAGTCAGAATTTATAGCTGATATAGT AGGGGATATTCTAAACTGAAGGAGAAGACTGTCTACCCTTATGGCATCTCCAGCACAACATACACAGTCAGAATTTATACCTGAATCAGT

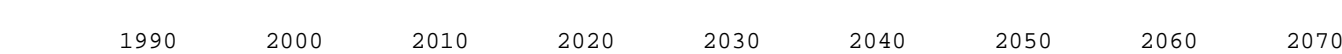
GTCTAGACTCAGGTCTGGGTTAATGTAGAGAGTGTTTGTAGCAGTTTGTGTGATGTGGTATTCTAGTGTGCAGGTGGGTTATGGAITCTAGACTCGGGTCTGAAATTAATGTAGAGAGTGTTTGTAGGAGTTCATGTGGTGTGGTATTCTAGT- GCTAAGTGGGTTAATGAAG 
BAT1HS

K19_161C12TS2

Clustal Consensu

BAT1HS

K19_161C12TS2

Clustal Consensus

BAT1Hs

K19_161C12TS2

Clustal Consensus

BAT1Hs

K19 161C12TS2

Clustal Consensus

BAT1Hs

K19 161C12TS2 Clustal Consensus 1129

BAT1HS

K19_161C12TS2

2491

Clustal Consensus 1179

BAT1Hs

1384

Consensus

BAT1HS

K19 161C12TS2

Clustal Consensus

BAT1Hs

K19 161C12TS2

Clustal Consensus

BAT1HS

Clustal Consensus 1509

BAT1Hs

K19_161C12TS2

Clustal Consensus

BAT1Hs

K19 161C12TS2

Clustal Consensus

BAT1HS

K19 161C12TS2

Clustal Consensus

BAT1Hs

K19 161C12TS2

Clustal Consensus

BAT1HS

K19_161C12TS2

clustal Consensus
2120

$2130 \quad 2140$

2150

2160

$\ldots|\ldots| \ldots|\ldots| \ldots|\ldots| \ldots|\ldots| \ldots|\ldots| \ldots|\ldots| \ldots|\ldots| \ldots|\ldots| \ldots|\ldots| \ldots|\ldots| \ldots \mid$ AAGATTTTTCTGTCAGAATTGAATCTTGGTAAATGAGAGTGGGGATGGACATAGGCTCCAGAGAAAAGTTATTACAAATGATCTTTGGC

$\begin{array}{lllllllll}2170 & 2180 & 2190 & 2200 & 2210 & 2220 & 2230 & 2240 & 2250\end{array}$

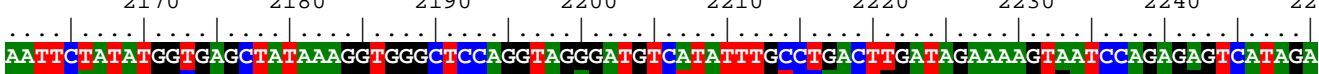
ATTCAATATGGCGAGCTATAAAGGTGGGCTCCAGGTAGAGATGTCTTATTTGTCCAACTGGATAGAAAAGTGATCCAGAGGGTCATAGA

$\begin{array}{lllllllll}2260 & 2270 & 2280 & 2290 & 2300 & 2310 & 2320 & 2330 & 2340\end{array}$ $\ldots \ldots|\ldots| \ldots|\ldots| \ldots|\ldots| \ldots|\ldots| \ldots|\ldots| \ldots|\ldots| \ldots|\ldots| \ldots|\ldots| \ldots|\ldots| \ldots|\ldots| \ldots \mid$ IGAACTTTGATAGCTGGAATGTAATATGTGCTTGATATTTGTAGTCTGCTGAAGGCTGGCCTGGGCTTGGGCAGGAAAGAGTTGGGAGAA

$\begin{array}{lllllllll}2350 & 2360 & 2370 & 2380 & 2390 & 2400 & 2410 & 2420 & 2430\end{array}$ $\ldots \ldots|\ldots| \ldots|\ldots| \ldots|\ldots| \ldots|\ldots| \ldots|\ldots| \ldots|\ldots| \ldots|\ldots| \ldots|\ldots| \ldots|\ldots| \ldots|\ldots| \ldots \mid$ TGGCCTGTAAAGCATGTTTTGAAGGCCTTATGCCTGAGAGTATCTGCACAGGGCTTTGTCCCCATTTTATAGTTGTGAACTTCGGGGTTT

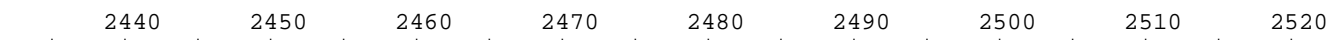

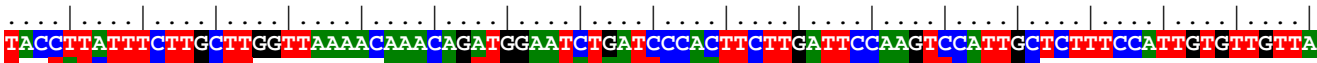
TATCTTTCTTGCTT-

$\begin{array}{lllllllll}2530 & 2540 & 2550 & 2560 & 2570 & 2580 & 2590 & 2600 & 2610\end{array}$ CTATTTCCAGCAATCTTCACCTCACTGGGAAGTCTACCTCTAATCTTTGTTTATCATACCTGCTTATTTTCTCCTACAATTTTTTTCCTT AGTCTTCACCTCACTGGGAGGTCACCTTTAATCTTT- - ACTATACCTGCTTATTTTCTCCTACAATTCTTTTC-TT

$\begin{array}{lllllllll}2620 & 2630 & 2640 & 2650 & 2660 & 2670 & 2680 & 2690 & 2700\end{array}$ $\ldots|\ldots| \ldots|\ldots| \ldots|\ldots| \ldots|\ldots| \ldots|\ldots| \ldots|\ldots| \ldots|\ldots| \ldots|\ldots| \ldots|\ldots| \ldots|\ldots| \ldots|\ldots|$ GTTCTCATAGACATGCGTCGGGATGTCCAGGAAATTTTTCGCATGACCCCCCATGAGAAGCAGGTCATGATGTTCAGTGCTACCTTGAGC $\begin{array}{lllllllll}2710 & 2720 & 2730 & 2740 & 2750 & 2760 & 2770 & 2780 & 2790\end{array}$ AAAGAGATCCGTCCAGTCTGCCGCAAGTTCATGCAAGATGTAAATACCCTTCTACCTTCTCTCCCTCCACTCCCCGCCCCTGCCTCCTC AAAGAGATCCGTCCAGTCTGCCGCAAGTTCATGCAAGATGTAAATACCCTTCTACCTTCTCTCCCTCCACTCCCCGCCCGCTGCCTCCTC

$\begin{array}{rrrrrrrrr}2800 & 2810 & 2820 & 2830 & 2840 & 2850 & 2860 & 2870 & 2880\end{array}$ $\ldots|\ldots| \ldots|\ldots| \ldots|\ldots| \ldots|\ldots| \ldots|\ldots| \ldots|\ldots| \ldots|\ldots| \ldots|\ldots| \ldots|\ldots| \ldots|\ldots| \ldots \mid$ CCCTTCCTCGCCCTCTTCCTCCAGACTCCCTTGTCATTCAAGT GCCAAGAAGTCGGCTTATGCCCAGCTGGGAGTAACGACTCCTTGAAG

$\begin{array}{lllllllll}2890 & 2900 & 2910 & 2920 & 2930 & 2940 & 2950 & 2960 & 2970\end{array}$ AGACATACAGAAGCAGAGACAGCTAGTGTTAGGGCCTGCGCGGGTGCCAGGGAACTCCGGAAGACTTGGTCGGGTTAATGTGAGAGCGG AGACATACAGAAGCAGAGACAGCTAGTGTTAGGGTCTGCGCGGGTGCCAGGGAAACTCCGGAAGACTTGGTCGGGTTAATGTGAGAGCGG

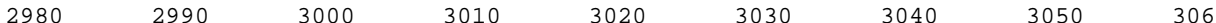
GTAGTGTTCGACTTTTTC 1744 GTAGTGTTCGACTTTTTCTCAAAAATCGCAACATTTTTGAACCTCTTCTCCTTTTGGGGGAGGCAGGATTTTTCTGCCCTACCACCCAC

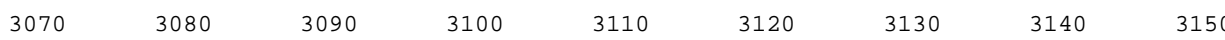

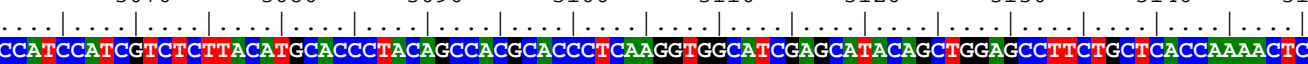
CCATC- - GTGTCTTACGTGCCCCCTACAGCCACGCATCCTCAAGGTGGCATCGAGCATACAGCTGGAGCCTTCTGCTCACCAAAACTC

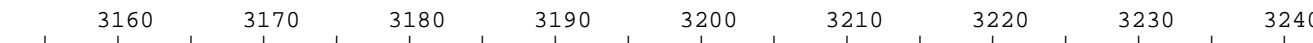
CTACTTCCCGGTGGCAGGAGAGCAAGAGAGGGACAGACAGATGGCAGGGCATGTCCAAAAGAAGAGCATCAGCACAAATGAATCCTCCCC CTACTTCCCAGTGGCAGGAGAGCAAGAGAGGGACAGACAGATGGCAGGGCATGTCCAAAAGAAGAGCATCAGCACAGATGAATCCTCCCC

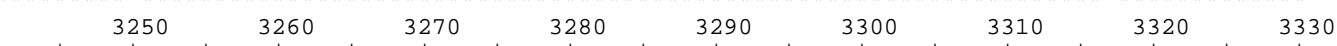
$\ldots|\ldots| \ldots|\ldots| \ldots|\ldots| \ldots|\ldots| \ldots|\ldots| \ldots|\ldots| \ldots|\ldots| \ldots|\ldots| \ldots|\ldots| \ldots|\ldots|$ TTCCCCACCT CCAGGGG TGGGGCCTTTGGCACCTCAATCCCCGATACCCTACTCCTTCCCACCCACATCTCCTTGCACCCATCCGGAAC

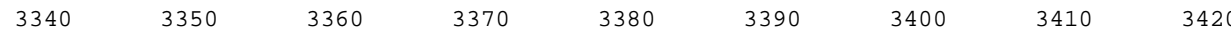
CTCGGTTGATGTGAGCCGGCACAGAGAAGCACCGTGGCGGGCGAGGAATGCAGACGGCACCAGCGGTGGATGGCGCAGCGGAGGC CTCGGTTGATGTGAGCCGGCAACAGAGAAGCACCGT GGCGCGGCGAGGGATGCGGACGGCACCCAGCGGTGGATGGCGGCAGCGGAGGC 


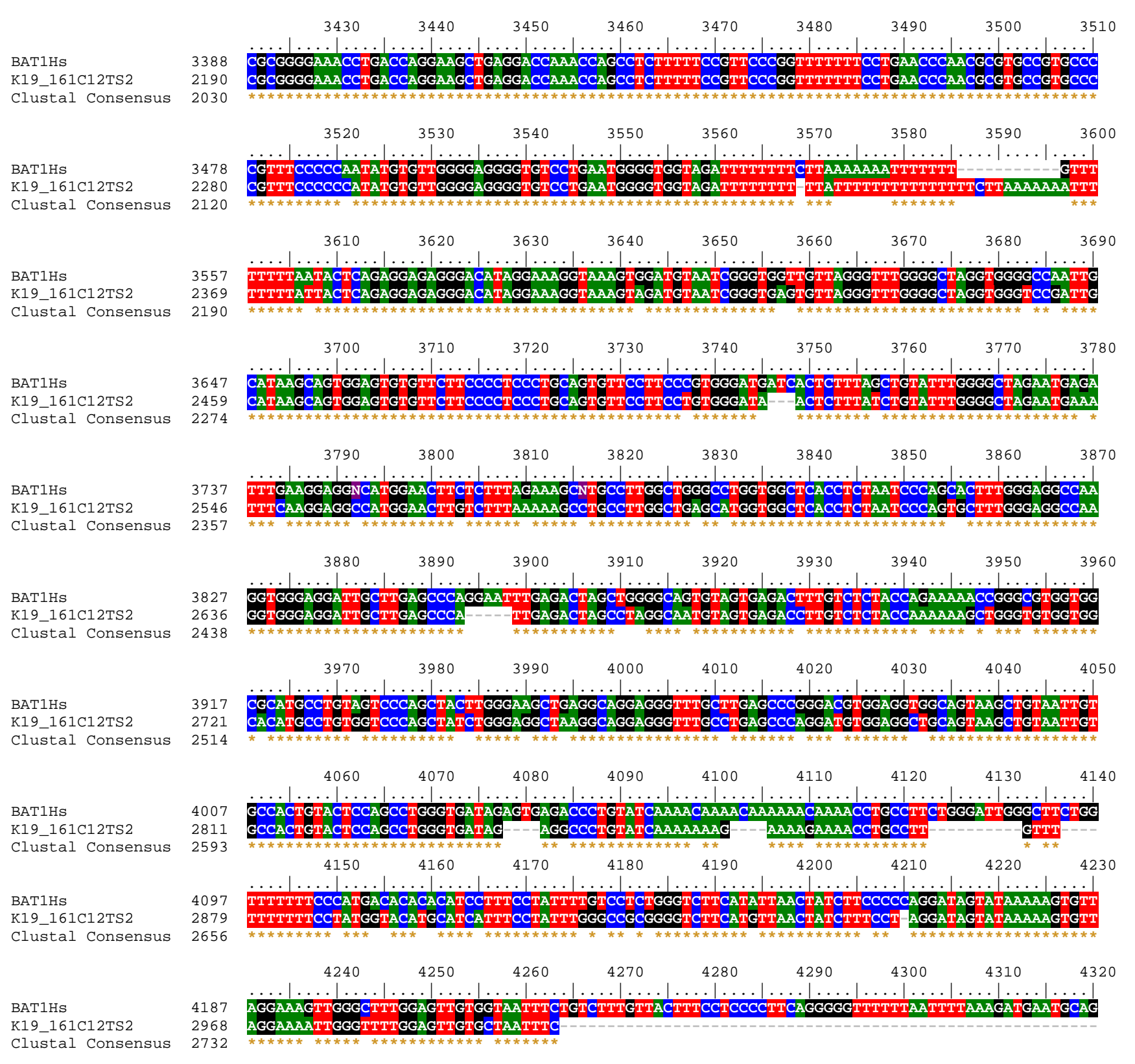

Figure 5.4.2: K19-161C12-TS2: part of BAT1 gene

Alignment has been performed between K19-161C12-TS2 and BAT1 gene of Hs (ID:NM 004640) by Clustal X, into intron 4 until 6 in position 1172 until 4263.

\subsubsection{K28-347D1-TCF19, nucleotide sequence}

This sequence contains $2314 \mathrm{bp}$. The AUGUSTUS program indicated a gene between positions 532 and 1968. The alignment was compatible with a part of gene TCF19, however, the nucleotide sequence showed only a small fragment high identity (see Figure 5.4.2.1). Nevertheless, this sequence was translated by Swissprot program; thereupon the amino acid sequence was aligned in Clustal $X$. The sequence had a homology with the human sequence between positions 158 and 
239, (see Figure 5.4.2.2) again only a small part of the whole gene product (protein). Although, such homology may deserve consideration, e.g. from a comparative evolutionary aspect, we have generally refused such finding as being in sufficient for both localization and gene assignment of a sequence.

K28_347D1TCF19_10 1 TCF19Shiina 451 Clustal Consensus 1

K28_347D1TCF19_10 16 TCF19Shiina Clustal Consensus 13

K28_347D1TCF19_10 104 TCF19Shiina Clustal Consensus 90

K28_347D1TCF19_10 194 TCF19Shiina 719 Clustal Consensus 173

K28_347D1TCF19_10 284 TCF19Shiina 809 Clustal Consensus 253

K28 347D1TCF19_10 349 TCF19Shiina 899 Clustal Consensus 298

K28 347D1TCF19 $10 \quad 386$ TCF19Shiina 989 Clustal Consensus 325

K28_347D1TCF19_10 458 TCF19Shiina Clustal Consensus 368

K28_347D1TCF19_10 519 TCF19Shiina 1167 Clustal Consensus 402 $\begin{array}{lllllllll}460 & 470 & 480 & 490 & 500 & 510 & 520 & 530 & 540\end{array}$ GCCCCTCGGAGTTCTACTTCATGTTCCAACAAGTACGAGTCAAGCCTCAGGACTTTGCT GCCATTACCATCCCACGGTCTAGGGGAGAAG

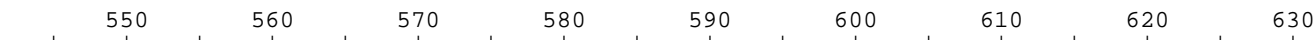
G $|\ldots| \ldots|\ldots| \ldots|\ldots| \ldots|\ldots| \ldots|\ldots| \ldots|\ldots| \ldots|\ldots| \ldots|\ldots| \ldots|\ldots| \ldots|\ldots| \ldots \mid$ CCCGGGTTGGGGTGGTTTCCGGCCTATGCTGCCCTCCCAGGGGCTCCACAG--CGGCCTCTCAGCACCTTCTCCCCTGCCCCCAAGGC

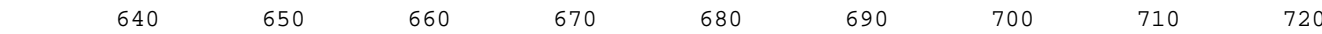

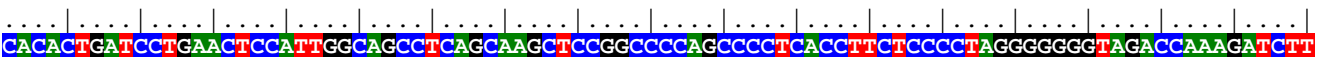
CACACTGATCCTAAACTCCATAGGCAGCCTCAGCAAGCTCCGGCCCCAGCCCCTCACCTTCTCCCCTAGTTGGGGTGGACCAAAGAGCCT
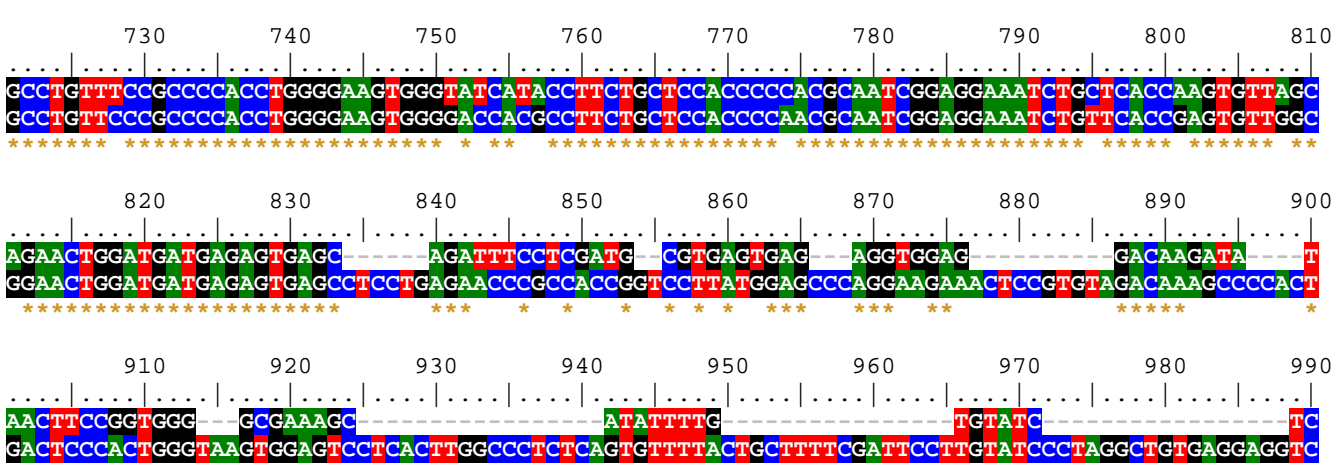
$\begin{array}{rrrrrrrrr}1000 & 1010 & 1020 & 1030 & 1040 & 1050 & 1060 & 1070 & 1080\end{array}$ $\ldots|\ldots| \ldots|\ldots| \ldots|\ldots| \ldots|\ldots| \ldots|\ldots| \ldots|\ldots| \ldots|\ldots| \ldots|\ldots| \ldots|\ldots| \ldots|\ldots| \ldots|\ldots|$ CCCCTGCCTGGGGGATGGGCACGGGAGGTGGAATAGATGGAATGGCAAGACCTGGGTTAGCTCTG--ATAGGAAAAGAAAAATATGTGC

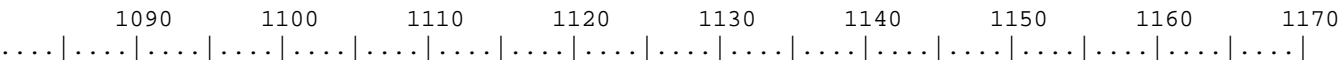

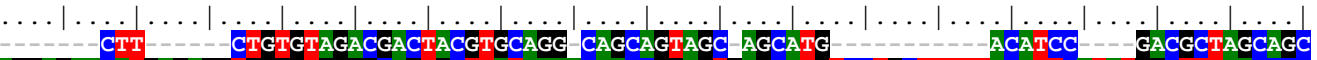
7 AGGAGACATGAGAGGTGGGTGGGGAGTGCTTATAAAACAACCGGAGTGAGCATGTCCTGCTTTTTACATTCATATGGCTTTAACCCC

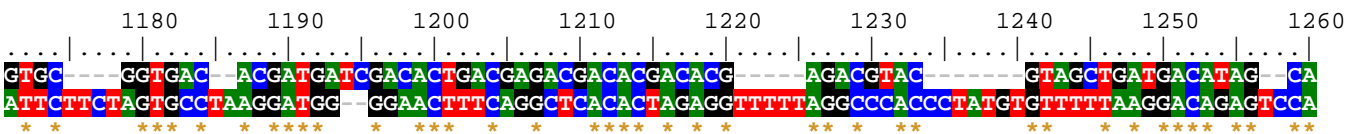

K28_347D1TCF19_10 589 Clustal Consensus 444

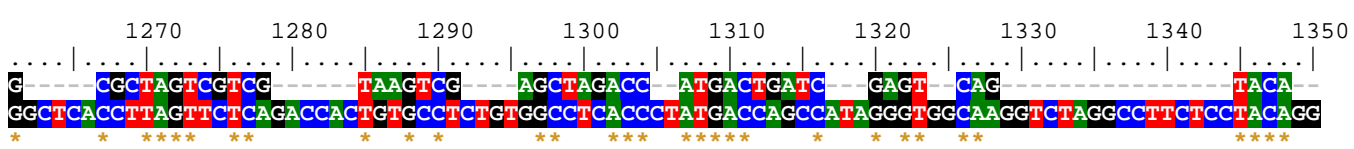

Figure 5.4.4.1: K28-347D1-TCF19, nucleotide sequence

Alignment has been performed between K28-347D1-TCF19 and TCF19 gene of Hs (ID: NM 001077511) by Clustal X, in position 460 until 1350. 
K28_347D1TCF19_10 1 HsTCF19

Clustal Consensus 1 KSTCF19 Clustal Consensus 11

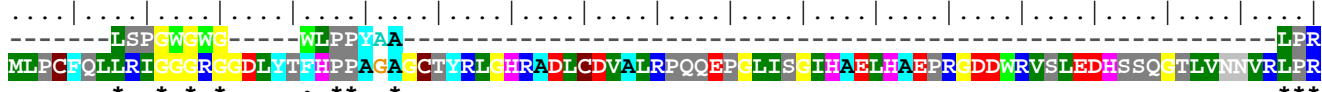

$\begin{array}{rrrrrrrrr}100 & 110 & 120 & 130 & 140 & 150 & 160 & 170 & 180\end{array}$ $\ldots|\ldots| \ldots|\ldots| \ldots|\ldots| \ldots|\ldots| \ldots|\ldots| \ldots|\ldots| \ldots|\ldots| \ldots|\ldots| \ldots|\ldots| \ldots \mid$

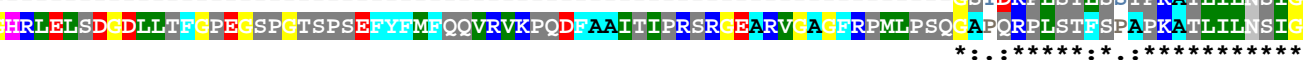

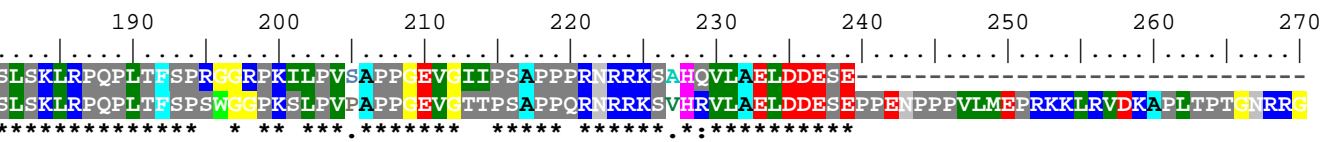

K28_347D1TCF19_10 HSTCF19

Clustal Consensus 34
320

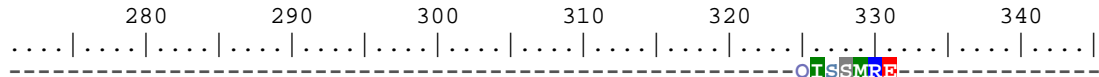

K28_347D1TCF19_10 $101 \ldots$

HsTCF19 271

Clustal Consensus 83
RPRKYPVSAPMAPPAV EPCAAPCCLPQEETVAWVQCD CDVWFHVACV CSIQAAREADFRCP CRA IQT

Figure 5.4.4.2: K28-347D1-TCF19, amino acid sequence

Translated nucleotide sequence was performed with AGUSTUS and BLAST. Alignment has been performed between K28-347D1-TCF19 and TCF19 protein of Hs (ID: NM 001077511) by Clustal X, the homology is localized in position 157 until 239.

\subsubsection{MOG sequences}

K24-171K8ptarbac2-38 and K24-171K8ptarbac2-39 were compatible alignment with MOG gene. The human MOG gene had a length of 15340 with eight exons (ID: 4340).

K24171K8ptarbac2-38 sequence presented a homology $86 \%$ in the position 10933 until 11573, with four insertions and two deletions, this region corresponds to intron 1-2 (89-8942), see Figure 5.4.5.1.

K24171K8ptarbac2-39 sequence presented a homology $88 \%$ in the position 6010 until 6579, with 3 insertions and 3 deletions, this region corresponds to intron 1-2 (898942), see Figure 5.4.5.2.

Both sequences not presented homology with MOG gene sequence from Callitrhix jacchus (gene ID: AY566834).

K24_171K8T729_38 1 MOGHS Clustal Consensus 1

K24_171K8T729_38 1 MOGHS

Clustal Consensus 1

K24_171K8T729_38 48 MOGHS Clustal Consensus 37

\section{$\begin{array}{lllllllll}10810 & 10820 & 10830 & 10840 & 10850 & 10860 & 10870 & 10880 & 10890\end{array}$} 10801 AGCAGGAAGAGTCTTCACTCCTCCCAATGCCCACCTCCCATTTGTTACTAAGAGGAGAGGAGAAAGTAGCAAGGAGGGTATGGGATG $\begin{array}{lllllllll}10900 & 10910 & 10920 & 10930 & 10940 & 10950 & 10960 & 10970 & 10980\end{array}$

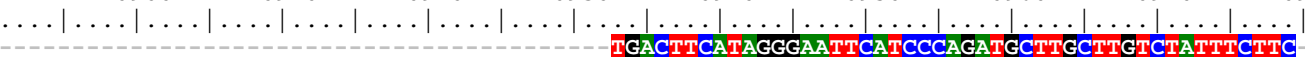
10891 TTCTGGGGAATGGG GTTGGTGCATCAACAACAAAGTCCTTTCTCTCACCTTGAATTCATCCCAGATGCCTGCTTGTTTACTTCTTCC

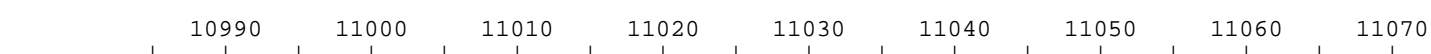
ACATGAAAA- $\ldots|\ldots| \ldots|\ldots| \ldots|\ldots| \ldots|\ldots| \ldots|\ldots| \ldots|\ldots| \ldots|\ldots| \ldots|\ldots| \ldots|\ldots| \ldots|\ldots| \ldots \mid$ ACACAAAAAAAGGCCTTCAGCCCTCATGGCTGAGCAGAAAGAATCTGAATGTTAGAGTCAGGCAGCCTGGGTTTGAATTCCATCTCAGGT 
\begin{tabular}{|l|llllllll}
\hline 11080 & 11090 & 11100 & 11110 & 11120 & 11130 & 11140 & 11150 & 11160
\end{tabular} K24_171K8T729_38 $137 \quad$ ACC - ACACTATGGCAAATTCTTAGATTCTCCAAGCCTCAGTTGCCTTGTCTGCCAAATAGAGAACACATCCTCCCTCCTAAATTGTAA $\begin{array}{lll}\text { K24_171K8T729_38 } & 137 & \text { ACC--ACACTATGGCAAAATTCTTAGATTCTCCAAGCCTCAGTTGCCTTGTCTGCCAAATAGAGAACACATCCTCCCTCCTAAATTGTAA } \\ \text { MOGHS } & 11071 \text { ACTGAACTCTATAGCAAAATTCTTAGATTCTCCAAGCTTCAGTTGCCTTGTCTGTCAAATAGAGAAAACATCCTTCGTCCTAAATTGTAG }\end{array}$ Clustal Consensus 118

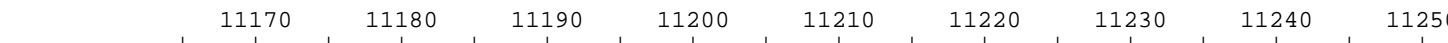

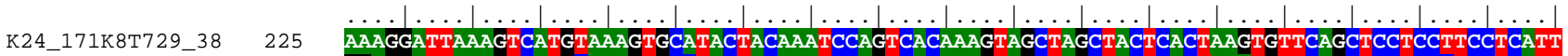

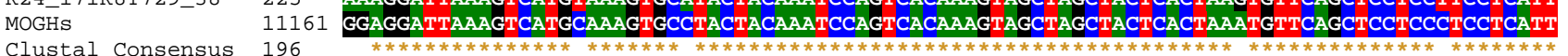

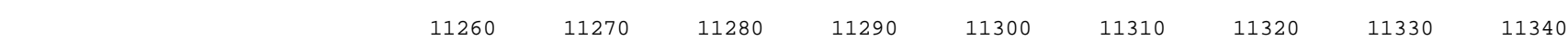
K24_171K8T729_38 $315 \quad \ldots \ldots|\ldots| \ldots|\ldots| \ldots|\ldots| \ldots|\ldots \ldots| \ldots|\ldots \ldots| \ldots|\ldots| \ldots|\ldots| \ldots|\ldots| \ldots|\ldots| \ldots|\ldots| \ldots \mid$

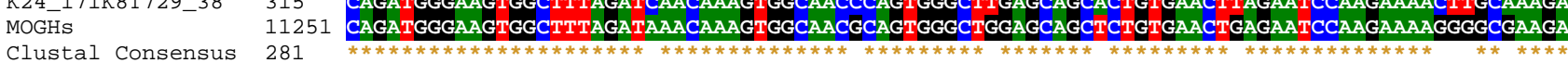

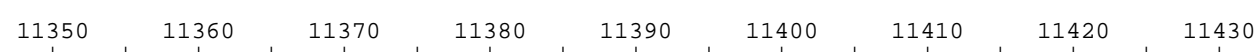
K24_171K8T729_38 405 GCAGCTGGGAGGTAGTAGATCCTTGTGTTGCTTTTGGAATTGTTCACATTCTTTATTCACCTATTGAATCTAGACTCTAGCTAGAGA

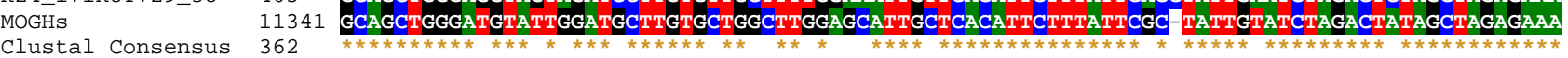

K24_171K8T729_38 495 MOGHS 11430 Clustal Consensus 436

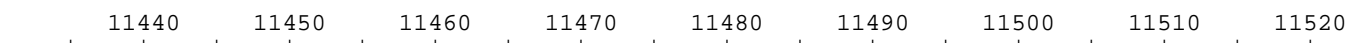
$\ldots \ldots|\ldots| \ldots|\ldots| \ldots|\ldots| \ldots|\ldots| \ldots|\ldots| \ldots|\ldots| \ldots|\ldots| \ldots|\ldots| \ldots|\ldots| \ldots, \ldots . \ldots|\ldots|$ GAGCCGCAACCATTGGCTTTAAATCCAGTGCTCTTCCTACTCTCCT-GAGGTTGTTTCCAGGCTGCAGAGAAATAGCCTGCACAAGGGGC

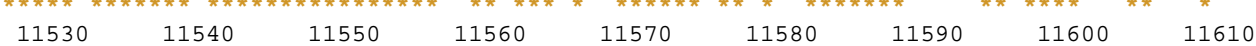
K24_171K8T729_38 $585 \quad \ldots \ldots|\ldots| \ldots|\ldots| \ldots|\ldots| \ldots|\ldots| \ldots|\ldots| \ldots|\ldots| \ldots|\ldots| \ldots|\ldots| \ldots|\ldots| \ldots|\ldots| \ldots \mid$

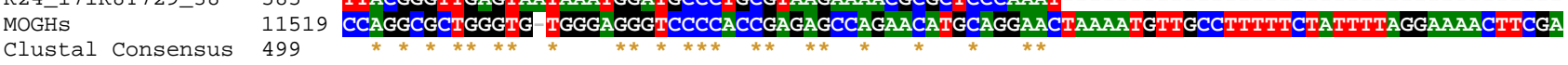

$$
\begin{array}{lllllllll}
11620 & 11630 & 11640 & 11650 & 11660 & 11670 & 11680 & 11690 & 11700
\end{array}
$$

K24_171K8T729_38 637 $\begin{array}{ll}\text { MOGHs } & 11608 \\ \text { Clustal Consensus } 522\end{array}$

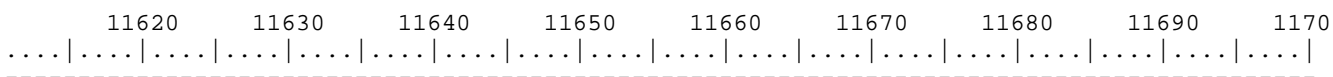

\section{Figure 5.4.5.1: Sequence K24_171K8ptarbac2_38}

Alignment has been performed between K24_171K8ptarbac2_38 and MOG gene of Hs (ID: AC000049) by Clustal X, into intron 1-2 in position 10933 until 11573.

K24 171K8ptarbac2 $39 \quad 1$ MOGHsrev Clustal Consensus 1

K24_171K8ptarbac2_39 22 MOGHsrev Clustal Consensus 9

K24_171K8ptarbac2_39 112 MOGHsrev

Clustal Consensus

K24_171K8ptarbac2_39 202 MOGHsrev

Clustal Consensus

K24_171K8ptarbac2_39 MOGHsrev

Clustal Consensus

K24_171K8ptarbac2_39 MOGHsrev

Clustal Consensus 79 149 292 6298 233

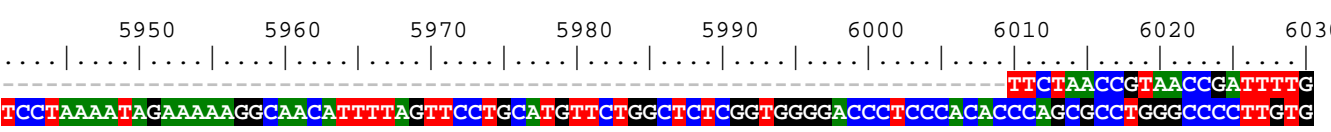

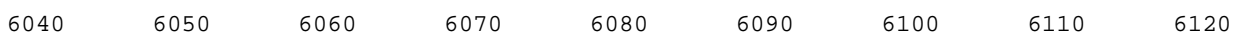

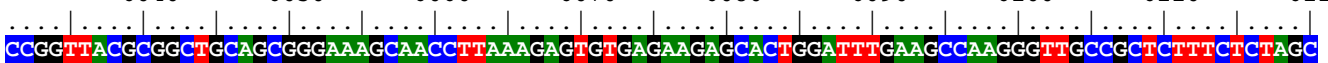
CAGGCTATTTCTCTGCAGCCTGGAAACAACCTCAG-GAGAGTAGGAAGAGCACTGGATTTAAAGCCAATGGTTGCGGCTCTTTCTCTAGC

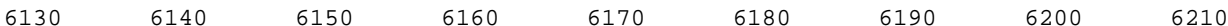
$\ldots \ldots|\ldots| \ldots|\ldots| \ldots|\ldots| \ldots|\ldots| \ldots|\ldots| \ldots|\ldots| \ldots|\ldots| \ldots|\ldots| \ldots|\ldots| \ldots|\ldots| \ldots|\ldots|$ TATAGTCTAGATACAATAG-CGAATAAAGAATGTGAGCAATGCTCCAAGCCAGCACAAGCATCCAATACATCCCAGCTGCTCTTCGCCCC

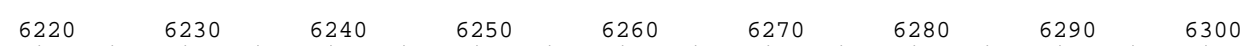
$\ldots \ldots|\ldots| \ldots|\ldots| \ldots|\ldots| \ldots|\ldots| \ldots|\ldots| \ldots|\ldots| \ldots|\ldots| \ldots|\ldots| \ldots|\ldots| \ldots|\ldots| \ldots \mid$ ITTTCTTGGATTCTCAGTTCACAGAGCTGCTC-CAGCCCACTGCGTTGCCACTTTGTTTATCTAAAGCCACTTCCCATCTGAATGAGGAG

$\begin{array}{lllllllll}6310 & 6320 & 6330 & 6340 & 6350 & 6360 & 6370 & 6380 & 6390\end{array}$

GGAGGAGCTGACACTTAGTGAGTAGCTAGCTACTTTGTGACTGGATTTGTAGTATGCACTTTACATGACTTTAATCCTTTTTACAATT GGAGGAGCTGAACATTTAGTGAGTAGCTAGCTACTTTGTGACTGGATTTGTAGTAGGCACTTTGCATGACTTTAATCCTCCCTACAATTT $\begin{array}{lllllllll}6400 & 6410 & 6420 & 6430 & 6440 & 6450 & 6460 & 6470 & 6480\end{array}$ 


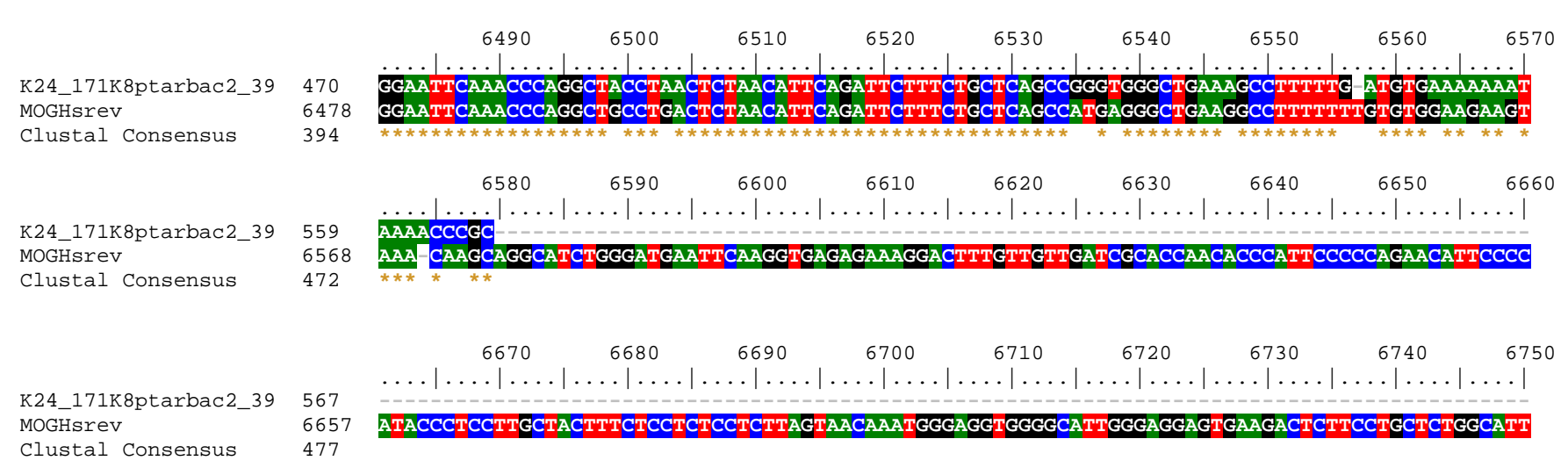

\section{4.5.2 Sequence 24_171K8ptarbac2_39}

Alignment has been performed between K24_171K8ptarbac2_39 and MOG gene of Hs (ID: AC000049) by Clustal X, into intron 1-2 in position 6010 until 6579.

\subsection{Evolution of class I sequences and framework genes found in Callithrix} jacchus compared to their homologous in other primates and mammals

The molecular evolution of four Callithrix jacchus genes is inspected here by gene trees, two for the Caja-G genes and comparison of Caja-G with the class la and class Ib HLA genes, and the one for the framework genes BAT1 and TCF19 as hit by the sequencing (Figures 5.5.1 - 5.5.5). Several other gene trees for the evolution of genes found in the Caja sequence TS1 are shown in the chapter 5.6 as TS1.

When selecting the data for computer calculations, we tried to insist on the principle of comparing only homologous parts of the sequences, both of Calithrix and other animals. Sequences were aligned with the help of the Clustal X program (Thompson et al., 1994) and thereupon used to make a phylogenetic tree including other animals that had shown identity in BLAST. The trees were constructed by the neighborjoining method (Saitou and Nei, 1987) using the MEGA version 3.1 program (Kumar et al., 20001) employing Tamura and Nei's model. The reliability of each node was assessed by the bootstrap method (Felsenstein, 1985) using 1000 replications.

\subsubsection{Gene trees of class I genes}

The sequence of K1:120D3Caja-G:45 and the sequences of Caja-G 01, 03, 04 and 05 as reported by Cadavid et al. (1997) are compared. The tree shows that Caja-G's genes formed a cluster. One gene the sequence K1-120D3-CajaG45, seems to represent the most ancient branch, closest to Caja-G4. It fell outside of this cluster during their evolution. 


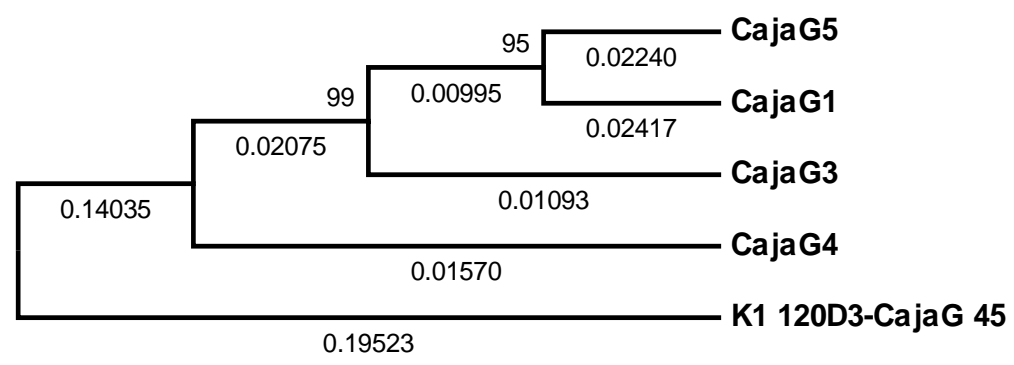

Figure 5.5.1.1: Gene tree for different alleles of Caja-G genes = HLA genes of Callithrix jacchus and the sequence K1_120D3-CajaG_45.

The tree is based on numbers of nucleotide substitutions per site, constructed by the neighbor-joining $(\mathrm{NJ})$ method. Numbers without decimals represent the percentage of bootstrap samples supporting the node using the maximum-parsimony method.

Caja-G4 is closest to HLA-G suggesting the greatest structural similarity between these two orthologoues genes. A major cluster is separated from HLA-F, a HLApseudogene having evolved in early HLA gene evolution. (Figure 5.5.1.2 and also 5.6.2.3). Upon alignment of DNA sequences, HLA genes show very high levels of identity with Caja-G. Therefore, the choice of Caja-G plausibly provided a probe hybridizing all HLA genes.

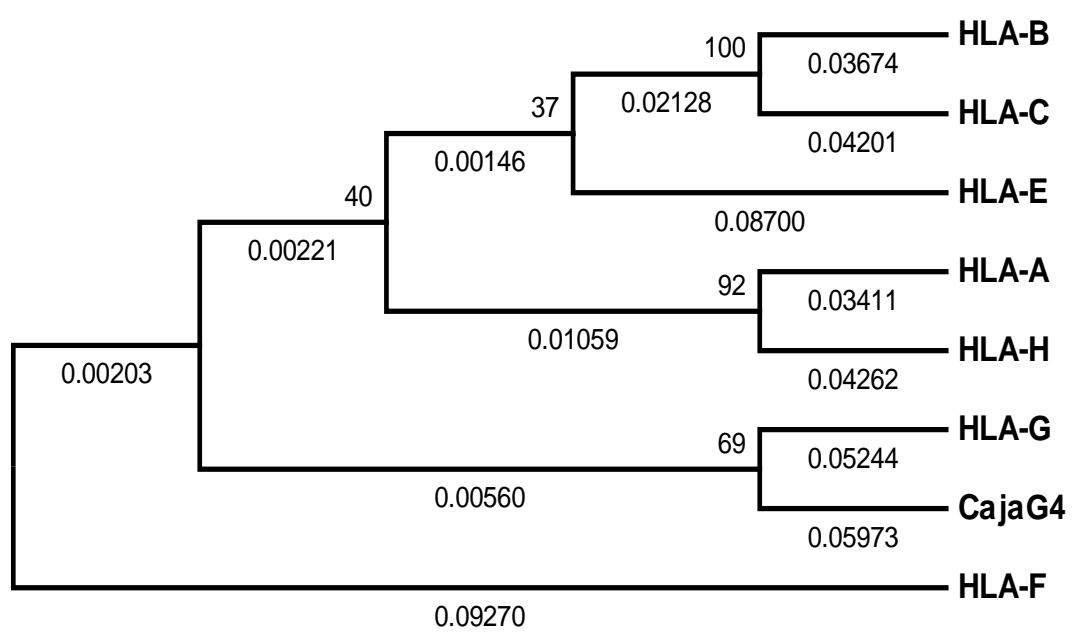

Figure 5.5.1.2: Gene tree for HLA genes and Caja-G4 of Callithrix jacchus.

The tree is based on numbers of nucleotide substitutions per site, constructed by the neighbor-joining (NJ) method. Numbers without decimals represent the percentage of bootstrap samples supporting the node using the maximum-parsimony method. 


\subsubsection{Gene trees of framework genes}

The topology of this tree of an orthologoues gene, essential and, therefore, very much conserved, shows the same evolutionary branching as seen for the following framework gene TCF19.

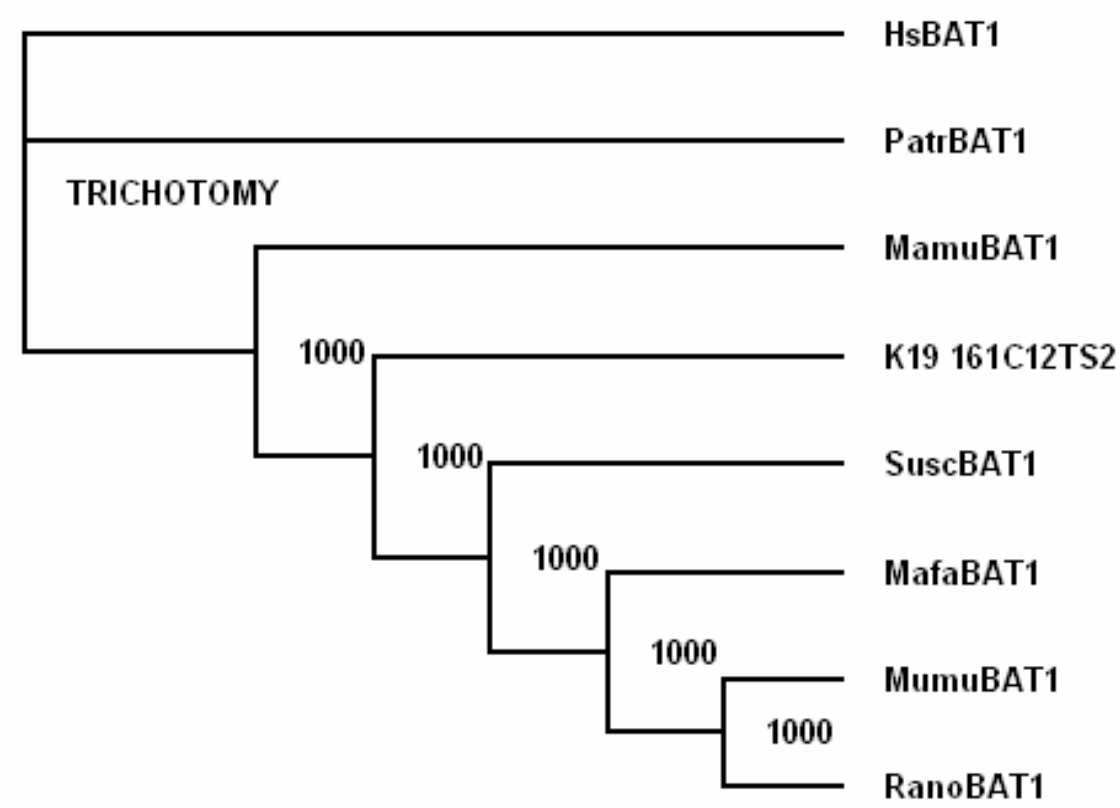

Figure 5.5.2.1: Phylogenetic tree of the framework gene BAT1 of the sequence K19 161C12-TS2 and other mammals.

Bota: Bos Taurus, Mumu: Musculus musculus, Rano: Rattus norvergicus, Patr: Pan troglodytes and Hs: Homo spiens. The tree is based on numbers of nucleotide substitutions per site, constructed by the neighbor-joining (NJ) method. Numbers without decimals represent the percentage of bootstrap samples supporting the node using the maximum-parsimony method.

Figure 5.5.2.2 contains the sequence K28-347D1TCF19-10 and the sequence of TC19 gene in other species. The tree shows that between this species have coexisted long enough for concerted evolution. 


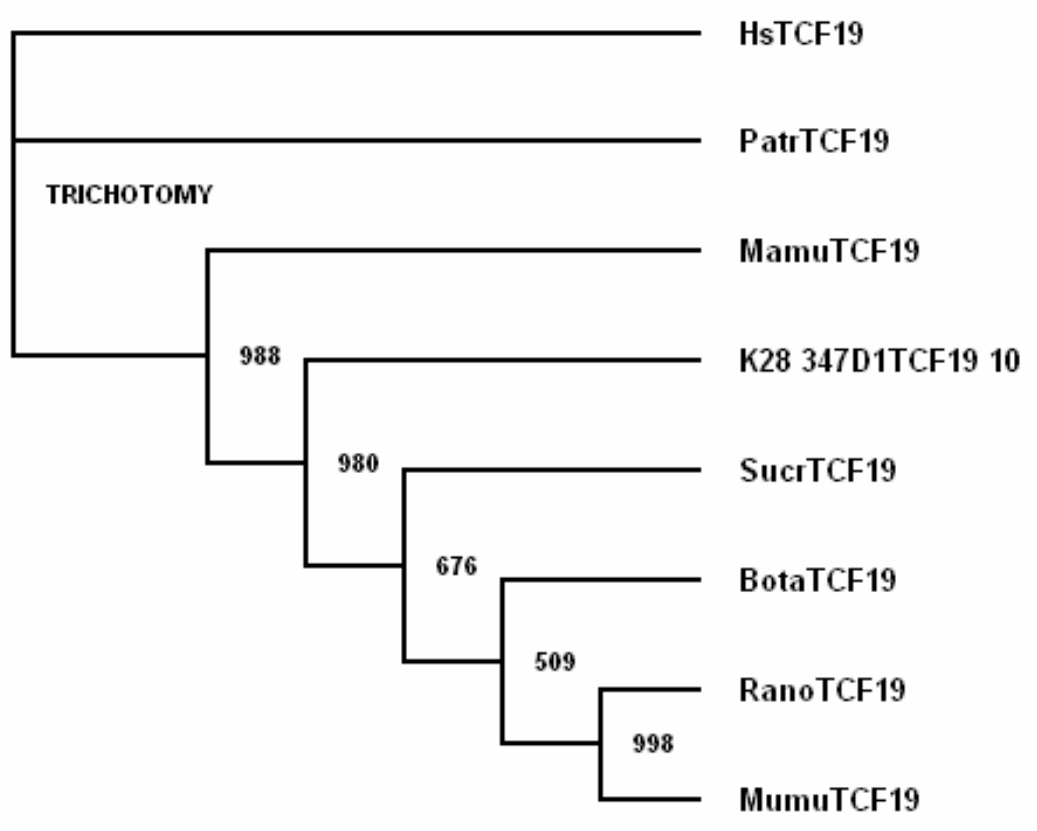

Figure 5.5.2.2: Phylogenetic tree of the framework genes TCF19 of the sequence K28347D1TCF19-10 and other mammals.

Note this tree was constructed with amino acidic sequences. Sucr: Sus scrofa, Mamu: Macaca mulatta, Patr: Pan troglodytes, Hs: Homo spiens, Mumu: Musculus musculus, Rano: Rattus norvergicus and Bota: Bos Taurus. The tree is based on numbers of nucleotide substitutions per site, constructed by the neighbor-joining (NJ) method. Numbers without decimals represent the percentage of bootstrap samples supporting the node using the maximum-parsimony method.

It seems easy to obtain with the programs used a branching order of trees for the evolution of genes and their (partial) DNA sequences. The phylogenetic results shown here seem to be in agreement with the overall phylogeny of the species considered - exept, however, that rodents Mumu and Rano are closer to primates than Bota and Sucr. The general phylogenetic order like needs not to be repeted in the evolution of every individual gene: a justification for doing trees. 


\subsection{TS1: teachings and challenges}

Dr. Takashi Shiina, when visiting our lab in autumn of 2004, had chosen from my selection of BAC clones the clone $\mathrm{K} 19-161 \mathrm{C} 12$ for a pilot sequencing in the MHC of Callithrix jacchus. In January 2005 he sent already two sequences, approx. 3000 bp each, to Dr. Lutz Walter, my supervisor at the time. He gave those to me. Later we called them TS1 and TS2 so we could distinguish them from the 53 sequences that I had done by myself.

Whereas TS2 was assigned to a fragment of BAT1, TS1 turned out to be a quite unusual sequence containing within 2994 bp for four different pseudogenes. These are not expressed any more, as we think, but may be of value for the study of evolution, i.e. of clustering by duplication, of regulatory sequences like promotor regions, and of occasional transposon insertion, and of a consensus sequences possibly important for DNA replication.

\subsubsection{A gene map for TS1, and for homologous genomic vicinities in the duplicons $B$ and $\mathrm{C}$ of $\mathrm{Hs}$}

To study the load of information contained in TS1 was not easy. Written in both directions and containing a ferritin pseudogene probably never seen in any MHC, it had to be taken apart into its natural parts that are arranged as shown in the gene map worked out by alignment with the two homologous regions in Hs DNA, one near HLA-B, the other near HLA-C, i.e. within the duplicons $B$ and $C$, respectively, see Figure 5.6.1. Clustal $X$ aligned the two class I pseudogenes called TS1-ps1 and -ps2 at very high identity. But furtheron through to the end of the sequence, it showed nothing but random base pairings, likewise in all primates and other mammals' DNA tested. Clustal $X$ would just not recognize the fact that behind a non-aligning insertion of more than 950 bp was located an at least formal continuation of ps2, a highly identical TS1 sequence called $v \mathrm{r}-\mathrm{c}$, that on this strand is complementary to the socalled "Vorspann" v located next to the 3' end of the pseudo-ferritin gene(psth1) on the other side. In aligning, Clustal $X$ does not gap such a long stretch as it routinely does for shorter distances in order to achieve maximum alignment.

The enigma of the upper part was solved, formally at least, by means of the AUGUSTUS program that predicts (parts of) genes by looking for criteria like start and stop signals for translation. It gave a first hint to a Hs ferritin heavy chain gene that upon direct questioning the Hs FTH1 (of the 11th chromosome) did very well 
align with TS1 entered "reverse-complementary" thus detecting a ferritin pseudogene psfth1 of Callithrix jacchus, see Figure 5.6.3.1.

For comparison with TS1, its homologous genomic vicinities in the Hs duplicons B and $C$ are defined in the comparative map, figure 5.6.1. Data are presented according to the class I region map established by $\mathrm{T}$. Shiina and his coworkers (2001).

In conclusion of these experiences: For study of alignment it has proven very useful to take sequences apart, possibly into natural subunits. These were aligned separately as well as joined again like ps1+ps2+v r-c for entry as a query. TS1 thus deprived of its ferritine gene insertion aligns totally, including $\vee \mathrm{r}-\mathrm{c}$, at more or less high identities with homologous DNAs of almost all primates and other mammals tested. No one besides Callithrix jacchus seemed to have a homologue of TS1psfth1.

Note: We must distinguish "reverse-complementary" as a term defining the direction for reading by computer programs. E.g. TS1 must be entered r-c for alignment of ferritin genes, see figure 5.6.3. This commands reading the left hand strand (complementary for the program) reversely. However, genes located on this strand, whereas possible read in opposite direction to those on the right hand strand (read forward in the sense of the computer), are not given a " $r$-c" as illustrated in figure 5.6.1.1. They may be assigned a $r-c$, however, as read in alignment of their antisense reversely like $v$ in ps1+ps2+v r-c. Please consider figure 5.6.1.1 and in particular the discussion on possible senses of $v$ and of $v r$-c. In duplicon $C$, the $v r-c$ homologue seems to be known as the pseudogene HCGIV-02. This unusual but conceivable double-sense, a rather different complementary-palindromic sense on the two complementary DNA strands, is making, of course, $v$ a difficult example. This sequence $\mathrm{v} r$-c seems even more remarkable as, moreover, a part of it occurs in various locations like in a 3'-terminal part of TS1-ps1 (Figure 5.6.2.5); it might be a matrix-association-region (MAR) or rather some other essential sequence as it is conserved even in a ancient pseudogene that other wise is very much altered by mutations (see chapter 5.6.5).

Another controversy must be understood: The Shiina scale base pair numbering for the class I region of the $\mathrm{MHC}$ in $\mathrm{Hs}$ and other primates runs towards telomere, the Venter genome bp numbering, however, in the opposite direction, throughout the 
whole chromosome. Both scales are used to identify all 55 sequences in table 5.4.1.1.

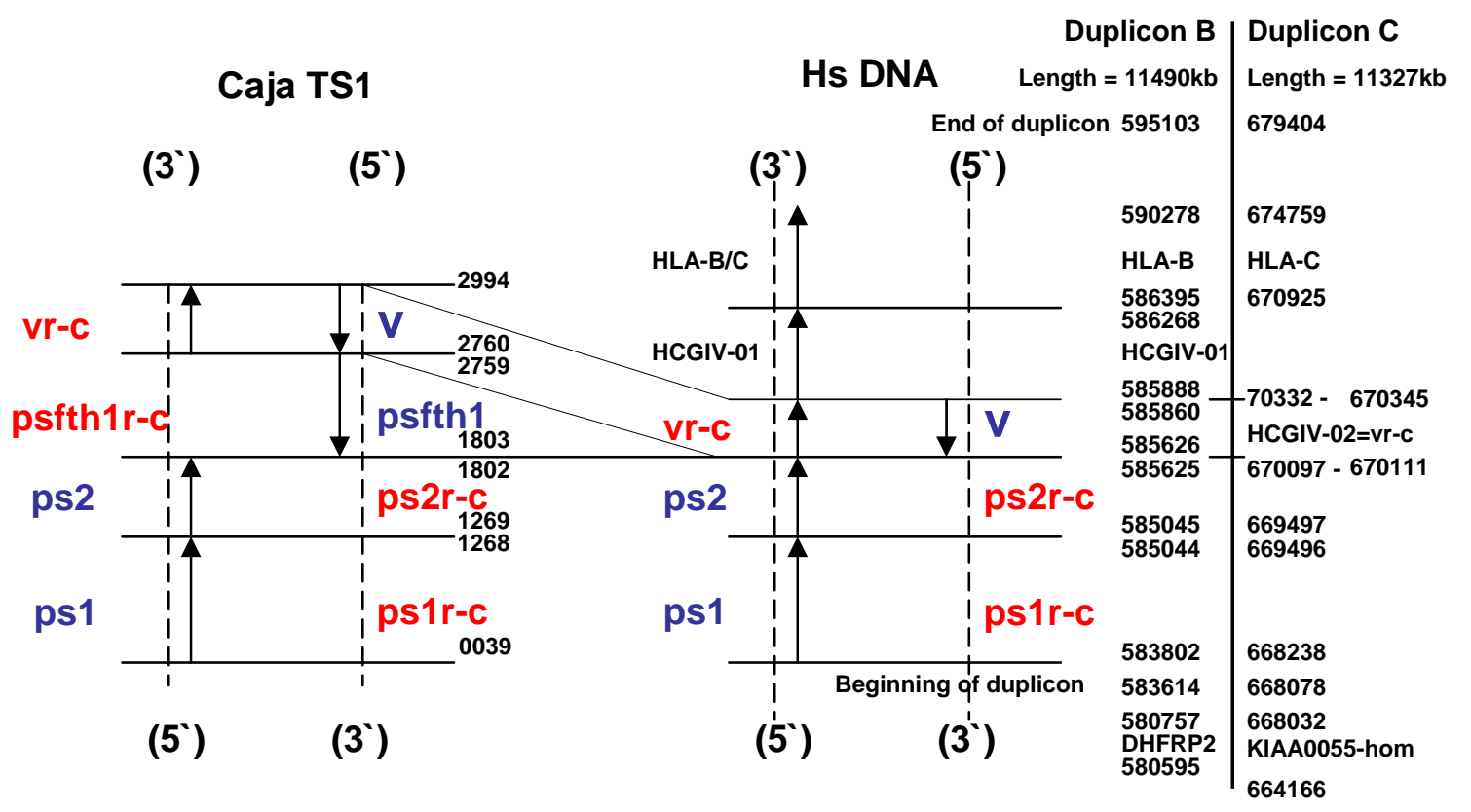

Figure 5.6.1.1: Gene maps for comparison of Caja TS1 with its homologous Hs DNA stretches showing the extension of the two highly identical duplicons $B$ and $C$ in the HLA-B/C section of the class I interval BAT1 - POU5F1. The insertion of a ferritin heavy chain pseudogene (pstth1) in TS1 - as marked by arrows - is reading on the left hand strand with decreasing Shiina bp numbers, i.e. opposite to the new class I pseudogenes TS1-ps1 and TS1-ps2. Therefore, psfth1 runs towards centromere. $v$ (for Vorspann) is a preliminary lab name. $v$ could be a sort of (pseudo)5'FR next to which the transposon psfth1 might have been inserted in Callithrix (or a near ancestor). Alternatively, v r-c could be just the 5' terminal part of a longer ps2 dissected for the insertion. In any case, the Caja sequence $v$ read reverse complementarily ( $\mathrm{r}-\mathrm{c})$, forward on the right and strand, is almost completely coincident and of very high identity with the known Hs pseudogene HCGIV-02 (figure 5.6.4.1), located ub duplicon C, from which TS1 seems to have been copied according to a higher identity in single that is considered to be part of duplicon C. a5'terminal part of $\mathrm{v} r$-c aligns at high identity in ps1 and other locations in and outside class I, thus suspicion of an essential consensus sequence (see Figure 5.6.2.5 and chapter 5.6.5). 


\subsubsection{The two new class I pseudogenes present in the HLA-B and -C duplicons} of Hs, likewise - not shown - of and Patr, other mammals Mamu, i.e. its 14 B

\section{duplicons}

668190-671500Shiina 1 583700-587000Shiina 1 K19_161C12TS1

Clustal Consensus

668190-671500Shiina 38 583700-587000Shiina 91 K19_161C12TS1 27

Clustal Consensus

668190-671500Shiina 127 583700-587000Shiina 181 K19 161C12TS1

Clustal Consensus

668190-671500Shiina 583700-587000Shiina K19_161C12TS1

Clustal Consensus

668190-671500Shiina 307 583700-587000Shiina 356 K19_161C12TS1

Clustal Consensus

668190-671500Shiina 583700-587000Shiina K19 161C12TS1

Clustal Consensus

668190-671500Shiina 487 583700-587000Shiina 536 K19_161C12TS1

Clustal Consensus

668190-671500Shiina 583700-587000Shiina K19 161C12TS1

Clustal Consensus

668190-671500Shiina 583700-587000Shiina K19_161C12TS1

Clustal Consensus

668190-671500Shiina 757 583700-587000Shiina 806 K19 161C12TS1

Clustal Consensus

668190-671500Shiina 583700-587000Shiina K19_161C12TS1 Clustal Consensus

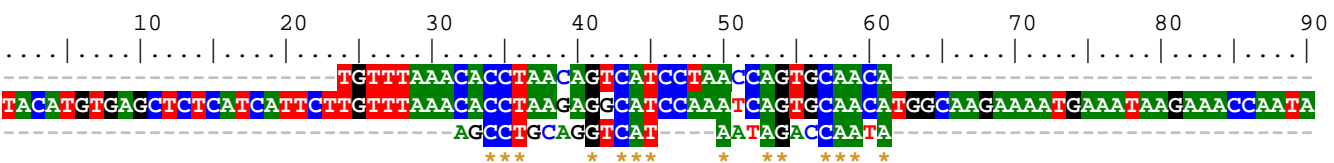

$\begin{array}{lllllllll}100 & 110 & 120 & 130 & 140 & 150 & 160 & 170 & 180\end{array}$ $\ldots|\ldots| \ldots|\ldots| \ldots|\ldots| \ldots|\ldots| \ldots|\ldots| \ldots|\ldots| \ldots|\ldots| \ldots|\ldots| \ldots|\ldots| \ldots|\ldots| \ldots \mid$ GAAGGACCAGGCATGGTGGCTCATGCCTGTAATCCCTGCATTTTGGGAGGTGAGGTGGAAGGATCACTTGAGTTCAGGAGTTTGAGACD GAAGGAGTGGGCATCGTGGTTCACACTTGTAATCCCTACATTTTGGGAAGCTGAGGTGGGAGGATCATTTCAGTTCAGGAGTTTGAGACC

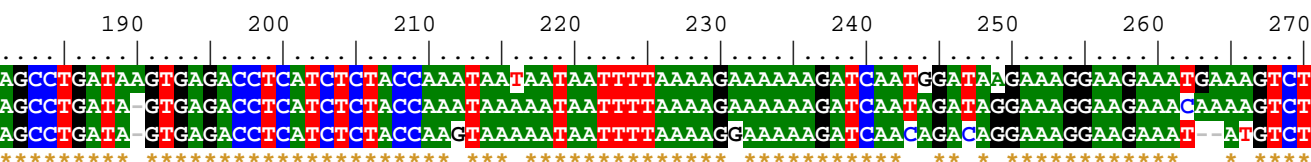
\begin{tabular}{|l|l|l|l|l|l|l|l}
280 & 290 & 300 & 310 & 320 & 330 & 340 & 350
\end{tabular} $\ldots|\ldots| \ldots|\ldots| \ldots|\ldots| \ldots|\ldots| \ldots|\ldots| \ldots|\ldots| \ldots|\ldots| \ldots|\ldots| \ldots|\ldots| \ldots|\ldots| \ldots|\ldots|$ TTCTTTGTCACCAGCTTCATTG TT - - GTCACCAGCTTCATTGCACATGCAGAAAACACTAGGAAATTCTGAAAAAGTCTCTGGAATTAATTATTGAATTTACAAA-TAGT

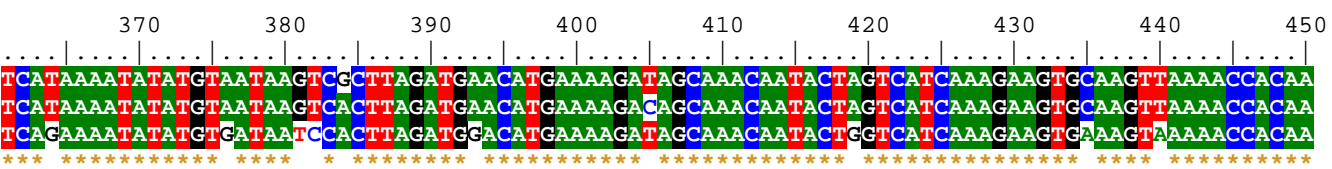

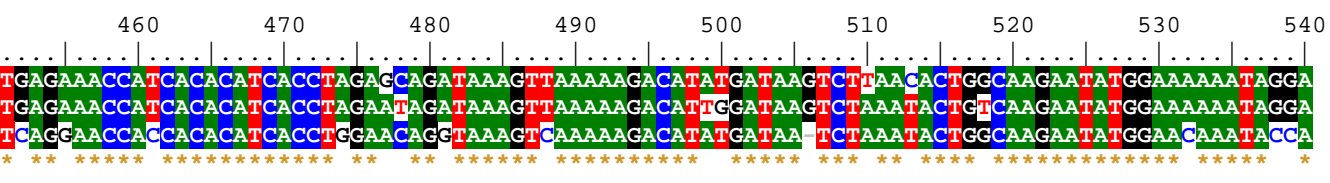

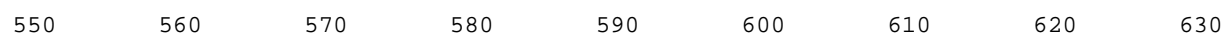

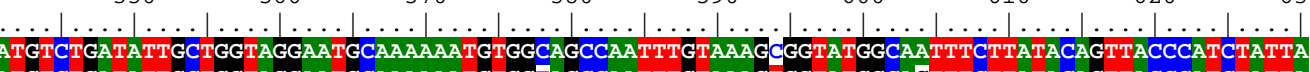
ATGTCTGATATTGCTGGTAGGAATGCAAAAAATGTGGTAGCCAATTTGTAAAGTGGTATGGCAGTTTCTTATACAGTTACCCATCTATTA ATGTCTG-TATTGCTGGTTTGAATGCAAAAAATGTTGCAGCCAATTTGTAAAGTGGTTUAGCAATTTCTTATACAGCTGCCCATCCACTA

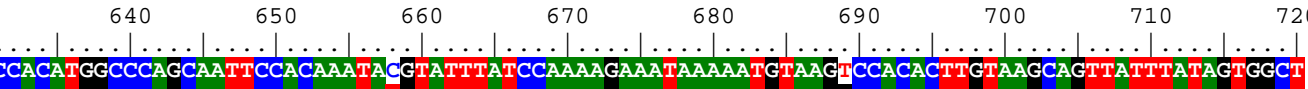 CCACATGGCCCAGCAATTCCACAAATATGTATTTATCCAAAAGAAATAAAAATGTAAGGCCACACTTGTAAGCAGTTATTTATAGTGGCT CCACAGGACCCAGGAATCCCACATATATGTATTTATCCAAAAGAAATAAAAGTGTAAGGCCACACTTGTAAGCAGTTACTTGTAGTGGCT

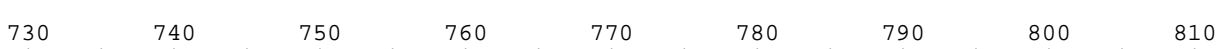
$\ldots|\ldots| \ldots|\ldots| \ldots|\ldots| \ldots|\ldots| \ldots|\ldots| \ldots|\ldots| \ldots|\ldots| \ldots|\ldots| \ldots|\ldots| \ldots|\ldots| \ldots|\ldots| \ldots \mid$ TCATTAATAACAAGCCCTAACTGGAGGAATCCACATGTCTATCAACTGGAGACACAGAAACCAATGAATAAACTGGGATTCCAGCAATAC TCATTAATAACACACCCTAACTGGAGTGATCCACATGTCTATCAACTGGAGA-ACAGAAACCGATGAGTAAACTGGGATTCCAGCAATAC

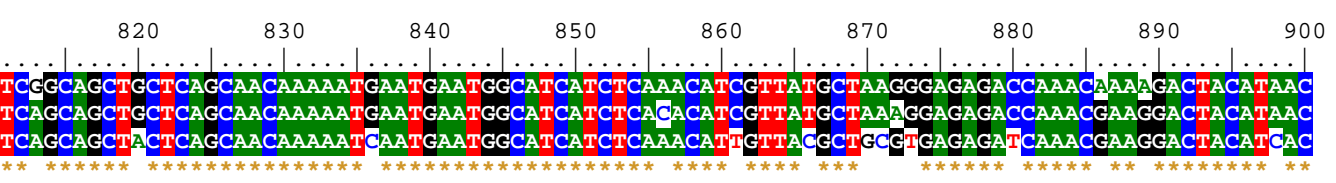

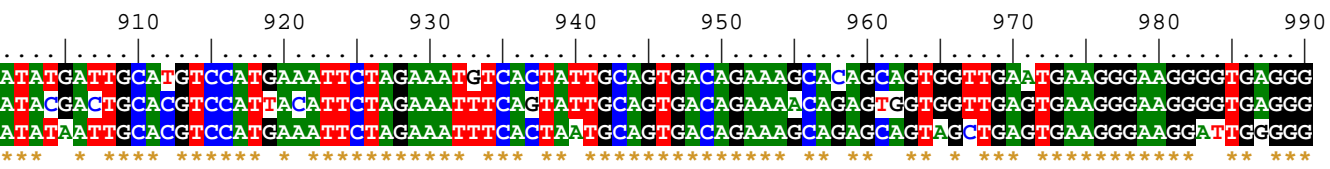


Alignment has been performed by Clustal $X$. The nucleotide sequence of $H$ s to duplicon B and C has ID: BA000025. TS1-ps1 is one of the rarer complete class I pseudogenes. It shows a remainder of its former start codon changed to ATC (the Hs ps1's homologues in both duplicons still have their ATG) and a poly A tail. TS1-ps2 seems to be a fragment pseudogene, possibly interrupted by the insertion of a pseudoferritin gene (psfth1) as shown in Figure 5.6.3.1.

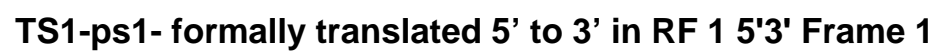

XXPAGHNRPIEGVGIVVHTCNPYILGS Stop G GRIISVQEFETSLIVRPHL Y Q VKIILKGKRSTDRKGR N Met SLSPASLH Met Q KTLGNSEKVSG I N Y Stop I Y K Stop F R K Y Met Stop Stop S T Stop Met D Met K R Stop Q T I L I K E V K V K T T I R N H H T SPGTGKVKKTYDNLNTGKN Met E Q I P Met SVLLV Stop Met Q K Met L Q P I C K V V Stop Q F L I Q L P I H Y HR T Q E S H I Y V F I Q K K Stop K C K A T L V S S Y L Stop W L H Stop Stop H T L T G V I H Met S I N W R T E T D E Stop T G I P A I L S S Y S A T K I N E W H H L K H C Y A A Stop E I K R R T T S H I Met H V HE I L E I S L Met Q Stop Q K A E Q Stop L S E G K DW GWKAR I K Stop K G A Stop R K F L G R N C S L Q CHNSGVT G Q WE LRRKE GGEGQREGLGKQEVRRRSREKDSKAVKEPSRGFFACRVSLLTSVVVT RL Q IIS I C TKKR S Stop T W Stop I L L INA L S Q KKKK

\section{TS1-ps1- 5'3' Frame 2}

X S L Q V II D Q Stop K E W A S W F T L VI P T F WE A E V G G S F Q F R S LR P A Stop Stop Stop D L I S T K Stop K Stop F Stop K E K D Q Q T G K E E I C L C H Q L H C T C R K H Stop E I LKKSLELIIEFTNSSEN ICDNPLRW T Stop KD SKQYW S S K K Stop K Stop K P Q SGTTTHHLEQVKSKRH Met II Stop I LAR I W NKYQCLYCWFECKKC C S F V KWFSNFLYSCPSTTTGPRNPTYMet YLSKRNKSVRPHL Stop A VT C S G F I N N T P Stop L E Stop S T C L S T GE Q K P Met SK L G F Q Q Y S A A T Q Q Q K S Met N G I I S N I V T LRER S N E G L H H I Stop C T S Met K F Stop K F H Stop C S D R K Q S S S Stop V K GRIG G GKLELNRKGHRESF Stop GKETVLYS V T I L E Stop L G S G N Stop GER REER GREKVW G SRR Stop GE G A GKRTLKQ Stop R S L A G G S L H A E F L Y Stop P V W Stop L H D Y K Stop Y Q Y A P K S A A K P G K F Y Y A Stop Met P Stop A K K K K K

\section{TS1-ps1- 5'3' Frame 3}

X A C R S Stop Stop T N R R S G HR G S H L Stop S L H F G K L R W E D H F S S G V Stop D Q PDSETSSLPSKNNFKRKKINRQERKKYVFVTSFIAHAENTRKF StopKSL W N Stop L L N L Q IV Q K I Y VIIHLDGHEKI ANNTGHQRSESKNHNQEPPH IT W N R Stop S Q K D I Stop Stop S K Y W Q E Y G T N T N V C I A G L N A K N V A A N L Stop S G LA ISYTA A H P L P Q DPGIPHICIYPKEIKV Stop GHTCKQLLVVASLITHPN W S D P H Y Q LENRNR Stop VNWDS SNTQ Q L L SNKN Q Stop Met A S S Q T LR

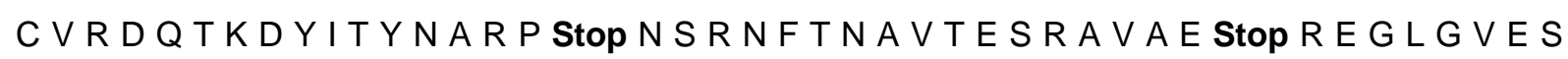
Stop N Stop I ER G I E K VFREKKLFSTVS Q FW S DW A VGTKEKG GRR G A R R 
S G E A G G K E K E Q G K G L Stop S S E G A Stop Q G V L C Met Q S F S T D Q C G S Y T T T NNIN Met HQKA Q L LVNFI Met HKCPKPKKKKK

Figure 5.6.2.2: Formal amino acid (aa) translations for TS1-ps1 as given by Swiss-prot in three reading frames (RF) from the (mRNA) 5'-end.

RF I is starting at third, RF II at first, RF III at second base of the DNA sequence (shown in the Annex). In each of these RF, many mutations have spoiled a potential original sense, as recognised by many Stop and Met codons ruining potential coding for polypeptides of any useful (and meaningful) length. Moreover, chances for transcription and translation are destroied by mutation of signals required for initiation of transcription in the 5'FR (TATA box, CAG and other signals). Consequent nontranslatability by itself has removed natural selection pressure against deleterious mutations in these former genes, whereas general mechanisms for conservation of unchanged genetic information have kept such molecular fossils still good enough for investigation of the evolution of MHC class I molecules. Moreover, there may be still some essential sequence(s) that even DNA of pseudogenes has to maintain, as observed and discussed below (Figure 5.6.2.5 and chapter 5.6.5). 


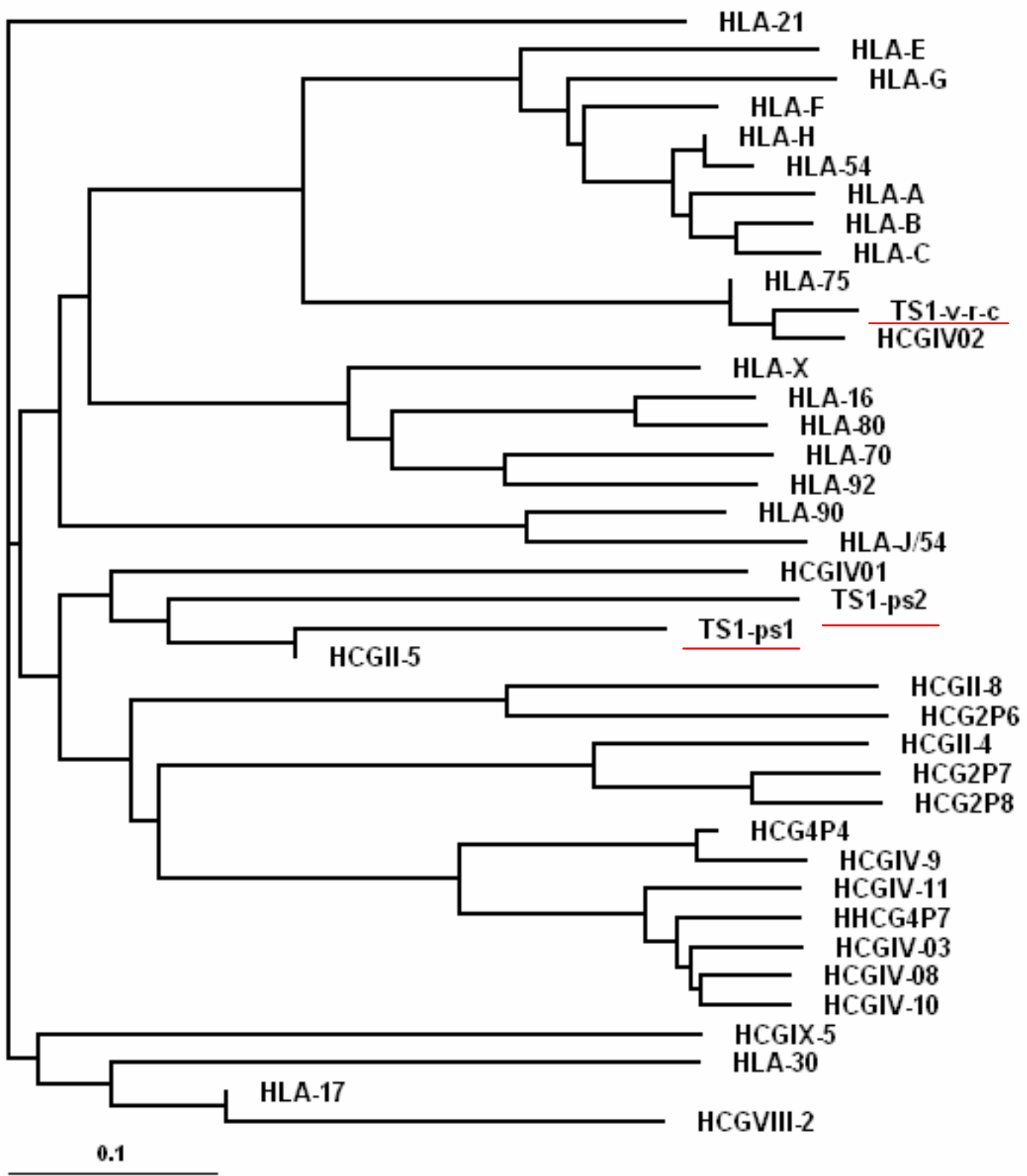

Figure 5.6.2.3: Evolutionary tree considering the classical and non-classical HLA genes and the class I pseudogenes of Hs giving a chance to the Caja TS1ps1, -ps2 and -v r-c for finding their place within the known clusters.

Con-clustering of TS1-v r-C together with HCGIV-02 is expected by their nearly identical sequences. Their showing up in the HLA class la and Ib-cluster, however, may be worth further study, in particular with respect of 5'FR. TS1-ps1 and -ps2 seem most related to HCGIV-01 related to the clusters II and IV. This result must be studied in more detail, looking carefully at alignment of different parts, segments of the sequences. It has been found that three thirds of TS1-ps1 receive different classifications when aligned separately and evaluated for the tree. The tree is based on numbers of nucleotide substitutions per site, constructed by the neighbor-joining 
(NJ) method. Numbers without decimals represent the percentage of bootstrap samples supporting the node using the maximum-parsimony method.

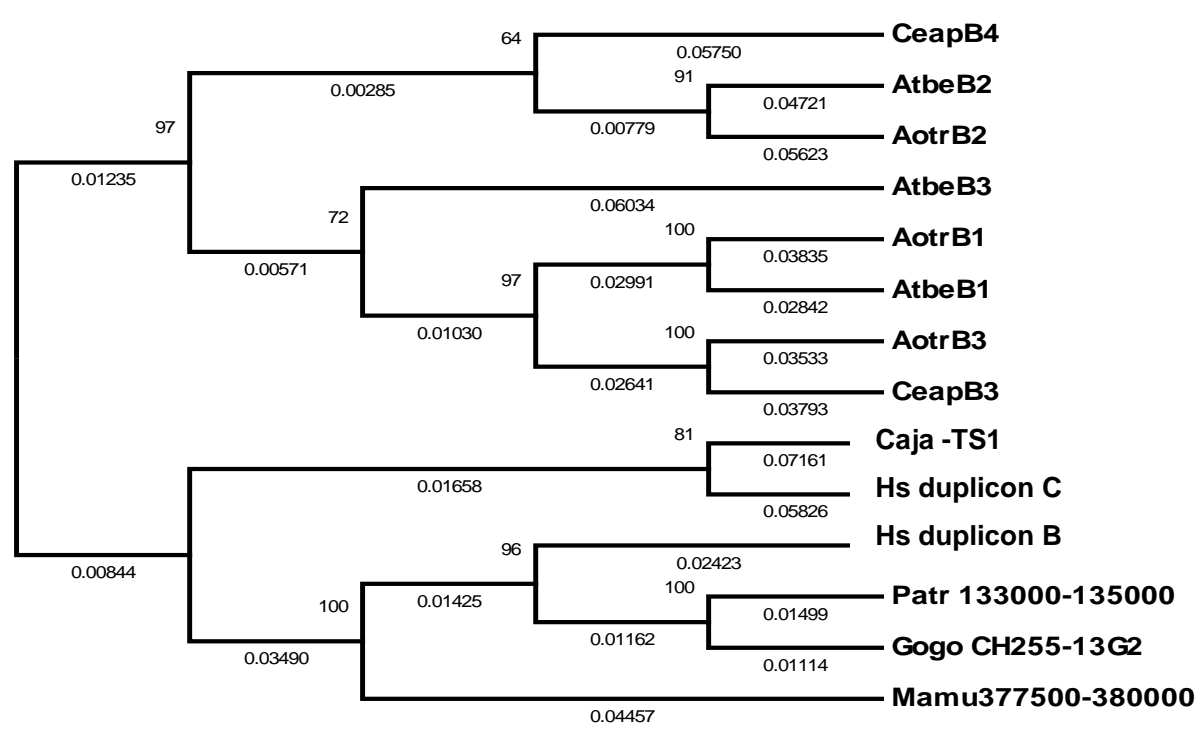

Figure 5.6.2.4: Phylogenetic tree of the sequence K19-161C12-TS1 and its homologues in Hs and other primates.

Note the tree was constructed with amino acidic sequences obtained formal translations by Swiss-prot. Atbe: Ateles belzebuth, Aotr: Aotus trivirgatus, Ceap: Cebus apella, Patr: Pan troglodytes, Gogo: Gorilla gorilla and Mamu: Macaca mulatta. The tree is based on numbers of nucleotide substitutions per site, constructed by the neighbor-joining (NJ) method. Numbers without decimals represent the percentage of bootstrap samples supporting the node using the maximum-parsimony method.

This preliminary study of relationships between TS1-ps1+ps2+v r-c homologous sequences in the $B$ duplicon of various primates by a phylogenetic tree confirms $B$ duplicon in all primates tested. Surprisingly, TS1 homologous Hs duplicon B DNA turns out to be nearest to a phylum of Patr, Gogo and Mamu (represented by for its B4 duplicon); Hs duplicon C DNA, however, is nearest to TS1-ps1+ps2+v r-C. This is a third piece of evidence obtained in favor of TS1 stemming from a Caja $C$ rather a than B duplicon. 


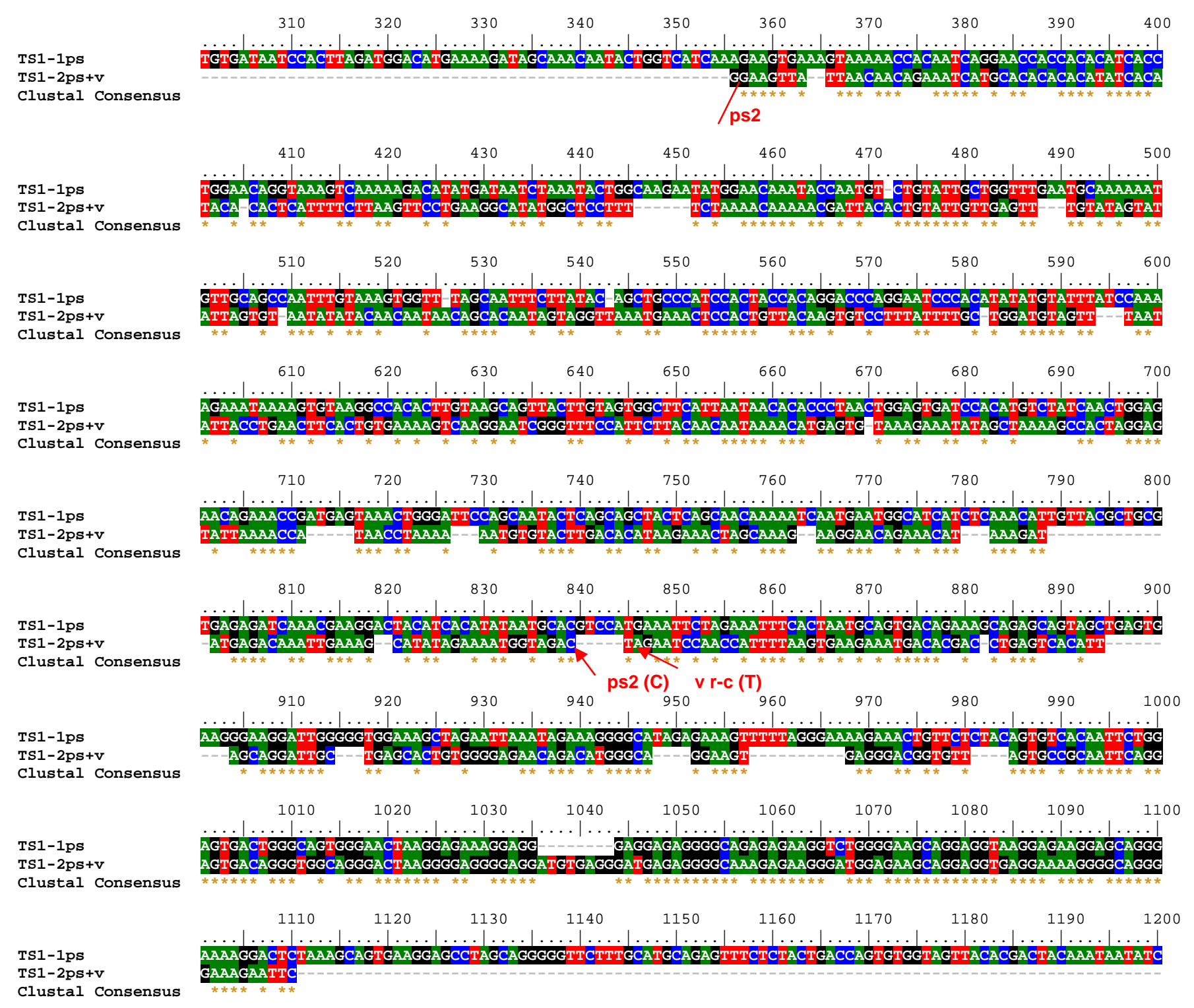

Figure 5.6.2.5: Observation of high identity alignment of the 5'-terminal part of TS1-v r-c with a section 5' terminal end, near the TS1-ps1.

Before this stretch, TS1-ps2 and a 3' - terminal part of $v \mathrm{r}-\mathrm{c}$, also aligned for comparison, show a rather normal random distribution of base identities. Alignment has been performed between TS1-p1, TS1-2ps+v by Clustal X.

TS1-ps1 and -ps2 were aligned also with each other. Their degree of identity might be slightly above expectation for a random distribution. It should be calculated for many ps1 and ps2 homologues apparent in class I duplicons of primates.

Quite safe, however, seemed a high identity between alignment of TS1-ps1 and TS1v r-c. A part of v r-c, bases 100 to 235 on the TS1 map, showed $59 \%$ identity with bases 985 to 1110 of TS1-ps1, i.e. near the 5' terminal end of ps1. This observation 
contrasts to the many mutations as seen e.g. by stop and Met codons in most parts of the formal translations of the no more expressed pseudogene (Figure 5.6.2.2).

\subsubsection{A ferritin heavy chain pseudogene in the MHC of Callithrix jacchus}

All living cells need apoferritin, its light and heavy chain protein subunits, 24 of which (at different ratios in different tissues) combine to form a package for storage of 4500 $\mathrm{Fe}^{2+}$ ions. Each subunit's tertiary structure contains $4 \alpha$-helices composed of many very conservative amino acids. Primates and other mammals usually have one expressed ferritin gene from which during evolution have descended altogether a few pseudo ferritin genes, six heavy chain pseudogenes in Hs (Constanzo et al., 1986).

We have taken a closer look at sequence and evolution of the pseudoferritin gene TS1-psfth1 found and localized as shown in the map, figure 5.6.1.1. TS1-psfth1 seems unique for the MHC as far as we can enquire in sequences known today. It was not found in Hs nor any other primate or mammal. For many animals, mRNAs have been sequenced only no complete DNA as is needed for further investigation.

We shall look how psfth1 aligns with the human FTH1 gene as seen in figure 5.6.3.1. Thereafter, we see both aligned together with all Hs heavy chain pseudogenes. A gene tree will suggest then a place in evolution for psfth1 (Figure 5.6.3.2). On the protein level, where mutated genes look more conserved compared than in their nucleotide sequence, we look back from $\mathrm{Hs}$ and Caja over a selection of animals down to Caenorhabditis. So we go far below the first animals having an MHC i.e. teleosts (Dare is included). If psfth1 would be such an old transposon we could not possibly trace it to such an old ancestor, because of no complete DNA sequences being available, also of insufficient knowledge on early immune proteins and $\mathrm{MHC}$ organizations.

Figure 5.6.3.2 shows a very high degree of identity particularly in the large middle part of the molecules most of which were coding for the $\alpha$-helical parts of ferritin once, i.e. in the still expressed evolutionary state of the ferritin gene at the time of their origin. Note, only the expressed gene $\mathrm{H} 11=\mathrm{FTH} 1$ has a TATAA box, the CAG signal for transcription start, and alike some of the pseudogenes and TS1-psfth1, the 28 nucleotide signal in the 5'FR before the translation start, ATG. The 28 nucleotide signal is nearly identical in Hs, rat and several other mammals including Caja (Murray et al., 1987). 
Hs FTH1

K19_161C12TS1rev-comp 1

Clustal Consensus

Hs FTH1

K19_161C12TS1rev-comp 1 Clustal Consensus

Hs_FTH1 K19 161C12TS1rev-comp 181 Clustal Consensus 1

Hs FTH1 Clustal Consensus

Hs_FTH1

(110

Hs_FTH1

K19 161C12TS1rev-comp 224

Clustal Consensus 190

Hs_FTH1

19 161C12TS1rev-comp 540

Clustal Consensus

Hs_FTH1

K19 161C12TS1rev-comp 630

Clustal Consensus

Hs_FTH1

K19_161C12TS1rev-comp

Clustal Consensus

Hs FTH1

K19_161C12TS1rev-comp 810

Clustal Consensus

Hs_FTH1

K19_161C12TS1rev-comp

Clustal Consensus

Hs_FTH1

K19 161C12TS1rev-comp

Clustal Consensus

Hs_FTH1

K19_161C12TS1rev-comp clustal Consensus

Hs FTH1

Clustal Consensus 526

674 900 612 764 694 71

$\begin{array}{ccccccccc}10 & 20 & 30 & 40 & 50 & 60 & 70 & 80 & 90\end{array}$

GAATTCTTTCCCCTGCCCCTTTTCCTCACCTCCTGCTTCTCCATCCCTTCTCTTTGCCCCTCTCATCCCTCACATCCTCCCCTCCCCTTA

TS1-v

$\begin{array}{lllllllll}100 & 110 & 120 & 130 & 140 & 150 & 160 & 170 & 180\end{array}$ $\ldots|\ldots| \ldots|\ldots| \ldots|\ldots| \ldots|\ldots| \ldots|\ldots| \ldots|\ldots| \ldots|\ldots| \ldots|\ldots| \ldots|\ldots| \ldots|\ldots| \ldots|\ldots| \ldots \mid$

GTCCCTGCCACCCTGTCACTCCT GAATTGCGGCACTAACACCGTCCCTCACTTCCTGCCCATGTCTGTTCTCCCCACAGTGCTCAGCAAT

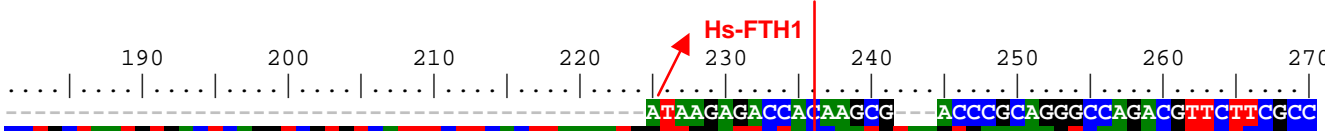
CCTGCTAATG GACTCAGGTCGTGTCATTTCTTCACTTAAAATGGTTGGATTCTAC:CAATGTAAGTGAAAAAGTCAAGACGTTCTCCGCC

$$
\stackrel{* * *}{\longrightarrow} \stackrel{*}{*} \text { TS1-fth1 }
$$

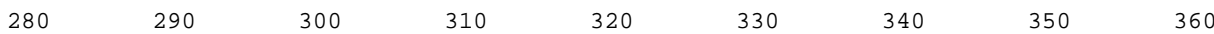

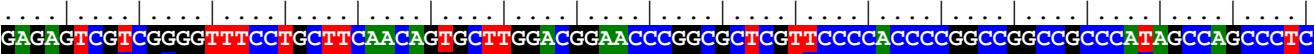
GAGAGTCGCCGCGGTTTCCTGCTTCAACAGGGCTTGGACGGAACCCGGCGCTCGTCCCCCACCCCGGCCGGCCGCCCACAGCCAGCCCTC

$\begin{array}{lllllllll}370 & 380 & 390 & 400 & 410 & 420 & 430 & 440 & 450\end{array}$ $\ldots \ldots|\ldots| \ldots|\ldots| \ldots|\ldots| \ldots|\ldots| \ldots|\ldots| \ldots|\ldots| \ldots|\ldots| \ldots|\ldots| \ldots|\ldots| \ldots|\ldots| \ldots \mid$ CCTCACCTCCACACCGCGCCCTCCGACCTCTCCAAGGCCCC-GCCGCCTCTCCAGCGCCGCGCAGCCACCGCCGCCGCCGCCGCCTCTCC

$\begin{array}{lllllllll}460 & 470 & 480 & 490 & 500 & 510 & 520 & 530 & 540\end{array}$

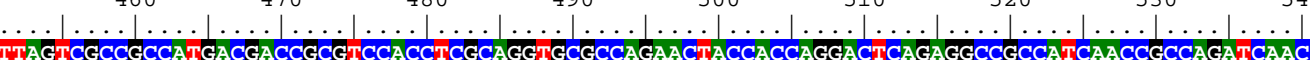
TTAGCAGCCGCGGTGACGACCGCATCACCTTCGCAGGTGCGCCAGAACTACCACCAGGATTCAGAGGCCGCCATCAACCGCCAGATCAAC $\begin{array}{llllllll}550 & 560 & 570 & 580 & 590 & 600 & 610 & 620\end{array}$ $\ldots|\ldots| \ldots|\ldots| \ldots|\ldots| \ldots|\ldots| \ldots|\ldots| \ldots|\ldots| \ldots|\ldots| \ldots|\ldots| \ldots|\ldots| \ldots|\ldots| \ldots|\ldots|$ CCGGAGCTCTACGCCTCCTATGTTTACCTGTCCATGTCTTACTACTTTGACCGTGATGACGTGGCTTTGAAGAACTTTGCCAAGTACTTT

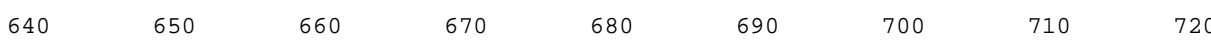
$\ldots|\ldots| \ldots|\ldots| \ldots|\ldots| \ldots|\ldots| \ldots|\ldots| \ldots|\ldots| \ldots|\ldots| \ldots|\ldots| \ldots|\ldots| \ldots|\ldots| \ldots \mid$ CTTCACCAATCTCATGAGGAGAGGGAACATGCCGAGAAACTGATGAAGCTGCAGAACCAACGAGGTGGCCTCATCTTCCTTCAGGATGTC

$\begin{array}{lllllllll}730 & 740 & 750 & 760 & 770 & 780 & 790 & 800 & 810\end{array}$ $\ldots|\ldots| \ldots|\ldots| \ldots|\ldots| \ldots|\ldots| \ldots|\ldots| \ldots|\ldots| \ldots|\ldots| \ldots|\ldots| \ldots|\ldots| \ldots|\ldots| \ldots \mid$ AAGAAACCAGACCATGATGACTGAAAGAGCGGGCTGAATGCGATGGAGTGTGCATTACATTTGGAAAAAGATGTGAATCAGTCACTACTG

$\begin{array}{lllllllll}820 & 830 & 840 & 850 & 860 & 870 & 880 & 890 & 900\end{array}$ GAACTGCACAAACTGGCACTGACAAAAATGACCCCATTTGTGTGACTTCATTGAGACACATTACCTGAATGAGCAGGTGAAGCCATC GAACTGCACAAACT GGCCACTGACAAAAATGACCCCCATTTGTGAAACTTCATTGAGACACATTACCTGAATAAGCAGGTGAAATCCATC

$\begin{array}{lllllllll}910 & 920 & 930 & 940 & 950 & 960 & 970 & 980 & 990\end{array}$ AAAGAATTGGGTGACCACGTGACCAACTTGCGCAAGATGGGAGCGCCGAATCTGGCTTGGCGGATATCTCTTTGACAAGCACACCCTG AAAGAATTGGGTGGCCACGTGACCAACTTGCTCAAGTTGGGAGCAACTGAATCTGGCTTGGAAGAATATCTCTTTGACAAGCACACCCTG

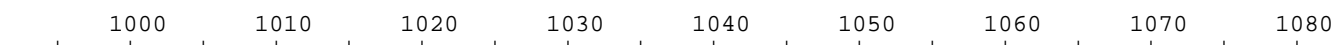

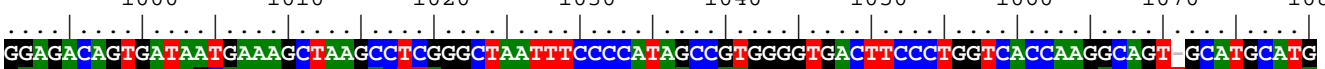
GAAGACAGT GATAGCAAAAGCTAAGACTTAGGCTAATTTCCCCATAACCAAGGGGTGACTTCCCTGGTCACCAAGACAGTTGCATGCATA

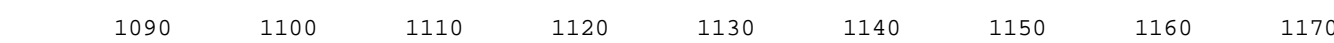
$\ldots|\ldots| \ldots|\ldots| \ldots|\ldots| \ldots|\ldots| \ldots|\ldots| \ldots|\ldots| \ldots|\ldots| \ldots|\ldots| \ldots|\ldots| \ldots|\ldots| \ldots|\ldots|$ TTGGGGTTCCCTTTACCCTTTCTATAAGTTGTACCAAAACATCCACTTAAGTTCTT

$1240 \quad 1250$

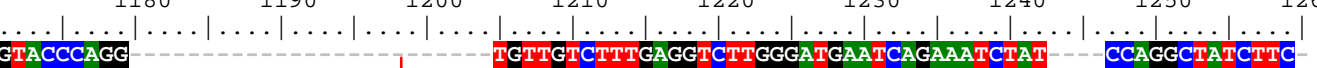
GTGCCAAAAAAAAAAAAAAAAAGAAAAGTCTACCATTTTCTATATGCTTTCAATTTGTCTCATATCTTTATGTTTCTGTTCCTTCTTTG

$$
\text { TS1-fth1 TS1-ps2 }
$$




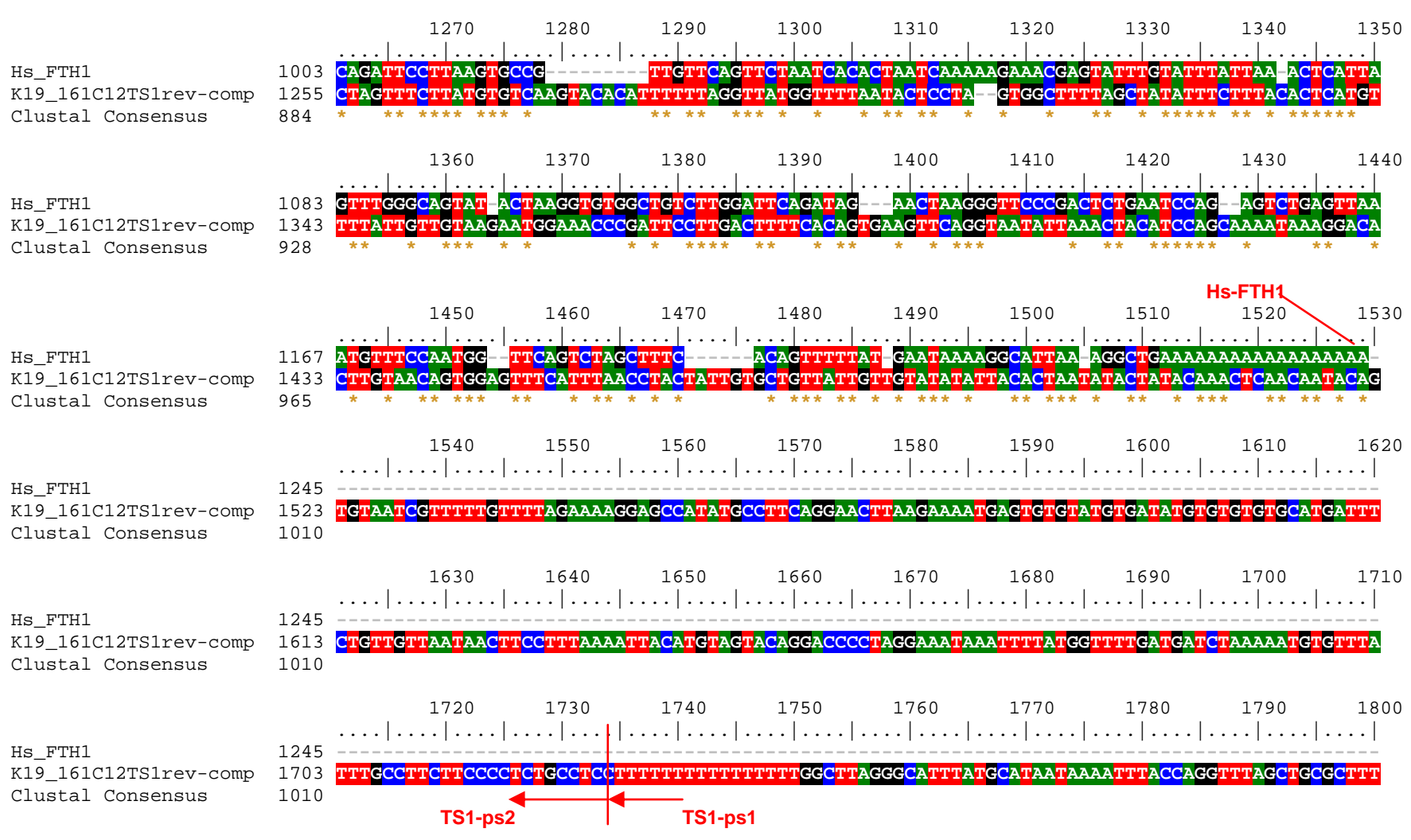

Figure 5.6.3.1: Alignment of TS1 psfth1 with the only expressed Hs ferritin gene FTH1 located on chromosome 11.

TS1 entered r-c, at its 3' end shows $v$ as known from figure 5.6.1.1. TS1-psfth1 aligns with FTH1, in their beginnings with \pm random identity, from bp 257 on this reverse scale, however, at very high identity up to shortly before beginning of as through to the poly A tail of TS1-psfth1. Thereafter, Hs FTH1 shoes only random identity, because DNA contains ps2 there (on its complementary strand). Alignment has been performed between K19_161C12TS1rev-comp and FTH1 gene of Hs (ID: NT033927) by Clustal $\mathrm{X}$. 
Hs3

Hs3 - 3

Hs2

Hs11

Hs8

TS1-psfth1

Hs 2-2

Clustal Consensus

Hs3

Hs3-3

Hs2

Hs13

Hs11

TS1-psfth1

Hs2-2

Clustal Consensus

Hs3

Hs 3 - 3

Hs2

Hs13

Hs11

Hs8

TS1-psfth1

Hs2 - 2

Clustal Consensus

Hs3

Hs3-3

Hs2

Hs13

Hs11

Hs8

TS1 - psfth1

Hs2-2

Clustal Consensus

Hs3

Hs3-3

Hs2

Hs13

Hs11

Hs8

TS1-psfth1

Hs2 - 2

Clustal Consensus

Hs3

Hs3-3

Hs2

Hs13

Hs11

Hs8

TS1-psfth1

Hs2-2

Clustal Consensus

Hs3

Hs3-3

Hs2

Hs13

Hs11

Hs8

TS1-psfth1

Hs2-2

Clustal Consensus

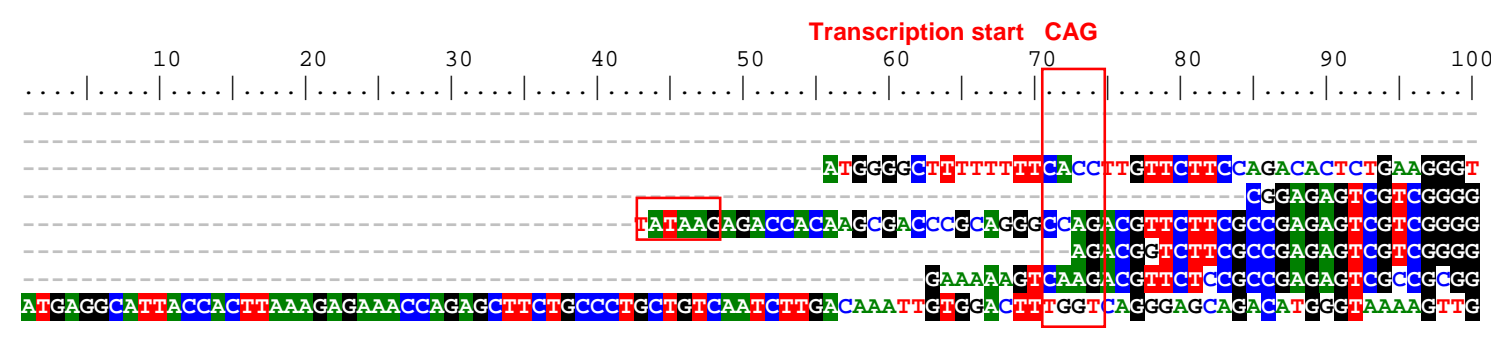

28 nucleotide signal

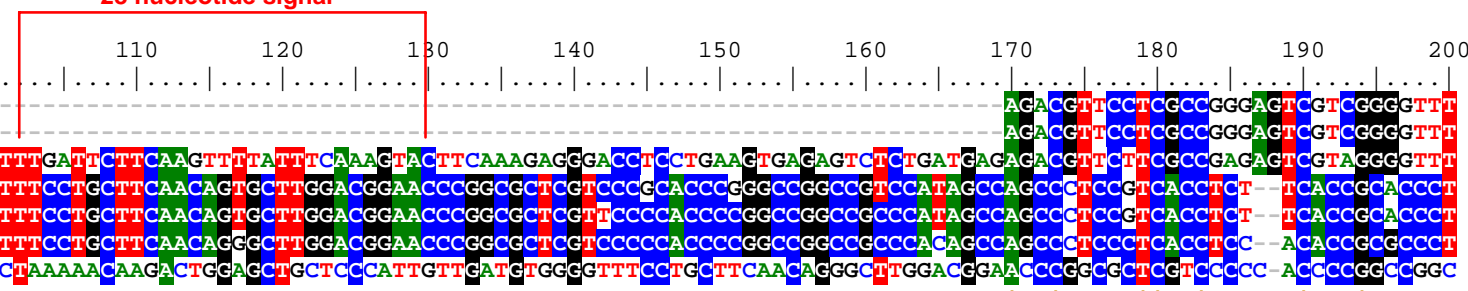

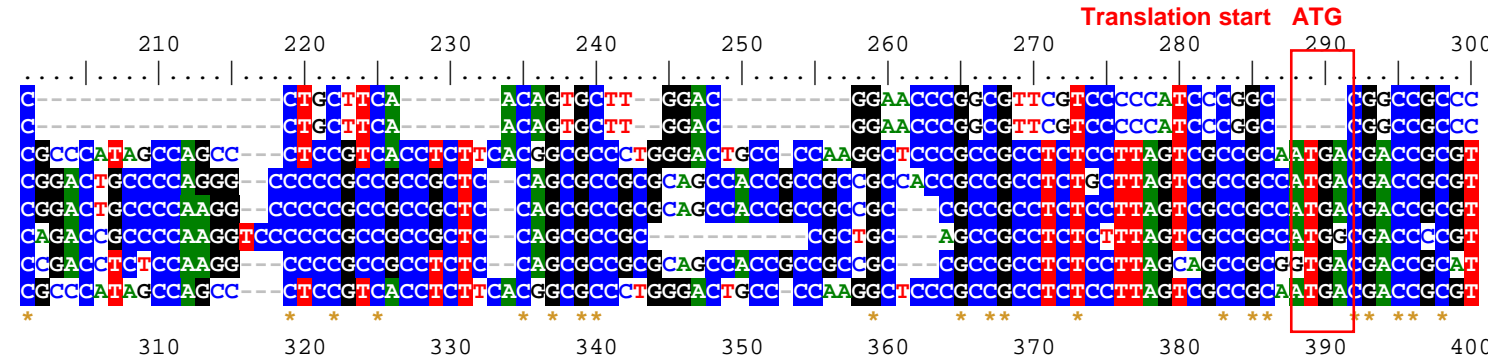

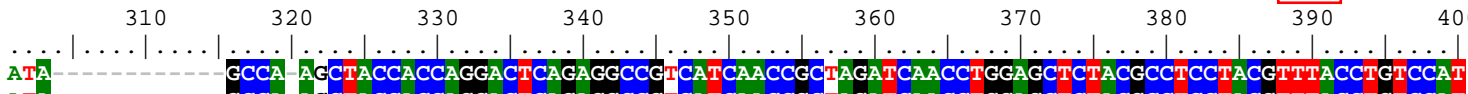
ATA - GCCA-AGCTACCACCAGGACTCAGAGGCCGTCATCAACCGCTAGATCAACCTGGAGCTCTACGCCTCCTACGTTTACCTGTCCAT CCACCTCGCAGGTGCGCAGAACTACCACCAGTACTCAGAGGCCCCATCAACCGCCAGATCAACCTGGAGCACTACGCCTCCTAAGTTTACCTGTCCC CCGCCTCGCAGG TGCGCCAGAACTCCCACCAGGACTCAGAGGCCGCCATAAACCGCCAGATCAACCTGGAGCTCTACGCCTCCTACGTTTACCTGTCCAT CCACCTCGCAGG TGCGCCAGAACTACCACCAGGACTCAGAGGCCGCCATCAACCGCCAGATCAACCTGGAGCTCTACGCCTCCTACGTTTACCTGTCCAT CCACCTCGCAGGAGCGCCAGGACTACAACCAGGACTCAGAGGCCGCCATCAACCGCCACATCAACCTGGAGCTCTGCGCCTCCTACTTTTACCTGTCCAT CACCTTCGCAGGTGCGCCAGAACTACCACCAGGATTCAGAGGCCGCCATCAACCGCCAGATCAACCCGGAGCTCTACGCCTCCTATGTTTACCTGTCCAT CCACCTCGCAGGTGCGCCAGAACTACCACCAGTACTCAGAGGCCGCATCAACCGCCAGATCAACCTGGAGCACTACGCCTCCTAAGTTTACCTGTCCCT

410

420

430

440

450

460

470

480

490

500

GTCTTACTACTTTGACCGCGATGATGTGGCTTTGAAGACTTTGCCAAATACTTTCTTCACCAATCTCATGAGGAGAGGGACATGCCGAGAACTMATG GTCTTACTACTTTGACCGCGATGATGTGGCTTTGAAGAACTTTGCCAAATACTTTCTTCACCAATCTCATGAGGAGAGGGAACATGCCGAGAAACTGATG GTCTTACTACTTTGACCGCGATGATG GGCTTTGAAGAACTTTGCCAAATACTTTCTTCACCAATCTCATGAGGAGAGGGGACATGC TGAGAAACTGATG GTCTTACTACTTTGACCGCGATGATGTGGCTTTGAAGAACTTTGCCAAATACTTTCTTCACCAATCTCATGAGGAGAGGGAACATGCTGAGAAACTGATG GTCTTACTACTTTGACCGCGATGATG TGGCTTTGAAGAACTTTGCCAAATACTTTCTTCACCAATCTCATGAGGAGAGGGAACATGCTGAGAAACTGATG GTCTTA-TACTTTGACCGCGATGATG TGCTTTGAAGAACTTTGCCAAATACTTTCTTTACCAATCTCATGAGGAGAGGGAACATGCTGAGAAACTGATG GTCTTACTACTTTGACCGTGATGACGTGGCTTTGAAGAACTTTGCCAAGTACTTTCTTCACCAATCTCATGAGGAGAGGGAACATGCCGAGAAACTGATG GTCTTACTACTTTGACCGCGATGATGTGGCTTTGAAGAACTTTGCCAAATACTTTCTTCACCAATCTCATGAGGAGAGGGACATGCTGAGAACTGATG
510
520
530
540
550
560
570
580
590

600

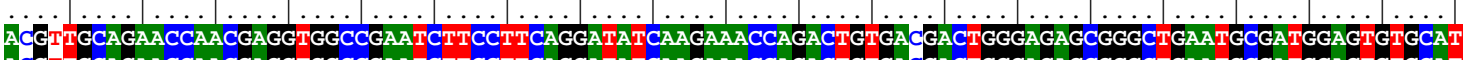
ACGTTGCAGAACCAACGAGG TGGCGAATCTTCCTTCAGGATATCAAGAAACCAGACTGT GACGACTGGGAGAGCGGGCTGAATGCGATGGAGTGTGCAT AAGCTGCAGAACCAATGAGGTGGCCGAATCTTCCTTCAGGATATCAAGAAACCAGACTGTGATGACTGGGAGAGCGGGCTGAATGCGATGGAGTGTGCAT GAGCTGCAGAACCAACTAGG TGCTTGATCTTCCTTCAGGATATCAAGAAACCAGACTGTGATGACTGGGAGAGCAGGCTGAATGCGACGGAGTGTGCAT AAGCT TCAGAACCAACGAGGTGGCCGATCTTCCTTCAGGATATCAAGAAACCAGACTGTGATGACTGGGAGAGCGGGCTGAATGCAATGGAGTGTGCAT AAGCTGCAGAACCAACGAGGTGGCCGAATCTTCCTTCAGGATATCAAGAAACCAGACTGTGATGACTGGGAGAGCGGGCTGAATGCGATGGAGTGTGCAT AAGCTGCAGAACCAACGAGGTGGCCTCATCTTCCTTCAGGATGTCAAGAAACCAGACCATGATGACTGAAAGAGCGGGCTGAATGCGATGGAGTGTGCAT AAGCT GCAGAACCAATGAGGTGGCCAATCTTCCTTCAGGATATCAAGAAACCAGACTGTGATGACTGGGAGAGCGGGTGAATGCGATGGAGTGTGCAT

$620 \quad 630$

640

650

660

670

680

690

700 $\ldots|\ldots| \ldots|\ldots| \ldots|\ldots| \ldots|\ldots| \ldots|\ldots| \ldots|\ldots| \ldots|\ldots| \ldots|\ldots| \ldots|\ldots| \ldots|\ldots| \ldots|\ldots| \ldots|\ldots|$ TACATTTGGGGAAAAAAAATATGAGTCAGTCACTACTGGAACTGCACAAACTGGCCAGTGACAAAAATGACCCCCATTTGTGTGACTTCATTGAGACAC TACATTTGG - - AAAAAAATGT GAATCAGTCACTACTGGAACTGCACAAACTGGCACTGACAAAAATGACCCCCATTTGTGTGACTT - - GAGATAC TACATTTGG - - AAAAAAATGTGAATCAGTCACTACTGGAACTGCACAAACTGGCCACTGACAAAAATGACCCCCATTTGTGTGACTTCATTGAGACAC TACATTTGG - - AAAAAAATGTGAATCAGTCACTACTGGAACTGCACAAACTGGCCACTGACAAAAATGACCCCCATTTGTGTGACTTCATTGAGACAC TATATTTGG - AAAAAAATGTGAATCAGTCACTACTGGAACTGTACAAACTGGCCACTGACAAAAATGACCCCCATTTGTGTGACTTCATTGAGACAC

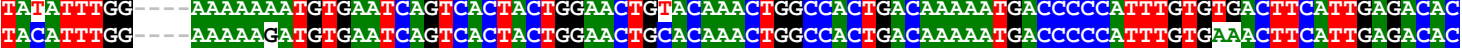
TACATTTGG

00 
Hs3

Hs3-3

Hs2

Hs13

Hs11

Hs8

TS1-psfth1

Hs2-2

Clustal Consensus

Hs3
Hs3-3
Hs2
Hs13
Hs11
Hs8
TS1-psfth1
Hs2-2
Clustal Consensus

Hs3
Hs3-3
Hs2
Hs13
Hs11
Hs8
TS1-psfth1
Hs2-2
Clustal Consensus

Hs3

Hs 3 - 3

Hs2

Hs13

Hs11

Hs8

TS1-psfth1

Hs2-2

Clustal Consensus

$\begin{array}{llllllllll}710 & 720 & 730 & 740 & 750 & 760 & 770 & 780 & 790 & 800\end{array}$ ATTACCTGAATGAGCAGGTGAAGCCATCAAAGATTGGGTGCCACGTGACCAACTTGTGCAAGATGGGAGCACCCGATCTGGCTCGGCAGAATATCT ATTACCTGAATGAGCAGGTGAAAGCCATCAAAGAATTGGGTGGCACGTGACCAACTTGTGCAAGATGGGAGCACCCGAATCTGGCTCGGCAGAATATCT ATTACCTGAATGAGCAGGTGAAAGCCATCAAAGAATTGGGTGGCCACGTGACCAACTTGTECAAGATGGGAGCACCCGAATCTGGCTCGECAGAATATC ATTACCTGAATGAGCAG ATTACCTGAATGAGCAGGTGAAAGCCATCAAAGAATTGGGTGACCACGTGACCAACTTGCGCAAGATGGGAGCGCCCGAATCTGGCTTGGCGGAATATCT ATTACCTGAAT GAGCAGGTGAAAGCCATCAAAGAATTGAGT GACCACGTGACCAACTTGCGCAAGATGGGAGCGCCCGAATCTGGCTCGGCAGAATATCT ATTACCTGAATAAGCAGGTGAAATCCATCAAAGAATTGGGTGGCCACGTGACCAACTTGCTCAAGTTGGGAGCAACTGAATCTGGCTTGGAAGAATATCT ATTACCTGAATGAGCAGGTGAAAGCCATCAAAGAATTGGT GACCACGTGACCAACTTGCGCAAGATGGGAGCGCATTACTTCCA-GAAAAGGCCTGTCA

810
$\ldots|\ldots| \ldots|\ldots| \ldots|\ldots| \ldots|\ldots| \ldots|\ldots| \ldots|\ldots| \ldots|\ldots| \ldots|\ldots| \ldots|\ldots| \ldots|\ldots| \ldots|\ldots| \ldots|\ldots| \ldots|\ldots| \ldots|\ldots| \ldots|\ldots| \ldots \mid$
CTTTGACAAGCACACGCTGGGAGACAGTGATAACGAAAGCTAAGCCTCAGGCTAATTTCCCATAGCCATGGGG GACTTCCCTGGTCACAAGGCAGT CTTTGACAAGCACACGCTGGGAGACAGTGATAACGAAAGCTAAGCCTCAGGCTAATTTCCCCATAGCCATGGGGTGACTTCCCTGGTCACCAAGGCAGT CTTTGACAAGCACACTCTGGGAGACAGTGATAATGAAAGCTAAGCCTCAGGCTAATTTCCCCATAGCCATGGGGTGACTTCCCTGGTCACCAAGGCAGT TTTTGACAAGCACATCCTGGGAGACAGTGATAATGAAAGCTAAGCCTCAGGCTAATTTCCCCATAGCCGTGGGGTGACTTCCCTGGTCACCAAGGCAGTCTTTGACAAGCACACCCTGGGAGACAGTGATAATGAAAGCTAAGCCTCGGGCTAATTTCCCCATAGCCGTGGGGTGACTTCCCTGGTCACCAAGGCAGT CTTCGACAAGCACACCCTGGGAGACAGTGATAATGAAAGCTAAGTCTCAGGCTAATTTCCCCATAGCCGTGGGGTGCCTTCCTTGGTCACCAAGGCAGT TTTGAAAGCACACCCTGGAAGACAGTGATAGCAAAAGCTAAGACTTAGGCTAATTTCCCCATAACCAAGGGGTGACTTCCCTGGTCACCAAGACAGTT ACCTGA-AAATACATGA- GCCAACCAACTATCACATTAAC-ACGATTCAG- TAG

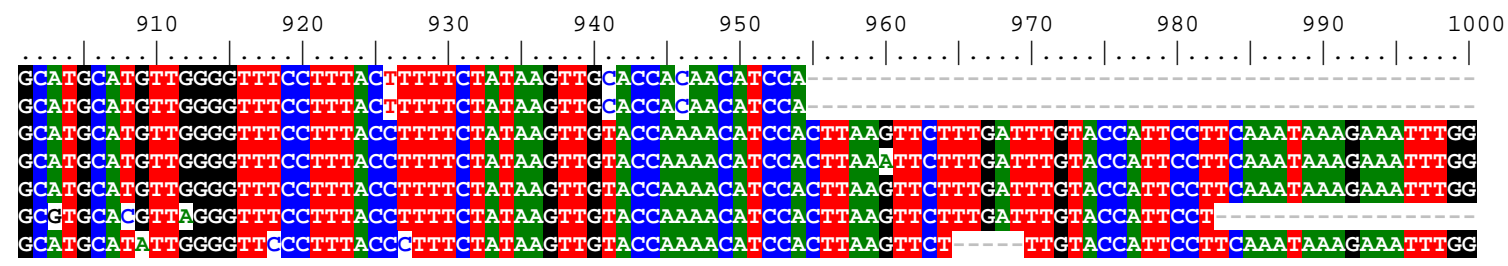
CATGCATATTGGGTTCCCTTTACCCTTTCTATAAGTTGTACCAAAACATCCACTTAAGTTCT- - TTGTACCATTCCTTCAAATAAAGAATTTGG
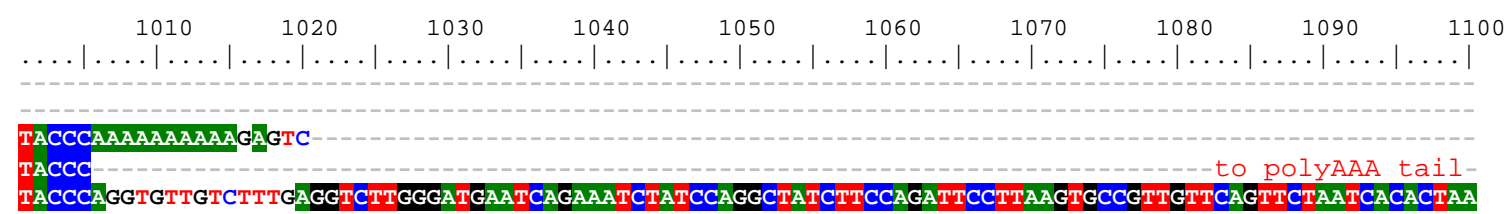

TGCCAAAAAAAAAAAAAAAAAGAAAAA

\section{Figure 5.6.3.2: Alignment of TS1-psfth1 with the expressed FTH1 called also}

\section{Hs11 of Hs, and the pseudogenes derived from it.}

There is very high identity particularly in the middle part of the molecules behind the start codon ATG, eventually coding for the $4 \alpha$-helical parts. But only $\mathrm{H} 11$, the $\mathrm{Hs}$ FTH1 has TATA-box, CAG trancription start and, like some of the pseudoferritins including Caja TS1-psfth1, the 28 nucleotide signal in the 5'FR before the translation start, ATG. Alignment has been performed between TS-psfth1, FTH1 gene, and pseudogenes Fth1 of $\mathrm{Hs}$, arabic number indicates the chromosome which the pseudogene is located (Hs11: FTH1: ID: NT033927, Hs3: ID:NT022459.1, Hs3-3: ID:NW:921651.1 , Hs2: ID:, Hs13:ID:NR002202.1, Hs-8:ID:,Hs2-2:ID:) by Clustal X. 


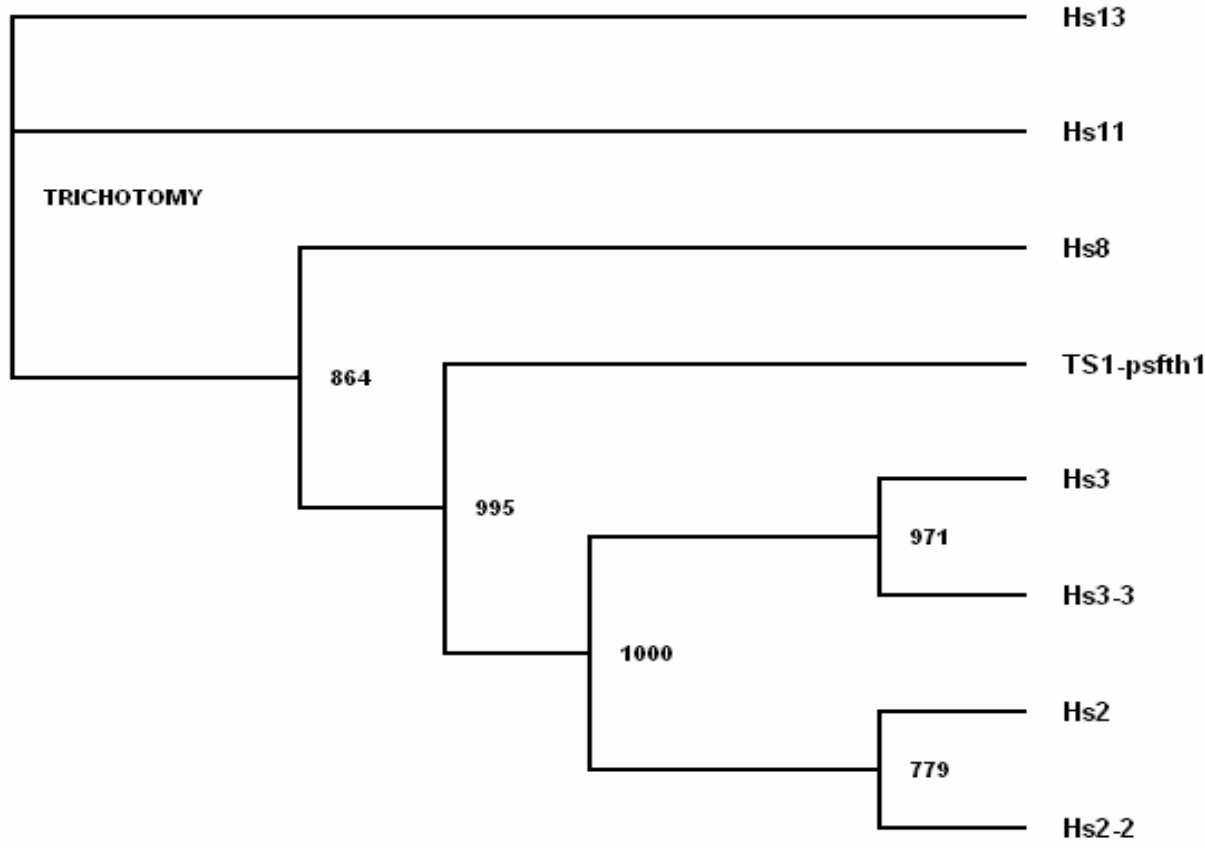

Figure 5.6.3.3: Gene tree for TS1-psfth1, the expressed FTH1 (Hs11) and the Hs pseudogenes derived from it, or a common ancestor.

(Method applied as under figure 5.5.1.1). This tree shows TS1-psfth1 closely related to Hs11, Hs13 and Hs8. Inspection of their 5'- and 3'-ends, however, shows greatest similarity between TS1-psfth1 and Hs2 (Figure 5.6.3.2). 
Coja-fth1

Gaga-fth1

Hs-Fth1

Mamu - fth1

Patr-fth1

Caja-psfth1

Rano-fth1

Mumu - fth1

Bota-fth1

Susc-fth1

Xetr-fth1

Dare-fth1

Cael-fth1

Clustal Consensus

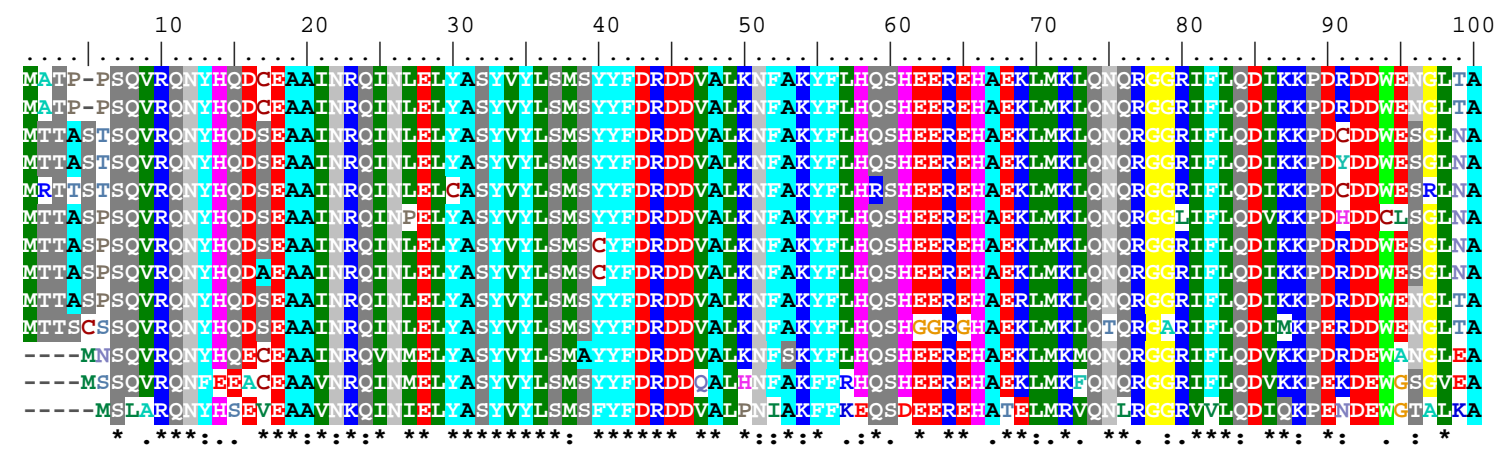

Coja-fth1

Gaga-fth1

Hs-Fth1

Mamu-fth1

Patr-fth1

Caja-psfth1

Rano-fth1

Mumu-fth1

Bota-fth1

Susc-fth1

Xetr-fth1

Dare-fth1

Cael-fth1

Clustal Consensus

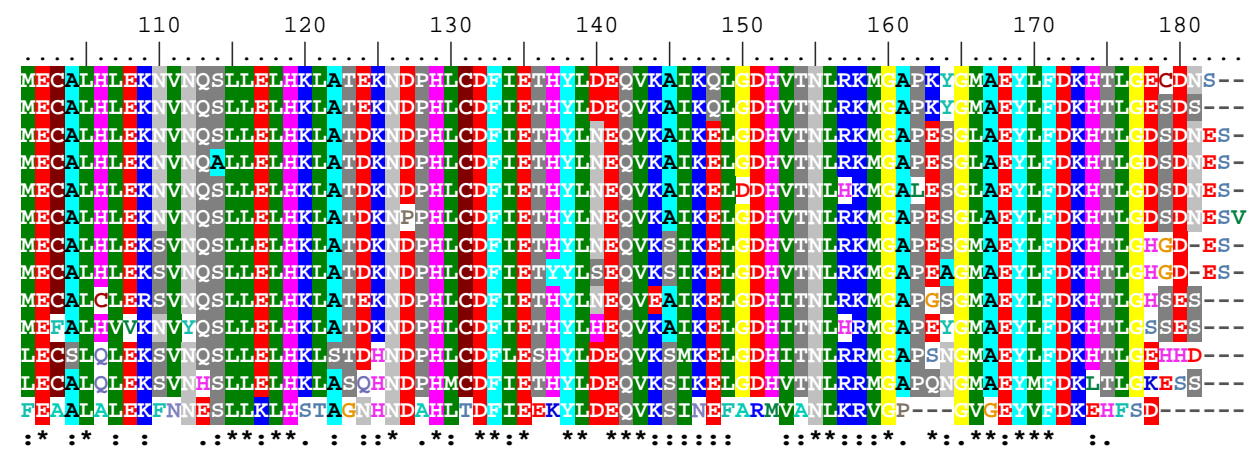

Figure 5.6.3.4: Alignment of selected apoferritin proteins, several species.

As the consensus shows (*), many apparently essential amino acids are conserved during evolution, some less when Drosophila apoferritine had been included. Aligning even apoferritins of yeast and bacterium, no amino acids in common to all are seen. Therefore, the ferritin gene of metazoa seems to have an origin different from the one of lower eukaryotes and prokaryotes; their gene product looks quite different. Note: Hs: ID: AAH70494.1, Patr: ID: XP509574.2, Mumu: ID: NP034369.1, Rano: ID: AAH78892.1, Mamu: ID: XP001104405.1, Bota. ID: NP776487.1, Susc:ID: NP999140.1, Coja: Coturnix japonica ID: AAT01287.1, Gaga: Gallus gallus ID:NP990417.1, Xetr: Xenopus tropicalis ID:NP001005135.1, Dare: Danio rerio ID:NP571660.1, Cael: Caenorhabditis elegans ID:NP491198.1. 


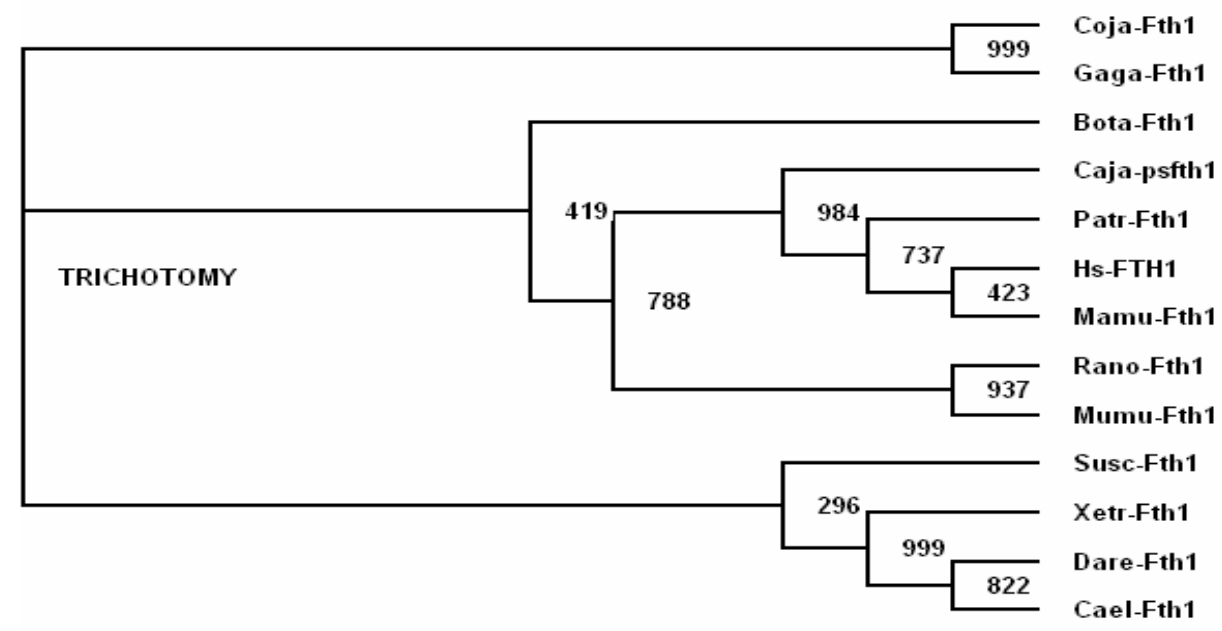

Figure 5.6.3.5: Gene tree for the evolution of animal apoferritins.

Note: the tree was constructed with amino acidic sequences as coded by expressed apoferritin genes; only a Caja pseudoferritin gene as of TS1 was compared in its formal amono acid translation produced by Swiss-prot (Method applied as under Figure 5.5.1.1).

\subsubsection{Characterization and possible interpretations of "Vorspann" $v$, the} sequence next to which a ferritin transposon has been inserted

670072-670472 585600-586000 TS1-V

Clustal Consensus

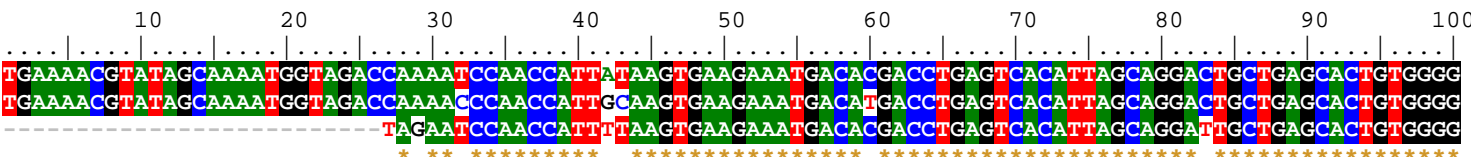

$670072-670472$ $585600-586000$ TS1-V

Clustal Consensus

$670072-670472$ 585600-586000 TS1-V

Clustal Consensus

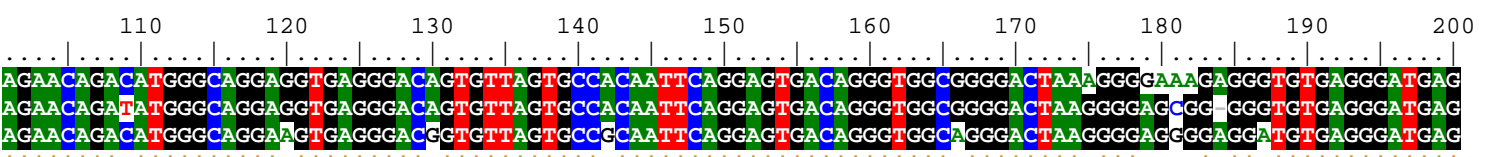

670072-670472 $585600-586000$

TS1-V AGGGGCAGAGAGAAGGGTGGAGAAGCAGGAGGTGAGGAAAGGAGCAGAGGAAAGAATTCTAAAGCAGTAGAGGAGCTGGAGGGGTTCTTTGCATT AGGGGCAGACAGAAGGGCTGGAGAGGCAGGAGG TGAGGAAAAGGAGCAGGAGAAAGAAATCTAAAGCAGTGGAGAGCCTGGCAGAGGGTTCTTTGCATT AGGGGCAAAGAGAAGGGATGGAGAAGCAGGAGGTGAGGAAAAGGGGCAGGGGAAAGAATTC

$\begin{array}{llllllllll}310 & 320 & 330 & 340 & 350 & 360 & 370 & 380 & 390 & 400\end{array}$ $\ldots \ldots|\ldots| \ldots|\ldots| \ldots|\ldots| \ldots|\ldots| \ldots|\ldots| \ldots|\ldots| \ldots|\ldots| \ldots|\ldots| \ldots|\ldots| \ldots|\ldots| \ldots|\ldots| \ldots|\ldots|$ CGGTATTTAATACATTTTGTTGGACTTCCTAAAAACTAATTGGCTCCTTATGATTAAAA - AAAAAAGAGTTACAAAAATACCAAGTGTTCAGATAAAA

Clustal Consensus

Figure 5.6.4.1: Alignment of TS1-v r-C and HCGIV-02 with the homologous Hs DNA strands of duplicons B (585 600 - 585 900) and C (670 072 - 670 432) (Hs: ID BA000025).

TS1-v r-c has less single differences with its homologue in duplicon $C$ rather than $B$ DNA. Alignment has been performed by Clustal $\mathrm{X}$. 


\section{Discussion}

The aim of this study was to describe the gene map of Callithrix jacchus' major histocompatibility complex (MHC) class I region and to elucidate its evolutionary relationship with other species. In order to establish the localization of the class I regions into the gene map BAC-Bank "CHORI-259" class I specific gene and framework probes were used to identify the clones.

The MHC class I gene complex is regarded as evolutionarily conserved because it genes have shown homology between different species: human, rat, mouse, rhesus monkey, chimpanzee and lemur (Amadou et al., 1999; The MHC sequencing consortium, 1999; Daza-Vamenta et al., 2004; Hurt et al., 2004; Anzai et al., 2003; Neff, 2005), however, Callitrhix jacchus is not known,. An improved description and understanding of the knowledge dynamics and mapping of this gene system is important for the comprehension the multiple diseases that are associated with MHC class I (Luthra-Guptasarma and Singh, 2004; Nardi et al., 2003; Feder et al., 1999; Weitkamp et al., 1994; Stein, 2001; Eslamboli, 2005)

The aim of this work was to describe a gene map of the major histocompatibility complex (MHC) class I region on Callithrix jacchus and the evolutionary relationship with other species.

In order to establish the localization of the class I regions into the gene map the BACBank "CHORI-259" was used with specific probes of class I gene and frameworks genes to identify the clones.

To elucidate the genomics structure of MHC of Callithrix jacchus is very important for the use of this animal like a model for diseases associated with the MHC (Wong et al., 2005; Pedra et al., 2005; Bach, 2005; Schulz, 2005; Lie, 2005; Reveille, 2005; Azizah et al., 2004; Shinomiya et al., 2004).

The analysis of the MHC class I in Callitrhix jacchus was constructed with three contigs represented in the BAC-clones were overlapping. The construction of the map needed the screenings of the BAC-Bank of Resources Center. The BAC-Bank contains the complete genome of Callitrhix jacchus. The genome was digested with restriction enzyme EcoRI and was cloned in the vector pTARBAC2.1. In the screening were found 256 clones class I positive of which 45 clones were selected. In them were characterized the $\mathrm{MHC}$ class I region and the framework genes that they are flanking this region, framework hypothesis (Amadou, 1999). 
153 BAC clones were found by hybridization to contain MHC class I gene(s) and/or MIC gene(s).

To determinate a genomic map for the MHC class I region of Callithrix jacchus, 42 clones were allocated to the three class I contigs.

The MHC class I gene clones were identified with a specific probes of class I and probes of framework genes. The class I probes were Caja-G04 of Callitrhix jacchus and Mamu-B of Macaca mulatta. The framework probes used came from rat and human.

The organization between class I region and framework gene showed a great similarity as has been reported in human (The MHC sequencing consortium, 1999), rat (Lambract-Washington et al., 2000; Günther and Walter, 2001), mouse (Hanson and Trowsdale, 1991), rhesus monkey (Daza-Vementa et al., 2004), chimpanzee (Kulski et al., 2005) and lemur (Neff, 2005).

The contig 1 was represented by region between framework genes BAT1 and TCF19 included class I genes. The contig 2 was not found, because there are not CAT56 positive in the hybridization analysis; however, these results are not concluding that this contig does not exist. The contig 3 was represented by region between framework genes TRIM39 and TRIM26 included class I genes. The contig 4 was represented by region between framework genes PPP1R11 and MOG.

The screening included a search for MIC genes. The major histocompatibility complex class I chain related gene A (MICA) is located near HLA-B on chromosome 6 (Bahram et al., 1994) and is expressed by keratinocytes and epithelial cells and interacts with gamma-delta T cells (Christmas et al., 1993) and is implicated in the induction of stress (Groh et al., 1996). MICA encodes molecules similar to MHC class I antigens and may share the capacity to bind peptides or other short ligands (Koldovsky et al., 1995; Bahram et al., 1994). The MICA is in the lignand of NKG2D receptor of the NK cells (Bauer et al., 1999). The MIC genes have been found in rhesus monkey (Seo et al., 1994 and Seo et al., 2001) and chimpanzee (Kulski et al., 2005).

The probes Caja-G and HLA-B have proven, quite similarly, to be very useful for a screening of DNA containing class I genes and their pseudogenes: in Callithrix jacchus, Microcebus murinus and other mammals. For this very reason, however, these probes are not very suited for mapping an order of several or even many of these similar sequences of class I genes. The major shortage is a too small number 
of discernible fragments; probably, more clones should also have been used for more steps in their lengths. So, we needed ancillary evidence for the identification of HLApositive fragments. We took fragments from other primates, Hs, where gene contents of the fragments may be concluded from knowledge of the order and bp defined locations of genes along the DNA. All fragments found could be assigned in the map, some unique lengths interpreted as ends of the inserts cleaved.

These results are in agreement with the expectation of a similar organization of the MHC class I region in Callithrix, rat and human (Hurt et al., 2004 and Shiina et al., 2003), including the rule of clusters of class I genes being placed between non-class I framework genes.

These framework genes had not been mapped before in the Common marmoset: ATP6V1g2, BAT1, TCF19, POU5F1, TRIM39, TRIM26, TRIM10, TRIM15, TCTEX5, TCTEX4 and MOG. Four contigs or intervals, respectively, showed clearly three segments containing class I genes: BAT1 - TCF19, CAT56 - TRIM39 and TCTEX4 MOG.

\subsection{Physical map of the intervals of MHC class-I}

\subsubsection{Physical map of the interval BAT1 - TCF19 (contig 1)}

Between the framework genes BAT1 and POU5F1-TCF19 has been localized contig 1 as part of the MHC class I region, in human with a $418 \mathrm{~kb}$ (The MHC consortium 1999), H2-complexes in mouse with 300 kb, as RT1-complex in rat with 450 kb (Hurt, et al., 2004) and in Patr chimpanzee (Fukami-Kobayashi, et al., 2005). These framework genes were found to be orthologous. In human, this interval contains the MICA and MICB genes (Bahram et al., 1994), HLA-B and HLA-C. The MHC class I genes expressed in this New World primate are not orthologous to any of the classical MHC class I loci of the Catarrhini $(A, B$, or $C$ loci), instead, they are most similar to the human non-classical HLA-G (Watkins, et al., 1990). This MHC class I region between BAT1 and POU5F1 has a length of approximately $350 \mathrm{~kb}$.

The contig 1 was established between BAT1 and POU5F1 by EcoRI fragments with specific hybridization of probes Caja-G, MICA, Mamu-B and respective framework genes (including ATP6Vg1 and TCF19). 


\subsubsection{Physical map of the intervals CAT56 - TRIM39 and TRIM39 - TRIM26 (contig 2 and contig 3 respectively)}

The intervals CAT56 - TRIM39 and TRIM39 - TRIM26 of the MHC class I region have polymorphic margins in human. They both belong to contig 3 in the human, where contig 2 is the longest of the four contigs, however, almost devoid of class I genes. The small numbers of clones investigated in this area did not really allow constructing any one of the contigs 2 - 4, i.e. a share of stepwise overlapping clone inserts with sufficiently many, short and well discernible fragments covering the segment between its framework borders. The intervalls expected by comparison with $\mathrm{Hs}$ and other mammals seem to be existing, however, as evidenced by positive screening of clones with the probes Caja-G, CAT56, TRIM39 and TRIM26. In this screening of the intervall CAT56 - TRIM39, 20 positive clones were found. For intervall TRIM39 TRIM26, 21 positive clones were detected.

The Interval Cat56 - TRIM39 contains class I gene(s) between the conserved framework genes CAT56 and TRIM39, with approximately of $500 \mathrm{~kb}$ in case of the RT1-complex of rat (Hurt et al., 2004).

The second intervall contains class I gene(s) between the conserved framework genes TRIM39 and TRIM26 with approximately of $200 \mathrm{~kb}$ in the RT1-complex. Near TRIM26, towards contig 4 are TRIM15 and TRIM10 (Hurt, et al., 2004). For practical reasons, the framework gene TCTEX5 located near TCTEX4 within less than $50 \mathrm{~kb}$, was included in contig 4 presumed by comparison. TCTEX 5 considered as end of a contig 3 detected clones with the framework genes TRIM 26 and TRIM39. So, there is probably no gap in Callithrix between its presumable contigs 3 and 4 (Hurt, et al., 2004; Jones, et al., 1999; Zhang, et al., 1998; Amadou, et al., 1999).

\subsubsection{Physical map of interval TCTEX4 and MOG (contig 4)}

Contig 4 contains many MHC class I genes and pseudogenes intercalated with many other genes between the conserved frameworks genes TCTEX4 and MOG. The interval has remarkably different lengths in the mammals investigated, approximately $50 \mathrm{~kb}$ in the rat RT1-complex (Hurt et al., 2004) and $400 \mathrm{~kb}$ in contig 4 of Hs. This interval is conformed by 2 clones, however, the sequence analysis support that this contig exist in Callithrix jacchus. 


\subsection{Sequence analysis of BAC clones}

Sequence analysis and hybridization DNA of BAC-clones of Callithrix jacchus have demonstrated that the organization of $\mathrm{MHC}$ class I gene and region are similar at other mammals for instance Hs, Mamu, Rano, Patr, etc. However, we have found some particular difference, like ferritine sequence, inverted sequence and pseudogene sequence.

\subsubsection{BAT1 gene in Callithrix jacchus.}

K19161C12-TS2 has shown alignment with BAT1 gene from Hs, this gene is coding to an essential splicing factor (Fleckner et al., 1997). BAT1 gene contains 10 exons (aprox $10 \mathrm{~kb}$ ) (Peelman et al., 1995), Caja sequence has shown from intron 4 until intron 6, the sequences differences between $\mathrm{Hs}$ and Caja include nucleotide substitutions and insertions/deletions; 7 insertions and 14 deletions (Figure 5.4.2). The localization of BAT1 gene is conserved in Hs and Susc (pig) (Spies, et al., 1989), K19161C12-TS2 sequence suggests is also conserved in Callithrix jacchus. Phylogenetic analysis performed between several species showed that this gene is conserved, however, the most homology was between Hs and Patr; Caja was localized nearly to Mamu and Susc (Figure 5.5.2.1).

\subsubsection{TCF19 gene in Callithrix jacchus}

The hybridization analysis was negative to the sonde of TCF19, however, the K28347D1-TCF19 sequence showed homology with human TCF19 gene into the nucleotide and amino acid sequence (Figure 5.4.4.1 and 5.4.4.2). The gene TCF19 encoded a 359 amino acid polypeptide; into K28-347D1-TCF19 amino acid sequence 72 amino acid in position 157 until 239 have shown a high homology. The transcript of this gene has seen in Mumu, Rano, Susc, Bota and Patr (Ku, et al., 1991). Phylogenetic analysis has shown that this gene is conserved between these species, however, the most homology was between Hs and Patr; Caja was localized nearly to Mamu and Susc (Figure 5.5.2.2).

\subsubsection{MOG gene in Callithrix jacchus}

The human myelin oligodendrocyte has been found in several species. The complete nucleotide sequence and structural characterization is located in chromosome 6 p22 - p21.3, with eight exons and approximately $100 \mathrm{~kb}$, telomeric to HLA-F (Amado,u et 
al., 1995; Pham-Dinh et al., 1993; Roth et al., 1995). MOG gene organization is very similar in both Hs and Mumu (Pham-Dinh et al., 1995). The MOG gene sequence of Callithrix jacchus reported here suggests a possible similarity with other species and not many evolutionary changes seem to have taken place.

\subsection{On alignment of sequences in general and particular those of Callithrix jacchus}

Most of the 55 sequences seem to be localized as listed in table 5.4.1.1. The rest although more difficult, should be solved, too. One of apparently few differences between $\mathrm{Hs}$ and Caja in class I region was the MHC ferritin pseudogene. The question of Caja with duplicon $B$ and $C$ is answered by two linear of evidences: Caja HLA-B related sequences were all found more similar with duplicon $B$ sequences in Hs; TS1 sequences, however, are generally more similar to $C$ sequences. Therefore, Caja should have both, $\mathrm{a}-\mathrm{B}$ and $\mathrm{a}-\mathrm{C}$ duplicon.

\subsection{On duplicons in the MHC class I region}

Duplicon in the MHC class I region have been studied recently by Kulsi, et al., 2004, concentrating on the $\alpha$-block (HLA section in contig 4). Although the youngest class I duplicon B and C in many Mamu instead 14 B duplicons are known (FukamiKobayashi et al., 2005). The duplicon B of Hs contains HCGIV-01 pseudogene and the duplicon C contains HCGIV-02 pseoudogene (Shiina et al., 2001). TS1 was aligned into $\mathrm{MHC}$ class I duplicon $\mathrm{B}$ and $\mathrm{C}$ with a high homology. We are assigned ps1 and ps2 like pseudogenes, because they fulfill some of the criteria proposed by Zang et al., 2004. However, it is difficult to decide whether the ps1 and ps2 specifically belong at type 1, 2 or 3, but they have the characteristics of class I pseudogenes, which are: complete genes are prevented by frameshifts, premature stop codons, fragments lacking or other defects (Hughes, et al,. 1995).

So in this area, the very young of class I duplicons, Caja contains the pseudogenes known from TS1 (ps1 and ps2) which should be useful indicators. More careful investigation must decide, if these two ancient genes might not have been originated by a duplication. 


\subsection{Transposons, particularly the pseudo ferritin found in MHC Caja transposon and its role in MHC evolution}

TS1-ps fth1 should have been transposed by an mRNA from the expressed ferritin gene from Caja, may its reincorporation into the genome reversion of the transcription process. (Perhaps its reincorporation into the genome was some sort of a reversion of a transcription process).

TS1-ps fth1 was aligned with all mRNA (gene and pseudogenes) from FTH1 of Hs. The TS1ps fth1 has the start codon and poly A end, but does not have introns, which suggests that is a processed pseudogene type 2 (Zhang et al,. 2004). Gatti et al., (1987) found pseudogenes of FTH1 in chromosome 1 - 6, 8, 9, 11, 13, 14, 17 and X. Strangely, it does contain the 28 nucleotide signal of ferritin genes but in a fragmented fashion (Murray et al., 1987). This observation requires further investigation, particularly in other locations expected to be homologous. On the other hand, there is a part of $v r$-c that does seem to be more frequently conserved, even within pseudogenes.

A simpler hypothesis that can be proposed is that the insert is placed at random. In this case, TS1-ps2 and $\mathrm{v} r-\mathrm{c}$ would be part of the whole cluster of class I pseudogenes aligning with $\vee r-c=$ HCGIV-02, suggesting the possibility that much of these is ancient 5'FR, as is actually suggested by an ATG general placed towards their 3' ends.

\subsection{On the potential value of sequence TS1 for molecular immunogenetics}

A couple of class I pseudogenes have being completely sequenced, both of which are representative examples of the many duplicons that exist in the $B / C$ section in various primates (Kulski et al., 2004). The earliest B and C duplicons contain highly identical DNA. Should the principles of duplication and mutation have been shaping the MHC from its very beginning, eventually, these probes would have to show a lot more duplicons. However, due to the degree of percentages with much less identity present in this study, this requires more sophisticated statistical evaluations beyond the scope of the present work. In any case, this underscores one of the potential uses of ps1 and ps2.

The analysis of the duplicon structure and organization suggests the presence of block duplicon model. 
Comparative analysis of MHC class I processed pseudogene sequence from new world primates: Saguinus oedipus (Saoe-PS1: ID: U52113) and Saguinus mystax (Samy-PS2 ID: U52121) (Cadavid et al., 1997) did not show identity with Callithrix jacchus ps1 and ps2.

This study presented the pioneer work about the organization of the MHC class I complex in the New World monkey Callithrix jacchus. In addition, several sequences which have never been reported before for this species have been identified. Such is the case of a ferritin pseudogene, the ps1 and ps2 pseudogenes and an inversion sequence.

Throughout the development of the present investigation, it has become apparent that Callithrix jacchus should be an excellent candidate animal model for studies of MHC complex related diseases. 


\section{Summary}

Analysis of MHC class I region in common marmoset (Callithrix jacchus) was made with 42 BAC clones. These clones containing class I region genes were identified for the purpose of both restriction mapping and PCR sequencing. Contigs 1,3 and 4 of BAC clones were obtained for genomic analysis by comparison of EcoRI fragments characterized by Southern blot fingerprint hybridization with framework and class I gene probes. A gene map for the interval BAT1 - PUO5F1 was constructed by comparison of the Caja EcoRI fragments so characterized with the computer-split $E c O R I$ fragments of the Hs class I region having known gene assignments. Ancillary evidence needed for identification of a fragment obtained from six BAC clones was obtained by analysis of sections of the Hs DNA sequence near POU5F1 for class I pseudogenes using a collection of all Hs class I pseudogene sequences for comparison of their alignment by the neighbour-joining program. Seven new pseudogenes were thereby detected within only $50 \mathrm{~kb}$ from POU5F1, each one classified by its greatest similarity with a known pseudogene. Adequately refined, this method might be useful for finding and classifying all of the many class I pseudogenes.

A genetic typification of 42 BAC clone inserts by PCR with various primers was performed. This knowledge was used for pilot sequencing in the class I region of Caja. The resulting 55 sequences were localized and potentially gene-assigned by comparative alignment, generally with DNA of $\mathrm{Hs}$, in many cases also of other primates and mammals. These sequence localizations and assignments were defined by their first and last bp numbers on the scales of Shiina and also of the Venter genome. For survey, they were both tabulated and entered into a to-bp-scale map drawn for the Hs class I region.

TS1, one of the two Caja sequences established by Prof. Takashi Shiina using a BAC clone selected by the author, was shown to contain within its 2994 bp four different pseudogenes of peculiar interest: on the right hand strand, two class I pseudogenes (ps1 and ps2); on the left hand strand, reading anti-parallel, a rather young ferritine heavy chain pseudogene (psfth1) apparently unique to Caja; and next to it exist a "Vorspann" $v$, potentially an ancient 5'FR (flanking region) which in its reverse-complementary, the right hand strand turns out to be the known pseudogene HCGIV-02, as a very highly identical, almost completely coincident sequence. The two new pseudogenes, ps1 and ps2, were recognized as being useful indicators for 
the duplicated DNA as found in the two duplicons B and C of Hs and Patr that contain the ps1, ps2 and v homologues near the classical HLA genes B and C, respectively. Alignment of ps1, ps2 and $v$ could be observed as well in all of the 14 Mamu B duplicons of the rhesus monkey Macacca mulatta. High identity alignment of ps1, ps2 and $v$ is being searched for in the vicinities of other classical HLA genes being conditions for duplicons.

Physical mapping and sequence analysis of the MHC class I region of Callithrix jacchus is to supply new genomic basic data, which further analyses of the MHC region for evolution of $\mathrm{MHC}$, disease susceptibility, immune reactivity and transplantation repulsion of the MHC to make possible. Additionally the map offers the basis for following Sequencing of the BAC clones of the individual contigs. 


\section{References}

Abi-Rached, L., Gilles, A., Shiina, T., Pontarotti, P. and Inoko, H. 2002. Evidence of en bloc duplication in vertebrate genomes. Nat. Genetic. 31, 100-105.

Adams, E.J. and Parham, P. 2001. Species-specific evolution of MHC class I genes in the higher primates. Immunol Rev. 183, 41-64.

Allcock, R.J., Price, P., Gaudieri, S., Leelayuwat, C., Witt, C.S. and Dawkins, R.L. 1999. Characterization of the human central MHC gene, BAT1: genomic structure and expression. Exp. Clin. Immunogenet. 16, 98-106.

Allcock, R.J., Williams, J.H. and Price, P. 2001. The central MHC gene, BAT1, may encode a protein that down-regulates cytokine production. Genes Cells 6, 487-494.

Alper, C.A., Awdeh, Z. and Yunis, E.J. 1992. Conserved extended MHC haplotypes. Experimental \& Clinical Immunogenetics 9, 58-71.

Alvarez, M., Martinez-Laso, J., Garcia-Torre, C., Varela, P., Perez-Blas, M., Moreno, M.A., Gomez-Casado, E., Arnaiz-Villena, A. 1998. Description of a new kind of MHC DNA sequence in Saguinus oedipus (cotton-top tamarin). Eur. J. Immunogenet. 25, 287-292.

Alvarez, M., Martinez-Laso, J., Varela, P., Diaz-Campos, N., Gomez-Casado, E., Vargas-Alarcon, G., Garcia-Torre, C. and Arnaiz-Villena, A. 1997. High polymorphism of Mhc-E locus in non-human primates: alleles with identical exon 2 and 3 are found in two different species. Tissue Antigens 49, 160.

Alvarez-Tejado, M., Martinez-Laso, J., Garcia-de-la-Torre, C., Varela, P., Recio, M.J., Allen, L., Gomez-Casado and E., Arnaiz-Villena, A. 1998. Description of two Mhc-C-related sequences in the New World monkey Saguinus oedipus. Eur. J. Immunogenet. 25, 409-417.

Amadou, C. 1999. Evolution of the Mhc class I region: the framework hypothesis. Immunogenetics; 49 4:362-367.

Amadou, C., Ribouchon, M.T., Mattei, M.G., Jenkins, N.A., Gilbert, D.J., Copeland, N.G., Avoustin, P. and Pontarotti, P. 1995. Localization of new genes and markers to the distal part of the human major histocompatibility complex $(\mathrm{MHC})$ region and comparison with the mouse: new insights into the evolution of mammalian genomes. Genomics 26, 9-20. 
Ando, A., Shigenari, A., Kulski, J.K., Renard, C., Chardon, P., Shiina, T. and Inoko, H. 2005. Genomic sequence analysis of the 238-kb swine segment with a cluster of TRIM and olfactory receptor genes located, but with no class I genes, at the distal end of the SLA class I region. Immunogenetics 57, 864873.

Anholts, J.D.H. 2002. Four new HLA alleles found, and conformation of one Class I and two Class II alleles. Thesis Department of Immunohematology and Bloodtransfusion, University Hospital of Leiden, Leiden, Netherlands.

Antunes, S.G., de Groot, N.G., Brok, H., Doxiadis, G., Menezes, A.A., Otting, N. and Bontrop, R.E. 1998. The common marmoset: a new world primate species with limited Mhc class II variability. Proc. Natl. Acad. Sci. USA 95, 11745-11750.

Anzai, T. 2003. Comparative sequencing of human and chimpanzee $\mathrm{MCH}$ class I regions unveils insetions /deletions as the major path to genomic divergence. Proc. Natl. Acad. Sci USA 100, 7708-7713.

Anzai, T., Shiina, T. and Inoko, H. 2004. Rhesus macaque class I duplicon structures, organization, and evolution within the alpha block of the major histocompatibility complex. Mol. Biol. Evol. 21, 2079-2091.

Anzai, T., Shiina, T., Kimura, N., Yanagiya, K., Kohara, S., Shigenari, A., Yamagata, T., Kulski, J.K., Naruse, T.K., Fujimori, Y., Fukuzumi, Y., Yamazaki, M., Tashiro, H., Iwamoto, C., Umehara, Y., Imanishi, T., Meyer, A., Ikeo, K., Gojobori, T., Bahram, S., Inoko, H. 2003. Comparative sequencing of human and chimpanzee MHC class I regions unveils insertions/deletions as the major path to genomic divergence. Proc. Natl. Acad. Sci. USA 100, 7708-7713.

Arnaiz-Villena, A., Martinez-Laso, J., Castro, M.J., Morales, P., Moscoso, J., Varela, P., Gomez-Casado, E. and Allende, L.M. 2001 The evolution of the MHC-G gene does not support a functional role for the complete protein. Immunol Rev. 183, 65-75.

Arnaiz-Villena, A., Morales, P., Gomez-Casado, E., Castro, M.J., Varela, P., RojoAmigo, R. and Martinez-Laso, J. 1999. Evolution of MHC-G in primates: a different kind of molecule for each group of species. J. Reprod. Immunol. 43,111-125. 
Ausubel, F.M., Brent, R., Kingston, R.E., Moore, D.D., Seidman J.G., Smith J.A. and Struhl, K. 1994-2005. Current protocols in molecular biology. 1 - 5 vols. Different Chapter.

Azizah, M.R., Kuak, S.H., Ainol, S.S., Rahim, M.N., Normaznah, Y. and Norella, K. 2004. Association of the tumor necrosis factor alpha gene polymorphism with susceptibility and clinical-immunological findings of systemic lupus erythematosus. Asian Pac. J. Allergy Immunol. 22, 159-163.

Bach, J.F. 2005. [Infections and autoimmunity]. Rev. Med. Interne. 1, 32-34.

Beck, S. and Trowsdale, J. 2000. The human major histocompatability complex: lessons from the DNA sequence. Annu. Rev. Genomics Hum. Genet. 1, 117137.

Beck, T. 2005. The feline major histocompatibility complex is rearrenged by an inversion with a breakpoint in the distal class I region. Immunogenetics 56, 702-709.

Begovich, A.B., McClure, G.R. and Suraj, V.C. 1992. Polymorphism, recombination, and linkage disequilibrium within the HLA class II region. J. Immunology 148, 249-258.

Bergstrom, T.F., Erlandsson, R., Engkvist, H., Josefsson, A., Erlich, H.A. and Gyllensten, U. 1999. Phylogenetic history of hominoid DRB loci and alleles inferred from intron sequences. Immunol. Rev. 167, 351-365.

Bingulac-Popovic, J., Figueroa, F., Sato, A., Talbot, W.S., Johnson, S.L., Gates, M., Postlethwait, J.H. and Klein, J. 1997. Mapping of mhc class I and class II regions to different linkage groups in the zebrafish, Danio rerio. Immunogenetics 46, 129-134.

Bowen, M.A., Bajorath, J., D'Egidio, M., Whitney, G.S., Palmer, D., Kobarg,J., Starling,G.C., Siadak, A.W. and Aruffo, A. 1997. Characterization of mouse ALCAM (CD166): the CD6-binding domain is conserved in different homologs and mediates cross-species binding. Eur. J. Immunol. 27, 1469-1478.

Boyson, J.E., Iwanaga, K.K., Urvater, J.A., Hughes, A.L., Golos, T.G. and Watkins, D.I. 1999. Evolution of a nonclassical MHC class I locus in two Old World primate species. Immunogenetics 49, 86-98. 
Boyson, J.E., Shufflebotham, C., Cadavid, L.F., Urvater, J.A., Knapp, L.A., Hughes, A.L. and Watkins, D.I. 1996. The MHC class I genes of the rhesus monkey. Different evolutionary histories of MHC class I and II genes in primates. J. Immunol. 156, 4656-4665.

Brok, H.P., Uccelli, A., Kerlero De Rosbo, N., Bontrop, R.E., Roccatagliata, L., de Groot, N.G., Capello, E., Laman, J.D., Nicolay, K., Mancardi, G.L., Ben-Nun, A., Hart, B.A. 2000. Myelin/oligodendrocyte glycoprotein-induced autoimmune encephalomyelitis in common marmosets: the encephalitogenic T cell epitope pMOG24-36 is presented by a monomorphic MHC class II molecule. J. Immunol. 165, 1093-1101.

Bronson, S.K., Pei, J., Taillon-Miller, P., Chorney, M.J., Geraghty, D.E. and Chaplin, D.D. 1991. Isolation and characterization of yeast artificial chromosome clones linking the HLA-B and HLA-C loci. Proc. Natl. Acad. Sci. USA 88, 1676-1680.

Cadavid, L.F., Hughes, A.L., Watkins, D.I. 1996. MHC class I-processed pseudogenes in New World primates provide evidence for rapid turnover of MHC class I genes. J. Immunol. 157, 2403-2309.

Cadavid, L.F., Mejia, BE, Watkins DI. 1999. MHC class I genes in a New World primate, the cotton-top tamarin (Saguinus oedipus), have evolved by an active process of loci turnover. Immunogenetics 49, 196-205.

Cadavid, L.F., Shufflebotham, C., Ruiz, F.J., Yeager, M., Hughes, A.L. and Watkins, D.I. 1997. Evolutionary instability of the major histocompatibility complex class I loci in New World primates. Proc. Natl. Acad. Sci. USA 94, 14536-14541.

Caggiari, L., De Re, V. and Rehermann, B. 2006. Identification of novel chimpanzee MHC I and II alleles using an improved sequence-based typing strategy. Hum. Immunol. 67, 63-72.

Canavez, F.C., Moreira, M.A., Ladasky, J.J., Pissinatti, A., Parham, P. and Seuanez H.N. 1999. Molecular phylogeny of new world primates (Platyrrhini) based on beta2-microglobulin DNA sequences. Mol. Phylogenet. Evol. 12, 74-82. 
Cardenas, P.P., Suarez, C.F., Martinez, P., Patarroyo, M.E. and Patarroyo, M.A. 2005. MHC class I genes in the owl monkey: mosaic organization, convergence and loci diversity. Immunogenetics 56, 818-832.

Castellino, F. 1997. Antigen presentation by MHC class II molecules: invariant chain function, protein trafficking, and the molecular basis of diverse determinant capture. Hum. Immunol. 54, 159-169.

Chardon, P., Renard C., Gaillard, C.R. and Vaiman, M. 2000. The porcine major histocompatibility complex and related paralogous region. Genet. Sol. Evol. $32,109-128$.

Christmas, S.E., Brew, R., Deniz, J. and Taylor, J.J. 1993. T cell receptor heterogeneity of gamma delta $\mathrm{T}$ cell clones from human female reproductive tissues. Immunology 78, 436-442.

Chu, T.W., Capossela, A., Coleman, R., Goei, V.L., Nallur, G. and Gruen, J.R. 1995. Cloning of a new 'finger' protein gene (ZNF173) within the class I region of the human MHC. Genomics 29, 229-239.

Coimbra-Filho, A.F. 1984. Situacao Atul Dos Calitriquideos que Ocorrem no Brasil (Callitrichidae-Primates). in A Primatologia no Brasil. ed. M.T. de Mello. Sociedade Brasileira de Primatologia: Brasilia.

Costanzo, F., Colombo, M., Staempfli, S., Santoro, C., Marone, M., Frank, R., Delius, H. and Cortese, R. 1986 Structure of gene and pseudogenes of human apoferritin H. Nucleic Acids Res. 14, 721-736.

Dagenais, S.L. and Nakamura, I. 1997. A physical map of the mouse H2 Bat5/Db interval. Mamm. Genome 8, 39-41.

Daza-Vamenta, R., Glusman, G., Rowen, L., Guthrie, B. and Geraghty, D.E. 2004. Genetic divergence of the rhesus macaque major histocompatibility complex. Genome Res. 14, 1501-1515.

Degli-Esposti, M.A., Leaver, A.L., Christiansen, F.T., Witt, C.S., Abraham, L.J. and Dawkins, R.L. 1992. Ancestral haplotypes: conserved population MHC haplotypes. Human Immunology 34, 242-252.

Diaz, D., Naegeli, M., Rodriguez, R., Nino-Vasquez, J.J., Moreno, A., Patarroyo, M.E., Pluschke, G. and Daubenberger, C.A. 2000. Sequence and diversity of 
MHC DQA and DQB genes of the owl monkey Aotus nancymaae. Immunogenetics 51, 528-537.

Digby, L. 1995. Infant Care, Infanticide, and Female Reproductive Strategies in Polygynous Groups of Common Marmosets (Callithrix jacchus). Behav. Ecol. Sociobol. 37, 51-61.

Doxiadis, G.G., van der Wiel, M.K., Brok, H.P., de Groot, N.G., Otting, N., 't Hart, B.A., van Rood, J.J. and Bontrop, R.E.. 2006. Reactivation by exon shuffling of a conserved HLA-DR3-like pseudogene segment in a New World primate species. Proc. Natl. Acad. Sci. USA 103, 5864-5868.

Dunham, I., Sargent, C.A., Dawkins, R.L., Campbell, R.D. 1989. An analysis of variation in the long-range genomic organization of the human major histocompatibility complex class II region by pulsed-field gel electrophoresis. Genomics 5, 787-796.

Epple, G. 1970. Maintenance, breeding, and development of marmoset monkeys (callitrichidae) in captivity. Folia Primat. 12, 56-76.

Epple, G., Belcher, A.M., Kuderling, I., Zeller, U., Scolnick, L., Greenfield, K.L., Smith III, A.B. 1993. Making sense out of scents: species differences in scent glands, scent-marking behaviour, and scent-mark composition in the callitrichdae. In marmosets and tamarins: systematics, behaviour, and ecology. Ed. Anthony B. Rylands, Oxford University Press.

Epple, G.1968. Comparative Vocalization in Marmoset Monkeys. Folia Primat. 8 , $1-40$.

Eslamboli A. 2005. Assessment of GDNF in primate models of Parkinson's disease: comparison with human studies. Rev Neurosci; 16, 303-310.

Evans, D.T., Knapp, L.A., Jing, P., Piekarczyk, M.S., Hinshaw, V.S. and Watkins, D.I. 1999. Three different MHC class I molecules bind the same CTL epitope of the influenza virus in a primate species with limited MHC class I diversity. J. Immunol. 162, 3970-3977.

Evans, D.T., Piekarczyk, M.S., Allen, T.M., Boyson, J.E., Yeager, M., Hughes, A.L., Gotch, F.M., Hinshaw, V.S. and Watkins, D.I. 1997. Immunodominance of a single CTL epitope in a primate species with limited MHC class I polymorphism. J. Immunol. 159, 1374-1382. 
Evans, D.T., Piekarczyk, M.S., Cadavid, L., Hinshaw, V.S. and Watkins, D.I. 1998. Two different primate species express an identical functional MHC class I allele. Immunogenetics 47, 206-211.

Evans, S. 1983. The Pair-bond of the Common Marmoset, Callithrix jacchus jacchus: An Experimental Investigation. Animal Behavior 31, 651-658.

Evans, S. and Poole, T.B. 1984. Long-term Changes and Maintenance of the Pair-bond in Common Marmosets Callithrix jacchus jacchus. Folia Primat. 42, 33-41.

Feder, J.N. 1999. The hereditary hemochromatosis gene (HFE): a MHC class Ilike gene that functions in the regulation of iron homeostasis. Immunol. Res. 20, 175-185.

Flajnik, M.F., Canel, C., Kramer, J. and Kasahara, M. 1991. Which came first, MHC class I or class II? Immunogenetics 33, 295.

Fleckner, J.; Zhang, M.; Valcarcel, J.; Green, M. R. 1997. U2AF(65) recruits a novel human DEAD box protein required for the U2 snRNP-branchpoint interaction. Genes Dev. 11, 1864-1872.

Flügge, P., Zimmermann, E., Hughes, A.L., Günther, E. and Walter, L. 2002. Characterization and phylogenetic relationship of prosimian $\mathrm{MHC}$ class I genes. J. Mol. Evol. 55, 768-775.

Fukami-Kobayashi, K., Shiina, T., Anzai, T., Sano, K., Yamazaki, M., Inoko, H. and Tateno, Y. 2005. Genomic evolution of MHC class I region in primates. Proc. Natl. Acad. Sci. USA 102, 9230-9234.

Gaudieri. S., Leelayuwat, C., Tay GK, Townend DC, Dawkins RL. 1997. The major histocompatability complex (MHC) contains conserved polymorphic genomic sequences that are shuffled by recombination to form ethnic-specific haplotypes. J. Mol. Evolution 45, 17-23.

Germain RN. 1995. The biochemistry and cell biology of antigen presentation by MHC class I and class II molecules. Implications for development of combination vaccines. Ann. NY Acad. Sci. 754, 114-125.

Giffon, T., Lepourcelet, M., Pichon, L., Jezequel, P., Bouric, P., Carn, G., Pontarotti, P., Gall, J.Y. and David, V. 1996. Cloning of a human homologue 
of the mouse Tctex-5 gene within the MHC class I region. Immunogenetics 44, 331-339.

Go, Y., Satta, Y., Kawamoto, Y., Rakotoarisoa, G., Randrianjafy, A., Koyama, N. and Hirai, H. 2003. Frequent segmental sequence exchanges and rapid gene duplication characterize the MHC class I genes in lemurs. Immunogenetics $55,450-461$.

Goei, V.L., Parimoo, S., Capossela, A., Chu, T.W. and Gruen, J.R. 1994. Isolation of novel non-HLA gene fragments from the hemochromatosis region (6p21.3) by cDNA hybridization selection. Am. J. Hum. Genet. 54, 244-251.

Gonzalez, P., Pinto, F., Nogales, M., Jimenez-Asensio, J., Hernandez, M. and Cabrera, V.M. 1996. Phylogenetic relationships of the Canary Islands endemic lizard genus Gallotia (Sauria: Lacertidae), inferred from mitochondrial DNA sequences. Mol. Phylogenet. Evol. 6, 63-71.

Goujet-Zalc, C., Faille, A., Teillaud, J.L., Turmel, P., Mahouy, G., Charron, D.J. 1984. Molecular characterization of two la-like antigens in marmoset. Immunogenetics 19, 155-161.

Graser, R., Vincek, V., Takami, K. and Klein, J. 1998. Analysis of zebrafish Mhc using BAC clones. Immunogenetics 47, 318-325.

Groh, V., Bahram, S., Bauer, S., Herman, A., Beauchamp, M. and Spies, T. 1996. Cell stress-regulated human major histocompatibility complex class I gene expressed in gastrointestinal epithelium. Proc. Natl. Acad. Sci. USA 93, 12445-12450.

Groves C. 2001. Primate taxonomy. Washington DC: Smithsonian Inst Pr. 350 p

Groves, C.P. 2001. Primate Taxonomy. Smithsonian Institution Press. Washington y Londres. 350 pages.

Gruen, J.R., Nalabolu, S.R., Chu, T.W., Bowlus, C., Fan, W.F., Goei, V.L., Wei, H., Sivakamasundari, R., Liu, Y., Xu, H.X., Parimoo, S., Nallur, G., Ajioka, R., Shukla, H., Bray-Ward, P., Pan, J. and Weissman, S.M. 1996. A transcription map of the major histocompatibility complex $(\mathrm{MHC})$ class I region. Genomics 36, 70-85. 
Grundschober, C., Labonne, M.P., Javaux, F., Steiner, Q.G., Gebuhrer, L.and Tiercy, J.M. 1998. Sequence of four new HLA-Cw alleles: a possible role of interallelic recombination. Tissue Antigens. 51, 72-79.

Ha, H., Howard, C.A., Yeom, Y.I., Abe, K., Uehara, H., Artzt, K. and Bennett, D. 1991. Several testis-expressed genes in the mouse t-complex have expression differences between wild-type and t-mutant mice. Dev. Genet. 12, 318-332.

Hall, T.A. 1999. BioEdit: a user-friendly biological sequence alignment editor and analysis program for Windows 95/98/NT. Nucl. Acids. Symp. Ser. 41, 95-98.

Handel-Fernandez, M.E., Kurimoto, I., Streilein, J.W. and Vincek, V. 1999. Genetic mapping and physical cloning of UVB susceptibility region in mice. J. Invest. Dermatol. 113, 224-229.

Hansen, A. 2004. Bioinformatik ein Leitfaden für Naturwissenschaftler 2. überarbeitete und erweiterte Auflage. Birkhäuser. Germany.

Harada, H., Harada, Y., O'Brien, D.P., Rice, D.S., Naeve, C.W. and Downing, J.R. 1999. HERF1, a novel hematopoiesis-specific RING finger protein, is required for terminal differentiation of erythroid cells. Mol. Cell. Biol. 19, 3808-3815.

Hartl, D.L. 2000. A primer of population genetics. 3rd ed. Sinauer Associates, USA.

Hauptmann, G. and Bahram, S. 2004. Genetics of the central MHC. Curr Opin Immunol. 16, 668-672.

Heeney, J.L., van Els, C., de Vries, P., ten Haaft, P., Otting, N., Koornstra, W., Boes, J., Dubbes, R., Niphuis, H., Dings, M., Cranage, M., Norley, S., Jonker, M., Bontrop, R.E. and Osterhaus, A. 1994. Major histocompatibility complex class I-associated vaccine protection from simian immunodeficiency virusinfected peripheral blood cells. J. Exp. Med. 180, 769-774.

Helou, K., Yan, Q., Yuan, X.J., Kunz, H.W., Levan, G. and Gill, T.J. III. 1999. Cytogenetic localization of the growth and reproduction complex (Grc) in the rat and in the mouse and its position in relation to RT1.EC and other loci in the rat MHC. Hereditas 130, 105-109.

Henry, J., Ribouchon, M., Depetris, D., Mattei, M., Offer, C., Tazi-Ahnini, R. and Pontarotti, P. 1997. Cloning, structural analysis, and mapping of the B30 and 
B7 multigenic families to the major histocompatibility complex (MHC) and other chromosomal regions. Immunogenetics 46, 383-395.

Herberg JA, Sgouros J, Jones T, Copeman J, Humphray SJ, Sheer D, Cresswell P, Beck S and Trowsdale J. 1998. Genomic analysis of the Tapasin gene, located close to the TAP loci in the MHC. Eur. J. Immunol. 28, 459-467.

Hickson, R.E. and Cann, R.L. 1997. Mhc allelic diversity and modern human origins. J. Mol. Evol. 45, 589-598.

http://www.bacpac.chori.org

http://www.damisela.com/zoo/mam/primates/callitrichidae/jacchus/taxa.html

http://www.ebi.ac.uk/emboss/transeq/

http://www.ensembl.org/index.html

http://www.expasy.org/

http://www.genome.wi.mi.edu/cgi-bin/primer/primer3.cgi.

http://www.infobiogen.fr/doc/ClustalW/clustalw.html

http://www.itis.usda.gov/servlet/SingleRpt/SingleRpt?search topic=TSN\&search value $=572915$

http://www.ncbi.nlm.nih.gov/BLAST/

http://www.ncbi.nlm.nih.gov/entrez/query.fcgi?CMD=search\&DB=pubmed

http://www.ncbi.nlm.nih.gov/entrez/query.fcgi?db=gene

http://pin.primate.wisc.edu/factsheets/entry/common marmoset/taxon

http://www.repeatmasker.org/cgi-bin/WEBRepeatMasker

http://www.saudeanimal.com.br/sagui.html

http://www.zoo.ru/Moscow/monk e8.html

Hughes, A.L. and Nei, M. 1993. Evolutionary relationships of the classes of major histocompatibility complex genes. Immunogenetics 37, 337-346.

Hughes, A.L. and Nei, M. 1990. Evolutionary relationships of class II majorhistocompatibility- complex genes in mammals. Molecular Biology \& Evolution 7, 491-514.

Hughes, A.L. and Yeager, M. 1998. Natural selection at major histocompatibility complex loci of vertebrates. Annu. Rev. Genet. 32, 415-435. 
Hurt, P., Walter, L., Sudbrak, R., Klages, S., Mueller, I., Shiina, T., Inoko,H., Lehrach, H., Guenther, E., Reinhardt, R. and Himmelbauer, H. 2004. The genomic sequence and comparative analysis of the rat major histocompatibility complex. Genome Res. 14, 631-639.

Ioannidu, S., Walter, L., Dressel, R. and Gunther, E. 2001. Physical map and expression profile of genes of the telomeric class I gene region of the rat MHC. J. Immunol. 166, 3957-3965.

Iris, F., Bougueleret, L., Prieur, S., Caterina, D., Primas, G., Perrot, V., Jurka, J., Rodriguez-Tome, P., Claverie, J., Cohen, D. and Dausset, J. 1993. Dense Alu clustering and a potential new member of the NF kappa B family within a 90 kilobase HLA class III segment. Nat. Genet. 3, 137-145.

Janeway, C.A., Travers, P., Walport, M. and Capra, J.D. 1999. Immunobiology. 4th ed. Elservier Science London.

Jones, E.P., Kumanovics, A., Yoshino, M. and Fischer Lindahl, K. 1999. Mhc class I and non-class I gene organization in the proximal $\mathrm{H} 2-\mathrm{M}$ region of the mouse. Immunogenetics 49, 183-195.

Jones, E.Y., Fugger, L., Strominger, J.L., Siebold, C. 2006. MHC class II proteins and disease: a structural perspective. Nat. Rev. Immunol. 6, 271-282.

Jones, J.S. and Partridge, L. 1983. Tissue rejection: the price for sexual acceptance. Nature 304, 484-485.

Jones, R.A., Scott, C.S. and Child, J.A. 1988. Expression of MHC class I and class I-like gene products on the cell membrane of mature and immature $T$ cells. Leuk. Res. 12, 799-804.

Kahloun, A., Chauvel, B., Mauvieux, V., Dorval, I., Jouanolle, A.M., Gicquel, I., Le Gall, J.Y. and David, V. 1993. Localization of seven new genes around the HLA-A locus. Hum. Mol. Genet. 2, 55-60.

Kanki, J.P., Chang, S. and Kuwada, J.Y. 1994. The molecular cloning and characterization of potential chick DM-GRASP homologs in zebrafish and mouse. J. Neurobiol. 25, 831-845.

Kaufman, J., Skjoedt, K. and Salomonsen, J. 1990. The MHC molecules of nonmammalian vertebrates. Immunological Reviews 113, 83-117. 
Klein, J. and Sato, A. 1998. Birth of the major histocompatibility complex. Scan. J. Immunology 47, 199.

Klein, J. 2001. George Snell's first foray into the unexplored territory of the major histocompatibility complex. Genteric 159, 435-439.

Klein, J. 1976. Evolution and function of the major histocompatibility complex: facts and speculations. In: Gotze D, ed. The Major Histocompatibility System in Man and Animals, New York: Springer-Verlag, 339-378.

Knapp, L.A., Cadavid, L.F. and Watkins, D.I. 1998. The MHC-E locus is the most well conserved of all known primate class I histocompatibility genes. J. Immunol. 160, 189-196.

Koch, J. and Tampe, R. 2006. The macromolecular peptide-loading complex in MHC class I-dependent antigen presentation. Cell. Mol. Life Sci. 63, 653-662.

Koldovsky, P., Haas, I., Beir, H. and Ganzer, U. 1995. Immunohistochemica examination of 11 cell lines derivaded from human head and nech saqumous cell carcinomas, their recurrence or metastases. Europ. Arch. Oto-RhinoLaryngo 252, 359-365.

Krebs, K.C., Jin, Z.Y., Rudersdorf, R., Hughes, A.L. and O'connor, D.H. 2005. Unusually high frequency MHC class I alleles in mauritian origin cynomolgus macaques. J. Immunol. 175, 5230-5239.

Kriener, K., O'hUigin, C, and Klein, J. 2001. Independent origin of functional MHC class II genes in humans and New World monkeys. Hum. Immunol. 62, 1-14.

Krishnan, B.R., Jamry, I. and Chaplin, D.D. 1995. Feature mapping of the HLA class I region: localization of the POU5F1 and TCF19 genes. Genomics 30, 53-58.

Ku, D.H., Chang, C.D., Koniecki, J., Cannizzaro, L.A., Boghosian-Sell, L., Alder, H. and Baserga, R. 1991. A new growth-regulated complementary DNA with the sequence of a putative trans-activating factor. Cell Growth Differ. 2, 179186.

Kulski, J.K., Anzai, T. and Inoko, H. 2005. ERVK9, transposons and the evolution of MHC class I duplicons within the alpha-block of the human and chimpanzee. Cytogenet. Genome Res. 110, 181-192. 
Kulski, J.K., Anzai, T., Shiina, T. and Inoko, H. 2004. Rhesus macaque class I duplicon structures, organization, and evolution within the alpha block of the major histocompatibility complex. Mol. Biol. Evol. 21, 2079-2091.

Kumar, S., Tamura, K., Jakobsen, I.B. and Nei, M. 2001. MEGA2: molecular evolutionary genetics analysis software. Bioinformatics 17, 1244-1245.

Lafont, B.A.P., Buckler-White, A., Plishka, R., Buckler, C. and Martin, M.A. 2003. Characterization of pig-tailed macaque classical MHC class I genes: implications for $\mathrm{MHC}$ evolution and antigen presentation in macaques. $\mathrm{J}$. Immunol. 171, 875-885.

Lambracht-Washington, D. and Fischer Lindahl K. 2002. Does the rat have an H2-D orthologue next to Bat1? Immunogenetics 53, 1039-1046.

Lawlor, D.A, E., Taylor, P., and Parham, P. 1991. Gorilla class I major histocompatibility complex alleles: comparison to human and chimpanzee class I. J. Exp. Med. 174, 1491-1509.

Lazaro AM, Moraes ME, Marcos CY, Moraes JR, Fernandez-Vina MA, Stastny P. 1999. Evolution of HLA-class I compared to HLA-class II polymorphism in Terena, a South-American Indian tribe. Hum Immunol. 60, 1138-1149.

Lehner, B., Semple, J.I., Brown, S.E., Counsell, D., Campbell, R.D. and Sanderson, C.M. 2004. Analysis of a high-throughput yeast two-hybrid system and its use to predict the function of intracellular proteins encoded within the human MHC class III region. Genomics 83, 153-167.

Leonhardt, R.M., Keusekotten, K., Bekpen, C. and Knittler, M.R. 2005. Critical role for the tapasin-docking site of TAP2 in the functional integrity of the MHC class I-peptide-loading complex. J. Immunol. 175, 5104-5114.

Lepourcelet, M., Andrieux, N., Giffon, T., Pichon, L., Hampe, A., Galibert, F. and Mosser, J. 1996. Systematic sequencing of the human HLA-A/HLA-F region: establishment of a cosmid contig and identification of a new gene cluster within $37 \mathrm{~kb}$ of sequence. Genomics 37, 316-326.

Lie, J.T., Kobaysshi, S., Tokano,Y. and Hashimoto, H. 1995. Systemic and cerebral vasculitis coexisting with disseminated coagulopathy in systemic lupus erythematosus associated with antiphospholipid syndrome. $\mathrm{J}$. Rheumatol. 22, 2173-2176. 
Liu W, Zhao Y, Liu Z, Zhang Y, Lian Z, Li N. 2006. Construction of a 7-fold BAC library and cytogenetic mapping of 10 genes in the giant panda (Ailuropoda melanoleuca).BMC Genomics. 17, 1-8.

Lundberg AS, 1992. Evolution of major histocompatibility complex class II allelic diversity: direct descent in mice and humans. Proc. Natl. Acad. Sci. USA 89, 6545-6549.

Luo, M.-J.; Zhou, Z., Magni, K., Christoforides, C., Rappsilber, J., Mann, M. and Reed, R. 2001. Pre-mRNA splicing and mRNA export linked by direct interactions between UAP56 and Aly. Nature 413, 644-647.

Luthra-Guptasarma, B. and Singh, B. 2004. HLA-B27 lacking associated beta2microglobulin rearranges to auto-display or cross-display residues 169-181: a novel molecular mechanism for spondyloarthropathies. FEBS Lett. 575, 1-8.

Martin, R.D. 1990. Primate Origins and Evolution: A Phylogenetic Reconstruction. Princeton University Press: Princeton, N.J.

Matsunaga, T. and Rahman, A. 1998. What brought the adaptive immune system to vertebrates? Immunological Reviews 166, 177-186.

Mattiuz, P.L., Inde, D., Piazza, A., Ceppelini, R., Bodmer, W.F. 1970. New approaches to the population genetic and segregation analysis of the HLA system. In: Terasaki P, ed. Histocompatibility Testing, Copenhagen: Munksgaard, 1971, 193-205.

Miller, M.M., Goto, R., Bernot, A., Zoorob, R., Auffray, C., Bumstead, N. and Briles, W.E. 1994. Two Mhc class I and two Mhc class II genes map to the chicken Rfp-Y system outside the B complex. Proc. Natl. Acad. Sci. USA 91, 4397-4401.

Milner, C.M. and Campbell, R.D. 2001. Genetic organization of the human MHC class III region. Front. Biosci. 6, D914-926.

Mizuki, N., Ando, H., Kimura, M., Ohno, S., Miyata, S., Yamazaki, M., Tashiro, H., Watanabe, K., Ono, A., Taguchi, S., Sugawara, C., Fukuzumi, Y., Okumura, K., Goto, K., Ishihara, M., Nakamura, S., Yonemoto, J., Kikuti, Y.Y., Shiina, T., Chen, L., Ando, A., Ikemura, T. and Inoko, H. 1997. Nucleotide sequence analysis of the HLA class I region spanning the 237-kb segment around the HLA-B and -C genes. Genomics 42, 55-66. 
Nagamachi, C.Y., Pieczarka, J.C., Schwarz, M., Barros, R.M., Mattevi, M.S..1997. Chromosomal similarities and differences between tamarins, Leontopithecus and Saguinus (Platyrrhini, Primates). Am. J. Primatol. 43, 265-276.

Nair, S., Dey, R., Sanford, J.P. and Doyle, D. 1992. Molecular cloning and analysis of an elF-4A-related rat liver nuclear protein. J. Biol. Chem. 267, 12928-12935.

Nardi, G., Cadiz, C., Lachman, J. and Cornelio, C. 2003. Advances in hereditary hemochromatosis. Acta Gastroenterol. Latinoam. 33, 103-107.

Neff, J. 2005. Genomische und evolutionäre Analyse von MHC-Klasse-I-Genen bei einem Halbaffen (Microcebus murinus). Tiermedizin Hochschule Hannover. Dissertation.

Nei, M., and Gojobori, T. 1986. Simple methods for estimating the numbers of synonymous and nonsynonymous nucleotide substitutions. Mol. Biol. Evol. 3, 418-442.

Neville, M. J. and Campbell, R. D. 1999. A new member of the Ig superfamily and a V-ATPase $\mathrm{G}$ subunit are among the predicted products of novel genes close to the TNF locus in the human MHC. J. Immun. 162, 4745-4754.

Norcross, J.L. and Newman, J.D. 1993. Context and Gender-specific Differences in the Acoustic Structure of Common Marmoset (Callithrix jacchus) Phee Calls. Am. J. Primatology 30, 37-54.

Ohta, T. 1991. Role of diversifying selection and gene conversion in evolution of Major Histocompatibility Complex loci. Proc. Natl. Acad. Sci. USA 15, 67166720.

Okamoto, K., Okazawa, H., Okuda, A., Sakai, M., Muramatsu, M. and Hamada, H. 1990. A novel octamer binding transcription factor is differentially expressed in mouse embryonic cells. Cell 60, 461-472.

Orimo, A., Yamagishi, T., Tominaga, N., Yamauchi, Y., Hishinuma, T., Okada, K., Suzuki, M., Sato, M., Nogi, Y., Suzuki, H., Inoue, S., Yoshimura, K., Shimizu, Y. and Muramatsu, M. 2000. Molecular cloning of testis-abundant finger Protein/Ring finger protein 23 (RNF23), a novel RING-B box-coiled coil-B30.2 protein on the class I region of the human MHC. Biochem. Biophys. Res. Commun. 276, 45-51. 
Osoegawa, K., Vessere, G.M., Li Shu, C., Hoskins, R.A., Abad, J.P., de Pablos, B., Villasante, A., and de Jong, J. 2007. BAC clones generated from sheared DNA. Genomics 89, 291-299.

Ota, M., Katsuyama, Y., Kimura, A., Tsuchiya, K., Kondo, M., Naruse, T., Mizuki, N., Itoh, K., Sasazuki, T. and Inoko, H. 2001. A second susceptibility gene for developing rheumatoid arthritis in the human $\mathrm{MHC}$ is localized within a $70-\mathrm{kb}$ interval telomeric of the TNF genes in the HLA class III region. Genomics 71, 263-270.

Otting, N., Heijmans, C.M., Noort, R.C., de Groot, N.G., Doxiadis, G.G., van Rood, J.J., Watkins, D.I. and Bontrop, R.E. 2005. Unparalleled complexity of the MHC class I region in rhesus macaques. Proc. Natl. Acad. Sci. USA 102, 1626-1631.

Pamer, E. and Cresswell, P. 1998. Mechanisms of MHC class I--restricted antigen processing. Annu. Rev. Immunol. 16, 323-358.

Parham, P. and Ohta, T. 1996. Population biology of antigen presentation by MHC class I molecules. Science 272, 67.

Pascalis, H., Heraud, J.M., Fendel, R., Lavergne, A. and Kazanji, M. 2003. Molecular characterization of major histocompatibility complex class 1 (MHCI) from squirrel monkeys (Saimiri sciureus). Immunogenetics 55, 633-639.

Peelman, L.J., Chardon, P., Nunes, M., Renard, C., Geffrotin, C., Vaiman, M., Van Zeveren, A., Coppieters, W., van de Weghe, A., Bouquet,Y. 1995. The BAT1 gene in the MHC encodes an evolutionarily conserved putative nuclear RNA helicase of the DEAD family. Genomics 26, 210-218.

Pellet, P., Vaneensberghe, C., Debre, P., Sumyuen, M.H. and Theodorou, I. 1999. MIC genes in non-human primates. Eur. J. Immunogenet. 2, 239-241.

Pham-Dinh, D., Gaspera, B.D., De Rosbo, N.K. and Dautigny, A. 1995. Structure of the human myelin/oligodendrocyte glycoprotein gene and multiple alternative spliced isoforms. Genomics 29, 345-352.

Pham-Dinh, D., Jones, E.P., Pitiot, G., Della Gaspera, B., Daubas, P., Mallet, J., Le Paslier, D., Lindahl, C.F. and Dautigny, A. 1995. Physical mapping of the human and mouse MOG gene at the distal end of the MHC class $\mathrm{lb}$ region. Immunogenetics 42, 386-391. 
Pham-Dinh, D., Mattei, M. G., Nussbaum, J. L., Roussel, G., Pontarotti, P., Roeckel, N., Mather, I. H., Artzt, K., Lindahl, K. F. and Dautigny, A. 1993. Myelin/oligodendrocyte glycoprotein is a member of a subset of the immunoglobulin superfamily encoded within the major histocompatibility complex. Proc. Nat. Acad. Sci. USA 90, 7990-7994.

Ploegh, H. 1998. Viral strategies of immune evasion. Science 280, 248-253.

Rahman, A., Friedman, D.S. and Goldstein, L.S. 1998. Two kinesin light chain genes in mice. Identification and characterization of the encoded proteins. J. Biol. Chem. 273, 15395-15403.

Reveille, J.D. 2000. The changing spectrum of rheumatic disease in human immunodeficiency virus infection. Semin Arthritis Rheum. 30, 147-166.

Reymond, A., Meroni, G., Fantozzi, A., Merla, G., Cairo, S., Luzi, L., Riganelli, D., Zanaria, E., Messali, S., Cainarca, S., Guffanti, A., Minucci, S., Pelicci, P.G. and Ballabio, A. 2001. The tripartite motif family identifies cell compartments. EMBO J. 20, 2140-2151.

Rhodes, D.A. and Trowsdale, J. 1999. Genetics and molecular genetics of the MHC. Rev. Immunogenet. 1, 21-31.

Robinson, J. and Marsh, S.G. 2000. The IMGT/HLA sequence database. Rev. Immunogenet. 2, 518-531.

Rolleke, U., Flugge, G., Plehm, S., Schlumbohm, C., Armstrong, V.W., Dressel, R., Uchanska-Ziegler, B., Ziegler, A., Fuchs, E., Czeh, B. and Walter, L. 2006. Differential expression of major histocompatibility complex class I molecules in the brain of a New World monkey, the common marmoset (Callithrix jacchus). J. Neuroimmunol. 176, 39-50.

Roth, M.P., Malfroy, L., Offer, C., Sevin, J., Enault, G., Borot, N., Pontarotti, P. and Coppin, H. 1995. The human myelin oligodendrocyte glycoprotein (MOG) gene: complete nucleotide sequence and structural characterization. Genomics 28, 241-250.

Rowe, N. 1996. The pictorial guide to the living primates. East Hampton (NY): Pogonias Pr. 263 p.

Rylands, A.B., Coimbra-Filho, A.F., and Mittermeier, R.A. 1993. Systematics, Geographic Distribution, and Some Notes on the Conservation Status of the 
Callitrichidae. In: Marmosets and Tamarins: Systematics, Behaviour, and Ecology. Ed. A.B. Rylands. Oxford University Press: Oxford.

Saitou, N. and Nei, M. 1987. The neighbor-joining method: a new method for reconstructing phylogenetic trees. Mol. Biol. Evol. 4, 406-425.

Sawai, H., Kawamoto, Y., Takahata, N. and Satta, Y. 2004. Evolutionary relationships of major histocompatibility complex class I genes in simian primates. Genetics 166, 1897-1907.

Sawai, H., Kawamoto, Y., Takahata, N. and Satta, Y. 2004. Evolutionary relationships of major histocompatibility complex class I genes in simian primates. Genetics 166, 1897-1907.

Scholer, H.R., Ruppert, S., Suzuki, N., Chowdhury, K. and Gruss, P. 1990. New type of POU domain in germ line-specific protein Oct-4. Nature 344, 435-439.

Schrader, L. and Todt, D. 1993. Contact Call Parameters Covary with Social Context in Common Marmosets, Callithrix j. jacchus. Animal Behaviour 46, 1026-1028.

Seo, J.; Walter, L. and Günther E. 2001. Genomic analysis of MIC genes in rhesus macaques. Tissue Antigens 58, 159-206.

Seo, J.W.; Bontrop, R. Walter, L. and Günther E. 1999. Major histocompatibility complex linked MIC genes in rhesus macaques and other primates. Immunogenerics 50, 358-362.

Shiina, S., Tamiya, G., Oka, A. and Inoko, H. 2001. Published Only in Database. Homo sapiens 2,229,817bp genomic DNA of 6p21.3 HLA class I region.

Snell, G.D. 1948. Methods for the study of histocompatibility genes. J. Genet. 49, 87-108.

Snell, G.D. 1951. A fifth allele at the histocompatibility-2 locus of the mouse as determined by tumor transplantation. J. Natl. Cancer Inst. 11, 1299-1305.

Snoek, M., Groot, P.C., Spies, T., Campbell, R.D. and Demant, P. 1991. Fine mapping of the crossover-sites in the $\mathrm{C} 4-\mathrm{H}-2 \mathrm{D}$ region of $\mathrm{H}-2$ recombinant mouse strains. Immunogenetics 34, 409-412. 
Spies, T., Bresnahan, M. and Strominger, J.L. 1989. Human major histocompatibility complex contains a minimum of 19 genes between the complement cluster and HLA-B. Proc. Natl. Acad. Sci. USA 86, 8955-8958.

Stein, J. 2001. The magnocellular theory of developmental dyslexia. Dyslexia 7, 12-36.

Stephen, A.F., Madden, T.L., Schäffer, A.A., Zhang, J., Zhang, Z., Miller, W. and Lipman, D.J. 1997. Gapped BLAST and PSI-BLAST: a new generation of protein database search programs. Nucleic Acids Res. 25, 3389-3402.

Stevenson, M.F. and Poole, T.B. 1976. An Ethogram of the Common Marmoset (Callithrix jacchus jacchus): General Behavioural Repertoire. Animal Behaviour 24, 428-451.

Stevenson, M.F. and Rylands, A.B. 1988. The marmosets, genus Callithrix. In: Mittermeier RA, Rylands AB, Coimbra-Filho AF, da Fonseca GAB, editors. Ecology and behavior of neotropical primates, Volume 2. Washington DC: World Wildlife Fund. p 131-222.

Stewart, C. A., Horton, R., Allcock, R.J.N., Ashurst, J.L., Atrazhev, A.M., Coggill, P., Dunham, I., Forbes, S., Halls, K., Howson, J.M.M., Humphray, S.J., Hunt, S., Mungall, A.J., Osoegawa, K., Palmer, S., Roberts, A.N., Rogers, J., Sims, S., Wang, Y., Wilming L.G., Elliott, J.F., de Jong, P.J., Sawcer, S., Todd, J., Trowsdale, J. and Beck, S. 2004. Complete MHC Haplotype Sequencing for Common Disease Gene Mapping. Genome Research 14, 1176-1187.

Strausberg, R.L., Feingold, E.A., Grouse, L.H., Derge, J.G., Klausner, R.D., Collins, F.S., Wagner, L., Shenmen, C.M., Schuler, G.D., Altschul,S.F., Zeeberg,B., Buetow, K.H., Schaefer, C.F., Bhat, N.K., Hopkins, R.F., Jordan, H., Moore, T., Max, S.I., Wang, J., Hsieh, F., Diatchenko, L., Marusina, K., Farmer, A.A., Rubin, G.M., Hong, L., Stapleton, M., Soares, M.B., Bonaldo, M.F., Casavant, T.L., Scheetz, T.E., Brownstein, M.J., Usdin, T.B., Toshiyuki, S., Carninci, P., Prange, C., Raha, S.S., Loquellano, N.A., Peters, G.J., Abramson, R.D., Mullahy, S.J., Bosak, S.A., McEwan, P.J., McKernan, K.J., Malek, J.A., Gunaratne, P.H., Richards, S., Worley,K.C., Hale, S., Garcia, A.M., Gay, L.J., Hulyk, S.W., Villalon, D.K., Muzny, D.M., Sodergren, E.J., Lu, X., Gibbs, R.A., Fahey, J., Helton, E., Ketteman, M., Madan, A., Rodrigues, S., Sanchez, A., Whiting, M., Madan, A., Young, A.C., Shevchenko, Y., 
Bouffard,G.G., Blakesley, R.W., Touchman, J.W., Green, E.D., Dickson, M.C., Rodriguez, A.C., Grimwood, J., Schmutz, J., Myers, R.M., Butterfield, Y.S., Krzywinski, M.I., Skalska, U., Smailus,D.E., Schnerch, A., Schein, J.E., Jones, S.J. and Marra, M.A. CONSRTM Mammalian Gene Collection Program Team. 2002. Generation and initial analysis of more than 15,000 full-length human and mouse cDNA sequences. Proc. Natl. Acad. Sci. USA 99, 16899-16903.

Suarez, C.F., Cardenas, P.P., Llanos-Ballestas, E.J., Martinez, P., Obregon, M., Patarroyo, M.E. and Patarroyo, M.A. 2003. Alpha1 and alpha2 domains of Aotus MHC class I and Catarrhini MHC class la share similar characteristics. Tissue Antigens. 61, 362-373.

Sullivan, L.C., Hoare, H.L., McCluskey, J., Rossjohn, J. and Brooks, A.G. 2006. A structural perspective on MHC class $\mathrm{lb}$ molecules in adaptive immunity. Trends Immunol. 27, 413-420.

't Hart, B.A., van Meurs, M., Brok, H.P., Massacesi, L., Bauer, J., Boon, L., Bontrop, R.E. and Laman, J.D. 2000. A new primate model for multiple sclerosis in the common marmoset. Immunol Today 21, 290-297.

Takeda, J., Seino, S. and Bell, G. I. 1992. Human Oct3 gene family: cDNA sequences, alternative splicing, gene organization, chromosomal location, and expression at low levels in adult tissues. Nucleic Acids Res. 20, 46134620.

Tallmadge, R.L., Lear, T.L. and Antczak, D.F. 2005. Genomic characterization of MHC class I genes of the horse. Immunogenetics 57, 763-774.

Teraoka, Y., Naruse, T.K., Oka, A., Matsuzawa, Y., Shiina, T., lizuka, M., Iwashita, K., Ozawa, A. and Inoko, H. 2000. Genetic polymorphisms in the cell growth regulated gene, SC1 telomeric of the HLA-C gene and lack of association of psoriasis vulgaris. Tissue Antigens 55, 206-211.

The MHC sequencing consortium. Complete sequence and gene map of a human major histocompatibility complex. 1999. Nature 401, 921-923.

Thompson, J.D., Higgins, D.G. and Gibson, T.J. 1994. CLUSTAL W: improving the sensitivity of progressive multiple sequence alignment through sequence 
weighting, position-specific gap penalties and weight matrix choice. Nucleic Acids Res. 22, 4673-4680.

Timon, M., Elgar, G., Habu, S., Okumura, K. and Beverley, P.C. 1998. Molecular cloning of major histocompatibility complex class I cDNAs from the pufferfish Fugu rubripes. Immunogenetics 47, 170-173.

Trowsdale J. 1995. "Both man \& bird \& beast": comparative organization of MHC genes. Immunogenetics 41, 1-17.

Trowsdale, J. 1993. Genomic structure and function in the MHC. Trends Genet. 9, 117-122

Trtkova, K., Kupfermann, H., Grahovac, B., Mayer, W.E., O'hUigin, C., Tichy, H., Bontrop, R., and Klein, J. 1993. Mhc-DRB genes of platyrrhine primates. Immunogenetics 38, 210-222.

Trtkova, K., Mayer, W.E., O'Huigin, C. and Klein, J. 1995. Mhc-DRB genes and the origin of New World monkeys. Mol. Phylogenet. Evol. 4, 408-419.

Warren, R.D. and Crompton, R.H. 1997. Locomotor ecology of Lepilemur edwardsi and Avahi occidentalis. Am. J. Phys. Anthropology 104, 471-486.

Watkins, D.I., Chen, Z.W., Hughes, A.L., Evans, M.G., Tedder, T.F. and Letvin, N.L. 1990. Evolution of the MHC class I genes of a New World primate from ancestral homologues of human non-classical genes. Nature 346, 60-63.

Watkins, D.I., Garber, T.L., Chen, Z.W., Toukatly, G., Hughes, A.L. and Letvin, N.L. 1991. Unusually limited nucleotide sequence variation of the expressed major histocompatibility complex class I genes of a New World primate species (Saguinus oedipus). Immunogenetics 33, 79-89.

Watkins, D.I., Hodi, F.S. and Letvin, N.L. 1988. A primate species with limited major histocompatibility complex class I polymorphism. Proc. Natl. Acad. Sci. USA 85, 7714-7718.

Watkins, D.I., Kannagi, M., Stone, M.E. and Letvin NL. 1988. Major histocompatibility complex class I molecules of nonhuman primates. Eur. J. Immunol. 18, 1425-1433.

Watkins, D.I., Letvin, N.L., Hughes, A,L. and Tedder, T.F. 1990. Molecular cloning of cDNA that encode MHC class I molecules from a New World primate 
(Saguinus oedipus). Natural selection acts at positions that may affect peptide presentation to T cells. J. Immunol. 144, 1136-1143.

Watkins, D.I. 1995. The evolution of major histocompatibility class I genes in primates. Crit. Rev. Immunol. 15, 1-29.

Weiss, E.H., Kuon, W., Dorner, C., Lang, M. and Riethmuller, G. 1985. Organization, sequence and expression of the HLA-B27 gene: a molecular approach to analyze HLA and disease associations. Immunobiology 170, 367-380.

Weitkamp, L.R., Moss, A.J., Lewis, R.A., Hall, W.J., MacCluer, J.W., Schwartz, P.J., Locati, E.H., Tzivoni, D., Vincent, G.M. and Robinson, J.L. 1994. Analysis of HLA and disease susceptibility: chromosome 6 genes and sex influence long-QT phenotype. Am. J. Hum. Genet. 55, 1230-1241.

Wheeler, D.L., Church, D.M., Lash, A.E., Leipe, D.D., Madden, T.L., Pontius, J.U., Schuler, G.D., Schriml, L.M., Tatusova, T.A., Wagner, L. and Rapp, B.A. 2001. Database resources of the National Center for Biotechnology Information. Nucleic Acids Res. 29, 11-16.

Wong, Y.T., Del-Rio-Martin, J., Jaques, B., Shaw, J.A. and Talbot, D. 2005. Audit of diabetes in a renal transplant population. Transplant Proc. 37, 3283-3285.

Wroblewski, J.M., Kaminsky, S.G. and Nakamura, I. 1994. Bat-1 genes and the origin of multiple class I loci in the H-2D region. Immunogenetics 39, 276-280.

Wroblewski, J.M., Kaminsky, S.G., Milisauskas, V.K., Pittman, A.M., Chaplin, D.D., Spies, T. and Nakamura, I. 1990. The B144-H-2Db interval and the location of a mouse homologue of the human D6S81E locus. Immunogenetics 32, 200-204.

Wu, M.S., Tani, K., Sugiyama, H., Hibino, H., Izawa, K., Tanabe, T., Nakazaki, Y., Ishii, H., Ohashi, J., Hohjoh, H., Iseki, T., Tojo, A., Nakamura, Y., Tanioka, Y., Tokunaga, K. and Asano, S. 2000. MHC (major histocompatibility complex)DRB genes and polymorphisms in common marmoset. J. Mol. Evol. 51, 214222.

Yoshino, M., Xiao, H., Jones, E.P. and Fischer Lindahl, K. 1998. BAC/YAC contigs from the $\mathrm{H} 2-\mathrm{M}$ region of mouse Chr 17 define gene order as Znf173Tctex5-mog-D17Tu42-M3-M2. Immunogenetics 47, 371-380. 
Yuhki, N., Beck, T., Stephens, R.M., Nishigaki, Y., Newmann, K. and O'Brien, S.J. 2003. Comparative Genome Organization of Human, Murine, and Feline MHC Class II Region. Genome Research 13, 1169-1179.

Zhang, J., Zhang, L., Zhao, S. and Lee, E.Y. 1998. Identification and characterization of the human HCG $\vee$ gene product as a novel inhibitor of protein phosphatase-1 T. Biochemistry 37, 16728-16734. 


\title{
9 Annex
}

\section{1 - 55: The nucleotide sequences obtained from the MHC class-I region of} Callithrix jacchus. Sequences determined by the author with two exceptions (TS1 andTS2) stated in the text

\begin{abstract}
1. $204 \mathrm{C} 3$ ptarbac3
AAATGATCTTCAACGGGAATTCCTGTTCCACAGCGGACAGCGGTGTGAGCGCCCCAAAGCTGGAAATCAGAACCCTAAAG GCAGCTCGGCCCTCCCCAGCCCACAGCGCCGTTATTCCGTTTCTATATCGGTAAACACGCTTCATTTTTCCATAGCCCAGGG CGGGGTGACGGGTGATCCCAGTCTTCGCAGTGAACTGCGGGCAGTAGAATTCAAAAAGCTTGCGGGCAAAGCGGGGCGC GGTGGCCCACGCCTGTAATGCCAGTACGTTTGGGAGGTCGAGGCTGGCGGATCACCTGAGGTAGGGAGTTCGAGATCAG CCTGACCAACATGGAGAAACCCTGTCTCTACTAAAAATACAAAATTACCGGGCATGGGGCAACTGCCTGTAATCCCCCTATT CGTAGCTGGCGGCAGGAAAAGGGTTGCCCGAAGGCAA
\end{abstract}

\section{204C3 T729}

TCTACTTCTGAGTAGCTGGTATTATTGGCACCGCTGCCACCATGTCCGGCTAATTTTAAATTTTTTTGTAGAGACAGGGTCTT GTCATGTTGTCCAGGCTGGTCTCAAACTCCTGGCCTCAAGCAATTCTCTAGCCTTGGCCTCCCAAAGTGCTGGGATTACAA GTGTGAGCCATTGTGCCTAGCCAGTTTTTTGAATATTTTAAGCCTACTGTTGAGGCCTACTGCTCTGAAAAATAAAATTTCTT TAAAATGATTACTGCTCATTGACAATGTACATGGTTACCCAAGAGCCTCTAGCTCTGATGAAGATGTCCAAAGAGATGTATG TTTGTTTTCATGCCTGCTAACATAACATCCAGCCTGTACGCCCATGGTTTAAGGAGTAATTTAGACTTTCAAGTATTATTATTT AAGAAATACATTTTATAAGGTTGTATCTTCCATAGATAGTGAATCCTTTGATGGATCTGGGCAATGTAAACTGAAAGCCTCTG GAAGAGATTTTACCCAGATTCTAATTGCTCACATTTAGGACACATTTCACACACTGCCTGGGGCAATTGGGCGAGCCACCAC ATGGGCTTGTTAATCCCCAGAGCCCTTTTGGCCAGGGCACA

\section{510K19 ptarbac3}

AGTACTTCTAGGGAATTCATTACCACCAGCACCTGCATTACGAGAACTCCTGAAGGAAGCACTAAATTTGGAAAGGAAAGAT AGTTACCAGCCACTGTGAAAACACACTCAAGCAAGTACAGACTAGTGACACAATAAAGCAACCACTTAAGCAAGTCTGCAG CATATCATGGCGTGTAATATGAGAGACGCCGTACGTTTCTTTGTTACC

\section{217M17 T3}

AGTACTTATAGGgAATTATTACACCAGAACCTGCATTACGAGAACTCCTGAAGGAAGCACTAAATTTGGAAAGGAAAGATAG TTACCAGCCACTGTGAAAACACACTCAAGCAAGTACAGACTAGTGACACAATAAAGCAACCACTTAAGCAAGTCTGCAGCAT ATCATGGCGTGTAATATGAGAGACGCCGTACGTTTCTTTGTATA

\section{217M17T729}

CGTCTTCATAGTTGGAATTCCGAGTCCTGGCTGGAGCGCGATCTGGAATCTGACTCGCTTGAATTCTGACTCGGTGGATTC GGATCCGGGTGAGTAGGGAAATGCACCTCAGCCCCTCCCACGGGCGCCCACGGATTCTTGGATCCGAAAACGCTTCCTGC TGCTCAGTCACCCCAGGAAGGCAGCGCCCGCCTCTGGGCTGTTCTGATGGAAACAGGCTCCGCCGCCCGCAGGAAAACC CACAACTAAGGGGCCAGGAAAAAGCCCCTCAGGATCTCGCCACTTCAGTGAGGATCCTAATTTACACCCTCCATCAAAGAC TGCAGCCGCGTAACCTGGCAAAATCGGTTACGGTTGAGTAATAAATGGATGCCCTGCGTAAG

\section{347K7 ptarbac3}

TAAGTACTTCATAGGGAATTCCTTGACCTCAAGTGACCAGTCCGCCTTGGCCTCCCAAAGTACTGGGATTACAGGCAGGAA CCACCGCACCTGGCCACTAAACACGCTTTAACTTCTTTCCTCCTTCCGTCCCTCTTACTGTATCCTTCTAGGATTCCCCAAG TTCTGTTAAAAGCTTCTGGAACTGGAATCTAGCAAATGAATGTCCCATTTAATAGGCAGAGAAGGAGGAGGTGGGTGAATCA CAGAACCAAAGTGCATGTGGTGACAAGCTCCAGTTGTGCTCAGGGTGGGGTAGCTGGCAGGGCCCTGATGCATTCAGTCA TTGGTTCTGCAAATGTTTGCATAGTTCCACCAGCAGCATCTGCCCGGCAGAGAGCAGGACCAGGGCGAGGGTGAGGACAA GAGAGAGCACAGAGCCTGGATGGGGTAGTCAGCCACACAGGAACAACATGTGTAGGCTGAGTCATATCATGGCGTTGTAA TATGAG 


\section{7. $347 K 7$ ptarbac4}

GCTCCTCAATTACACGCCTGTATATGCTGCAGCCTACACATGTTGTTCCTGTGTGGCTGACTACCCCATCCAGGCTCTGTCT СTCTCTTGTCCTCACCCTCGCCCTGGTCCTGCTCTCTGCCGGGCAGATGCTGCTGGTGGAACTATGCAAACATTTGCAGAA CCAATGACTGAATGCATCAGGGCCCTGCCAGCTACCCCACCCTGAGCACAACTGGAGCTTGTCACCACATTGCACTTTGGT TCTGTGATTCACCCACCTCCTCCTTCTCTGCCTATTAAATGGGACATTCATTTGCTAGATTCCAGTTCCAGAAGCTTTTAACA GAACTTGGGGAATCCTAGAAGGATACGTTGAGGGAC

\section{347D1 ptarbac3}

GGTACTTCTAGGGAATTCCTGGACTCTCAGTGACTCACGTTCTGGCCTCCCAAAGTACTGGGATTACAGGCAGGAACACAC CGCACCTGGCCAGCTAAACACGCTTTAACTTCTTTCCTCCTTCCGTCCCTCATTACTGTATCCTTCTAGGATTCCCCAAGTTC TGTTAAAAGCTTCTGGAACTGGAATCTACGCAAATGAATGTCCCATTTAATAGGCAAAGAAGGAGGAGGTGGGTGAATCAC AGAACCAAAGTGCATTGGTGGTGACAAGCTCCAGTTGTGCTCAGGGTGGGGTAGCTGGCAGGGCCCTGATGCATTCAGAT CATTGGTTTCTGCAAATGTTTGCATAGTTCCACCAGCACCATCTGCCCGGCAGAGAGCAGGACCAGGGCGAGGGTGAGGA CAAGAGAGAGACAGAGCCTGGATGGGGTAGTCAGCCACACAGGAACAACATGTGTAGGCTGCAGCATATCATGGCGTGTA ATATGAGAGACGCCGTACGTTTCTTTGTATAGC

\section{347D1 ptarbac4}

TCTCTCTATTACACGCCTGATATGCTGCAGCCTACACATGTTGTTCCTGTGTGGCTGACTACCCCATCCAGAGCTCTGTCTC TCTCTTGTCCTCACCCTCGCCCTGGTCCTGCTCTCTGCCGGCGCAGCATGCTGCTGGTGGAACTATGCAAACATTTTGCAC GCAACCAATGACTGATTGCATCAGGGCCCTGCCAGCTACCCCACCCTGAGCACAACTGGAGCTTGTACCCAGCATTGCACT TTGGTTCTGATGATTCACCCACCTCCTCCTTCTCTGCCTATTAAATGGGACATTCATTTGCTTAGATTCCAGTTCCAGAAGCT TTTAACAGAACTTGGGGAATCCTAGCGCAAGGATACAGTAAGAGGGACGGAAGGAGGAAAGAATGTTAAAGCGTGTTTAGT GTGGCCAGGTGCCGCGTGGTTCCTGCCTGTAATCCCAGCTAGCTTTGGGAGGCCAAGGCGGACTGGCTCACTCGAGTGT CAGGAATTCCCTATGAAGATCCTTCTATAGTGTCACCTAAATGTAAT

\section{347D1 TCF19}

AGCTCTCTCCAGGCTGGGGCTGGGGCTGGCTTCCGCCCTATGCTGCCCTCCCTCGGGGCTCCACAGATCGGCCTCTTAG CACCTTGTCCTCTACCCCCAAGGCCACACTGATCCTGAACTCCATTGGCAGCCTCAGCAAGCTCCGGCCCCAGCCCCTCA CCTTCTCCCCTAGGGGGGGTAGACCAAAGATCTTGCCTGTTTCCGCCCCACCTGGGGAAGTGGGTATCATACCTTCTGCTC CACCCCCACGCAATCGGAGGAAATCTGCTCACCAAGTGTTAGCAGAACTGGATGATGAGAGTGAGCAGATTTCCTCGATGC GTGAGTGAGAGGTGGAGGACAAGATATAACTTCCGGTGGGGCGAAAGATATTTTGTGTATCTCCCCCTAGGGAGCAGAG AGAAGTGGAGGGTCTGGCACTCTGAACTCTCTGCCATAGTATGCGGATGGTATACACCTTCTGTGTAGACGACTACGTGCA GGCAGCAGTAGCAGCATGACATCCGACGCTAGCAGCGTGCGGTGACACGATGATCGACACTGACGAGACGACACGACAC GAGACGTACGTAGCTGATGACATAGCAGCGCTAGTCGTCGTAAGTCGAGCTAGACCATGACTGATCGAGTCAGTACATCAT GTCACGACTGCTACGACTGACATCGACTGACGTAGTGCGCGCATGTACGACTGCGACTGATCACATGCATGTACGCAGTA GCGAGCATGCATGCATGCTACGTCGACAGCTTGCATGGTAGCTACGATCAGCTGATAGCGATCGTACGATAGCTATAGATG CTCAGATCTAGTCGAGAGCATACGTACGTACTGCAGGCTAGAAGCTAGCTATGCGATGCATACGATGCATGAGATGCTAGC TCGCATGACGCGTACGACTACGGACTAGTCATGTAGATGACTCGAGTACGTACTGACTATCGATCGATATGATCAGATGCT AGTGTAGTATCATCAGATCAGCTATCACATTAGTAGATGTAGTCGACGTATCGATGATCATGTAGACTGATGACTGATAGAT CGATATACGATCGACGACTGACGATCGTCGGATACGTACGTAGCAGTCAGAGCCTCGCTGCTAGCAGTAGCGCACGCGAG AGATGCATGCTTCAACGTAGTACGATCAGTCATCGACTGCACTGCGTCGATGACGATCGATGCACGATCAGTAGCTGCTGT GAGCGATCGTATCAGCAGTACTAGTCAACGACGCAGACATCGTACGATCACAGTCGTACGTAGAGAGCAGTGATGTACGTA CTGACGTAGCTAGTAGTAGCATGATCGTACTGACTGATCGATGATGCTATGTGCATGACTGCATCTACGTCAGCACGTAGC AGTACGTCATGAGATGYCAGTCACTGACTGACGATGTCGACTGATGCATGACTGTAGTACTGATGAGCAGCTAGTGATCTG TACGCACATGCAGCAGTACGATGACGTACAGCTGCCTAGATCGATCGATCAGATCATAGACGCATATCTCAGAGTCATCAC GTACGATCGACTGACGAGATGATGATAGCAGCATGCAGAGTACGACGATACGATCACTAGTAGATGAGTCAGTGCATGCTA GCACGTGTAGCATGACGATCGATGACTGATCGTCAGTCACTGATGAGAGAATACGTGTCAGTCATGCATGTAGTTCAGTAT GAATCAGTTACCGTCATTGACATGTCAGTCAGCACTGCACTAGTGAGATCACGTAGTCAGCGATGATGTAGTGACTCAGCC TGCTAGCTATCGACGAGCTAGCAGTACGTTGCAGACTGTACGTACTACGTGTCAGCGTAGTCAGTCTACGTCAGTCATACT GCAGTCAAGCTACTCTGACTAGTCGATCGACTGGCTACGTCAGTACGTACGCATGCTGCATGCTATCGTGCAGCTAGTGTC GACTGACGTCAGCATGCAGTCAGTCAGATGATCATCGAGATGCATCGGTCATCAGATCAGCTAAGCTAGCAGATCGACTGA GACTGAGACGCAGCACTGATGACCTGACGATGCATGACTGACGTACGTCGATGCATGTCGATCGAGCTAGCACAGTCGCA 
GTAACCTATACGTACGTAGTCAACGATCCTGAATGACTATGCACTGCAGTCAGTCAGTCAGCTAGCTAACGTACTGAGTCAG ACGTACGTAGCTAGCTAGCGTACGTACTGAGTCACGATTCATGCTCATGATCATGCTAAGACGAGTACTAGCTCATTACGTG ATGCGATCGACGACGTACGACTACTGACGTACGACTGCTAGTCGTACAGCTG

\section{459020 ptarbac3}

AGTACTTCATAGGGAATTCTTCAGCCCATGATCCGCAAAACCTCCACATCCTCTCTCGGGATGTTCCTGCCCCTCGCAGCT CCAGCAGGAACCTGGGTTTCCCGGAAGACACAGACCCTTCGGGAGTCCTCCCACCTGGAGAGTTTCCCCAGTGACTTACA CСTCTGGACTCACTTCTCACTGTGGTTTTGAGAACTTTCTCTCTCССTCСTCССTAACCTCCСTAAGCTGTCATCAGATTAG GGCCCCACTCCСCTCATTGTAGCCATTCCTTGGGGGCCCCAGACCATTCCTCTCAATCCTGACTCTTGTAGCTCCTGGTTC ACTGGCTCGCTCTCCAGCAGTGCTGTCTCCTTCATCTTGATGACTTCAACATATGCAGATGTGGTGGGCTGAGTGATGGTC TCCACATAAGTTCTGTGTCAGTCCTTAGAACCTGTGAACAGGCTGAATTACATGGCAAAAGGGACACCGCTCATGTAATGAA GATTAGGGAACTTAAAATAGGGAGATTATCCTGGACTATTCGCATGGGCCCAGTCAAATGACATGAGCCATTAAAAGCAGA GAACCAACTCTGGCTGGAGTCAGAGATGTCGCGGAAGAGGGAGGGAAGAAGAGACA

\section{459020 ptarbac4}

TCATATTACACGCCATGTATATGCTGCAGCTCGTAGGGGCAGGTGCAGTGTAGGCAGCGGTGGAATCCACCATCTGTGTAG TTTTGCCAAGTAAGTGTGACAACACAAGGGAGAGGGGAGGGAGGGATGGAGATTATTTACCATAGAATCCAAATCCGGGG AGGAGGGAGTAGAGGACACCCAGGGCTGAGGGACAATGGGGAGATGGCAGGATCACTAGATTGGGAATCCTGGGGATTG GGTGTGTCRAAGGATTGCTGGAATTGATGTACTACAAGGTGGGCACCAAGTGTGAAATACAGGTACCTACAAAATGAATTAT TTGCTGATATCAAATTACAGCATATGGTAACATGACAGTATCTGTGTCCTTAAGAGCCTGTGGCCACCTTGCAAGGTGTGTA TGAGGGGGAAGATGGTCATGAGAGTGGAAGTGTGGATACTGTCCAGCTACTCTTCTAGAGGGGCTTGGGGTTCATTGGGC CCTGAAGGGAGCGCCTAGGATCGATTC

\section{3. $459020 \mathrm{T729}$}

TGTACTTCATAGTGGAATTCCATCAGTGCAGACTCAGGGACCACTGAGAACTACGTCACTGCAGCCGCGTAACCTGGCAAA ATCGGTTACGGTTGAGTAATAAATGGATGCCCTGCGTAAG

\section{4. $459020 \mathrm{~T} 729$}

ATTGCAGCTCGTAGGGGCAGGTGCAGTGTAGGCAGCGGTGGAATCCACCATCTGTGTAGTTTTGCCAAGTAAGTGTGACA ACACAAGGGAGAGGGGAGGGAGGGATGGAGATTATTTACCATAGAATCCAAATCCGGGGAGGAGGGAGTAGAGGACACC

\section{459020 TCF19}

CGAGCTCACTAGGAGGAGAGCTCAGGCTAGAGAGCTGGGGCTGGCTTCCGCTCCTATGCTGCCCTCACCACTACAGCGCT CCACAGCGGCCTCTTAACACCTTGTCCTCTACCCCCAAGGCCACACTGATCCTGAACTCCATTGGCAGCCTCAGCAAGCTC CGGCCCCAGCCCCTCACCTTCTCCCCTAGTTGGGGTAGACACAAAGATCTTGCCTGTATCCGCCCCACCTGGGGAAGTGG GTATCATACCTTCTGCT

\section{169G2 T729}

TGTACTTCTAGGGAATTCCAGGAACAAAGCAACCCAGTGAAGCTGAATCACAAAATCTGAGTATAAATGATTGAAATATTTGA TCAATCTCTAAATTACACAGATGCAGGATACACGCCTGCAGCCGCGTAACCTGGCAAAATCGGTTACGGTTGAGTAATAAAT GGATGCCCTGCGTAAG

\section{7. $459020 \mathrm{T729}$}

TGTACTTCATAGTGGAATTCCATCAGTGCAGACTCAGGGACCACTGAGAACTACGTCACTGCAGCCGCGTAACCTGGCAAA ATCGGTTACGGTTGAGTAATAAATGGATGCCCTGCGTAAG

\section{169G2 ptarbac4}

TGCTCTCATATTACACGCCATGAATGCTGCAGAGGCCGGATGGGGTCTGCTTTTTCTAGAACCCACCGTAGTCACTGCTGG GGTCCTCTTCATGGTGCACTCTAGTCCATGCCATGGGGATGATAAATAACCTTGGTGGATGGATTTAACAGTGCCAGGAAA CACTCTGCCTAGGGAGGAAGAAAATGGTTTGGTTTTAAGACTCCGTTCCTCTCTTAACCCTGGTGGTTTGTAATCTATCACT TCTGCCCAGTCCCGTTTCCCTGGCTCTGTGAGTCCTCTAGAGGTCACTGTTTTCTTCAGATCCTGACACTGACTACTAGACA TC 


\section{9. $123 \mathrm{H} 7 \mathrm{T729}$}

CGTACTTCATAGGGAATTCCTTTTGGAGGATGACAGTGGATTCATTGCCCTCGGGGGTTCAAACTAGTTATGAGTGAGGGA TTGGCCAGAAGATCGGGGCGCAGGCCAGCAGGAATGCTCTGTTAGGATAAGCAAGTTTGATAGGAAGAAGCTGCTCTTCT CCAAATTACAGAGAGGTGATGTGTTCGTATTGCACGTAGACTGTTTGTGTAACAAGACCTCCCCGCCCTGCCACCCCGACA CACAAACGAGCTGCCTAAAATATCCTTGCCTTGCAGATTGGAGGTTCTCCAAATATTTTGTGACCTGAGGATCCAGCTCAAG TGAGGTGCCATAGGATGCGTTCCTGAGTTTGCATTGTATGGAGACTTTTCTGGAATCTCTCAGTAGCAAGTCAGCTTCACAA CAAATTTTGCATTGAGTCCTAGACTGCTTGCAGTCTGAATTTGGACTACTTAGGTAGTGTGCTCAAAGTTGAACCTGGACAC AGCACAACTCAAGTTTGCATCAGACTGGGAAGCAAACTT

\section{169G2 TCF19}

TGGTCTAGGGGAGAGCTCCAGGCTGGGGCTGGGGCTGGCTTCCGCCCTATGCTGCCCTCCCTCGGGGCTCCACAGCGGC CTCTTAGCACCTTGTCCTCTACCCCCAAGGCCACACTGATCCTGAACTCCATTGGCAGCCTCAGCAAGCTCCGGCCCCAGC СССTCACCTTCTCCCCTAGGGGGGGTAGACCAAAGATCTTGCCTGTTTCCGCCCCACCTGGGGAAGTGGGTATCATACCTT CTGCTCCACCCCCACGCAATCGGAGGAAATCTGCTCACCAAGTGTTAGCAGAACTGGATGATGAGAGTGAGCAGATTTCCT CGATTGTCGGGGGGTGGAACAAAGGTTATGTACTATCCMGGGTGTGGCGAACAGGCAGATCTTGTGTTCTCCCCTAGGGG GGAGAAGGGGGAGGGGGCGGGCGATTTAAGCCACTGAATTTGGAAATTTGGCTGGGGTAAAAGAAGGTGTAACCGTGCA CAAGGAGGGCCTAAGGGGACCACCCCCCCGGCTTCCCCAACCGGGGGAGGATGCGCAGTGAGCTCGATAGCAGCATCAG CGACTAT

\section{1. $99 \mathrm{~L} 19$ TCF19}

GGCCCTAGGGGAGGAGTTCCAGGCTGGGCTGGGGCTGGCTTCCGCCCTTGCTGCCCTCCCTCGGCTCCACACGGCCTCT TAACACCTTGTCCTCTACCCCCAAGGCCACACTGATCCTGAACTCCATTGGCAGCCTCACAAGCTCCGGCCCCAGCCCCTC ACCTTCTCCCCTAGGGGGGGTAACCAAAATCTTGCCTGTTTCCGCCCCACCTGGGGAAGTGGRTATCATACCTTCTGCTCC CCCCAGCAATCGAGAAATCTGCTCACCAAGTGTTAGCAAAGCTGGATGATGAAAGTGACATTCCCCCGGGGGGGGGGGTA AAAAAAACAAACTCCCGCGGAAAAAAAAAATTTTTATTAAGGGGAGGGGGGGGGT

\section{TS1. 161C12-1}

AGCCTGCAGGTCATAATAGACCAATAGAAGGAGTGGGCATCGTGGTTCACACTTGTAATCCCTACATTTTGGGAAGCTGAG GTGGGAGGATCATTTCAGTTCAGGAGTTTGAGACCAGCCTGATAGTGAGACCTCATCTCTACCAAGTAAAAATAATTTTAAA AGGAAAAAGATCAACAGACAGGAAAGGAAGAAATATGTCTTTGTCACCAGCTTCATTGCACATGCAGAAAACACTAGGAAAT TCTGAAAAAGTCTCTGGAATTAATTATTGAATTTACAAATAGTTCAGAAAATATATGTGATAATCCACTTAGATGGACATGAAA AGATAGCAAACAATACTGGTCATCAAAGAAGTGAAAGTAAAAACCACAATCAGGAACCACCACACATCACCTGGAACAGGTA AAGTCAAAAAGACATATGATAATCTAAATACTGGCAAGAATATGGAACAAATACCAATGTCTGTATTGCTGGTTTGAATGCAA AAAATGTTGCAGCCAATTTGTAAAGTGGTTTAGCAATTTCTTATACAGCTGCCCATCCACTACCACAGGACCCAGGAATCCC ACATATATGTATTTATCCAAAAGAAATAAAAGTGTAAGGCCACACTTGTAAGCAGTTACTTGTAGTGGCTTCATTAATAACAC ACCCTAACTGGAGTGATCCACATGTCTATCAACTGGAGAACAGAAACCGATGAGTAAACTGGGATTCCAGCAATACTCAGC AGCTACTCAGCAACAAAAATCAATGAATGGCATCATCTCAAACATTGTTACGCTGCGTGAGAGATCAAACGAAGGACTACAT CACATATAATTGCACGTCCATGAAATTCTAGAAATTTCACTAATGCAGTGACAGAAAGCAGAGCAGTAGCTGAGTGAAGGGA AGGATTGGGGGTGGAAAGCTAGAATTAAATAGAAAGGGGCATAGAGAAAGTTTTTAGGGAAAAGAAACTGTTCTCTACAGT GTCACAATTCTGGAGTGACTGGGCAGTGGGAACTAAGGAGAAAGGAGGGAGGAGAGGGGCAGAGAGAAGGTCTGGGGAA GCAGGAGGTAAGGAGAAGGAGCAGGGAAAAGGACTCTAAAGCAGTGAAGGAGCCTAGCAGGGGGTTCTTTGCATGCAGA GTTTCTCTACTGACCAGTGTGGTAGTTACACGACTACAAATAATATCAATATGCACCAAAAAGCGCAGCTAAACCTGGTAAA TTTTATTATGCATAAATGCCCTAAGCCAAAAAAAAAAAAAAAAGGAGGCAGAGGGGAAGAAGGCAAATAAACACATTTTTAG ATCATCAAAACCATAAAATTTATTTCCTAGGGGTCCTGTACTACATGTAATTTTAAAGGAAGTTATTAACAACAGAAATCATGC ACACACACATATCACATACACACTCATTTTCTTAAGTTCCTGAAGGCATATGGCTCCTTTTCTAAAACAAAAACGATTACACT GTATTGTTGAGTTTGTATAGTATATTAGTGTAATATATACAACAATAACAGCACAATAGTAGGTTAAATGAAACTCCACTGTTA CAAGTGTCCTTTATTTTGCTGGATGTAGTTTAATATTACCTGAACTTCACTGTGAAAAGTCAAGGAATCGGGTTTCCATTCTT ACAACAATAAAACATGAGTGTAAAGAAATATAGCTAAAAGCCACTAGGAGTATTAAAACCATAACCTAAAAAATGTGTACTTG ACACATAAGAAACTAGCAAAGAAGGAACAGAAACATAAAGATATGAGACAAATTGAAAGCATATAGAAAATGGTAGACTTTTT CTTTTTTTTTTTTTTTTTGGCACCAAATTTCTTTATTTGAAGGAATGGTACAAAGAACTTAAGTGGATGTTTTGGTACAACTTAT AGAAAGGGTAAAGGGAACCCCAATATGCATGCAACTGTCTTGGTGACCAGGGAAGTCACCCCTTGGTTATGGGGAAATTAG CCTAAGTCTTAGCTTTTGCTATCACTGTCTTCCAGGGTGTGCTTGTCAAAGAGATATTCTTCCAAGCCAGATTCAGTTGCTCC 
CAACTTGAGCAAGTTGGTCACGTGGCCACCCAATTCTTTGATGGATTTCACCTGCTTATTCAGGTAATGTGTCTCAATGAAG TTTCACAAATGGGGGTCATTTTTGTCAGTGGCCAGTTTGTGCAGTTCCAGTAGTGACTGATTCACATCTTTTTCCAAATGTAA TGCACACTCCATCGCATTCAGCCCGCTCTTTCAGTCATCATGGTCTGGTTTCTTGACATCCTGAAGGAAGATGAGGCCACCT CGTTGGTTCTGCAGCTTCATCAGTTTCTCGGCATGTTCCCTCTCCTCATGAGATTGGTGAAGAAAGTACTTGGCAAAGTTCT TCAAAGCCACGTCATCACGGTCAAAGTAGTAAGACATGGACAGGTAAACATAGGAGGCGTAGAGCTCCGGGTTGATCTGG CGGTTGATGGCGGCCTCTGAATCCTGGTGGTAGTTCTGGCGCACCTGCGAAGGTGATGCGGTCGTCACCGCGGCTGCTAA GGAGAGGCGGCGGCGGCGGCGGTGGCTGCGCGGCGCTGGAGAGGCGGCGGGGCCTTGGAGAGGTCGGAGGGCGCGG TGTGGAGGTGAGGGAGGGCTGGCTGTGGGCGGCCGGCCGGGGTGGGGGACGAGCGCCGGGTTCCGTCCAAGCCCTGT TGAAGCAGGAAACCGCGGCGACTCTCGGCGGAGAACGTCTTGACTTTTTCACTTACATTGGTAGAATCCAACCATTTTAAGT GAAGAAATGACACGACCTGAGTCACATTAGCAGGATTGCTGAGCACTGTGGGGAGAACAGACATGGGCAGGAAGTGAGGG ACGGTGTTAGTGCCGCAATTCAGGAGTGACAGGGTGGCAGGGACTAAGGGGAGGGGAGGATGTGAGGGATGAGAGGGG CAAAGAGAAGGGATGGAGAAGCAGGAGGTGAGGAAAAGGGGCAGGGGAAAGAATTC

\section{TS2. 161C12-2}

GAATTCTTGTCCTGACTGTTCTGAGTAGTTCTGTAACTTGAGGCAATCACTTTTGTTATAAACCTCAGTGTTTGGTGGGGCG ACATAAAATTTTTAAATTAGGTGACCTCTAAAAGTTTATTGTAGTTTTGAAACATGCTAAAAATTGGTTTAGCTCAAACTGAGA GTGGGAACCCTGGGGGGATTGGACTCTTTCCTTCCTTGTTTTTGAGACTCTTTGCTCTTGGCTTGGCAGGTTGCTGTGTTTT TTGGTGGTCTGTCTATCAAGAAGGATGAAGAGGTGCTGAAGAAGAACTGCCCGCATATCGTCGTGGGGACTCCAGGCCGT ATCCTAGCCCTGGCTCGAAATAAGAGCCTCAACCTCAAACACATTAAACACTTTATTTTGGATGAATGCGATAAAATGCTTGA ACAGCTCGGTGAGTGGCAGTGCTGGGGCTTGGCTAATGCTGGGGAGTTGTTCTTTGGAGCCAAATGATGTTTATTTGAAAC AGGAGCACCTCAGTGTAAGGACGACTCTTATCTATCACCCATGACTGATGGCTCTGGGTTCCCTGGTTGGTTTTTATTCTTT TAAGCACAGTAAGGGGGTCATCTATCATCTTTCTATGATTTCTTTTTAACCTTTGAGAATAGGGGACTTTGATAATTTAGGCA TAAGTCGTCACCACCACTACCCTTTCCATTAAATATTTCTGTACTGGGAGTCATAGGGGATATTCTAAACTGAAGGAGAAGA CTGTCTACCCTTATGGCATCTCCAGCACAACATACACAGTCAGAATTTATACCTGAATCAGTTTCTAGACTCGGGTCTGAAAT TAATGTAGAGAGTGTTTGTAGGAGTTCATGTGGTGTGGTATTCTAGTGCTAAGTGGGGTTAATGAAGAAAGATTTTTCTGTC AGAATTGAATCTTGGTAAATGAGAGTGGGGATGGACATAGGCTCCAGAGAAAAGTTATTACAAATGATCTTTGGCAATTCAA TATGGCGAGCTATAAAGGTGGGCTCCAGGTAGAGATGTCTTATTTGTCCAACTGGATAGAAAAGTGATCCAGAGGGTCATA GATGAACTTTGATAGCTGGAATGTAATATGTGCTTGATATTTGTAGTCTGCTGAAGGCTGGCCTGGGCTTGGGCAGGAAAG AGTTGGGAGAATGGCCTGTAAAGCATGTTTTGAAGGCCTTATGCCTGAGAGTATCTGCACAGGGCTTTGTCCCCATTTTATA GTTGTGAACTTCGGGGTTTTTATCTTTCTTGCTTAAACAGTTGGAATATAATTCCAATTCTTGATTCCAAGTTCATTGCAGTCT TCACCTCACTGGGAGGTCTACCTTTAATCTTTACTATACCTGCTTATTTTCTCCTACAATTCTTTTCTTGTTCTCATAGACATG CGTCGGGATGTCCAGGAAATTTTTCGCATGACCCCCCATGAGAAGCAGGTCATGATGTTCAGTGCTACCTTGAGCAAAGAG ATCCGTCCAGTCTGCCGCAAGTTCATGCAAGATGTAAATACCCTTCTACCTTCTCTCCCTCCACTCCCCGCCCGCTGCCTC СTCССCTTCCTCGCCCTCTTCCTCCAGACTCCCTTGTCATTCAAGTGCCAAGAAGTCGGCTTATGCCCAGCTGGGAGTAAC GACTCCTTGAAGAGACATACAGAAGCAGAGACAGCTAGTGTTAGGGTCTGCGCGGGTGCCAGGGAAACTCCGGAAGACTT GGTCGGGTTAATGTGAGAGCGGGTAGTGTTCGACTTTTTCTCAAAAATCGCAACATTTTTGAACCTCTTCTCCTTTTGGGGG AGGGCAGGATTTTTCTGCCCTACCACCCACCCATCGTGTCTTACGTGCCCCCTACAGCCACGCATCCTCAAGGTGGCATCG AGCATACAGCTGGAGCCTTCTGCTCACCAAAACTCCTACTTCCCAGTGGCAGGAGAGCAAGAGAGGGACAGACAGATGGC AGGGCATGTCCAAAAGAAGAGCATCAGCACAGATGAATCCTCCCCTTCCCCACCTCCAGGGGTGGGGGCCTTTGGCACCT CAATCCCCGATACCCTACTCCTTCCCACCCACATCTCCTTGCACCCATCCGGAACCTCGGTTGATGTGAGCCGGCAACAGA GAAGCACCGTGGCGCGGCGAGGGAATGCGGACGGCACCCAGCGGTGGATGGCGGCAGCGGAGGCCGCGGGGAAACCT GACCAGGAAGCTGAGGACCAAACCAGCCTCTTTTTCCGTTCCCGGTTTTTTTCCTGAACCCAACGCGTGCCGTGCCCCGTT TCCCCCCATATGTGTTGGGGAGGGGTGTCCTGAATGGGGTGGTAGATTTTTTTTTTATTTTTTTTTTTTTTTCTTAAAAAAATT TTTTTTATTACTCAGAGGAGAGGGACATAGGAAAGGTAAAGTAGATGTAATCGGGTGAGTGTTAGGGTTTGGGGCTAGGTG GGTCCGATTGCATAAGCAGTGGAGTGTGTTCTTCCCCTCCCTGCAGTGTTCCTTCCTGTGGGATAACTCTTTATCTGTATTT GGGGCTAGAATGAAATTTCAAGGAGGCCATGGAACTTGTCTTTAAAAAGCCTGCCTTGGCTGAGCATGGTGGCTCACCTCT AATCCCAGTGCTTTGGGAGGCCAAGGTGGGAGGATTGCTTGAGCCCATTGAGACTAGCCTAGGCAATGTAGTGAGACCTT GTCTCTACCAAAAAGCTGGGTGTGGTGGCACATGCCTGTGGTCCCAGCTATCTGGGAGGCTAAGGCAGGAGGGTTTGCC TGAGCCCAGGATGTGGAGGCTGCAGTAAGCTGTAATTGTGCCACTGTACTCCAGCCTGGGTGATAGAGGCCCTGTATCAA AAAAAGAAAAGAAAACCTGCCTTGTTTTTTTTTTCCTATGGTACATGCATCATTTCCTATTTGGGCCGCGGGGTCTTCATGTT AACTATCTTTCCTAGGATAGTATAAAAAGTGTTAGGAAAATTGGGTTTTGGAGTTGTGCTAATTTC 


\section{261L9 HLA-B 29}

GGACATCGAGGGCTGCTTTTTTGCTTTTCTACCCAGGGCTGCTAGTGTTTTTYYTCCCTCCCTCATCCCCTCCTCCCACACCA ACTCCCAACCCACACACACCCCTGCACACTGCAGCACACAATCACGGTTCTCTCTTCAGGAGAGAATAGTCCTTGATGATG GGTCCAATTTCACAAACAAATGTAAGTCTAAATTACACTCTGCT

\section{271C5 T7.29}

GTGGGAACCTAATAACTTTTTATAGCATACATTACACGAAGTTGTATTAATGTGGTTCCGGATCAATTCTCATGTTTGACAGC TTATCATCGATAAGCTTTAATGCGGAGTTTATCACAGTTAAATTGCTAACGCAGTCAGGCACCGTGTATGAAATCTAAGCTAT CGC

\section{271C5 32A}

TCGGCCCCACCACCACCACCCCAGTCAGCAAATGCAGCACTTAATAAGGTTTCTCTCTTCAGAACATTTTTGACTATGGACC CATTTTACAACAACTGACGACTGAAATTAGACTGCTTTATAGATTCATGAGTTGGGATTGGAGCCAGCACCAAGATCACTGG AACCAGGGCAGGGAGAGAGGGCAGGAGAGCAGAGCAGAAGAGGAGCCCTAGAAGGAGGCCAGGAGCTGAATGGGTCTG AAAATGTCGTTCCAGACAGAGACCATCACCTTGCTGATCGTAGTCGTAGACAGATGCACGTGAGCTACTACGTACGTACGA TGCCATGTAAGTATACGATCTGAGACATGATCTATATCTACGCTAACTAGTACGTCAGTAAGTCGATCATACTGTACTAGCAT ACTGTAGTACAGAGATACGTACATCTTCATAACATAGACGACTCAGTAGTGTGCGTAGCAGCCACTTTAGCATCACTGCTAT GATGACTATACAGACTACGATCAGTACGACGATACGAACATGACTGCTGATCTCGTATGCATAATGATGCGCGCTGACTAC GTCTAC

\section{271C5 T7.29}

GTGGGAACCTAATAACTTTTTATAGCATACATTACACGAAGTTGTATTAATGTGGTTCCGGATCAATTCTCATGTTTGACAGC TTATCATCGATAAGCTTTAATGCGGYAGTTTATCACAGTTAAATTGCTAACGCAGTCAGGCACCGTGTATGAAATCTAAGCTA TCGC

\section{127E13 HLA-B29-}

TTTTGAAGTGAAGACTCACCCTCTGCTTTTCTGAGCAAGACGCTGCTTGTGTCCTCCCTCTGGCATCCCTCCTCTCACACCA ACCCCCCTGCACGCGCGTGCACACACACACACACACATTGCAGCACACAATCAGGTTTCTCTCTTCAGAAAAGAACAGTCC TTGATGATGGGTCCAATTTCACAAATAAATGTAAGTCTAAATTGGACTCTGCTTTATAGATTCATTATTTGGGGGTGGGGCCG ACACCAAGGTCACTAGGACCCGGGCACATAAACA

\section{127E13 HLB-29B}

CCCCGGTTACGAAAAGGTGGGGCTGGCTGTCTCCATCTCTGTCTCAACTTCATGGTGCACTGAGCTACAACCTCTTGCTTC CTTACTAAAAATAACAATCTGAATATAAATGTGCTTCCTCAAATATTTGCCATGAGAGGTTGATGAGTTAATTAAATCCCCACT TCCCCACCCC

\section{8. $282 L 12$ ptarbac3}

GAGTACTTATAGGGAATTCCTGCTCACAGCGGCGGGTGTGAGCGCCCCAAAGCTGGAAATCAGAACCCTAAAGGCAGCTC GGCCCTCCCCAGCCCACAGCGCCGTTATTCCGTTTCTATATCGGTAAACACGCTTCATTTTCCATAGCCCAGGGCGGGGTG ACGGGTGATCCCAGTCTTCGCAGTGAACTGCGGGCAGTAGAATTCAAAAAGCTTGCGGGCAAAGCCGGGCGCGGTGGCC CACGCCTGTAATGCCGTACGTTTCTTTGTATA

\section{9. $245 \mathrm{C} 6 \mathrm{~T} 729$}

AATTCGGGGGGACATTCATAGGGAATTCAGCCCCTCTGTCCACCCATTCTTCTCCCCACAGGATCGTCTGAGGAGTCGATT GGAAGCCCTGAGCATGGAGAGAGATGAGATTGAGGATGTAAAGTGTGGAGAAGATCAGAAGCTTCAAGTGCTGCTGGTAC AGGCCACGTCACTGTCTACCTTTTCCTTTGAAGGTTTTTCTTAACAGACTCTGGGGAAAACTGTTGGCTGGTATCTGTCGCC TGGCTGAAAAGAACTGGCACACTGTTCTCATTCACCT

\section{463N5 HLA-B}

AACACCAACACGACTCCGGGGCCTGCTGGTGCTTTTCTACGAGGGCTGCTAGTGTGTCCTCCCTCCCTCATCCCTCCTCCC ACACCAACTCCCAACCAACACACACCGCTGCACACTGCAGCACACAATCAGTCTTCTTTCTTCAGGAAAGAATAGTCCTTGA TGATGGGTCCAATTTCACAAACAAATGTCAGTCTAAATTACACTCTGCTTTATAGATTCATGAGTTGGGATTGGRGGCAGCA 
CCAAGATCACTAGAACCAGGGCAGGGAGAGAGGGCAGGACAGCAGAGCAGAAGAGGAGCCCTAGTAGCATGGCAGGAG GTGAATGGC

\section{463N5 ptarbac3}

TGACATCTAGAGGGCGCATTCGTTTCTCTACGCAGTTACCCAATAGTGGTGATCTACTTTTGGTTACTTAAGGATCTCCAAA CTGTTTTCCATAGTTGTTGTACTAGTTTGCATTCCCACCAGCGTGTAAAAGTGTTCCCTTTCACTGCATCCACGCCAACATCT ATTGTGCTTTGATATTTTGACTCTGGCCATTCTTGCAGGAGTGAGTTGCTATTGCACTGTGGTTTTGATTTGCATTTCCCCGC TCATTAGTGAGGTTGGCCATTTTTTCATATGTTTCTTGACCATTTGCATATTTCTTTCGAGAATTTTCTATTTATGCCCTCAGC ACATTTTTTGATGGGATTGTTTGTTCCTGTCCC

\section{436B4 T7.29}

CGCCGGCACCGGGGGCTGTTTTTTTGGCTTTTCTCCCCCAGGGCTGCTAGTGTTTTTCTCTCCCTCCCTCATCCCCTCCTC CCACACCAACTCCCAACCCACACACACCCCTGCACACTGAAGCACACAATCACGGTTCTCTCTTCAGGAGAGAATAGTCCT TGATGATGGGTCCAATTTCACAAACAAATGTAAGTCTAAATTAGACTCTGCTTTATAGATTCATGAGTTGGGATTGGACCCAA CATTCTGAAAATTTTTCTCAGACTGCTG

\section{325P21 T7.29}

GACTTCATAGTGGAATTCTGCACAATGGTCTCTCCTTTTTGAGGACTTCTCATTCTCTCCCATTCTGACATAGGCTGCTTACC TGGCTCATAGTCCAACTCAAAGCATAGTTTTTCTGTAAAGAAGAAATAAAACAGGATGATATTTTATTAGTCTTACAAAACCAT CAGACACTTAACAATGAGAAAACTGAGGCCCAGAGGAGGGAAGGGACAAGAAGAATGAAGCTGGAATCCTCTAGACCAGT TTCCAAGCTTGCATCAAATCACCTGGAGCACTTGCGAAAACACACCTGTTTCAATTCAGATGCTCTGGAGTGGGGA

\section{325P21 ptarbac3}

AAGACTTCTAGGGGAATTCTGGGTTTCTAACTCCTGTACAGAGACTGATTTTTGAGGGCCCGCCCCGCTCTCTGGGACAAT TAAGGGATGAAGTCTTTGAGGGAGTTGTGGGGAATTACAATCCAGGAATACTGATCACCTGTCCCTTTTGACCCCTGCACC ATATCATGG

\section{279K21 ptarbac3}

GGTCTTCTAGGGAATTTCTGGCCTCAGCGATCAGCCTGCCTCAGCCTCCCAAAGTGCTGGGATTACAGGTCTGAGCCACCA AGGCTCTTTCTTCCTGTCTCATGTAAGCACTTAAAGCTTCATGTTTCCCTCAGAGCAATGCCTGAGCTGCATTCCACAAACTT GGATATGTTGTTTTGTGACCATGCAATTCAAAATATTTTCTTACTTTCCTTGTGATTTTGTCTTTGGCCCTTAATTTACATAGAT AAATGTGGTTTCATTCCATACAGTTGATGTTTCCAAAATATCTTCTTTGTGTTAATTTCTAATTTAGTATTATCATAGAGAATAC ACTCTGGTGACTTTTACACTGTTGAATTAGTTGAGGCCCATTTTTCAGCTCAGCATATTGTCCTGGAATTGCACAGTGAATCT ACCGTATGTAATTGGTCACCTGTTTACCTGCTTTATATTTTCTGAGGGTTTGTGAGGTTTTCTCC

\section{224H20 T7.29}

CTCGAGAGAGGGAGTGCTTATTGTAGCGGCTTCCGAGATTTTAGCTGAGCTAAAACCCGACATTTGCTTCCCTATCTCATTT CTTGGCCTCATTGGTAATCTTTTAATGAGGAAGACATTTCCCCACGCGTGGAGCCGTGGCCCTTGACCTCTCACTCA

\section{7. $348 \mathrm{C} 21 \mathrm{T7} .29$}

CGTCTTCATAGGGAATTATCCCGCATGCTTGCTTGTCTATTTCTTCCACATGAAAAAGGCTTTCAGCCCACCTGGCTGAGCA GAAAGAATCTGAATGTTAGAGTTAGGTAGCCTGGGTTTGAATTCCATCTCGGGTACCACACTATGGCAAAATTCTTAGATTC TCCAAGCCTCAGTTGCCTTGTCTGCCAAATAGAGAACACATCCTCCCTCCTAAATTGTAAAAAGGATTAAAGTCATGTAAAGT GCATACTACAAATCCAGTCACAAAGTAGCTAGCTACTCACTAAGTGTTCAGCTCCTCCTTCCTCATTCAGATGGGAAGTGGC TTTAGATCAACAAAGTGGCAACCCAGTGGGCTTGAGCAGCACTGTGAACTTAGAATCCAAGAAAACTTGCAAAGAGCAGCT GGGAGGTAGTAGATCCTTGTGTTGCTTTTGGAAATTGTTCCATTTCTTTATTTCCCTATTGAATCTAGGCTCTAG

\section{171K8 T7.29}

TGACTTCATAGGGAATTCATCCCAGATGCTTGCTTGTCTATTTCTTCACATGAAAAAGGCTTTCAGCCCACCTGGCTGAGCA GAAAGAATCTGAATGTTAGAGTTAGGTAGCCTGGGTTTGAATTCCATCTCGGGTACCACACTATGGCAAAATTCTTAGATTC TCCAAGCCTCAGTTGCCTTGTCTGCCAAATAGAGAACACATCCTCCCTCCTAAATTGTAAAAAGGATTAAAGTCATGTAAAGT GCATACTACAAATCCAGTCACAAAGTAGCTAGCTACTCACTAAGTGTTCAGCTCCTCCTTCCTCATTCAGATGGGAAGTGGC 
TTTAGATCAACAAAGTGGCAACCCAGTGGGCTTGAGCAGCACTGTGAACTTAGAATCCAAGAAAACTTGCAAAGAGCAGCT GGGAGGTAGTAGATCCTTGTGTTGCTTTTGGAAATTGTTCACATTCTTTATTCACCTATTGAATCTAGACTCTAGCTAGAGAA AGAGCGGCAACCCTTGGCTTCAAATCCAGTGCTCTTCTCACACTCTTTAAGGTTGCTTTCCCGCTGCAGCCGCGTAACCTG GCAAAATCGGTTACGGGTTGAGTAATAAATGGATGCCCTGCGTAAGAAAACGCGCTCCCAAAT

\section{171K8 ptarbac2}

TTCTAACCGTAACCGATTTTGCCGGTTACGCGGCTGCAGCGGGAAAGCAACCTTAAAGAGTGTGAGAAGAGCACTGGATTT GAAGCCAAGGGTTGCCGCTCTTTCTCTAGCTAGAGTCTAGATTCAATAGGTGAATAAAGAATGTGAACAATTTCCAAAAGCA ACACAAGGATCTACTACCTCCCAGCTGCTCTTTGCAAGTTTTCTTGGATTCTAAGTTCACAGTGCTGCTCACAGCCCACTGG GTTGCCACTTTGTTGATCTAAAGCCACTTCCCATCTGAATGAGGAAGGAGGAGCTGAACACTTAGTGAGTAGCTAGCTACTT TGTGACTGGATTTGTAGTATGCACTTTACATGACTTTAATCCTTTTTACAATTTAGGAGGGAGGATGTGTTCTCTATTTGGCA GACAAGGCAACTGAGGCTTGRAGAATCTAAGAATTTTGCCATAGTGTGGTACCCGAAATGGAATTCAAACCCAGGCTACCT AACTCTAACATTCAGATTCTTTCTGCTCAGCCGGGTGGGCTGAAAGCCTTTTTGATGTGAAAAAAATAAAACCCGC

\section{171K8 Caja G}

CGCCTCGTGGCTGCCTCCCACTTGCGCTGGGTGATCTCGAGCCGCCAAGTCCGCGGCCGTCCCGGAGCGCAGGTCCTCG TTCAAGGCGATGTAGTCCTTGCCATCATAGGCGTGCTGGTTATACCCGCGGAGGAGGCGTCAGGCAGGCCCCAGGTCGCA TCCATACATCCTCTGGTAGGTGTGAGACCCTGGCCCCGCCCCCGAGGTCGGCCCCGCCCCAGCCCAACTTGCGGGGATT TTGGACCCAACTGAAAATGAAACCGGGTAAAGGCGCCTGGGGCTCTCCTGGATTGCGGGTTTGGGCGGGTGCCGCAGCC TCACGGTGAATCTTGGACCTGGAGACTCAGGGCGACCTGGGCCCT

\section{239N18 T7.29}

CTCTTCATAGGGAATTCATCCCAGCATGCTTGCTTGTCTATTTCTTCACATGAAAAAGGCTTTCAGCCCACCTGGCTGAGCA GAAAGAATCTGAATGTTAGAGTTAGGTAGCCTGGGTTTGAATTCCATCTCGGGTACCACACTATGGCAAAATTCTTAGATTC TCCAAGCCTCAGTTGCCTTGCTCTGCCAAATAGAGAACACATCCTCCCTCCTAAATTGTAAAAAGGATTAAAGTCATGTAAA GTGCATACTACAAATCCAGCCACACAAGTAGCTAGCTACTTAGTAAGTGTTCAGCTTCCTCCTTCCCTCATCC

\section{120D3 32b}

CCCCGGTTACGAAAAGGTGGGGCTGGCTGTCTCCATCTCTGTCTCAACTTCATGGTGCACTGAGCTACAACCTCTTGCTTC CTTACTAAAAATAACAATCTGAATATAAATGTGCTTCCTCAAATATTTGCCATGAGAGGTTGATGAGTTAATTAAATCCCCACT TCCCCACCCC

\section{120D3 HLAB 32B-}

TTTCAGCAGAAGTGGGGCTGGGTGCCTCCATCTCAGTCTCAACTTCATGGTGCACTGAGCTGCAACCTCTTACTTCCCTAC TGAAAATGAGAATCTGAAGATAAATGTGTTTTCTCAAATATTTGCCATGAGAAGTTGATGGGTTAATTAGTCAATTCCTAAAAT TTGAGAGAGGAAATAAAGACCTGAGAACCTTCCAGAATCTGCATGTTCACTGGCTGAGTCTGTTGCAGGTGGGGATGGA

\section{120D3 HLAB -29-}

TTGAATCGAGGCCCСTCTGCTTTTCTCAGCGGTGCTGCTTGTGTCCTCCСTCTGGCATCCCTCCTCTCACACCAACCCCCC TGCACGCGCGTGCACACACACACACACACATTGCAGCACACAATCAGGTTTCTCTCTTCAGAAAAGAACAGTCCTTGATGAT GGGTCCAATTTCACAAATAAATGTAAGTCTAAATTAACTCTGCTTTATAGATTTATAGTGGGGTGGGCAACA

\section{120D3-Caja-G}

TCAACACTGCTACGTCCCGGCCAGGCTGCGGGGAGCCCCGGTACTTCGAAGTCGGCTACGTGGACAACACGCAGTTCGT GCGGTTTGACAGCGACGCCGCGAGTCCGAGGATGGAGCCGCTGGCGCCGTGGGTGGAGCAGGAGGGCCGGAGTACTGG GACCCGGAGGCGCAGAAAGCCAAGGCCGCCGCACAGAATATCCGAGGGAACCGGCGGACCCTGCGCGGCCACTACAAC TAGAGCGGGCCGGTGAGTGACCTCGGCCCTGAGCGCAGGTCACGACGCCTCCCCATCCCCCACGGACAGCCCAGGTCCC CGTGAGTCTCCGGTTCCGAGATCATCCACCCCGAGGCTGCGAGACCCGCCCAGACCCTCGACCCGGGAGAGGCCCAGGC GCCTTTACCCGGTTTCATTTTCAGTTTAGGCCAAAATCCCCACGGATTGGTCCGGGCGGGGGCGGGGCTCGGTTGGTAGG GCTTACCTGGGGTCTGACCAGCGCCTCCTCTGCGGATATTACCAGTTAGCCTAAGACCGCAAGGACTACCTCTCTTTGAAC GAGGACTTGCGCTCCTGGACAGCCTCCTGCACAGCAGCTCAGATCACCCAGCCCAAGTGGGAGGCGGCCAGTGTGGCGG GAGCAGTGAAGAGCCTACCCTGGACGACACGTG 


\section{120D3 Caja-E-}

GCTTTCCTGCTCCGCCACACTGGCCGCCTCCCACTTGGGCTGGGTGATCTGAGCTGCTGTGCAGGAGGCTGTCCAGGAG CGCAAGTCCTCGTTCAAAGAGAGGTAGTCCTTGCGGTCTTAGGCTAACTGGTAATATCCGCAGAGGAGGCGCTGGTCAGA CCCCAGGTAAGCCCTACCAACCGAGCCCCGCCCCCGCCCGGACCAATCCGTGGGGATTTTGGCCTAAACTGAAAATGAAA CCGGGTAAAGGCGCCTGGGCCTCTCCCGGGTCGAGGGTCTGGGCGGGTCTCGCAGCCTCGGGGTGGATGATCTCGGAA CCGGAGACTCACGGGGACCTGGGCTGTCCGTGGGGGATGGGGAGGC

\section{120D3 MIC}

AAGTCCTTGGGTACATCTGGTCCTAATTAAGCTTGGAAACCTTGAGTTTGGTCCTCCCCATCCTGCTGCTAAGTCAATGTGA TCTTTGCCGAGTAGAAGCCCAGGGCCCAGCACCTCAGCCTCATGGTCAGAGATGGGGTGG

\section{120D3 Klex2-}

AACACACTGCTACGTCCCGGCCGGCTGCGGGGAGCCCGGTACTTCGAAGTCGGCTACGTGGACTTTCACGCAGTCGGCA ACCTT

\section{193P12 T7.29}

GTCTTCATATTGGAATTCTGGCAATCTCTCTCTTTTTTTTGCAATAAAAGCCTTATAAGAAACTTATTGAACGGCATAGGAATC AGTGCCGTTCAACTTGATTCAGAACGGTCAGTACATTATAATCTTGCCAAGGTACCTACTTATTAATTCCATGTCTTAATTTTA TGAAGTATTACAGGCTTTTAATACAGATAATTGGATATTTGCCACATATTTAAAAAAAATGTTATAAAATCTCTGTGTACTTGA TATCTAAGGTTTATTTTGGACTAAAACCCTATTACAGTATTCCTCAAAAGTGAAAATAAAATTTCCCTAACAAATAAAATAAAA AAGATACATAGATTGAATTGAAATAGGAAATTTCTAAATCCATGAACTGCAATTCAAAACTGGTTGTTTTCAGCCTTGCCCTA TTTCTCCTGATCTAGCTATCATCTTGACCTATAATGGTCCTGCTATGCCATAATATGAGGTTGCATCCTGAGAAGATATATTT GATTCTTATAAGACTCAGAGATACCGTAAGGGAAAATAGTGAATTCAAATAACCATGTGAAAGAACTATAAGCCCTCAAAATA TTGTGCTTAACCATAACGCCTCAATTGTACCAAAATTGTTGAACTATGTTTATTACTGACCTCCTTTAATTCAAAACCAACAAG ACCAACAAAGTTGATTCTTTGGCA

\subsection{G14 ptarbac2}

TTTTCGAGGCCTAATAACAAACTAGAGCGACAACATACGAAGCCCGTATTATGTGTGGCGGATAAAATCTCATGTTTGACAG CTTATCATCGATAAGCTTTAATGCGGTAGTTTATTACAGTTAAATTGCTAACGCAGTCCGGCACCGTGTATGAAATCTAACAA TGCGCTCATCGTCATCCTCGGCACCGTCACCCTGGATGCTGTAGGCATAGGCTTGGTCATGCCGGTACTGCCGGGCCTCT TGCGGGATATCTGCCATTCCGACAGCATGCCAGT

\section{1. $19116 \mathrm{~T} 729$}

GTCTTCATAGTGGAATTCCATTTCTACTCTGCCTCACCCAGTAAATTAATACAAATGCTTCAGAAGTCAGTTTAAGGGATTGT TCCATGACCCTACAGATCATAGTTTCTTCAGGTCAGGGTTTCTCAACAATGGCCCTATGGCATTTTGGACCAGGGAGTTGTT TGTTGTGGATAGCTGTCGTGTGCATTGTAGGATGTTTAATGGATGCCCTGCGTAAG

\section{174N1 T7-29}

AGACTTTCATATGGGATTCATCGCATATGCCTCAGTGTTTATGTGATCCAGAGCCTCACCACTGCCTATACAGACATGGGCA AACAGAGGAGAGTCTGGAAAATCATGTTGAACCAGCAAAAGTCATGCTTAGGGAATAGCCCTCTATGCAGTGGCACCCTCT TTTGCAGCCTCTTCTGGATTGGCTGAAGGAGGCGCATGA

\section{169G2 T729}

TGTACTTCATAGTGGAATTCCATCAGTGCAGACTCAGGGACCACTGAGAACTACGTCACTGCAGCCGCGTAACCTGGCAAA ATCGGTTACGGTTGAGTAATAAATGGATGCCCTGCGTAAG 


\section{Zusammenfassung}

Der Weissbüschelaffe Callithrix jacchus dient als Tiermodell zur Erforschung von Infektionen, Arzneimittelwirksamkeiten, der Parkinson-Krankheit sowie Organtransplantationen. Ferner wurde an ihm die Durchführbarkeit von Zell- und Gentherapie bei immunologischen und hämatologischen Störungen getestet. Dieser Neuweltaffe ist von besonderer Bedeutung wegen seiner Ähnlichkeit zum Menschen. Ziel dieser Arbeit war es die vier Klasse-I-Intervalle (Contig 1-4), einschließlich der flankierenden Framework-Gene, der MHC-Klasse-I-Region des Weissbüschelaffen Callithrix jacchus auf genomischer Ebene zu analysieren.

Hierzu wurde eine BAC-Bank (CHORI 259) von Callithrix jacchus mit Sonden von zwei MHC-Klasse-I-Genen und mehreren Framework-Genen gescreent. SouthernBlot- und Sequenzanalyse der MHC-Klasse-I-positiven BAC-Klone ermöglichte die Erstellung einer Karte über die Anordnung der MHC-Klasse-I-Gene im Weissbüschelaffen.

Die physikalische Kartierung der MHC-Klasse-I-Region von Callithrix jacchus liefert neue Grundlagen zur weiteren Analyse der MHC Evolution und zur Erforschung von verschiedenen Krankheiten, die mit MHC assoziert sind. Außerdem dient die Karte als Grundlage für die komplette Sequenzierung der MHC Region. 


\section{ACKNOWLEDGEMENTS}

The work described in this thesis has been carried out experimentally at the Deutsches Primatenzentrum, Abteilung Primatengenetik, under the supervision of PD Dr. Lutz Walter. The evaluation of the Callithrix sequences obtained was done at the MaxPlanck-Institut für experimentelle Medizin with guidance by Prof. Dr. Johannnes $\mathrm{H}$. Matthaei, to whome my special and sincere thanks and gratitude are due for his motivation, suggestions and discussions. Prof. Dr. Jürgen Wienands as my advisor has kindly contributed his counsel.

I like to thank Prof. Dr. Jürgen Wienands (Referent) and Prof. Dr. Ernst A. Wimmer (Korreferent) of the Universität Göttingen for their support. Sincere gratitude is felt towards my helpful advisors of the Institut für Genetik der Universität zu Köln, Prof. Dr. Thomas Wiehe and Prof. Dr. Jonathan Howard. Of the Göttingen Faculty of Biology, I wish to thank Prof. Dr. Hans Joachim Fritz, Prof Dr. Botho Bowien, Prof. Dr. Dieter Heineke and at Faculty of Medicine Prof. Dr. Nicolai Miosge. Internationally, Claire Amadou (France), Prof. Dr. Carlos Burgos (Colombia), Prof. Dr. Luis Fernando Cadavid (Colombia), Prof. Dr. Helena del Coral (Colombia) and Dr. Takashi Shiina (Japan), who kindly provided the sequences called TS1 and TS2. With gratitude I appreciate the invitation and support by Prof. Dr. Walter Stühmer, Director of the MPI für experimentelle Medizin.

I also thank all colleagues of our research group at the DPZ for the good and motivating working atmosphere, especially Mrs. Christiane Schwarz for her friendly help with lab work. I would like to say thanks a lot to, Anne (Anecita) Maicito (Conny), Jenicita (Jenny), Ulrikita (Ulrike) and Seray for their friendliness.

The Ph.D. scholarship granted by Deutscher Akademischer Austauschdienst (DAAD) is gratefully acknowledged.

Finally, I like to express my special thanks, to my family and to all the understanding and helpful people, of whom I mention Martin Mesa, Dr. Glenda Rodriguez, Dr. Maria Laura Ponce, Dr. Leslie Pineda, Maria Isabel Gomez, Omar Diaz, Dr. Jorge Alonso Leiva, Anne Averdam, Ute Rust, Svea Viola Dettmer, Dr. Andrea Gomez, Cyrilla Maelicke, Ralf Bosselmann and Sridhar Boppana, thanks for their help and friendliness. 


\section{Lebenslauf}

Natalia Regina Mesa Herrera

Geboren am

01.10.1970 in Medellín, Colombia

Eltern: Luis Hernan Mesa Santamaria

Maria Leonisa Herrera de Mesa

Familienstand: ledig

Staatsangehörigkeit: Kolumbianisch

Schulische Ausbildung:

1975-1981 Palermo de San Jose, Medellín, Colombia

1982-1989 Palermo de San Jose, Medellín, Colombia

Universitäre Ausbildung:

1990-1995 Bacteriologie und Klinischen Labor im Studiengang Mikrobilogie an Colegio Mayor de Antioquia, Medellín, Colombia

1996-1999

Anfertigung der Magister Scientiae-Arbeit an der

Universidad de Antioquia, Medellín, Colombia

Juni 1999

Magister Scientiae-Examen

Januar 04-August 07 Anfertigung und Anleitung der Dissertation am Deutschen

Primatenzentrum, Göttingen von Prof. PD. Lutz Walter (DPZ, Laborarbeit) und am Max-Plank-Institut für Experimentelle Medizin, Göttingen von Prof. Johannes $\mathrm{H}$. Matthaei (MPI, Auswertung und Textfassung). 